\title{
Design and flight testing actuator failure accommodation controllers on WVU YF-22 research UAVs
}

Yu Gu

West Virginia University

Follow this and additional works at: https://researchrepository.wvu.edu/etd

\section{Recommended Citation}

$\mathrm{Gu}, \mathrm{Yu}$, "Design and flight testing actuator failure accommodation controllers on WVU YF-22 research UAVs" (2004). Graduate Theses, Dissertations, and Problem Reports. 2630.

https://researchrepository.wvu.edu/etd/2630

This Dissertation is protected by copyright and/or related rights. It has been brought to you by the The Research Repository @ WVU with permission from the rights-holder(s). You are free to use this Dissertation in any way that is permitted by the copyright and related rights legislation that applies to your use. For other uses you must obtain permission from the rights-holder(s) directly, unless additional rights are indicated by a Creative Commons license in the record and/ or on the work itself. This Dissertation has been accepted for inclusion in WVU Graduate Theses, Dissertations, and Problem Reports collection by an authorized administrator of The Research Repository @ WVU.

For more information, please contact researchrepository@mail.wvu.edu. 


\title{
DESIGN AND FLIGHT TESTING ACTUATOR FAILURE ACCOMMODATION CONTROLLERS ON WVU YF-22 RESEARCH UAVS
}

\author{
by \\ $\mathrm{Yu} \mathrm{Gu}$
}

\author{
Dissertation submitted to the \\ College of Engineering and Mineral Resources \\ at West Virginia University \\ In partial fulfillment of the requirements \\ for the degree of \\ Doctor of Philosophy \\ in \\ Aerospace Engineering
}

Approved by

Marcello Napolitano, Ph.D., Chair

Larry Banta, Ph.D.

Bojan Cukic, Ph.D.

Gary J. Morris, Ph.D.

Brad Seanor, Ph.D.

Department of Mechanical and Aerospace Engineering

Morgantown, West Virginia

2004

Keywords: Fault Tolerance, Unmanned Aerial Vehicle, Neural Networks, Actuator Failure Accommodation 


\section{Abstract \\ DESIGN AND FLIGHT TESTING ACTUATOR FAILURE ACCOMMODATION CONTROLLERS ON WVU YF-22 RESEARCH UAVS by Yu Gu}

This dissertation describes the design, development, and flight testing of a Neural Network (NN) based Fault Tolerant Flight Control System (FTFCS) with the ability to accommodate for actuator failures. The goal of this research was to demonstrate the ability of a specific set of control laws to maintain aircraft handling qualities in the presence of failures in the actuator channels. In this study, two-failure scenarios have been investigated: aileron failure (locking of the right aileron at a trim position) and elevator failure (locking of the right elevator at a trim position). A fleet of WVU YF-22 research aircraft test-beds were manufactured and instrumented for developing and testing of flight control software. An on-board payload with a PC-104 format computer system, sensors, and custom made circuit boards were designed and developed for these aircraft test-beds. The fault tolerant flight control systems for this study were designed to recover the aircraft with damaged actuators. On-board real-time data acquisition and control software was developed to achieve the Actuator Failure Accommodation (AFA) flight demonstration.

For the purposes of this research, control laws were required to be adaptive to changing aircraft dynamics during a failure scenario. On-line learning NNs - with their non-linearity and learning abilities - were used in the design of the on-board aircraft control scheme. The on-line training reduced the criticality of an extensive on-line Parameter IDentification (PID) during the failure and gives an on-board flight controller the capability to adjust to maintain the best possible flight performance during an unexpected failure.

This document will outline and describe the design and building of the flight controller, aircraft test-beds, on-board payload systems, and software in detail. Flight test results will be presented and documented to demonstrate the performance of a $\mathrm{NN}$ based FTFCS under failure conditions. 


\section{Acknowledgements}

I would like first to thank my parents for their love, encouragement and support throughout my life.

I would like to thank my committee chairman and research advisor Dr. Marcello Napolitano. Your mentoring, guidance, and support throughout my graduate study has been never-ending. I am grateful for the vast and challenging research opportunities we have worked on together. You have always been there to guide and help me. Thank You.

I would like to thank my committee member Dr. Brad Seanor for your help and guidance with this research effort. Without your support, the success of this research project would not be possible.

I would like to acknowledge and thank my committee members Dr. Larry Banta, Dr. Bojan Cukic, and Dr. Gary Morris, for taking time from your busy schedules to review and contribute your thoughts to this research effort.

At this point, I would like to commend and thank all the flight testing members who helped develop the YF-22 research UAV. Many thanks to my pilot, Peter Cooke for your time and support in flying the YF-22s. I would like to acknowledge and thank the rest of the flight crew; Srikanth Gururajan and Larry Rowe for their hard work helping with the construction, flight testing, and instrumentation issues. I would like to acknowledge and thank Dr. Giampiero Campa and Sheng Wan for your help in estimating the linear mathematic model and design the linear controller used with this project. I would like to acknowledge and thank Larry Rowe for help building the printed circuit boards used on the YF-22 UAV payloads. All of your support and hard effort made the YF-22 AFA project possible. Thank You.

I would like to thank Chuck Coleman, David Estep, Lee Metheney, and Clifford Judy from the MAE department for their help with equipment and transportation issues.

Last but not the least, I would like to thank all of my research friends, past and present that have served their time down in the depths of the flight testing program. A special thanks goes out to all of you, who made graduate school a fun and enjoyable experience. 


\section{Table of Contents}

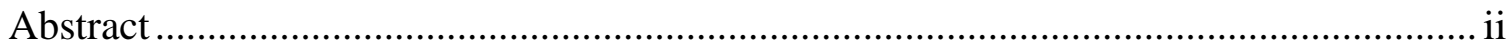

Acknowledgements ........................................................................................... ii

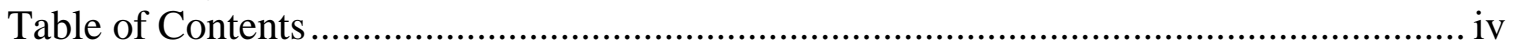

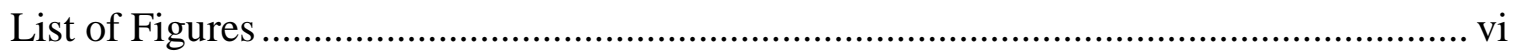

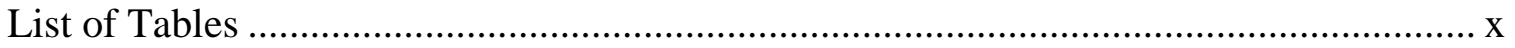

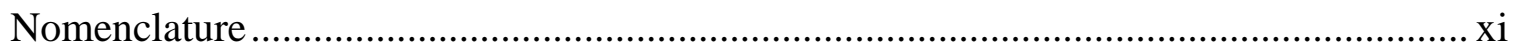

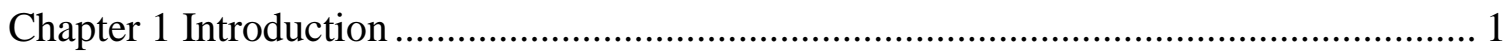

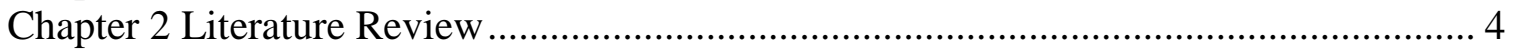

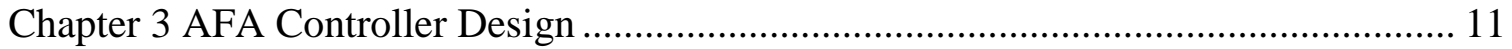

3.1 - Neural Network based Controller Design ..................................................... 12

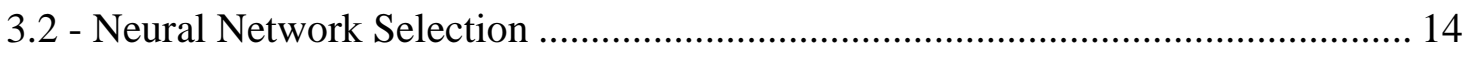

3.3 - Linear Mathematical Model .......................................................................... 23

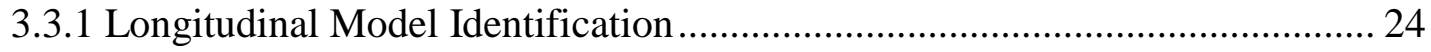

3.3.2 Lateral-Directional Model Identification ................................................ 28

3.3.3 Actuator Model Identification................................................................ 32

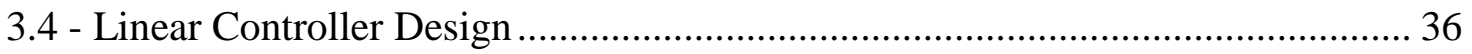

3.4.1 Longitudinal Control Parameter Design .................................................... 37

3.4.2 Lateral-Directional Control Parameter Design ........................................... 38

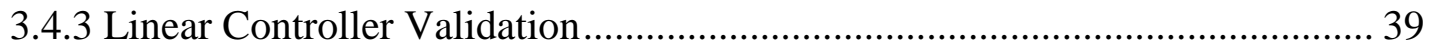

3.5 - Aileron Failure AFA Controller Design ................................................... 41

3.6 - Elevator Failure AFA Controller Design................................................... 49

Chapter 4 Test-bed Aircrafts \& On-board Payload ................................................... 59

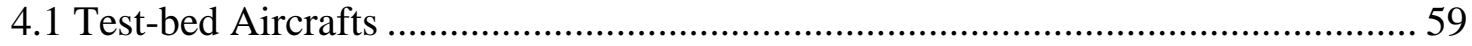

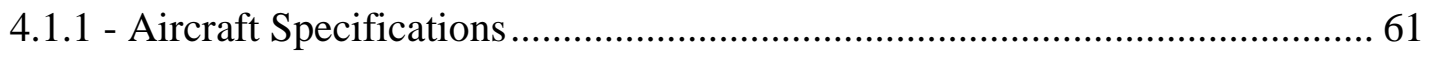

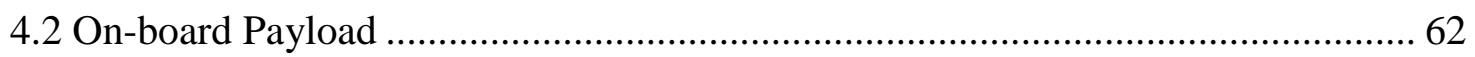

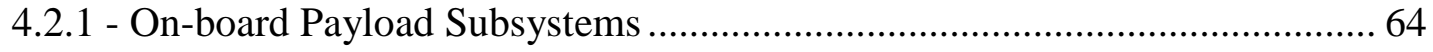

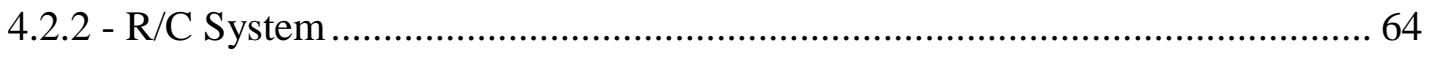

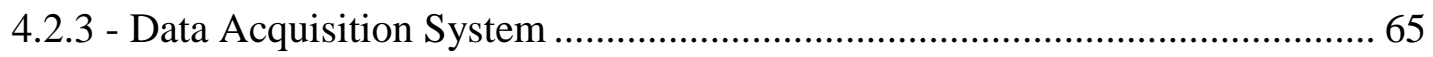

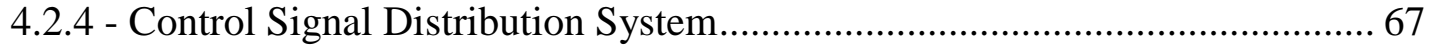

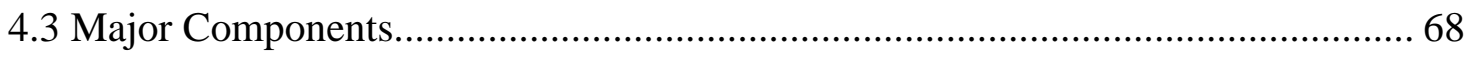

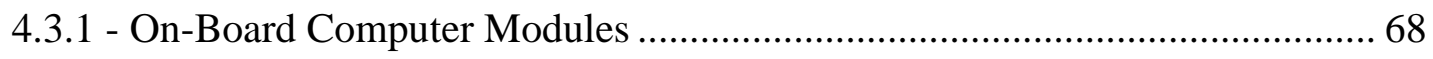

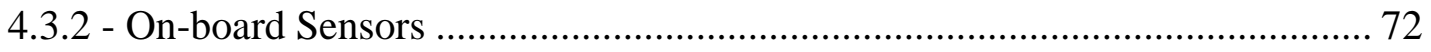

4.3.3 - Custom Designed Components: ......................................................... 77 


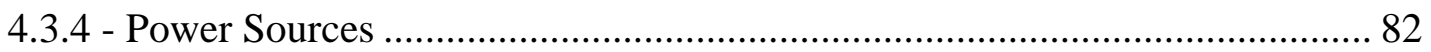

4.4 Hardware Mounting .................................................................................... 83

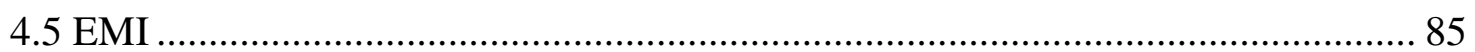

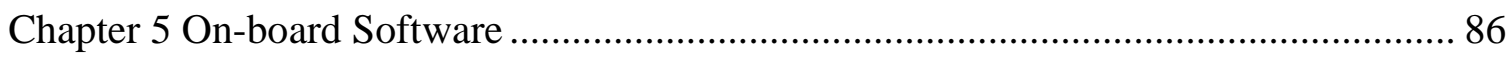

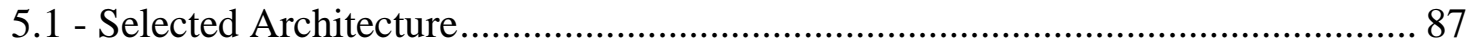

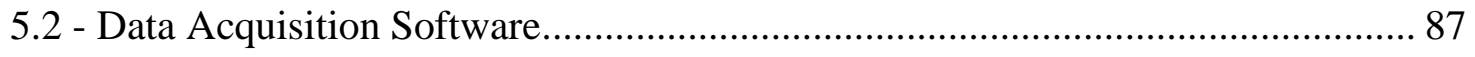

5.3 - AFA Flight Control Software .......................................................................... 94

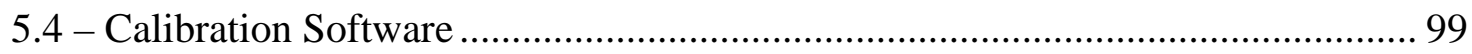

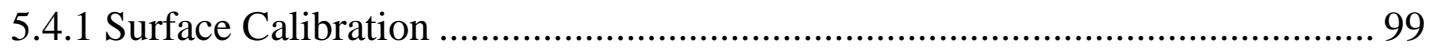

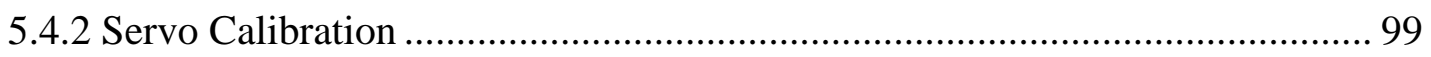

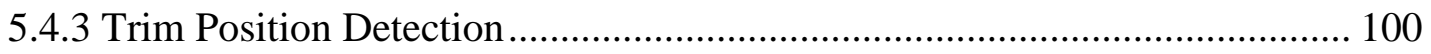

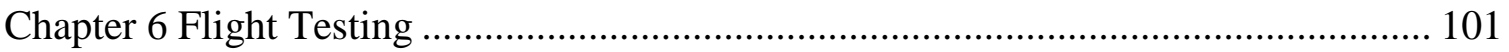

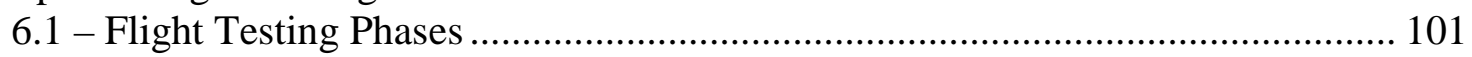

6.2 - Flight Testing Procedures ........................................................................... 108

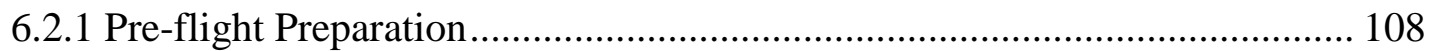

6.2.2 In-flight Procedures ................................................................................ 108

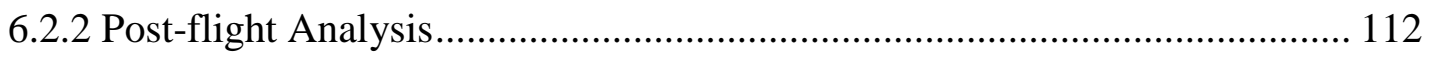

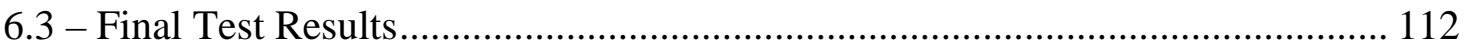

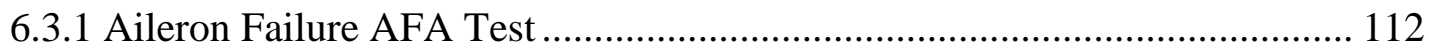

6.3.2 Elevator Failure AFA Test...................................................................... 122

Chapter 7 Conclusions and Recommendations......................................................... 137

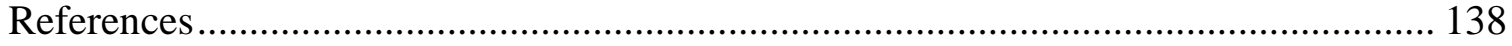




\section{List of Figures}

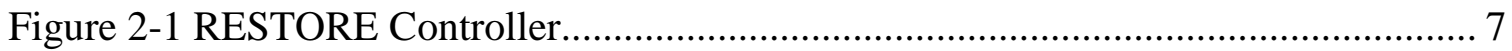

Figure 2-2 General Block Diagram of the IFCS GEN I Controller................................ 8

Figure 2-3 General Block Diagram of the IFCS GEN II Controller............................... 9

Figure 3-1 Architecture of the NN Controller ........................................................... 13

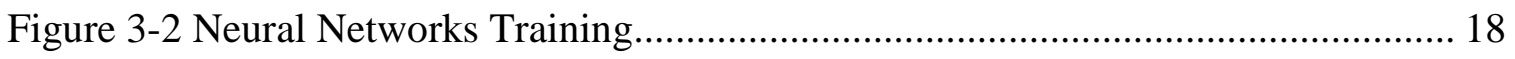

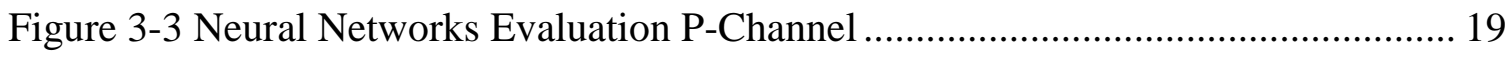

Figure 3-4 Neural Networks Evaluation R-Channel................................................... 19

Figure 3-5 Neural Networks Evaluation P-Channel (w/ failure) ................................... 20

Figure 3-6 Neural Networks Evaluation R-Channel (w/ failure).................................. 21

Figure 3-7 Neural Networks Estimation of Actuator Failure P-Channel (w/ on-line

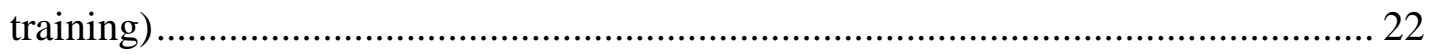

Figure 3-8 Neural Networks Estimation of Actuator Failure R_Channel (w/ On-line

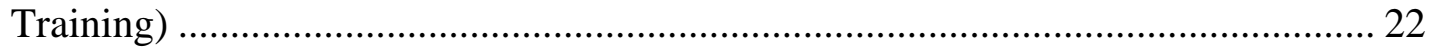

Figure 3-9 Data for Longitudinal Model Identification ........................................... 25

Figure 3-10 Data for Longitudinal Model Validation.............................................. 26

Figure 3-11 Model Validation - Measured and Simulated Pitch Rate ........................... 27

Figure 3-12 Model Validation - Measured and Simulated Angle-of-attack ................... 27

Figure 3-13 Data for Lateral-Directional Model Identification ................................... 28

Figure 3-14 Data for Lateral-Directional Model Validation......................................... 29

Figure 3-15 Model Validation - Measured and Simulated Sideslip Angle ..................... 30

Figure 3-16 Model Validation - Measured and Simulated Roll Rate ........................... 31

Figure 3-17 Model Validation - Measured and Simulated Yaw Rate ............................ 31

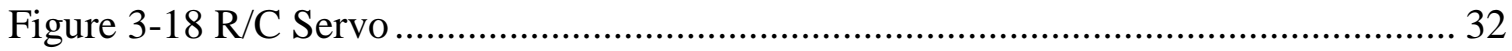

Figure 3-19 Data from Actuator Identification ................................................... 33

Figure 3-20 Measured and Simulated Actuator Step-response..................................... 34

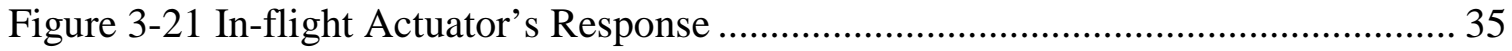

Figure 3-22 Model Validation - Measured and Simulated Actuator In-flight Response. 36

Figure 3-23 Root-locus (Longitudinal Dynamics)..................................................... 37

Figure 3-24 Linear Controller Performance after Elevator Maneuver ........................... 40

Figure 3-25 Linear Controller Performance after Aileron Maneuver.............................. 40 
Figure 3-26 Linear Controller Performance after Rudder Maneuver ............................. 41

Figure 3-27 Aileron Failure Response - P channel ............................................... 42

Figure 3-28 Aileron Failure Response- Coupling.................................................... 43

Figure 3-29 Linear Controller with/without Failure - Left Aileron .............................. 44

Figure 3-30 Linear Controller with/without Failure - P channel ................................. 45

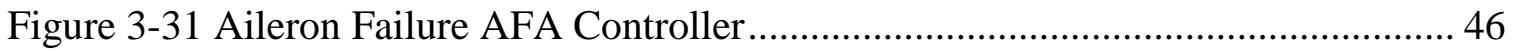

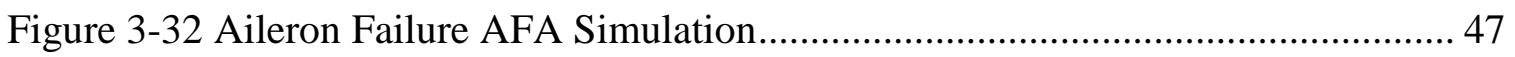

Figure 3-33 Aileron Failure AFA Controller Simulation - Gain Updating..................... 47

Figure 3-34 Performance Comparison of Three Conditions - P Channel ....................... 48

Figure 3-35 Elevator Failure Response - Q Channel .................................................. 49

Figure 3-36 Elevator Failure Response - Coupling ................................................. 50

Figure 3-37 Linear Controller with/without Failure - Left Elevator ............................. 51

Figure 3-38 Linear Controller with/without Failure - Q Channel ................................ 52

Figure 3-39 Linear Controller with/without Failure - P Channel................................. 52

Figure 3-40 Elevator Failure AFA Controller Design ............................................ 53

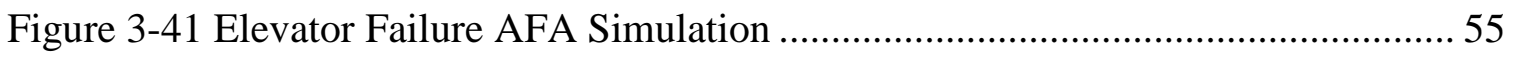

Figure 3-42 Elevator Failure AFA Simulation - Gain Updating ................................. 56

Figure 3-43 Elevator Failure AFA Simulation - Q Channel ........................................ 57

Figure 3-44 Elevator Failure AFA Simulation - P Channel...................................... 57

Figure 4-1 WVU YF-22 Research Model Aircraft (Version 1)..................................... 59

Figure 4-2 WVU YF-22 Research Model Aircraft (Version 2)..................................... 60

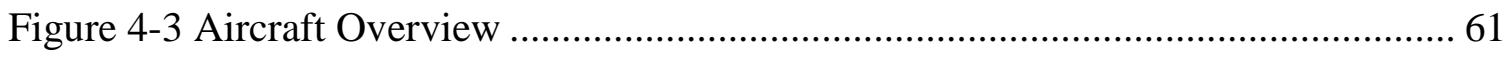

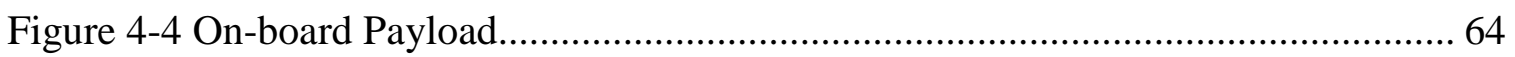

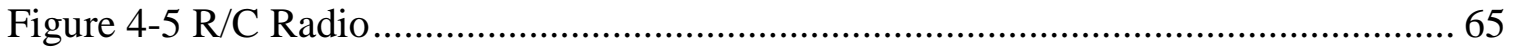

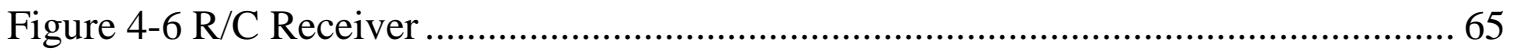

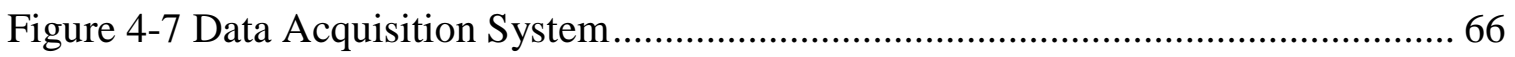

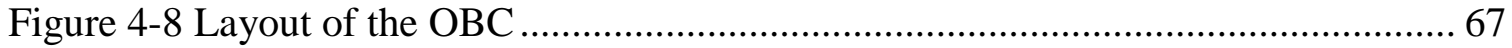

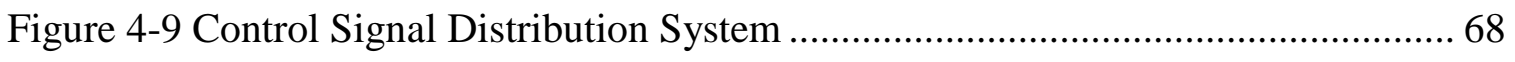

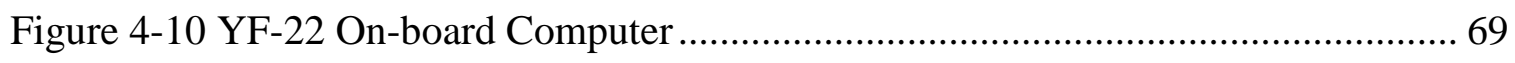

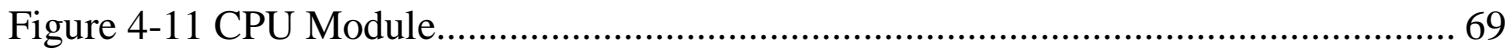

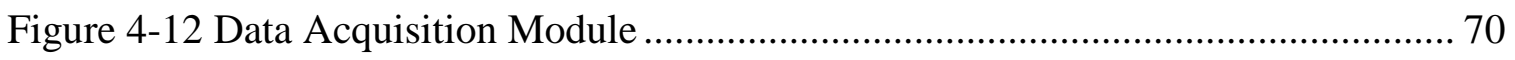




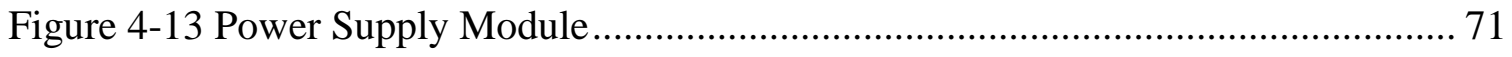

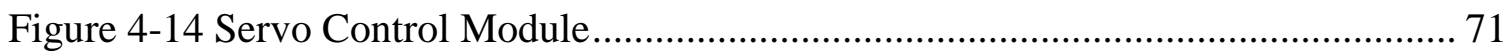

Figure 4-15 Compact Flash Card and Reader.......................................................... 72

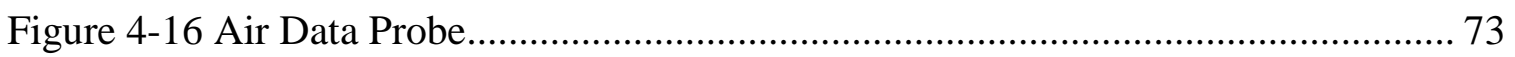

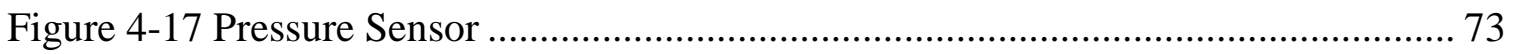

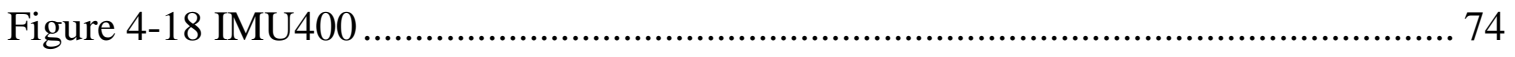

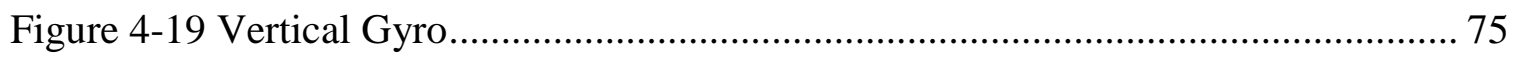

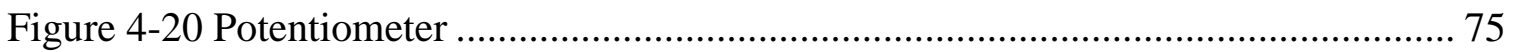

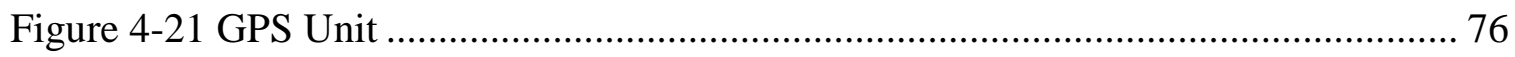

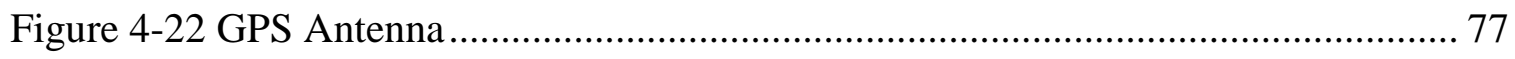

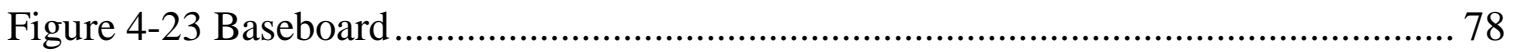

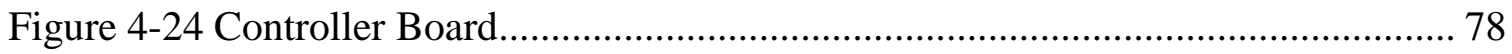

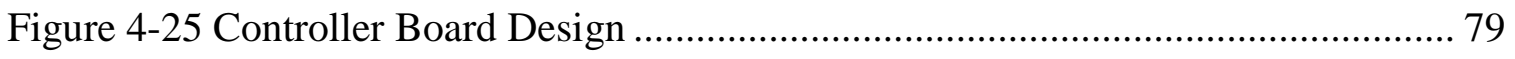

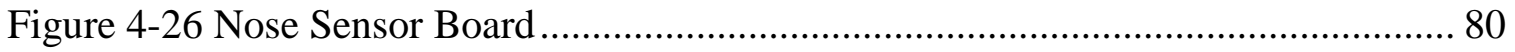

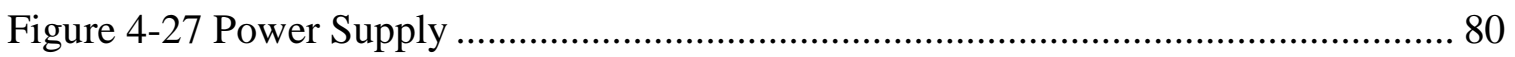

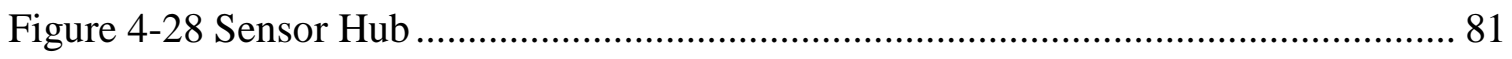

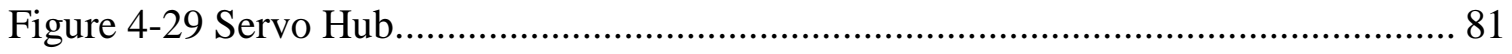

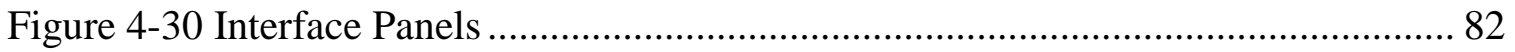

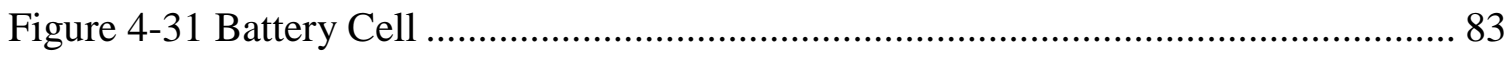

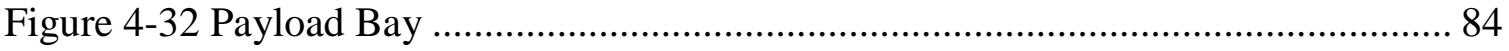

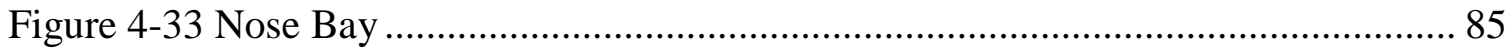

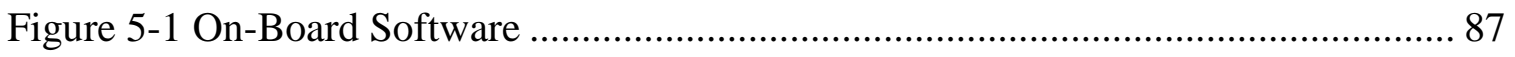

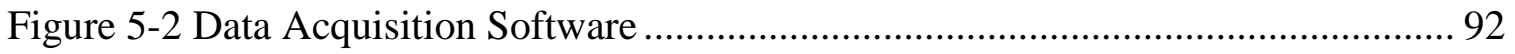

Figure 5-3 Simulink Diagram for On-Board AFA Software ........................................ 95

Figure 5-4 Simulink Diagram for Aileron AFA Controller.......................................... 97

Figure 5-5 Simulink Diagram for Elevator AFA Controller ...................................... 97

Figure 6-1 Flight Data Following Aileron-Rudder Doublet combination ...................... 103

Figure 6-2 Flight Data Following Elevator Doublet ................................................ 104

Figure 6-3 Aileron Deflection with Failure ........................................................ 105

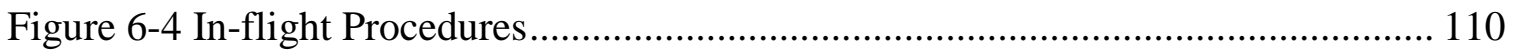

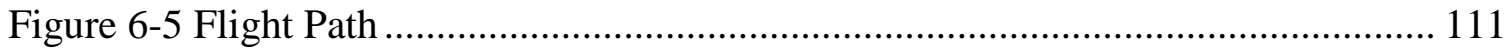




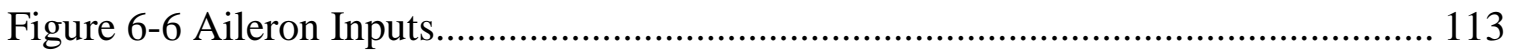

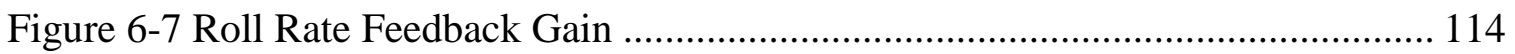

Figure 6-8 NN Approximations ............................................................................ 115

Figure 6-9 Roll Rate Response (First Failure Activation) ........................................... 116

Figure 6-10 Roll Rate Response (Last Failure Activation) ............................................. 116

Figure 6-11 Aileron Control Inputs ........................................................................ 117

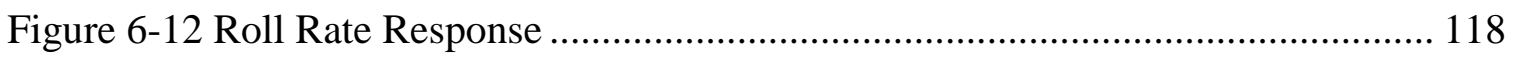

Figure 6-13 Statistical Analysis - Aileron Failure ………………………………...... 120

Figure 6-14 Performance Comparison - Left Aileron ...................................................... 121

Figure 6-15 Performance Comparison - Roll Rate................................................... 121

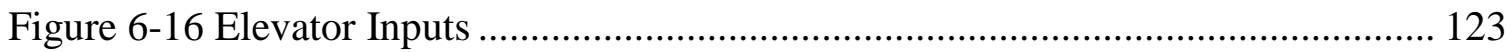

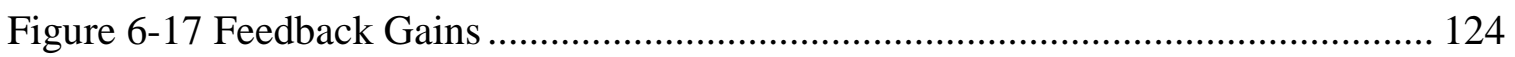

Figure 6-18 Pitch Rate Estimations ......................................................................... 125

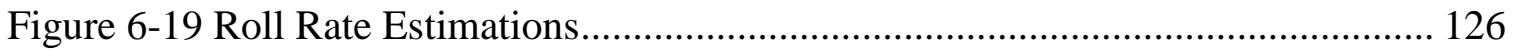

Figure 6-20 Aircraft Response (First Failure Activation).............................................. 127

Figure 6-21 Aircraft Response (Last Failure Activation) .............................................. 128

Figure 6-22 Left Elevator Deflections ...................................................................... 129

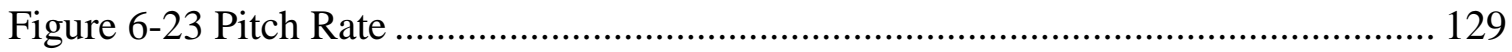

Figure 6-24 Aileron Deflections ............................................................................. 130

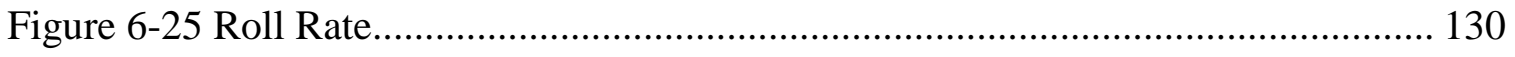

Figure 6-26 Elevator Failure Statistical Analysis -Longitudinal .................................. 132

Figure 6-27 Elevator Failure Statistical Analysis -Lateral ............................................. 133

Figure 6-28 Performance Comparison - Left Elevator................................................ 134

Figure 6-29 Performance Comparison - Pitch Rate ……………………………….... 134

Figure 6-30 Performance Comparison - Left Aileron ..................................................... 135

Figure 6-31 Performance Comparison - Roll Rate....................................................... 135 


\section{List of Tables}

Table 3-1 Inputs of the Lateral-Directional Neural Networks ...................................... 17

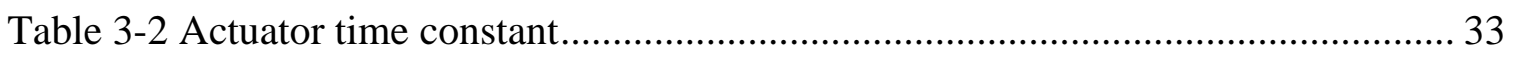

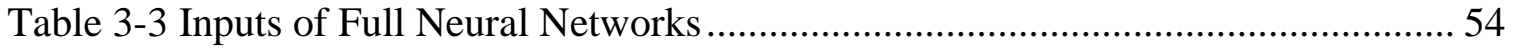

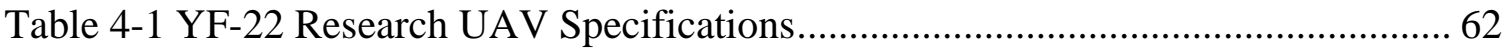

Table 4-2 On-board Payload Power consumption ......................................................... 83

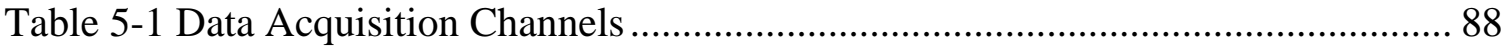

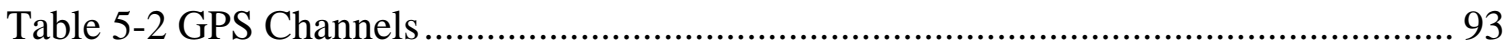

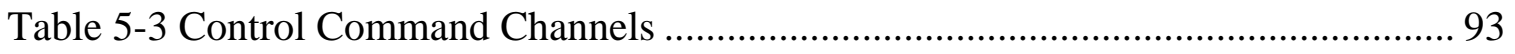

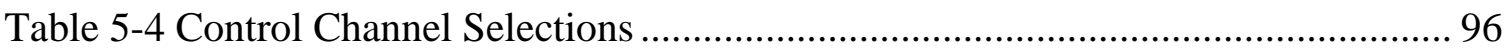

Table 6-1 Statistical Analysis - Aileron Failure ..................................................... 119

Table 6-2 Performance Comparison - Three configurations ..................................... 122

Table 6-3 Statistical Analysis - Elevator Failure ................................................... 131

Table 6-4 Performance Comparison - Three configurations ....................................... 136 


\section{Nomenclature}

\section{English}

a Acceleration, $\mathrm{m} / \mathrm{sec}^{2}$

a Node in input layer

b Node in hidden layer

b Wing span, $\mathrm{ft}$

B Node in hidden layer

c Node in output layer

$c \quad$ Mean aerodynamic chord, $\mathrm{ft}$

C Node in output layer

d Target Neural Network output

e Neural Network training error

g Acceleration due to gravity, $\mathrm{m} / \mathrm{sec}^{2}$

$\mathrm{J} \quad$ Error cost function

I Moment of inertia, slug- $\mathrm{ft}^{2}$

$\mathrm{k} \quad$ Discrete time index

m Aircraft mass, slugs

$\mathrm{P} \quad$ Aircraft angular velocity, $\mathrm{x}$ body axis (roll rate), $\mathrm{deg} / \mathrm{sec}$

Q Aircraft angular velocity, y body axis (pitch rate), deg/sec

$\mathrm{R} \quad$ Aircraft angular velocity, $\mathrm{z}$ body axis (yaw rate), deg/sec

$\mathrm{R} \quad$ Auto or cross correlation function

$\mathrm{S} \quad$ Wing planform area, $\mathrm{ft}^{2}$

t Time, sec

$\mathrm{u} \quad$ Velocity along body fixed $\mathrm{x}$-axis, $\mathrm{m} / \mathrm{sec}$

$\mathrm{U} \quad$ Upper bound of modified sigmoid activation function

V Velocity along body fixed y-axis, $\mathrm{m} / \mathrm{sec}$

$\mathrm{V}$ Interconnection weight vector between input and hidden layer nodes

W Velocity along body fixed $\mathrm{z}$-axis, $\mathrm{m} / \mathrm{sec}$

W Interconnection weight vector between hidden and output layer nodes

y Neural Network output 


\section{Greek}

$\alpha \quad$ Angle of attack, deg

$\beta \quad$ Angle of sideslip, deg

$\delta \quad$ Control surface deflection, deg

$\delta \quad$ Neural Network output and hidden layer error term

$\Delta \quad$ Error update term

$\eta \quad$ Neural Network learning rate

$\theta \quad$ Pitch Euler angle, deg

$\Theta \quad$ Neural Network hidden layer neuron threshold

$\Gamma \quad$ Neural Network output layer neuron threshold

$\phi \quad$ Roll Euler angle, deg

$\psi \quad$ Yaw Euler angle, deg

$\varphi \quad$ Activation function

\section{Subscripts}
A Aileron
A Aerodynamic
E Elevator
h Index of nodes in input layer
i Index of nodes in hidden layer
m number of nodes in hidden layer
n number of nodes in input layer
$\mathrm{j}$ number of nodes in output layer
L Left side
R Right side
R Rudder
T Thrust
$\mathrm{X} \quad$ Along the $\mathrm{x}$-direction
$\mathrm{y} \quad$ Along the y-direction
z Along the z-direction 


\section{Acronyms}

\begin{tabular}{|c|c|}
\hline A/D & Analog to Digital \\
\hline AFA & Actuator Failure Accommodation \\
\hline AFDI & Actuator Failure Detection and Identification \\
\hline AFDIA & Actuator Failure Detection, Identification, and Accommodation \\
\hline BLS & Batch Least Square \\
\hline BPA & Back Propagation Algorithm \\
\hline C.G. & Center of Gravity \\
\hline CPU & Central Processing Unit \\
\hline CSDS & Control Signal Distribution System \\
\hline DAQ & Data Acquisition \\
\hline DIO & Digital Input/Output \\
\hline DOF & Degree Of Freedom \\
\hline EMI & Electromagnetic Interference \\
\hline FCS & Flight Control System \\
\hline FDI & Failure Detection and Identification \\
\hline FTFCS & Fault Tolerant Flight Control Systems \\
\hline GPS & Global Positioning System \\
\hline HILS & Hardware-In-the-Loop-Simulation \\
\hline HIMAT & Highly Maneuverable Aircraft Technology \\
\hline IDE & Integrated Device Electronics \\
\hline IFCS & Intelligent Flight Control System \\
\hline IMU & Inertial Measurement Unit \\
\hline LED & Light Emitting Diode \\
\hline LSB & Least Significant Byte \\
\hline MIMO & Multi-Input, Multi-Output \\
\hline MLP & Multi Layer Perceptron \\
\hline MMKF & Multiple Model Kalman Filter \\
\hline MSB & Most Significant Byte \\
\hline NN & Neural Network \\
\hline NNC & Neural Network Controller \\
\hline
\end{tabular}




\begin{tabular}{|c|c|}
\hline $\mathrm{OBC}$ & On-Board Computer \\
\hline PAC & Pulse Aperture Correlation \\
\hline PCB & Printed Circuit Board \\
\hline $\mathrm{PCH}$ & Pseudo-Control Hedging \\
\hline PCM & Pulse Code Modulation \\
\hline PI & Proportional plus Integral \\
\hline PID & Parameter IDentification \\
\hline PWM & Pulse Width Modulation \\
\hline $\mathrm{R} / \mathrm{C}$ & Remote Controlled \\
\hline RESTORE & Reconfigurable Control for Tailless Fighter Aircraft \\
\hline RF & Radio Frequency \\
\hline RTW & Real-Time Workshop \\
\hline SAS & Stability Augmentation System \\
\hline SFA & Sensor Failure Accommodation \\
\hline SFDI & Sensor Failure Detection and Identification \\
\hline SFDIA & Sensor Failure Detection, Identification, and Accommodation \\
\hline SIO & Serial Input/Output \\
\hline SISO & Single-Input, Single-Output \\
\hline $\mathrm{S} / \mathrm{N}$ & Signal Noise ratio \\
\hline STD & STandard Deviation \\
\hline $\mathrm{T} / \mathrm{W}$ & Thrust/Weight ratio \\
\hline UAV & Unmanned Aerial Vehicle \\
\hline VG & Vertical Gyro \\
\hline $\mathrm{W} / \mathrm{S}$ & Weight/Wing surface ratio \\
\hline WVU & West Virginia University \\
\hline
\end{tabular}




\section{Chapter 1 Introduction}

With the increased requirement for aircraft reliability, Fault Tolerant Flight Control Systems (FTFCS) with the capabilities for accommodating sensor and actuator failures have become an important focus of study within the aerospace community for a number of years. Related with aircraft system, a fault tolerant flight control system needs to perform the following tasks:

1. Sensor Failure Detection, Identification, and Accommodation (SFDIA);

2. Actuator Failure Detection, Identification, and Accommodation (AFDIA). Ideally, an aircraft control system should have the ability to detect a failure, analyze the degree of damage and attempt to compensate for the failure with the remaining sensors/control surfaces. This research effort focused upon the area of Actuator Failure Accommodation (AFA), which determines on-line which actions and the degree that should be taken by the control system to recover an impaired aircraft. Due to the inherited risk of inducing actuator failure during flight testing, only a limited amount of research activity leading to flight testing has been performed.

The definition of actuator failure may imply a locked control surface, a missing portion of the control surface, or any combination of both. To avoid damaging the test bed aircraft, only locked control surfaces were considered in this research effort. Two failure scenarios were selected for this study:

1. Right Aileron Failure;

2. Right Elevator Failure.

To minimize the risk during flight test phases, a failure was defined as a control surface locked at the trim position. A detailed analysis, controller design, and flight test results will be discussed using the two failure scenarios.

Within a conventional approach to the design of control laws, an accurate mathematic model of the aircraft is required. One critical issue is that during flight tests the aircraft's model is varying throughout the flight envelope. The non-linearity and coupling between the longitudinal and lateral-directional channels also increases the difficulty in designing a linear control law which can handle the entire problem. This difficulty becomes extreme when an actuator failure occurs on the aircraft. Without the 
ability to adapt itself to a changing aircraft model, the linear controller would be ineffective or, at worst, even cause the aircraft to become closed-loop unstable. As an alternative approach, Neural Networks (NNs) were implemented in the flight control system design to allow for an adaptive learning behavior. Two distinct advantages of using NNs in a control system design include:

- Learning ability: the $\mathrm{NN}$ can be trained with past recorded flight data (offline training) or directly trained with the real-time flight data (on-line training);

- Non-linearity: the NN can be trained to approximate a nonlinear system.

With sufficient training, a NN can approximate a nonlinear system with high accuracy [7]. This is very appealing for use with fault tolerant controller design. If properly applied, the use of a NN based system can give a controller the capability to adapt itself with a changing environment. In this study, a set of NNs was integrated into the fault tolerant controller design and installed on the on-board computer for flight test evaluation.

Two training methods can be used for the NN learning: on-line and off-line learning. The off-line learning consists of training with a pre-recorded data set and does not require to be performed in real-time. In this way, off-line training is not restricted by the on-board computer's computation power and the approximation can be highly accurate. At the same time, the off-line learning cannot take the advantage of a neural networks' learning ability to adapt to the changing aircraft dynamics during the flight. The on-line learning method uses the real-time flight data obtained for training and has much higher requirements for on-board hardware and software implementation. However, this method has the ability to "learn" the changing aircraft dynamics and give the controller some level of "intelligence". The on-line NN's learning speed and accuracy is limited by the natural frequency of the system, the on-board computation power and available resources. In this study, both on-line and off-line learning NNs were implemented in the controller design to take the advantage of both approaches.

This project involved both simulation studies and flight testing evaluations. The inherited risk of actuator failure made it a difficult challenge to be tested on a real aircraft. For that reason, Unmanned Aerial Vehicles (UAVs), with their flexibility and 
low cost aspect, became an ideal solution for this type of research. For this project, one of the $3 \mathrm{WVU}$ YF-22 research UAVs was used to perform the final flight testing phases of different AFA schemes to illustrate the level to which handling qualities can be maintained in the presence of failures on the actuation channels. An on-board payload with a PC-104 format computer, instrumentation, and custom made control and connection circuits were designed and developed to perform actuator failure triggering and accommodation during the flight test. Real-time on-board data acquisition and control software was developed with C-language and compiled with Matlab ${ }^{\odot}$ Real-Time Workshop (RTW). A series of flight-tests were then tailored to evaluate the aircraft performance, analyze the mathematical model of the UAV, simulate the failure condition, and demonstrate the ability of an on-board AFA controller.

This research project was a collaboration of a group effort from a number of members of the WVU flight testing research crew. The particular contributions by the author include: design and manufacturing of the on-board electronic payload system; analysis of the actuator failure characteristics; design and simulation of the NN based AFA controller; development of the on-board flight-control software; planning for the AFA flight testing activities; and the post-flight data analysis.

This dissertation is organized as follows: Chapter 2 presents the related work that has been developed by other researchers in the past decade. Chapter 3 is dedicated to the design and simulation of the AFA control scheme. Chapter 4 describes the design and development of the test-bed aircraft and the on-board electronic payload systems. The real-time on-board software is discussed in Chapter 5. Chapter 6 describes the flight testing program and presents the final testing results for the actuator failure accommodation flight tests. A conclusion of the project and recommendations for future works are provided in Chapter 7. 


\section{Chapter 2 Literature Review}

Actuator failure during flight poses a significant flight safety concern and can cause catastrophic results. A passive approach to the problem is based upon hardware redundancy. However, due to the cost and weight requirements for aircraft design, it is not practical to have several redundant control surfaces. A solution from software/controller design provides a low cost solution to aid with this issue. Both traditional gain scheduling and NN based adaptive control methods have been extensively studied in recent years. This section will give a brief overview of techniques developed for actuator failure accommodation purposes.

The aircraft's dynamics is non-linear in nature, especially following actuator failure. However, tools for analysis and design of nonlinear control systems are poorly developed. One solution is to adopt a "divide and conquer" approach whereby the analysis/design task for a nonlinear system is decomposed into a number of simpler linear tasks. Within this approach, gain scheduling has been used for the design of nonlinear control flight systems. The conventional gain-scheduling design approach typically involves three phases:

1. Linearizing the nonlinear plant at a number of equilibrium points;

2. Design a linear controller for each of the plant linearizations;

3. Combine linear controllers to obtain a nonlinear controller.

Several varieties of the gain-scheduling method based on on-line parameter identification [1] had been designed and used in fault tolerance flight control systems. In Shin's work [2], a robust Linear Parameter Varying Control had been designed for Highly Maneuverable Aircraft Technology (HIMAT) vehicle subject to loss of control effectiveness.

The goal of actuator failure accommodation is to maintain or regain the best possible handling qualities in the presence of actuator failures. Conventional gainscheduling methods involve a combination of on-line parameter identification control redesign and/or adaptation for a degraded flight mode. Traditional approaches to flight control reconfiguration requires controller gains to be redesigned in real time. The complications here are substantial since this process requires a reasonably accurate 
knowledge of low frequency aircraft dynamics. Gain scheduling has been a very successful approach in flight control system design without failure conditions. One drawback of this methodology in a fault tolerance system is that it depends on a predetermined set of failure models, making it less effective in case of failures that have not been previously modeled. Also, the number of required gains, which have to be designed and scheduled can also be very large. Extensive off-line analysis, in-flight tuning, and validation of gain schedules are necessary to handle a large set of possible failure modes.

In recent years, several theoretical developments have lead towards the use of neural networks that learn and adapt on-line for nonlinear systems [3][4][5]. The main advantage lies in eliminating the need for a detailed Parameter IDentification (PID) during the recovery phase, and limiting the potential need for PID in the problem of gain rescheduling following a failure. In general, the need for an accurate aerodynamic database for the purpose of flight control design can be significantly reduced through the use of a NN-based approach.

NNs have been applied very successfully in the identification and control of dynamic systems [6]. The universal approximation capabilities [7] of the MultipLayer Perceptron (MLP) have made it a popular choice for modeling nonlinear systems and implementing general-purpose nonlinear controllers [8]. NN based flight control systems are designed to provide adaptive flight control without requiring extensive gain scheduling or explicit system identification. Most NN based controllers are developed from conventional controller architecture, including Fixed Stabilizing Control [9], Nonlinear Internal Model Control [10], Model Predictive Control [11], Model Reference Control [12], Adaptive Critic Control [13] and Adaptive Inverse Control [14].

$\mathrm{NN}$ augmented adaptive inverse control has been extensively studied for use with flight control systems. A NN based autopilot system, incorporating direct adaptive control with dynamic inversion, has been used in John Kaneshige's work [8]. Feedback Linearization Augmented with $\mathrm{NN}$ inverse dynamic approximator was proposed in a $\mathrm{X}$ 33 Reusable Launch Vehicle controller design [15][16][17].

A NN based nonlinear inverse dynamic controller was flight tested with model helicopter at the Georgia Institute of Technology [18]. A methodology called Pseudo- 
Control Hedging (PCH)[19] was employed to protect the system from the adverse affects of incorrect adaptation in the event of actuator saturation and failure.

The performance analyses for the $\mathrm{NN}$ based controller have also been extensively studied. Thompson [20] discussed the performance analysis in the frequency domain in his work. The controllability and stabilization analysis for the NN controller were also discussed in [21][22][23].

Different types of activation function and training methods can lead to different approximation performance and learning speed. Radial Basis Function [24] and extended back-propagation training [25] had been studied for the aircraft controller design.

Due to the extreme risk of the actuator failure in flight, so far only very limited flight testing has been performed. In fact, almost no NN based AFA scheme have been fully evaluated in flight. Most of the related studies were theoretical or simulation based. Two-research efforts with both actuator failure study and NN based flight controller are similar to this research project: the Reconfigurable Control for Tailless Fighter Aircraft (RESTORE) program [26-29] and the Intelligent Flight Control System (IFCS) project [30-40]. A brief description of these two research activities will now be introduced.

The purpose of the RESTORE program was to develop and evaluate reconfigurable flight control algorithms. Unlike traditional reconfiguration methods, RESTORE control laws were designed to compensate for unknown aircraft damage, as well as actuator failures, by adapting to the changing aircraft dynamics.

The Boeing RESTORE team designed the controller based on the dynamic inversion (Figure 2-1). The NN was developed to adaptively regulate the inversion error between the pre-estimated aircraft model and the true aircraft dynamics. The inversion error can be caused by the model uncertainty, actuator failure, or aircraft damage. A control allocation module was used to distribute the desired control response from the control algorithms to the remaining "healthy" control actuators. A system identification module uses a Least Squares (LS) algorithm to estimate aerodynamic parameters. Null space injection is used to briefly excite control surfaces to obtain these estimates without significant performance degradation [27]. 


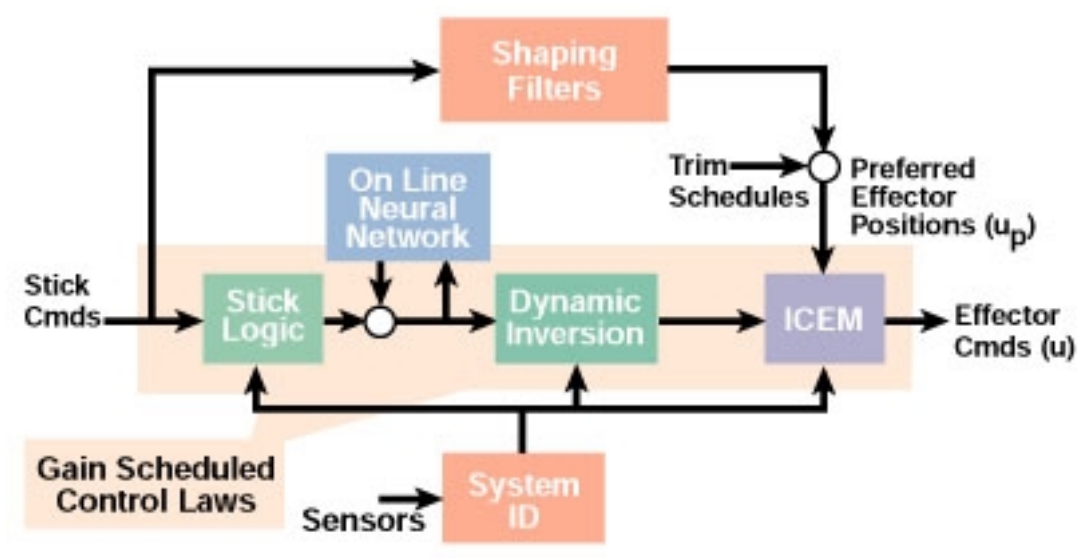

Figure 2-1 RESTORE Controller

The test-bed aircraft for the RESTORE project was the X-36 jointly sponsored by the AFRL and NAVAIR. This aircraft features redundant, multi-axis conventional control surfaces, split flaps, and yaw thrust vectoring to provide reconfiguration capability for simulated control surface failures.

A NN was integrated into the existing X-36 flight control system. Piloted hardware-in-the-loop-simulation (HILS) testing was used to mature the reconfigurable control laws and evaluate their robustness for a variety of simulated actuator failures. The control laws were given no prior knowledge of the simulated failure input. While performing the HILS testing, the RESTORE control laws were compared to the baseline flight control laws.

Two RESTORE research flights were flown in December 1998, proving the viability of the software approach. A wing trailing-edge control surface failure was triggered during the flight testing. In addition to the limited flight testing completed in December 1998, the RESTORE technology was demonstrated using aircraft piloted simulations in the summer of 1999 [28].

The IFCS flight research project at NASA Dryden Flight Research Center was established to design aircraft flight controls that can optimize aircraft performance in both normal and failure conditions. IFCS was designed to incorporate self-learning NN concepts with different purposes and levels of criticality into the flight control software to enable a pilot to maintain control and safely land an aircraft that has suffered a major systems failure and/or combat damage [30]. 
The test-bed aircraft for the IFCS project is the NASA NF-15B (NASA 837.). This aircraft has been highly modified from a standard F-15 configuration to include canard control surfaces, thrust vectoring nozzles, and a digital fly-by-wire flight control system. The IFCS GEN I concept (Figure 2-2) is a direct adaptive NN approach.

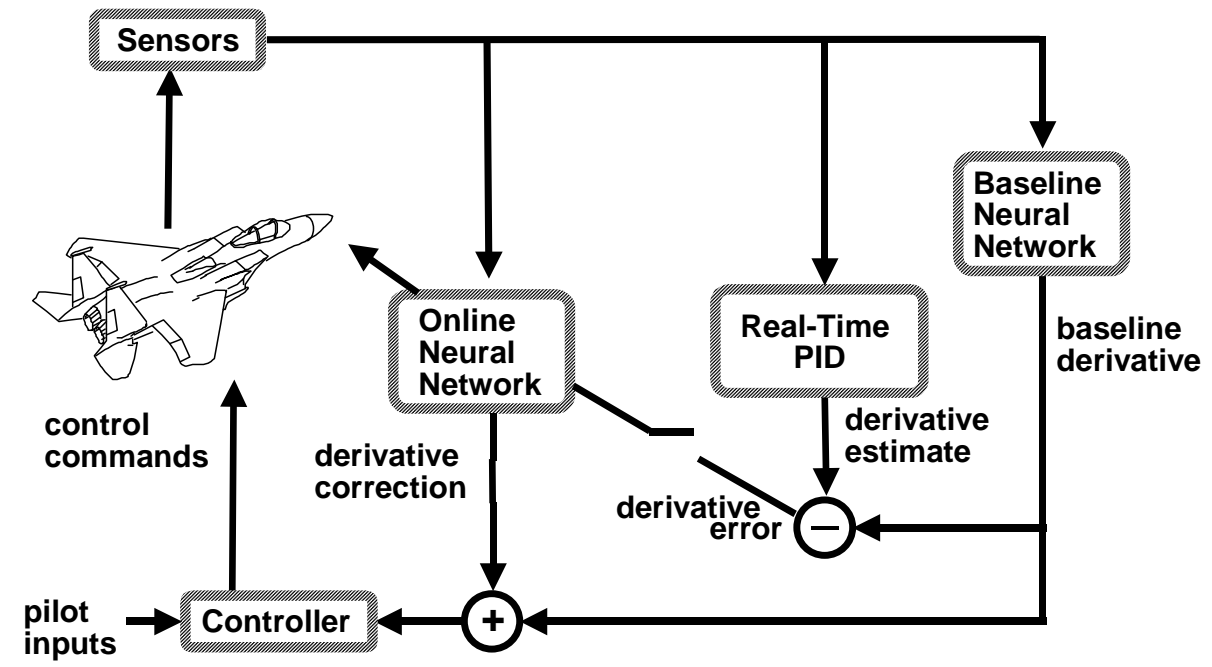

Figure 2-2 General Block Diagram of the IFCS GEN I Controller

GEN I was designed to estimate the aircraft aerodynamic changes caused by simulated failure modes, and provides the information to the flight control system. The system identifies dynamic characteristics of the vehicle, with the form of the stability and control derivatives, and uses them to stabilize the vehicle and provide specific flying characteristics. Particularly, the updated values of the main stability derivatives with respect to baseline values - through NN-based mapping - are fed to an optimal controlbased set of control laws.

The IFCS GEN II NNs are designed to take over more direct control of the aircraft by working with the flight controller to compensate for any shortcomings. The NNs are an integral component of the control laws in the GEN-II architecture instead of serving as "mapping" tools in the Gen I architecture.

The GEN II concept is based on a dynamic inversion controller with a modelfollowing command path shown in Figure 2-3. 


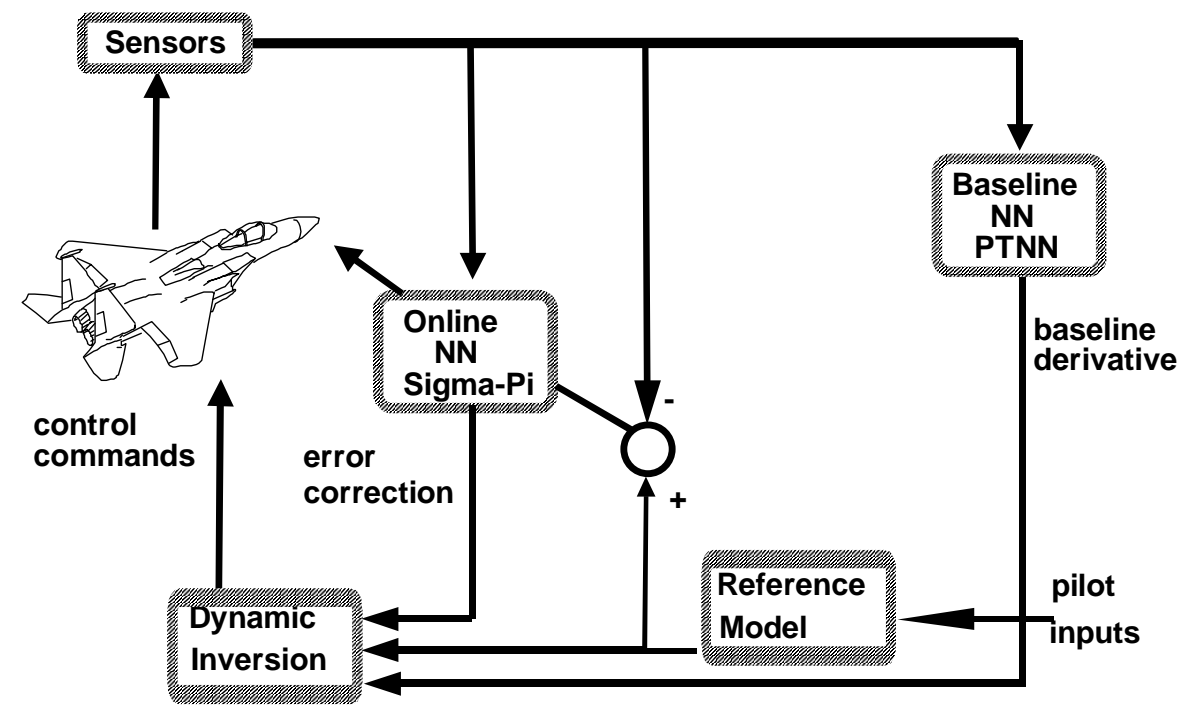

Figure 2-3 General Block Diagram of the IFCS GEN II Controller

The feedback errors are controlled with a Proportional + Integral (PI) controller. This basic system is then augmented with an adaptive $\mathrm{NN}$ which operates directly on feedback errors. The adaptive $\mathrm{NN}$ adjusts the system for miss-predicted behavior or changes in behavior caused by the damage. Demonstration of this direct adaptive NN is the primary objective of the IFCS GEN II flight project [32].

The dynamic inversion portion of the control system generates acceleration commands. These commands are translated into control surface commands by a control allocation scheme. The dynamic inversion, control allocation, and model-following algorithm all require information on the dynamic behavior of the system to be controlled. This information is then provided in the form of aircraft aerodynamic stability and control derivatives [33].

Initial PID test flights with an IFCS NN that was pre-trained to the F-15's aerodynamic database were flown in 1999. A follow-up series of flights are being flown in the summer of 2002 to calibrate new instrumentation and air data systems and repeat several of the test points flown in the 1999 series to reduce risk for the GEN I and II flight research phases.

The IFCS GEN I flight testing has been suspended to be concentrated on the GEN II program. GEN II flight testing preparations are current underway. West Virginia University has been involved in the IFCS GEN II controller design and flight testing as 
well as the design of a "safety monitor" scheme to allow smooth and safe transitions from conventional to research control laws and from research control laws at nominal conditions to failure conditions. Furthermore, within the activities of an additional project, one of the WVU YF-22 Research Aircraft Model will be used to test a set of IFCS control laws as a scaled-down version of the IFCS flight testing program. 


\section{Chapter 3 AFA Controller Design}

The design of the AFA controller represents a difficult and challenging problem. These difficulties arise from the changing and uncertainties associated with the aircraft dynamics following a failure occurrence. In a conventional fault tolerance controller design, extensive knowledge of the aircraft dynamics after failure is required. However, this is not usually practical with numerous actuator failure conditions. Therefore, a set of control laws with self-learning ability would be preferred. In this project, two failure modes were simulated during the flight testing phases, that is: right elevator failure and right aileron failure. The main objective of the effort was to demonstrate actuator failure accommodation using both simulation and flight testing results. Neural networks were selected in the controller design to provide the learning ability. To simplify the problem, we assume that the actuator failure is detected and identified instantly. Developed from the overall program goals laid out in Chapter one, the following requirements were defined for the AFA controller design:

- The NN learns on-line to approximate the aircraft's response, especially after the actuator failure;

- The controller uses the estimation of the aircraft dynamics from the NN and adjusts the control command to accommodate for the failure;

- The learning process is required to be as short as possible to compensate for the fast changing aircraft dynamics;

- The NN needs to be designed and implemented with limited on-board computation resources;

- Stability for the closed-loop system after injection of the failure.

In this chapter, the design of a $\mathrm{NN}$ based AFA controller will be presented followed by the detailed design process: the type and dimension of the NN which was selected to approximate the aircraft dynamics; a linear mathematic model of the test-bed aircraft acquired from flight test data; a linear controller was designed at a nominal flight condition as the base-line control system; according to the different failure types, the effect of the actuator failure will be analyzed and AFA controllers are designed accordingly with detailed simulation analysis. 
The AFA controller design, as a special case for the flight control system, had to be closely associated with the flight testing program. Preliminary flight tests were performed for each of the controller design phases for analyzing the aircraft performance and failure conditions. In this chapter, the flight testing data used for designing the AFA controller will be presented along with the simulation study. The flight testing data for the final demonstration of designed AFA controllers will then be presented in Chapter 6 .

\section{1 - Neural Network based Controller Design}

Artificial NNs in general can be defined as mathematical models of human brain activities. Typically, NNs consist of many simple processing units that are wired together in a complex communication network. Each unit, or node, is a simplified model of a "real" neuron which sends a new signal if it receives a sufficiently strong input signal from the other nodes to which it is connected. The strength of these connections may be varied in order for the network to perform different tasks corresponding to different patterns of node firing activity. The simplest definition of a neural network, is provided by the inventor of one of the first neurocomputers, Dr. Robert Hecht-Nielsen. He defines a neural network as: "... a computing system made up of a number of simple, highly interconnected processing elements, which process information by their dynamic state response to external inputs" [41].

NNs have been widely applied in controller designs over the past decade. In a neural network based controller, the NN typically has been trained to approximate either the dynamics or inverse dynamics of a plant. While trained with the inverse dynamics of a plant, the $\mathrm{NN}$ is normally used to cancel out any nonlinear dynamics (nonlinear dynamic inversion). Since the inverse dynamics of the plant is often unstable, it is difficult to train a $\mathrm{NN}$ so that it can approximate it reliably. If the $\mathrm{NN}$ cannot approximate the inverse dynamics of the system to a certain degree of accuracy, the stability of the control system cannot be guaranteed. Several types of NN controllers based upon the inverse dynamics had been designed and simulated in early phases of this project but eventually abandoned because of the learning difficulties. NNs trained to approximate the full or a portion of the aircraft dynamics was used in the final AFA controller design. 
To overcome the linear controller's shortcomings to accommodate for the timevarying dynamics of the aircraft, a new NN based controller structure was designed based upon a linear feedback control system. The general architecture of the controller is shown in Figure 3-1.

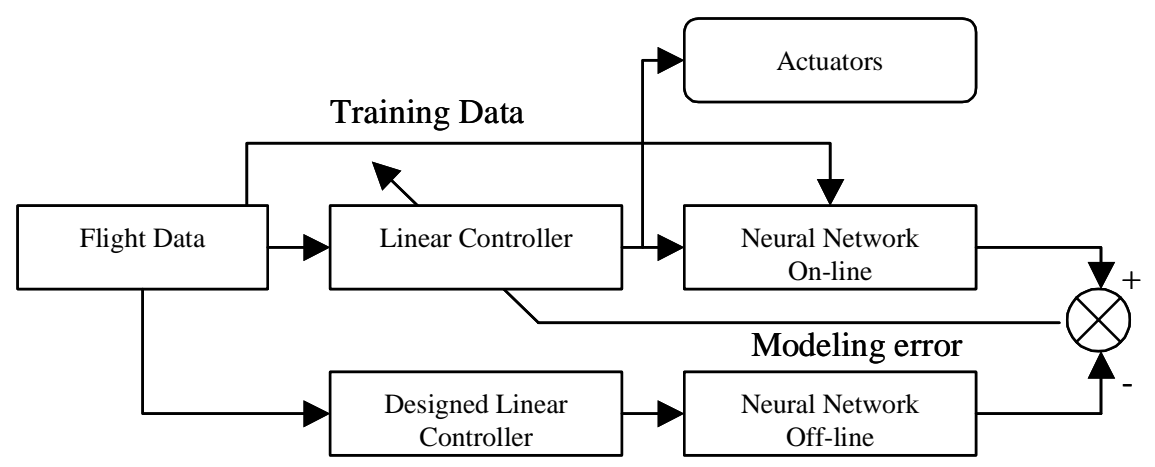

Figure 3-1 Architecture of the NN Controller

In this design, a linear mathematical model of the aircraft at a normal flight condition was required and a linear controller design was based upon the mathematical model to stabilize the aircraft at the normal flight condition. Two sets of NNs with the same structure are used in the controller - On-line Learning NN and Off-line Learning NN. Both NNs are pre-trained with flight data acquired from the nominal flight condition. During the take-off and landing stages of the flight testing, the learning of the $\mathrm{NN}$ is turned off since the flight data would be relative to different flight conditions. In this case, both NNs will have exactly the same weights and thresholds so that they will provide equal estimations. Once the aircraft reaches a nominal flight condition, the online $\mathrm{NN}$ begins training while the off-line $\mathrm{NN}$ stays constant and provides a reference approximation of the aircraft. If the aircraft model had been changed, the modeling error can then be calculated with the difference of both $\mathrm{NN}$ estimations. In the event of actuator failure, the on-line learning $\mathrm{NN}$ will be trained to approximate the aircraft dynamics after the failure while the off-line learning $\mathrm{NN}$ will remain to provide estimation of the aircraft dynamics under a nominal flight condition (without failure). This estimation difference indicates the change of the aircraft dynamics and can be used to tune the linear feedback control gains. In this way, the controller has the capability to learn and adapt to the different flight conditions - including actuator failures - by 
redesigning the linear feedback gains on-line to compensate for the changing aircraft model.

\section{2 - Neural Network Selection}

Based upon the controller design concept and requirements of flight testing, the selected NNs must have the following capabilities:

- Ability to approximate the nonlinear aircraft dynamics;

- Capable of on-line learning;

- Fast learning;

- Require minimal memory and computation power.

With these requirements, a Multilayer Perceptron (MLP) network with a backpropagation training algorithm was selected as the NN estimator. A MLP is a network of simple neurons called perceptrons. The basic concept of a single perceptron was introduced in 1958 by Rosenblatt. The perceptron computes a single output from multiple real-value inputs by forming a linear combination according to its input weights and then putting the output through some nonlinear activation function. Mathematically this can be described using:

$$
\begin{gathered}
u_{k}=\sum_{j=1}^{p} w_{k j} x_{j} \\
y_{k}=\varphi\left(u_{k}-\theta_{k}\right)
\end{gathered}
$$

where $x_{1}, x_{2} \ldots x_{p}$ are the inputs to each neuron in the input layer; $w_{k 1}, w_{k 2} \ldots . w_{k p}$ are the synaptic weights associated with each input. The value $u_{k}$ is the output of the linear combiner; $\theta_{k}$ is the threshold value with $\varphi()$ being the activating function and $y_{k}$ being the final output of the individual neuron.

The most used activation function for a MLP is the sigmoid function. Based upon a bipolar characteristic of the sensor signal in the YF-22 research UAV's on-board payload, the activation functions selected for this project was the "tansig" function $E q(3$ 3) for the hidden layer. A linear output layer was also used to provide a larger output range. Thus, the activation functions for the two layers are given by: 


$$
\text { Hidden Layer: } \quad \varphi(v)=\frac{2}{1+e^{-2 v}}-1
$$

$$
\text { Output Layer: } \quad \varphi(v)=v
$$

The backpropagation algorithm was created by generalizing the Widrow-Hoff [42] learning rule to multiple-layer networks and nonlinear differentiable transfer functions. Input vectors and corresponding output vectors are used to train the network until it can approximate a function. Networks with biases, a "tansig" hidden layer, and a linear output layer are capable of approximating a nonlinear function with a finite number of discontinuities [7].

Standard backpropagation is a gradient descent algorithm. The term backpropagation refers to the manner in which the gradient is computed for nonlinear multilayer networks [84]. The purpose of this rule is to minimize a cost function based upon the error:

$$
e_{k}(t)=d_{k}(t)-y_{k}(t)
$$

where $d_{k}(t)$ is the desired output from the network and $y_{k}(t)$ is the actual response of the network, to the input presented, such that the actual response of each neuron in the output layer approaches the target response in some statistical sense.

The error-backpropagation training process consists of two distinct phases, namely a forward phase and a backward phase. In the forward phase, an input pattern is applied to the nodes in the input layer, which are then propagated through each of the hidden layers until it reaches the output layer, where then the output of the computational nodes are the overall response of the network to the input pattern presented. Once the overall response of the network is obtained, the response is compared to the target output and differences between the two produces an error term. This error term is then propagated backwards, leading to the term backpropagation through the network structure and the corresponding interconnection weights are adjusted to make the response of the network move closer to the desired value.

In the forward phase, the output of hidden layer neurons can be calculated with $E q(3-6)$ and (3-7) 


$$
\begin{gathered}
\left.v_{b i}\right|_{k}=\left.\sum_{h=1}^{n}\left(a_{h} w_{h i}\right)\right|_{k}-\left.\theta_{b i}\right|_{k} \\
\left.b_{i}\right|_{k}=\frac{2}{1+e^{-\left.2 v_{b_{i}}\right|_{k}}}-1
\end{gathered}
$$

where $\left.b_{i}\right|_{k}$ is the value of the $i_{\text {th }}$ hidden neuron at step $\mathrm{k}$. The value of output layer neurons can be calculated with $E q(3-8)$ and $E q(3-9)$. From the hidden to the output layer:

$$
\begin{gathered}
\left.v_{c i}\right|_{k}=\left.\sum_{i=1}^{p}\left(b_{i} w_{i j}\right)\right|_{k}-\left.\theta_{c j}\right|_{k} \\
\left.c_{j}\right|_{k}=v_{c j}
\end{gathered}
$$

where $\left.c_{j}\right|_{k}$ is the value of the $\mathrm{j}_{\mathrm{th}}$ output neuron at step $\mathrm{k}$. Through the application of the steepest descent method as an iterative scheme to minimize the mean-squared error, the following is calculated:

for the output layer, at time $\mathrm{k}$

$$
\left.\frac{\partial \varphi\left(v_{c j}\right)}{\partial v_{c j}}\right|_{k}=\left.\varphi_{v_{c j}}^{\prime}\right|_{k}=1
$$

for the hidden layer, at time step " $\mathrm{k}$ "

$$
\left.\frac{\partial \varphi\left(v_{b i}\right)}{\partial v_{b i}}\right|_{k}=\left.\varphi_{v_{b i}}^{\prime}\right|_{k}=1-\left.\varphi_{v_{b i}}^{2}\right|_{k}
$$

Next, at time $\mathrm{k}$, a $\Delta$ term is calculated at the output layer:

$$
\left.\delta_{v_{c j}}\right|_{k}=\left.\left.\left(\bar{c}_{j}-c_{j}\right)\right|_{k} \varphi_{v_{c j}}^{\prime}\right|_{k}=\left.\left(\bar{c}_{j}-c_{j}\right)\right|_{k}
$$

where this term can be used to update output layer weights and thresholds. The $\Delta$ term evaluated for the hidden layer are calculated using $E q(3-13)$

$$
\left.\delta_{v_{b i}}\right|_{k}=\left.\varphi_{v_{b i}}^{\prime}\right|_{k}\left(\left.\left.\sum_{i=1}^{q} w_{i j}\right|_{k} \delta_{v_{c j}}\right|_{k}\right)=\left(1-\left.\varphi_{v_{b i}}^{2}\right|_{k}\right)\left(\left.\left.\sum_{i=1}^{q} w_{i j}\right|_{k} \delta_{v_{c j}}\right|_{k}\right)
$$

this term can then be used to update the hidden layer weights and thresholds.

In the designed AFA controller, NNs are used to approximate the dynamics of the research aircraft model. An example of NN design and simulation to approximate the lateral-directional dynamics of the YF-22 model aircraft is shown below. The real flight 
data was used for training the $\mathrm{NN}$ includes both the nominal flight condition and flight with aileron failures. This particular NN was used in the aileron failure AFA controller design which will be described in Section 3.5. To approximate the lateral-directional dynamics of the aircraft, several states of the aircraft were required, including:

- aileron deflection;

- rudder deflection;

- $\quad$ angle of Sideslip;

- roll rate;

- yaw rate.

The inputs of the NN at step $\mathrm{k}$ been selected is shown in Table 3-1:

\begin{tabular}{|l|l|l|l|l|}
\hline$\delta_{\mathrm{a}}(\mathrm{k})$ & $\delta_{\mathrm{r}}(\mathrm{k})$ & & & \\
\hline$\delta_{\mathrm{a}}(\mathrm{k}-1)$ & $\delta_{\mathrm{r}}(\mathrm{k}-1)$ & $\beta(\mathrm{k}-1)$ & $\mathrm{P}(\mathrm{k}-1)$ & $\mathrm{R}(\mathrm{k}-1)$ \\
\hline$\delta_{\mathrm{a}}(\mathrm{k}-2)$ & $\delta_{\mathrm{r}}(\mathrm{k}-2)$ & $\beta(\mathrm{k}-2)$ & $\mathrm{P}(\mathrm{k}-2)$ & $\mathrm{R}(\mathrm{k}-2)$ \\
\hline
\end{tabular}

Table 3-1 Inputs of the Lateral-Directional Neural Networks

$\beta(\mathrm{k}), \mathrm{P}(\mathrm{k})$ and $\mathrm{R}(\mathrm{k})$ signals were used to evaluate and train the neural network:

To guarantee the on-line training speed and minimize the overfitting problems, the size of the hidden layer was designed to be small, leading to a total of 5 hidden neurons. In this way, the structure of the NN includes:

- 12 input neurons;

- 5 hidden neurons;

- 3 output neurons.

The NN was pre-trained (batch training) with flight data acquired from earlier flight tests without failure. The last 20 seconds of the flight data was allocated for evaluation, which had not been included in the training sets of data. The training error is shown in Figure 3-2: 


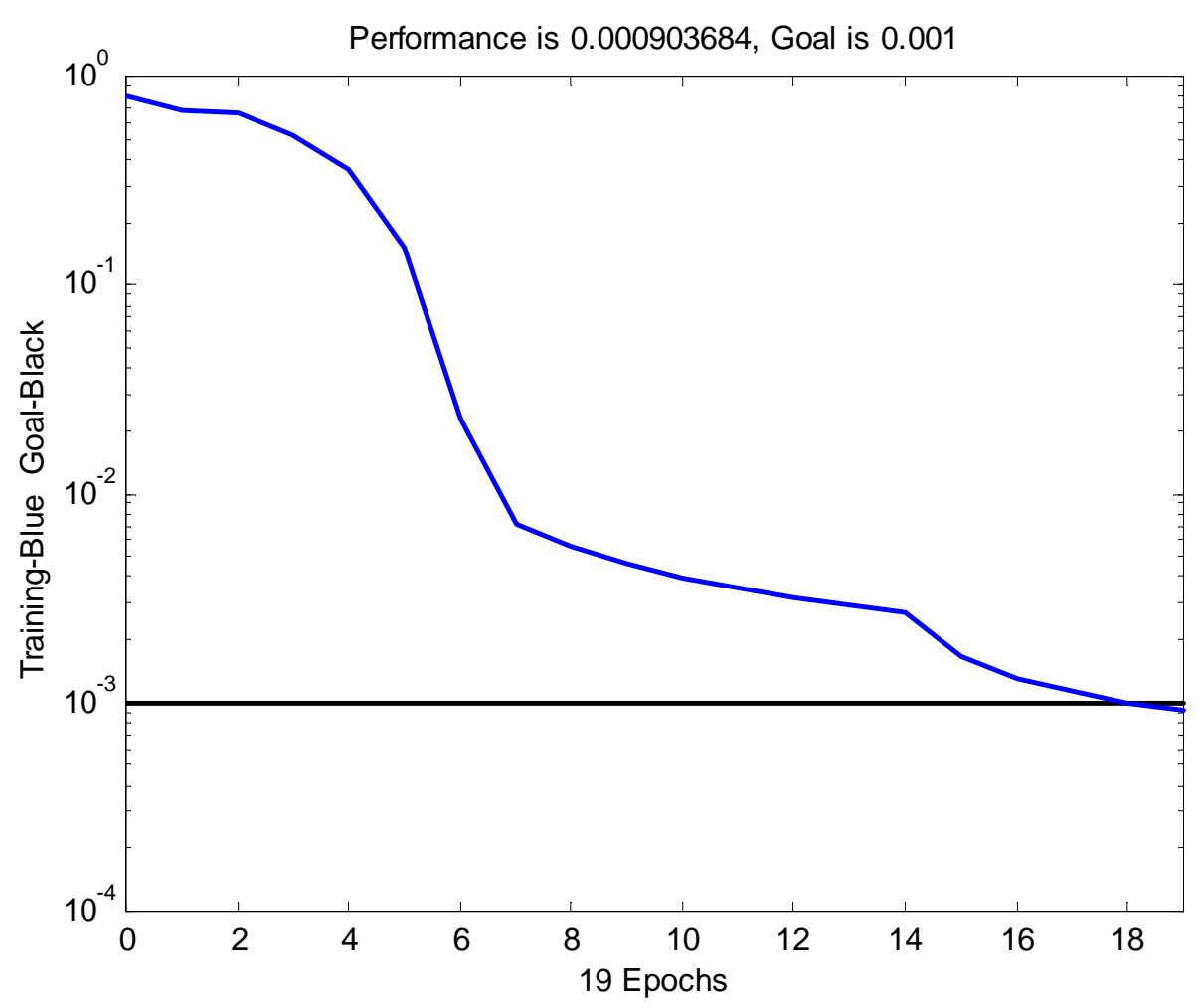

Figure 3-2 Neural Networks Training

After 19 epochs of training, the output of the NN started to show a satisfactory performance in approximating the aircraft dynamics and the training error had been decreased to be under 0.001 . Given the input of the aileron and rudder deflections, it can approximate flight data of the YF-22 model aircraft to a high degree of accuracy. The evaluations of the $\mathrm{NN}$ training with the last 20 -seconds of flight data are shown in Figures 3-3 and 3-4. 


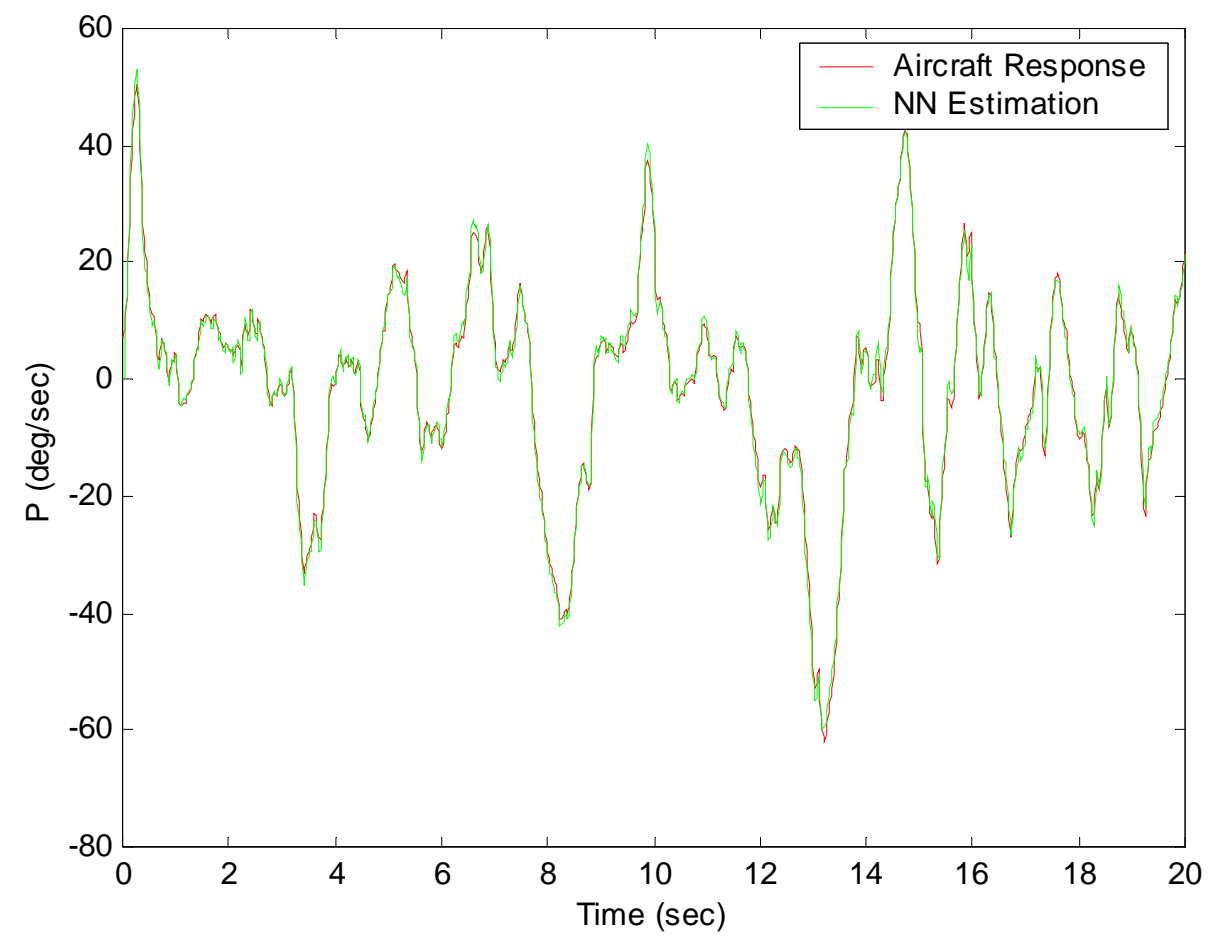

Figure 3-3 Neural Networks Evaluation P-Channel

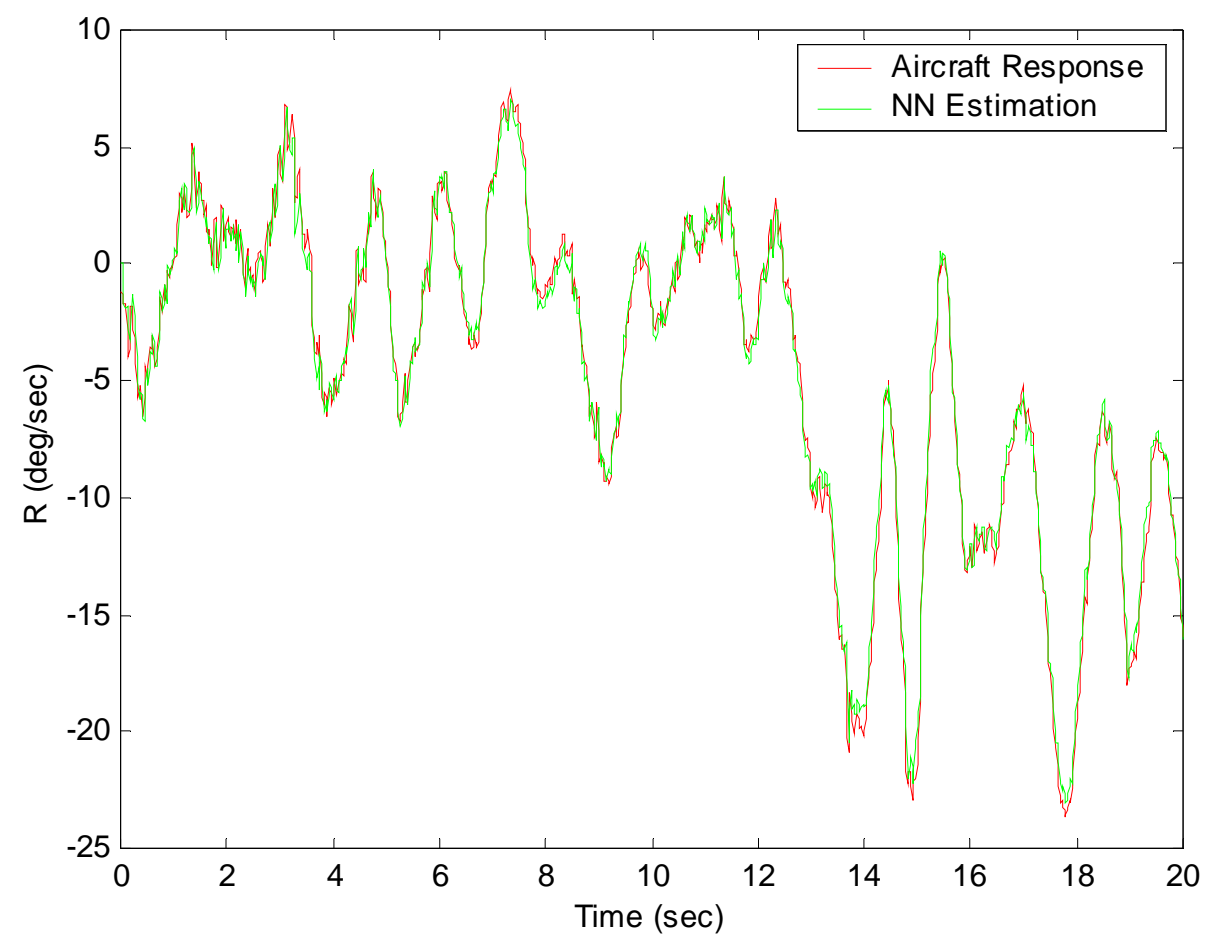

Figure 3-4 Neural Networks Evaluation R-Channel 
The standard deviation of the estimation error for the P and R channels were $1.32 \mathrm{deg} / \mathrm{sec}$ for roll rate and $0.6451 \mathrm{deg} / \mathrm{sec}$ for yaw rate. The pre-trained $\mathrm{NN}$ was then used to estimate the flight data with the right aileron failure (Oct.31 2002 Flight \#2 Failure \#1). The estimation results are shown in Figure 3-5 and 3-6.

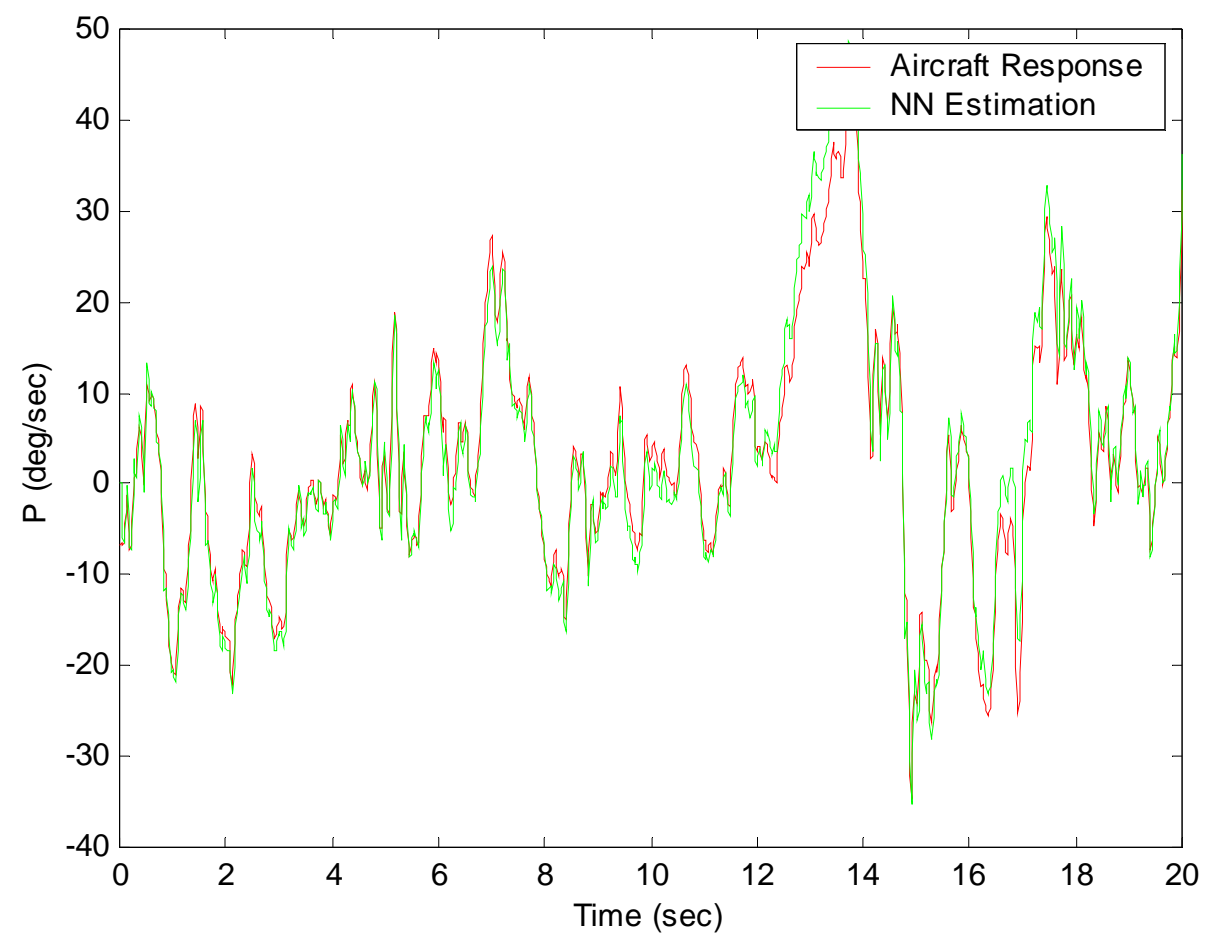

Figure 3-5 Neural Networks Evaluation P-Channel (w/failure) 


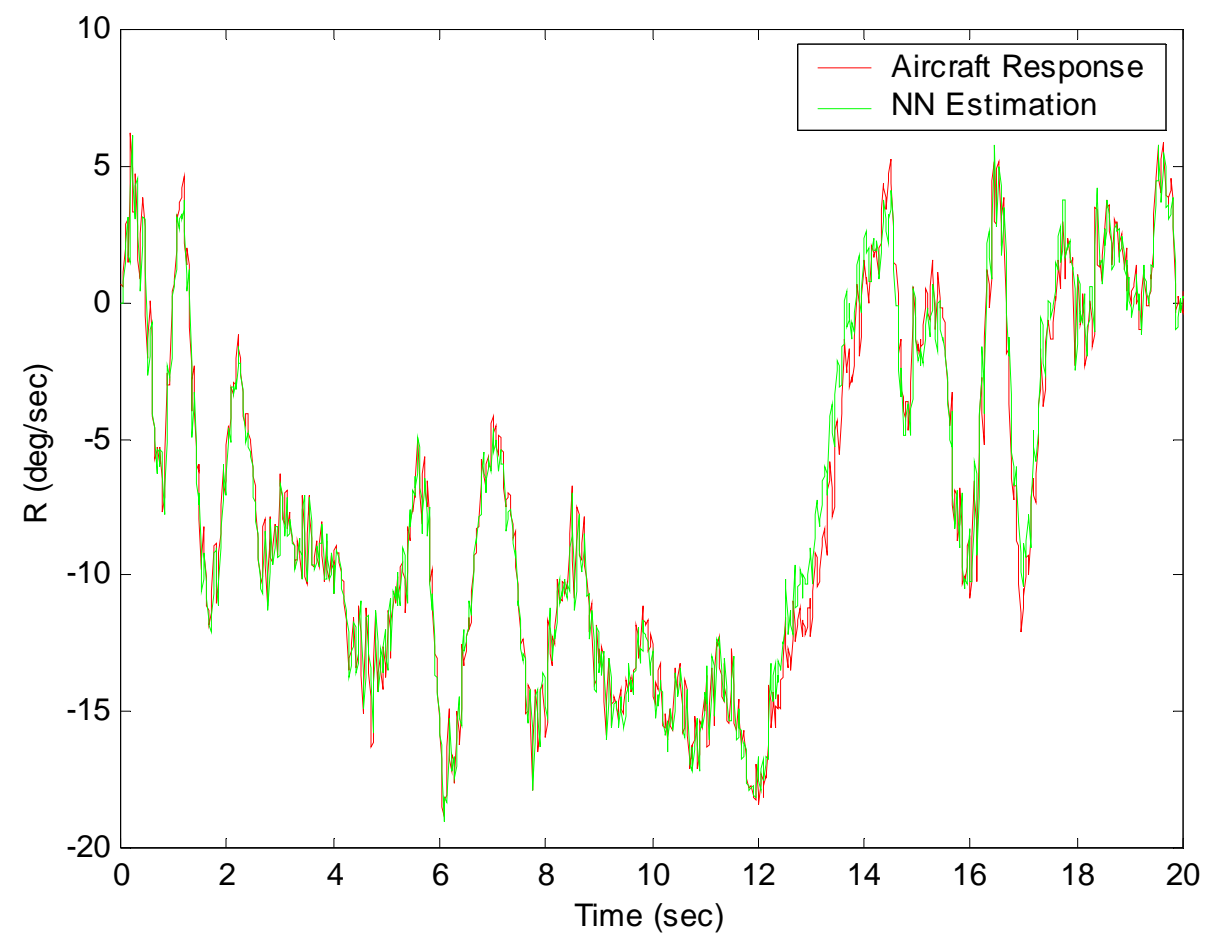

Figure 3-6 Neural Networks Evaluation R-Channel (w/failure)

The standard deviation of the estimation error on the $\mathrm{P}$ and $\mathrm{R}$ channels were 2.6773 $\mathrm{deg} / \mathrm{sec}$ and $1.0238 \mathrm{deg} / \mathrm{sec}$ respectively. The estimation results show that the lateraldirectional dynamics of the aircraft model were changed after the right aileron had been locked at a trim position. The $\mathrm{NN}$ trained with the flight data under nominal flight conditions could not approximate it without additional on-line training.

To compensate for the changing aircraft dynamics, the on-line incremental learning of the NN had been turned on. The estimation results are shown in Figures 3-7 and 3-8. The standard deviation of the estimation error on the $\mathrm{P}$ and $\mathrm{R}$ channels were $1.6021 \mathrm{deg} / \mathrm{sec}$ and $0.9296 \mathrm{deg} / \mathrm{sec}$ respectively. Therefore, with the on-line training, the NN was able to learn the change of the aircraft dynamics and provide an improved approximation after the failure occurs. 


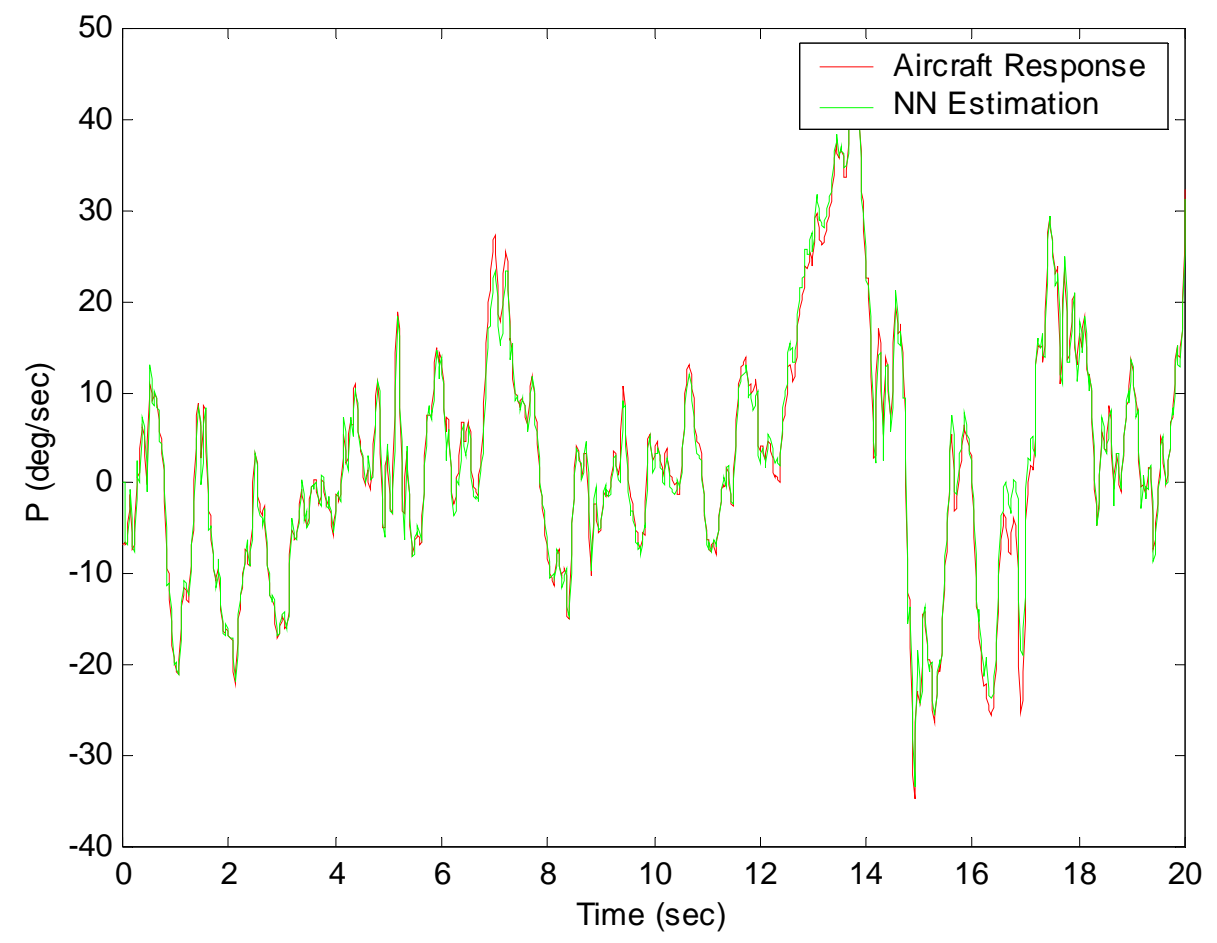

Figure 3-7 Neural Networks Estimation of Actuator Failure P-Channel (w/ on-line training)

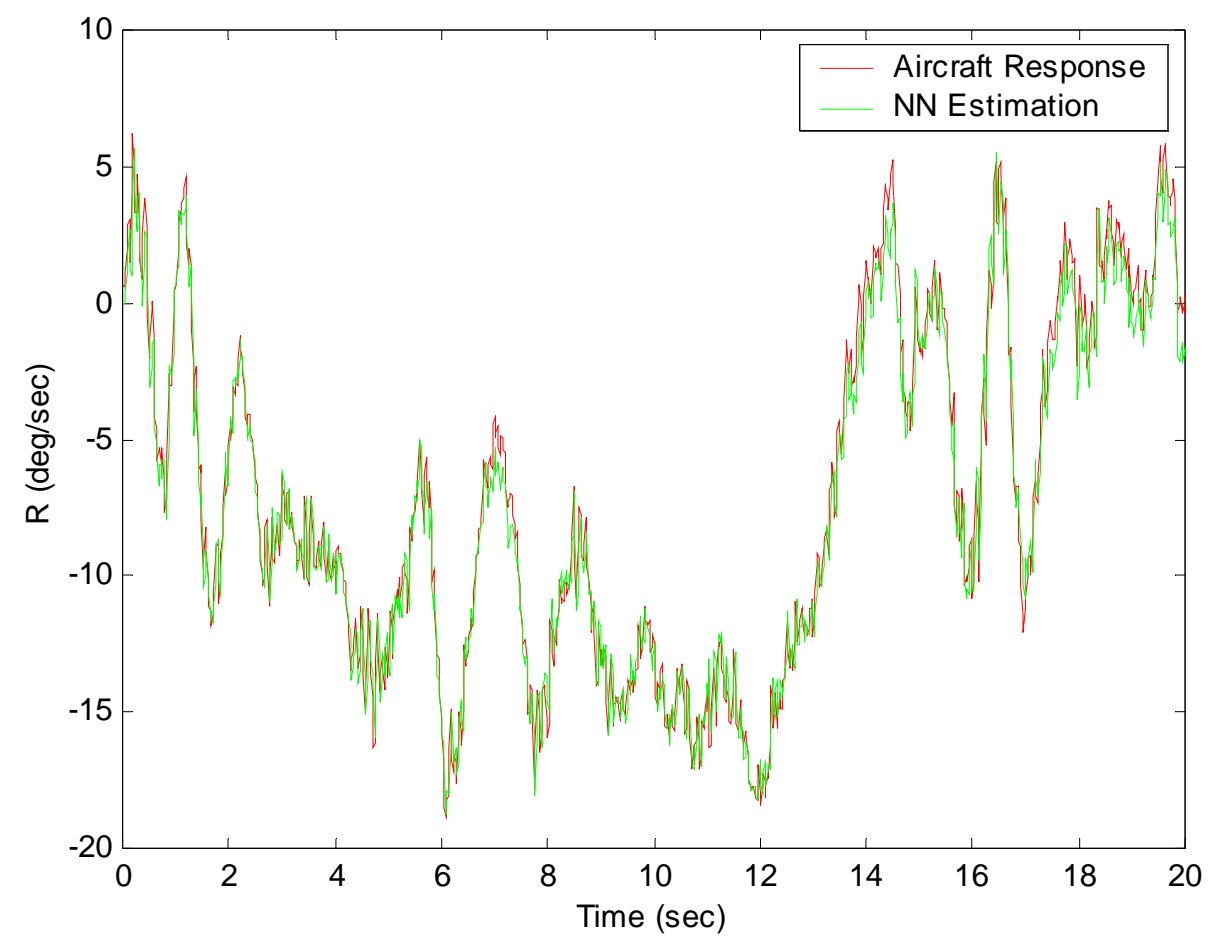

Figure 3-8 Neural Networks Estimation of Actuator Failure $R \_C h a n n e l$ (w/ On-line Training) 
From the above simulation with the YF-22 flight data, the following conclusions can be derived:

- Selected NNs can approximate the non-linear dynamics of the aircraft;

- Lateral dynamics of the YF-22 research UAV were changed after the right aileron locked at the trim position;

- A NN estimator can be used to detect this change;

- On-line learning gives the on-board NNs the ability to adapt the new dynamics of the aircraft after the actuator failure.

With the on-line learning NN's ability to approximate and adapt to the changing dynamics of the aircraft, the AFA on-board controller was designed to accommodate for actuator failures of the YF-22 model aircraft.

\section{3 - Linear Mathematical Model}

According to the selected AFA controller design, a linear mathematical model for the aircraft was required for designing the linear feedback controller under nominal flight conditions. Flight tests were performed to collect data for model identification purposes. Special maneuvers, including elevator doublets, aileron doublets, rudder doublets and a combination of aileron/rudder doublets were performed to fully excite the aircraft's longitudinal and lateral-directional dynamics. A Batch Least Squares (BLS) technique was used to estimate the parameters to obtain a linear mathematical model.

The method of least squares assumes that the best-fit curve of a given type is the curve that has the minimal sum of the deviations squared (least square error) from a given set of data [85]. The BLS technique consists essentially in solving an overdetermined linear system in a "least square" sense. It is one of the most widely used approaches for the estimation of a vector of parameters from a collection of "almostlinearly" related input-output data. In other words, the reliability of this method comes from the property that a pseudo-inverse solution for a linear system with more equations than unknowns is optimal in the least squares sense. The general linear regression model is given by:

$$
Y=X \beta+\varepsilon
$$


where $Y$ is a $(n \times 1)$ vector of known responses of the system, $X$ is a $(n \times p)$ matrix of known inputs to the system. $\beta$ is the $(p \times 1)$ vector of parameters to be estimated, and $\varepsilon$ is a $(n \times 1)$ vector of independent normal random variables, with zero mean $(E\{\varepsilon\}=0)$ and unknown diagonal variance-covariance matrix. This matrix is generally assumed to be a multiple of the $(n \times n)$ identity matrix: $\left(\sigma^{2}\{\varepsilon\}=\sigma^{2} I\right)$. Therefore, we have $E\{Y\}=X \beta$ and $\sigma^{2}\{Y\}=\sigma^{2} I$. The problem is to find the vector $\beta$ such that $X \beta$ (which is the expected value of $Y$ ) is as close as possible (in the least squares sense) to $Y$, so that $\sigma^{2}$ is minimized. Particularly, the objective is to find a value of $\beta$ that minimizes the following quadratic index:

$$
Q=\varepsilon^{T} \varepsilon=(Y-X \beta)^{T}(Y-X \beta)
$$

For the purposes of this effort, the goal is to identify a linear system of the following form:

$$
\left[\begin{array}{l}
\dot{x}(t) \\
y(t)
\end{array}\right]=\left[\begin{array}{ll}
A & B \\
C & D
\end{array}\right]\left[\begin{array}{l}
x(t) \\
u(t)
\end{array}\right]
$$

By transposing $E q(3-16)$ one can define the following:

$$
\begin{aligned}
& Y=\left[\begin{array}{ll}
\dot{x}^{T}(t) & y^{T}(t)
\end{array}\right] \\
& X=\left[\begin{array}{ll}
x^{T}(t) & u^{T}(t)
\end{array}\right] \\
& \beta=\left[\begin{array}{ll}
A & B \\
C & D
\end{array}\right]^{T}
\end{aligned}
$$

A linear aircraft model can usually be considered as a decoupled longitudinal and lateraldirectional sub-models. Both longitudinal and lateral-directional sub-models are normally considered as the linearized models from the nonlinear model which trimmed at a steady-state condition of straight-level flight with nominal airspeed (about $42 \mathrm{~m} / \mathrm{s}$ ).

\subsubsection{Longitudinal Model Identification}

The goal for the longitudinal model identification was to identify the $2^{\text {nd }}$ order short-period model from flight test data:

$$
\left[\begin{array}{c}
\dot{\alpha} \\
\dot{q}
\end{array}\right]=A_{l g}\left[\begin{array}{c}
\alpha \\
q
\end{array}\right]+B_{l g} \delta_{E}
$$


Two sets of data - as shown in Figure 3-9 and 3-10 - were selected from recorded flight data - one for identification and another for a validation purpose. The data was sampled at $100 \mathrm{~Hz}$. It should be noted that the data had been pre-processed - the non-zero steady state value of $\alpha$ and $\delta_{E}$ were removed - before being used for identification process. Both data sets were associated with typical elevator doublet maneuver, which were able to sufficiently excite the longitudinal short-period mode.

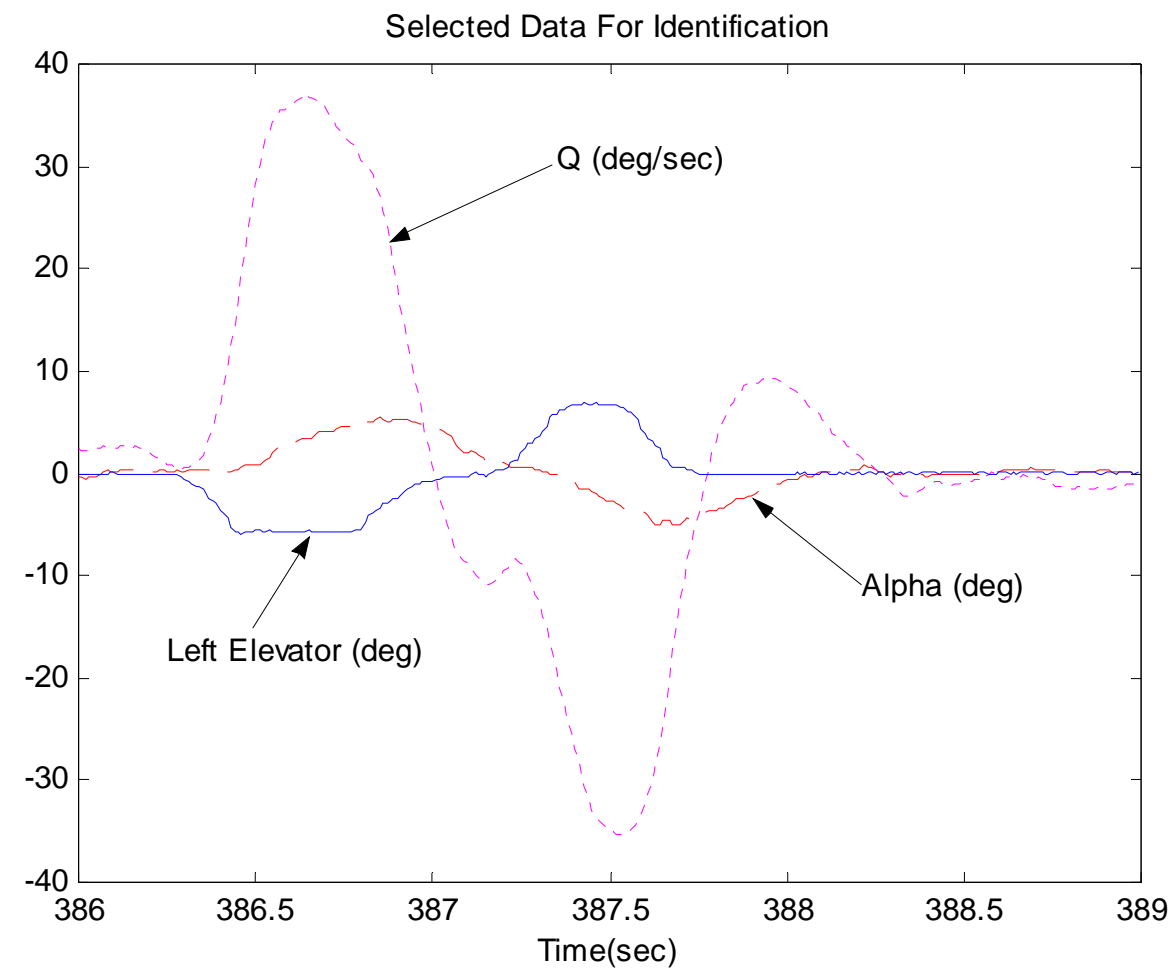

Figure 3-9 Data for Longitudinal Model Identification 


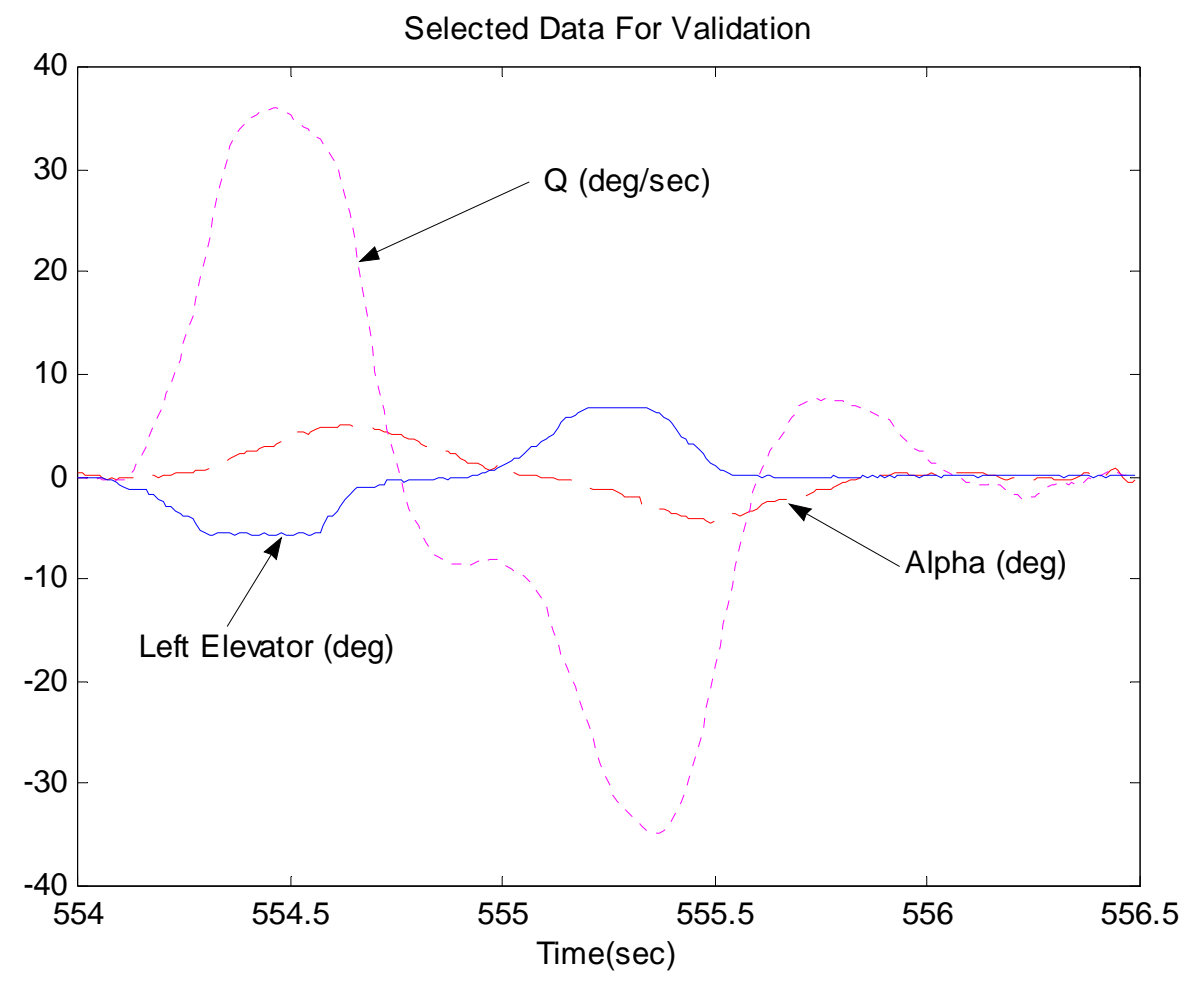

Figure 3-10 Data for Longitudinal Model Validation

With the BLS method, the longitudinal model was identified as:

$$
\begin{gathered}
{\left[\begin{array}{l}
\dot{\alpha} \\
\dot{q}
\end{array}\right]=\left[\begin{array}{ll}
-4.1172 & 0.7781 \\
-33.8836 & -3.5729
\end{array}\right]\left[\begin{array}{l}
\alpha \\
q
\end{array}\right]+\left[\begin{array}{c}
0.5435 \\
-39.0847
\end{array}\right] \delta_{e}} \\
q=\left[\begin{array}{ll}
0 & 1
\end{array}\right]\left[\begin{array}{l}
\alpha \\
q
\end{array}\right]+[0] \delta_{e}
\end{gathered}
$$

This continuous state-space model was then converted into a discrete model for simulation purposes:

$$
\begin{gathered}
{\left[\begin{array}{l}
\alpha(n+1) \\
q(n+1)
\end{array}\right]=\left[\begin{array}{ll}
0.9161 & 0.0144 \\
-0.6264 & 0.9261
\end{array}\right]\left[\begin{array}{l}
\alpha(n) \\
q(n)
\end{array}\right]+\left[\begin{array}{r}
0.0046 \\
-0.7566
\end{array}\right] \delta_{e}(n)} \\
q(n)=\left[\begin{array}{ll}
0 & 1
\end{array}\right]\left[\begin{array}{l}
\alpha(n) \\
q(n)
\end{array}\right]+[0] \delta_{e}(n)
\end{gathered}
$$

The sampling time for the discrete system was $T_{s}=0.02 \mathrm{sec}$

Figure 3-11 and 3-12 demonstrate the measured and computed responses for the pitch rate and angle of attack respectively. It shows that the identified model can reproduce the short-period dynamics with a satisfactory quality. 


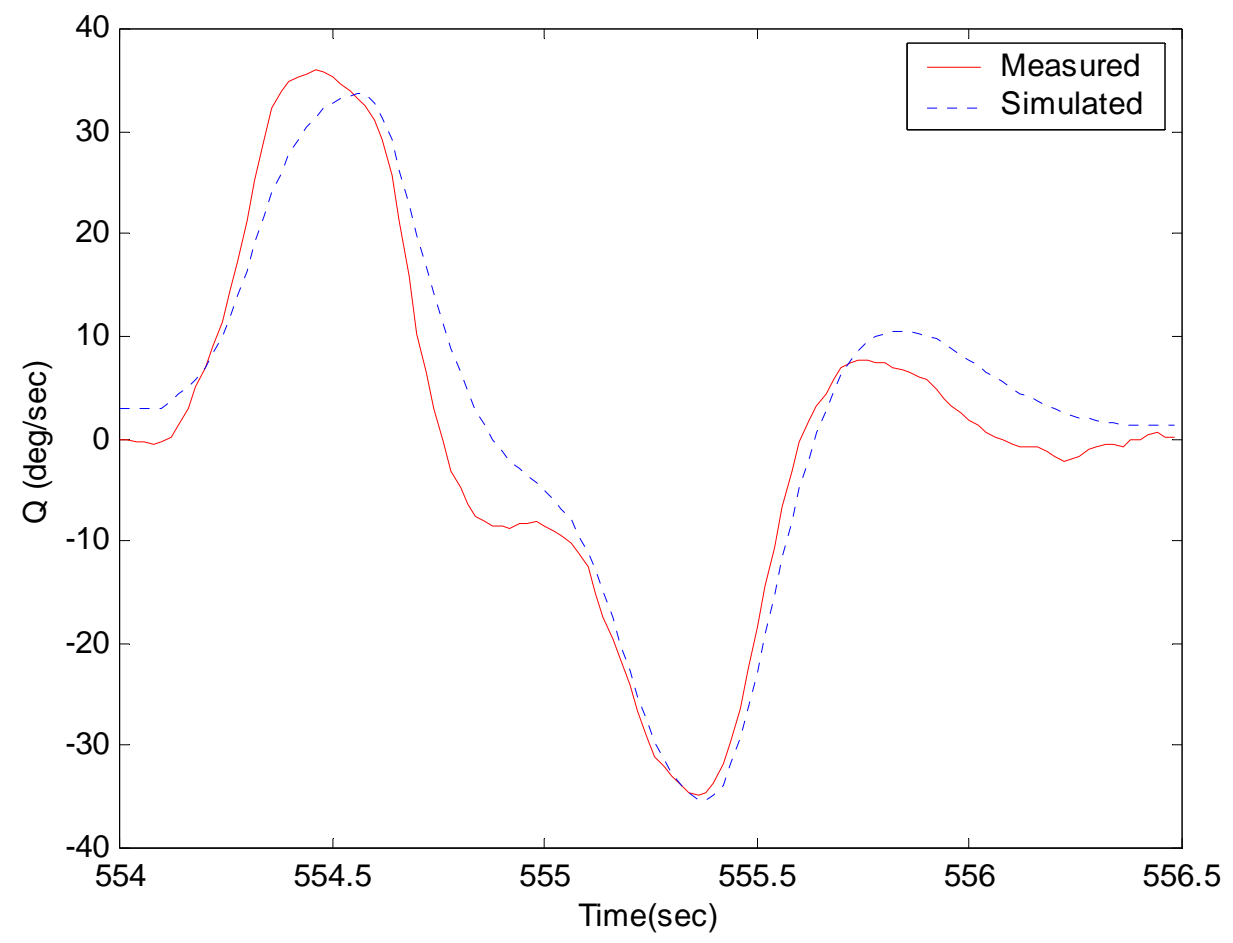

Figure 3-11 Model Validation-Measured and Simulated Pitch Rate

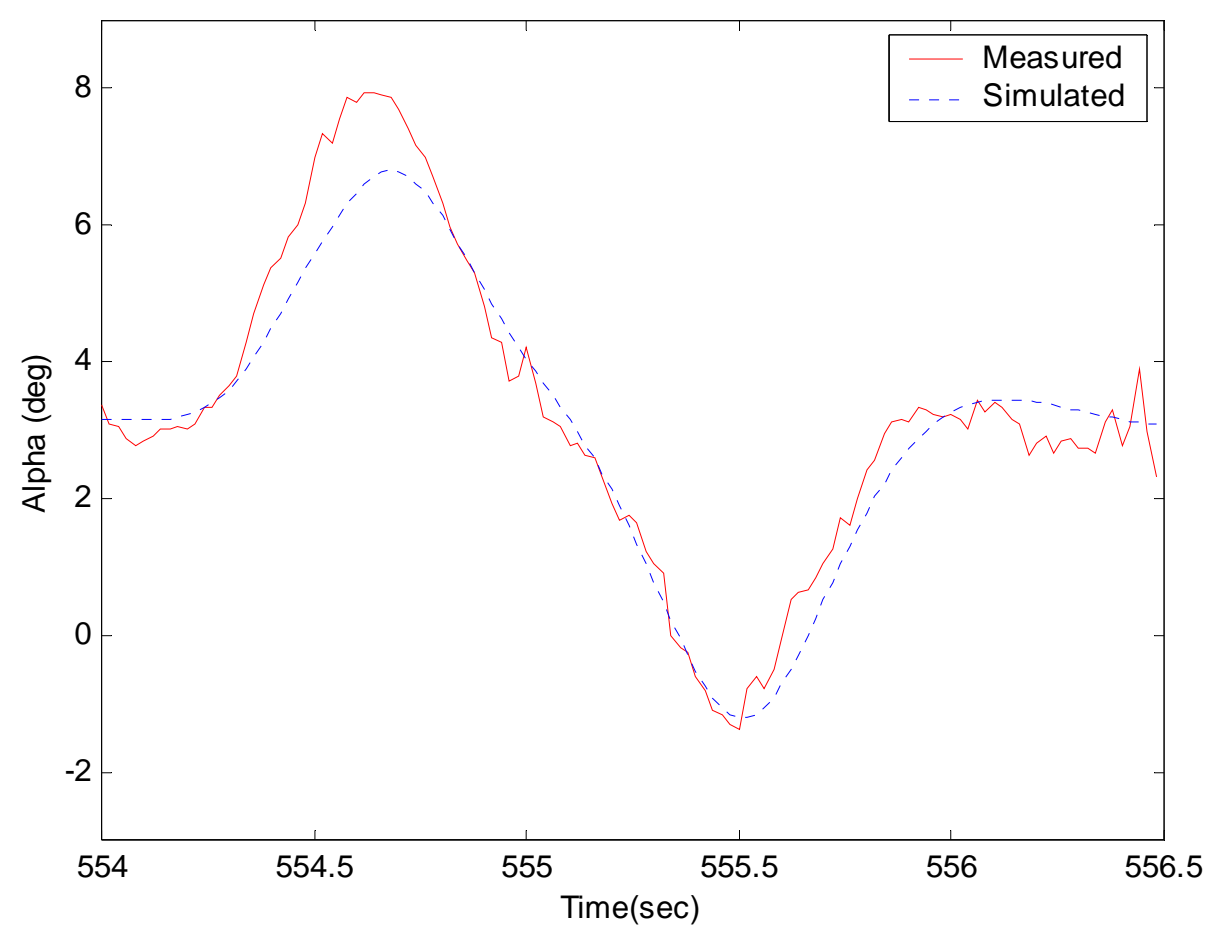

Figure 3-12 Model Validation-Measured and Simulated Angle-of-attack 


\subsubsection{Lateral-Directional Model Identification}

The task of lateral-directional model identification was to identify the following $3^{\text {rd }}$ order linear model from collected flight test data:

$$
\left[\begin{array}{c}
\dot{\beta} \\
\dot{p} \\
\dot{r}
\end{array}\right]=A_{l d}\left[\begin{array}{c}
\beta \\
p \\
r
\end{array}\right]+B_{l d}\left[\begin{array}{l}
\delta_{A} \\
\delta_{R}
\end{array}\right]
$$

Similar to longitudinal identification, two sets of data, as shown in Figures 3-13 and 3-14, were selected from flight testing data collection - one for identification and the other for validation purposes. The flight data was sampled at a rate of $100 \mathrm{~Hz}$. Unlike the longitudinal situation, there was no need to conduct data pre-processing since all the steady-state values of the sampled data were found to be very close to zero. Both data sets are representative of a typical aileron/rudder doublet combination, which includes an aileron doublet followed by a rudder doublet maneuver immediately after. This maneuver was found to provide sufficient excitation for both the dutch-roll mode and the roll response; therefore it was ideal for lateral-directional identification purposes.
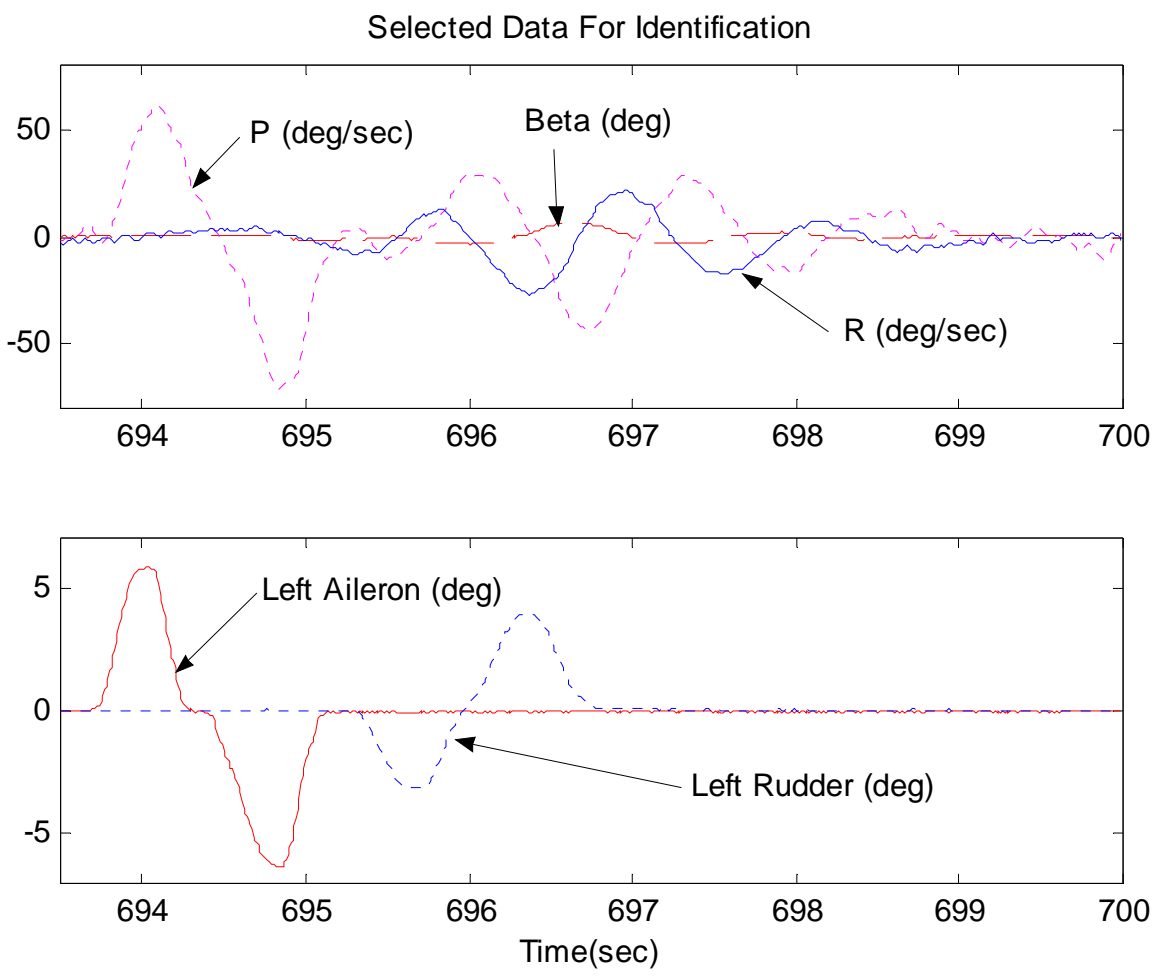

Figure 3-13 Data for Lateral-Directional Model Identification 

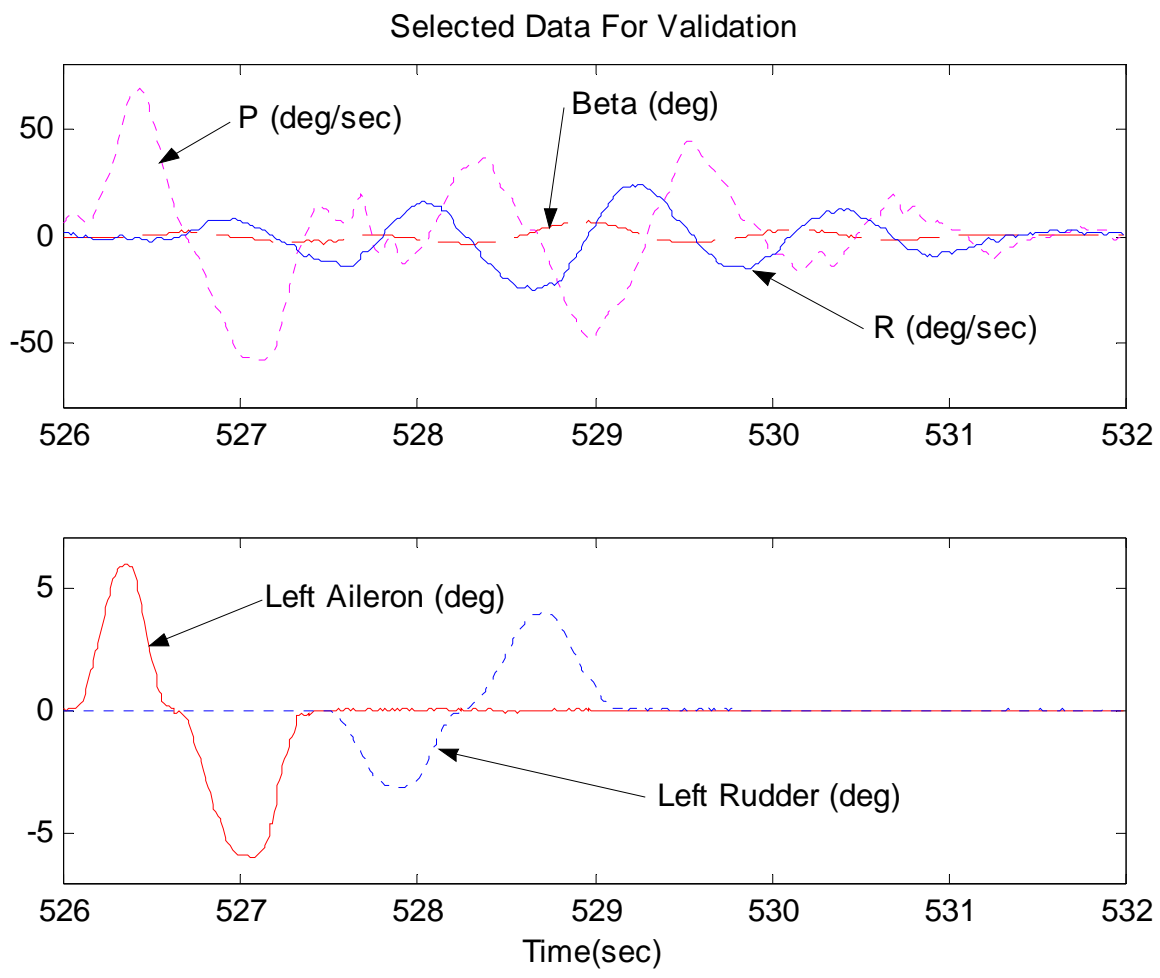

Figure 3-14 Data for Lateral-Directional Model Validation

Again, using a BLS method, the following lateral-directional model was identified as:

$$
\begin{gathered}
{\left[\begin{array}{c}
\dot{\beta} \\
\dot{p} \\
\dot{r}
\end{array}\right]=\left[\begin{array}{ccc}
0.4299 & 0.0938 & -1.0299 \\
-67.3341 & -7.9485 & 5.6402 \\
20.5333 & -0.6553 & -1.9955
\end{array}\right]\left[\begin{array}{l}
\beta \\
p \\
r
\end{array}\right]+\left[\begin{array}{cc}
0.2724 & -0.7713 \\
-101.8446 & 33.4738 \\
-6.2609 & -24.3627
\end{array}\right]\left[\begin{array}{l}
\delta_{a} \\
\delta_{r}
\end{array}\right]} \\
{\left[\begin{array}{l}
p \\
r
\end{array}\right]=\left[\begin{array}{lll}
0 & 1 & 0 \\
0 & 0 & 1
\end{array}\right]\left[\begin{array}{l}
\beta \\
p \\
r
\end{array}\right]+\left[\begin{array}{ll}
0 & 0 \\
0 & 0
\end{array}\right]\left[\begin{array}{l}
\delta_{a} \\
\delta_{r}
\end{array}\right]}
\end{gathered}
$$

As for the longitudinal model, this model was then converted into a discrete model with sampling time $T_{s}=0.02 \mathrm{sec}$

$$
\begin{aligned}
& {\left[\begin{array}{l}
\beta(n+1) \\
p(n+1) \\
r(n+1)
\end{array}\right]=\left[\begin{array}{rrr}
1.0032 & 0.0019 & -0.0201 \\
-1.2262 & 0.8512 & 0.1150 \\
0.4118 & -0.0115 & 0.9560
\end{array}\right]\left[\begin{array}{l}
\beta(n) \\
p(n) \\
r(n)
\end{array}\right]} \\
& +\left[\begin{array}{rr}
0.0048 & -0.0099 \\
-1.8926 & 0.6005 \\
-0.1092 & -0.4841
\end{array}\right]\left[\begin{array}{l}
\delta_{a}(n) \\
\delta_{r}(n)
\end{array}\right]
\end{aligned}
$$




$$
\left[\begin{array}{l}
p(n) \\
r(n)
\end{array}\right]=\left[\begin{array}{lll}
0 & 1 & 0 \\
0 & 0 & 1
\end{array}\right]\left[\begin{array}{l}
\beta(n) \\
p(n) \\
r(n)
\end{array}\right]+\left[\begin{array}{ll}
0 & 0 \\
0 & 0
\end{array}\right]\left[\begin{array}{l}
\delta_{a}(n) \\
\delta_{r}(n)
\end{array}\right]
$$

Figure 3-15 through 3-17 demonstrate the validation of the identified linear lateral-directional model. The measured and computed outputs for sideslip angle, roll and yaw rate are shown. From the validation results, it can be concluded that the identified model can re-produce the lateral-directional dynamics within a desirable level of accuracy.

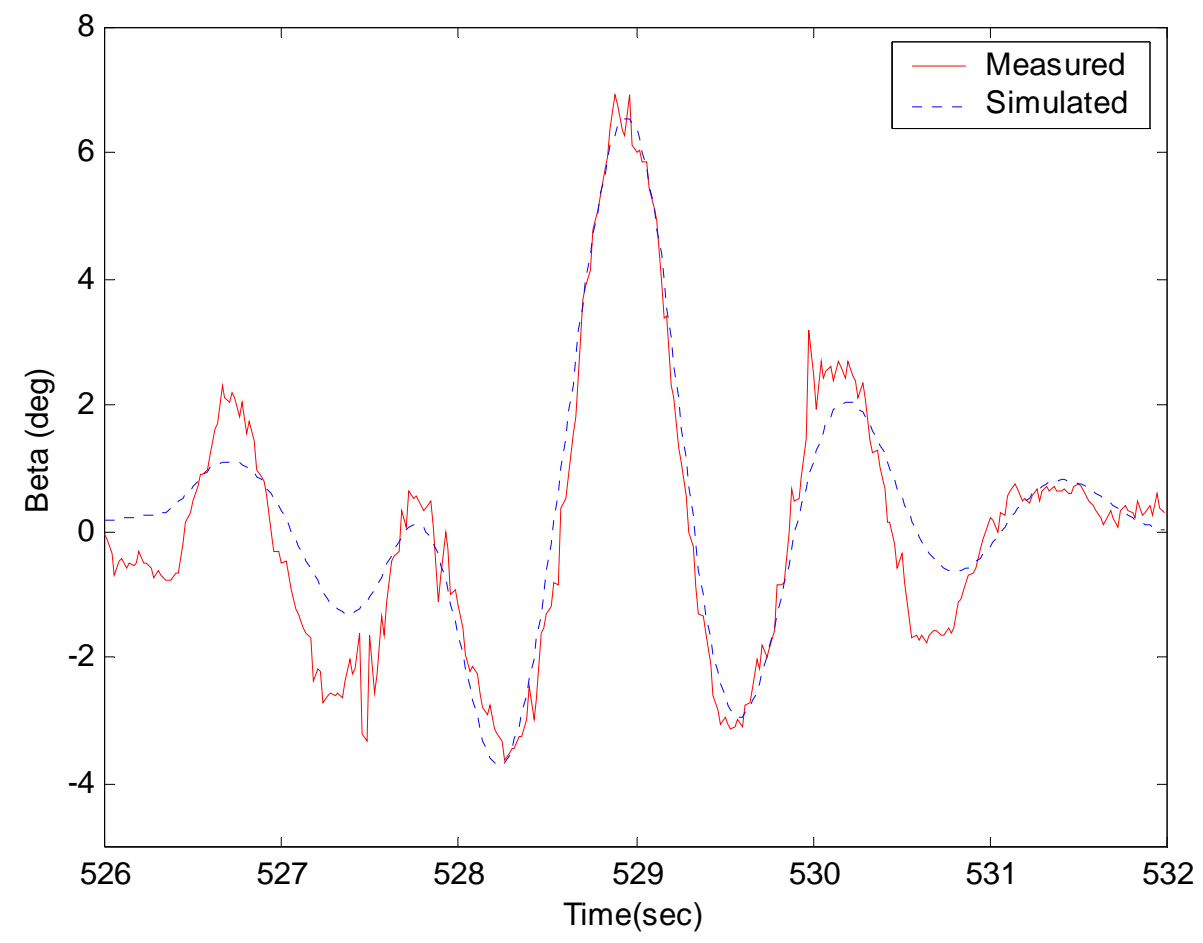

Figure 3-15 Model Validation - Measured and Simulated Sideslip Angle 


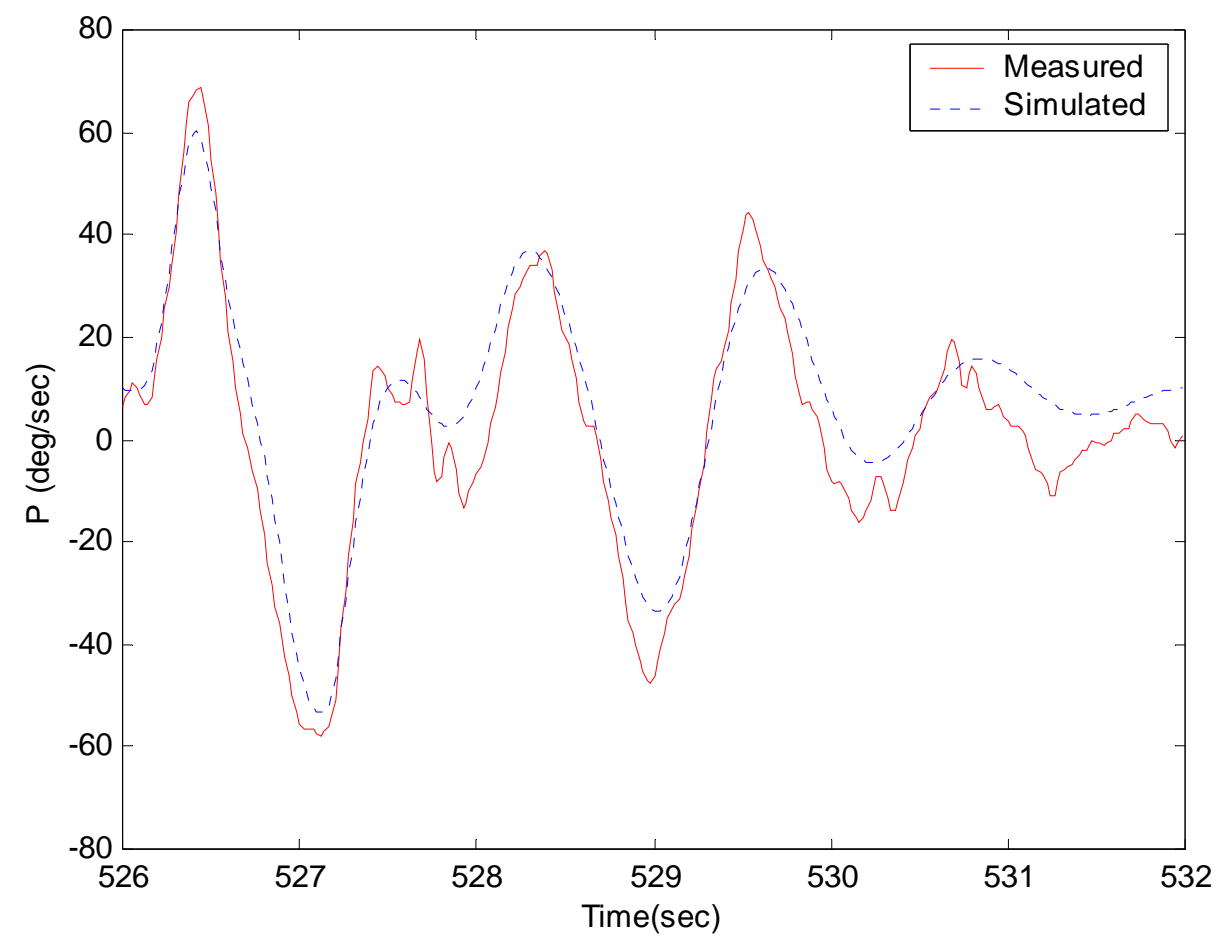

Figure 3-16 Model Validation-Measured and Simulated Roll Rate

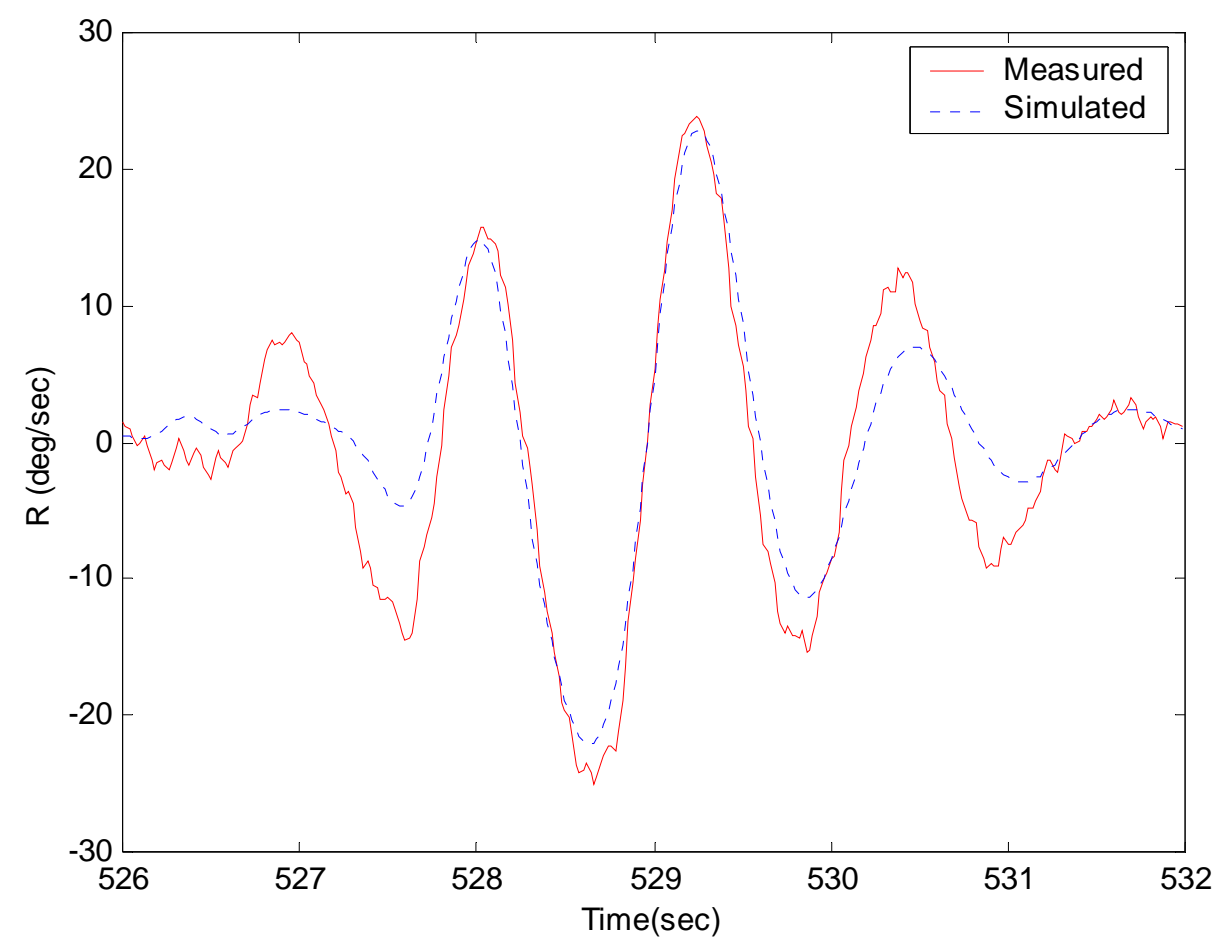

Figure 3-17 Model Validation - Measured and Simulated Yaw Rate 


\subsubsection{Actuator Model Identification}

The actuators used in WVU research UAVs to drive all the aircraft control surfaces were digital servos made by JR Corp. (Figure 3-18). A model of the actuator itself was needed for both controller design and flight control system simulation.

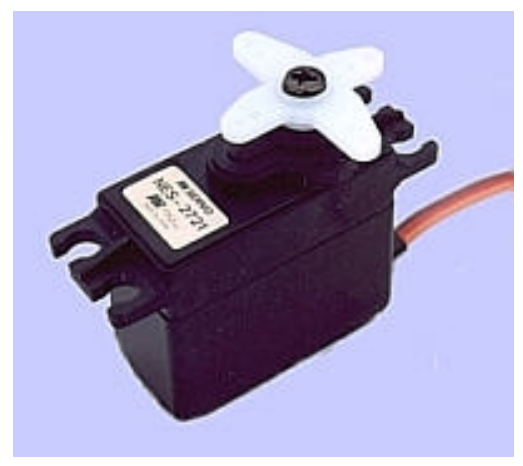

Figure 3-18 R/C Servo

The importance of a good actuator model relies in the fact that the bandwidth of the whole flight control system is mainly dominated by the actuator's bandwidth. Since the command to the actuator's position was issued from the on-board computer through the controller board, the actuator model was thus defined as the transfer function from the digital command from the computer to the actuator's actual position. This definition is slightly different from the 'conventional' actuator model, in that the interface between the computer and the actuator (featured by a pure time delay in this case, as will be seen shortly) was also included in the model; this facilitates the analysis and design of the control system.

To conduct the actuator model identification, a ground-test experiment was performed by applying step input from the on-board computer to the actuator, and the actuator's position response data was read back to the computer via the data acquisition card. Both the command and data sampling rate was set at $50 \mathrm{~Hz}$. The experiment was performed for all of the 6 major actuators installed on the aircraft model (left/right elevators, left/right ailerons and rudders) and identification was attempted for all test data collected from the six actuators. Figure 3-19 represents one typical actuator test used for identification purposes. 


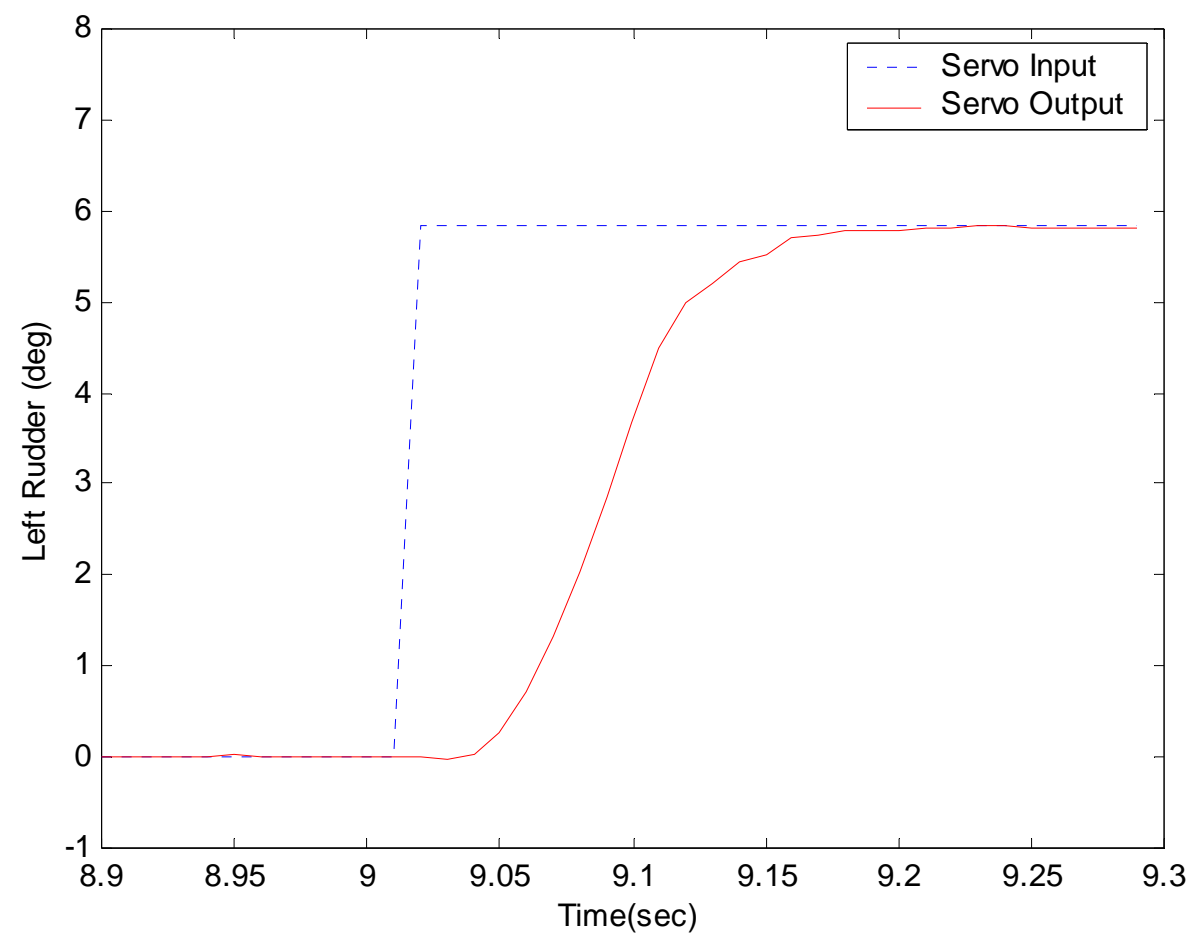

Figure 3-19 Data from Actuator Identification

It was found that the actuator model was best described by the following transfer function:

$$
G_{a}=\frac{1}{1+\tau_{a} s} e^{-\tau_{d} s}
$$

where $\tau_{d}$ is the pure time delay and takes a fixed value of $0.02 \mathrm{sec}$ for all six actuators. $\tau_{a}$ is the actuator time constant taking different values for each actuator, as listed in Table 3-2:

\begin{tabular}{|l|l|l|l|l|l|}
\hline Left elevator & Right elevator & Left rudder & Right rudder & Left aileron & Right aileron \\
\hline 0.0375 & 0.0294 & 0.0294 & 0.0313 & 0.0424 & 0.0391 \\
\hline
\end{tabular}

Table 3-2 Actuator time constant

A value of 0.0424 , which was the longest (and thus most conservative) among the values in the above table, was selected as the time constant for the actuator model. Figure 3-20 shows the measured and simulated step response of the identified actuator model. 


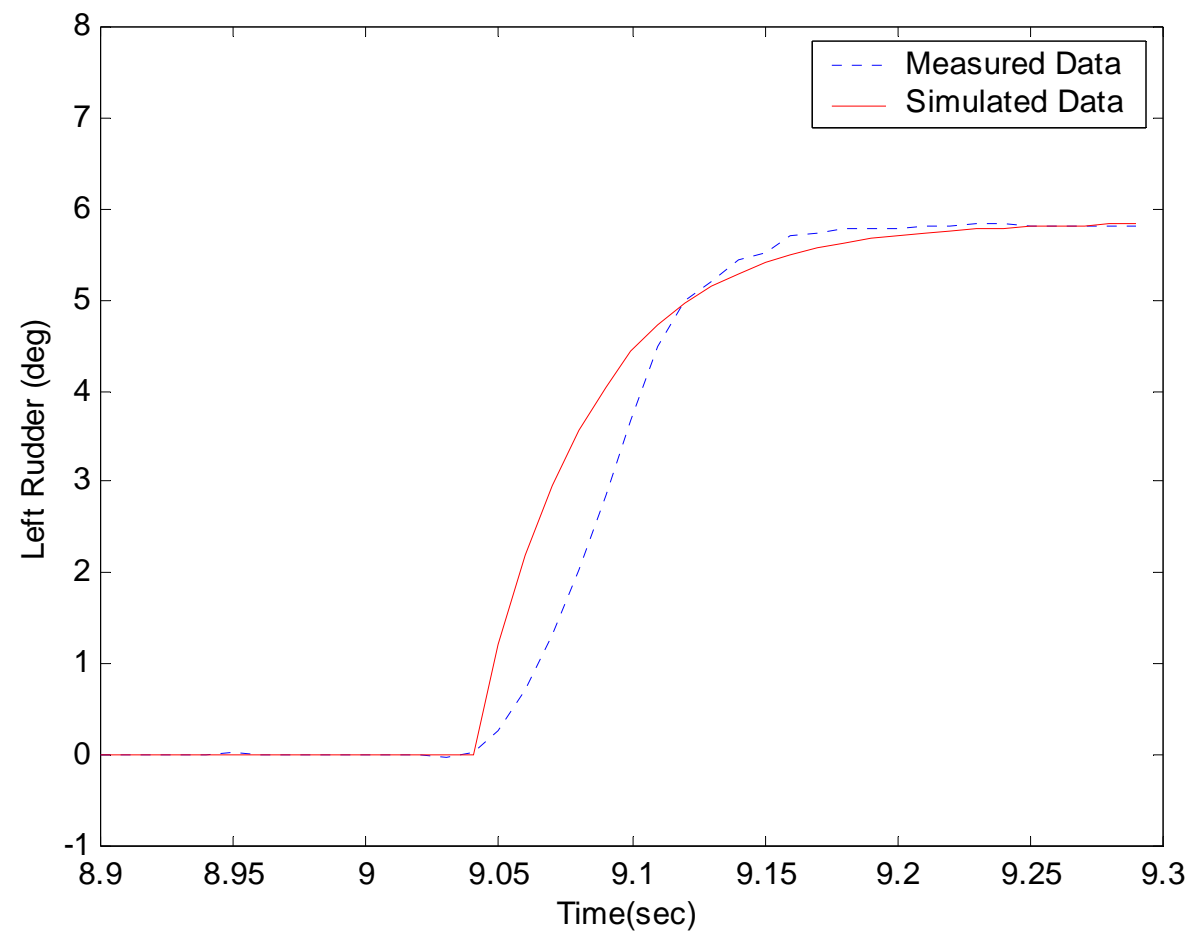

Figure 3-20 Measured and Simulated Actuator Step-response

Since the full actuator models were associated with the aerodynamic force/torque load, the final actuator model was validated/verified from actual flight test data. Sample flight data shown in Figure 3-21 was collected from flight test session Oct.16 ${ }^{\text {th }} 2003$. The flight data represents the input-output relationship of the left rudder actuator. 


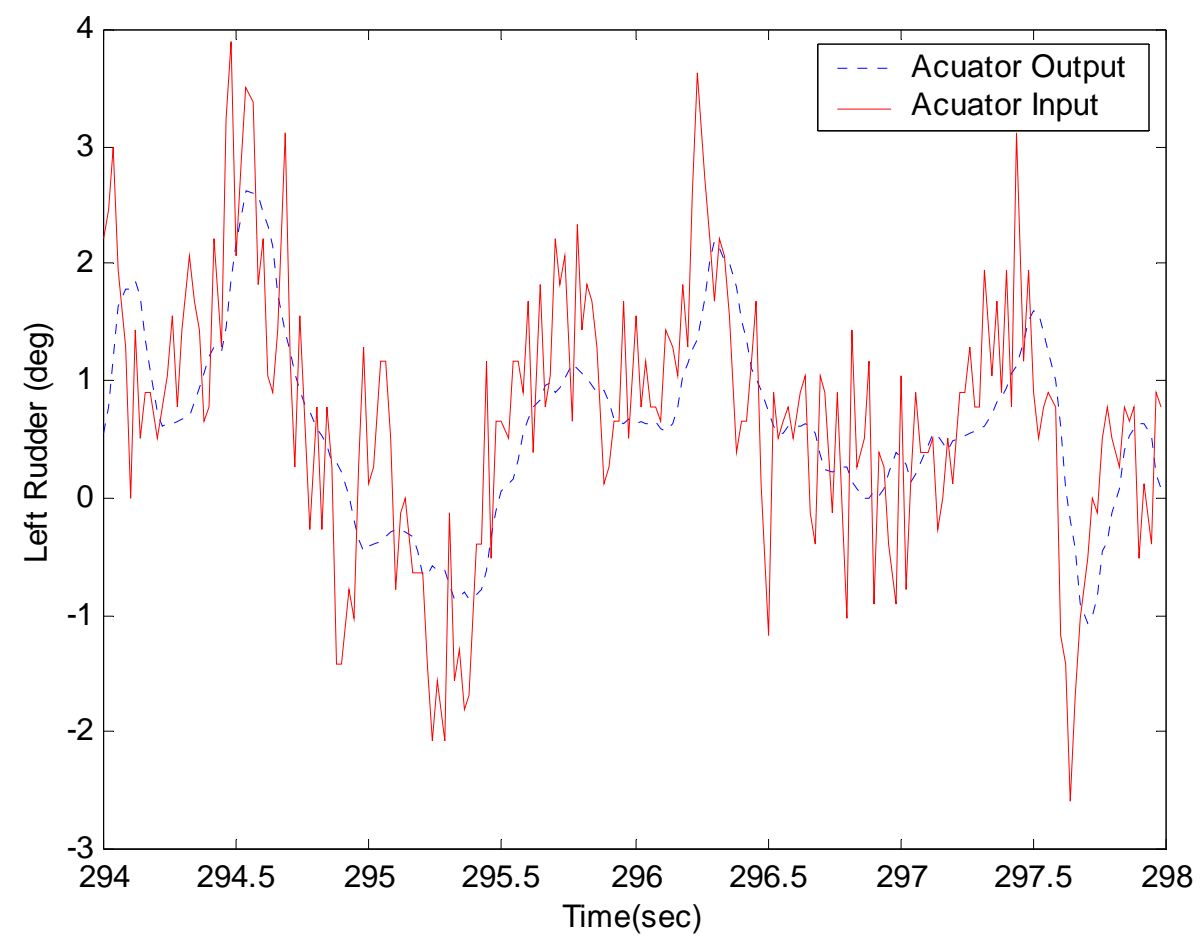

Figure 3-21 In-flight Actuator's Response

It is clearly shown that the bandwidth of the actuator limited the response of the flightcontrol system. The actuator worked as a low pass filter (with delay) and smoothed out the noisy control command caused by the rate-sensor feedback but still maintain enough speed to control the aircraft.

With the estimated actuator model:

$$
G_{a}=\frac{1}{1+0.0424 s} e^{-0.02 s}
$$

A simulation test was performed to simulate the actuator response. Figure 3-22 shows the comparison of the measured (in-flight) and computed actuator's response with the same controller command shown in Figure 3-21. 


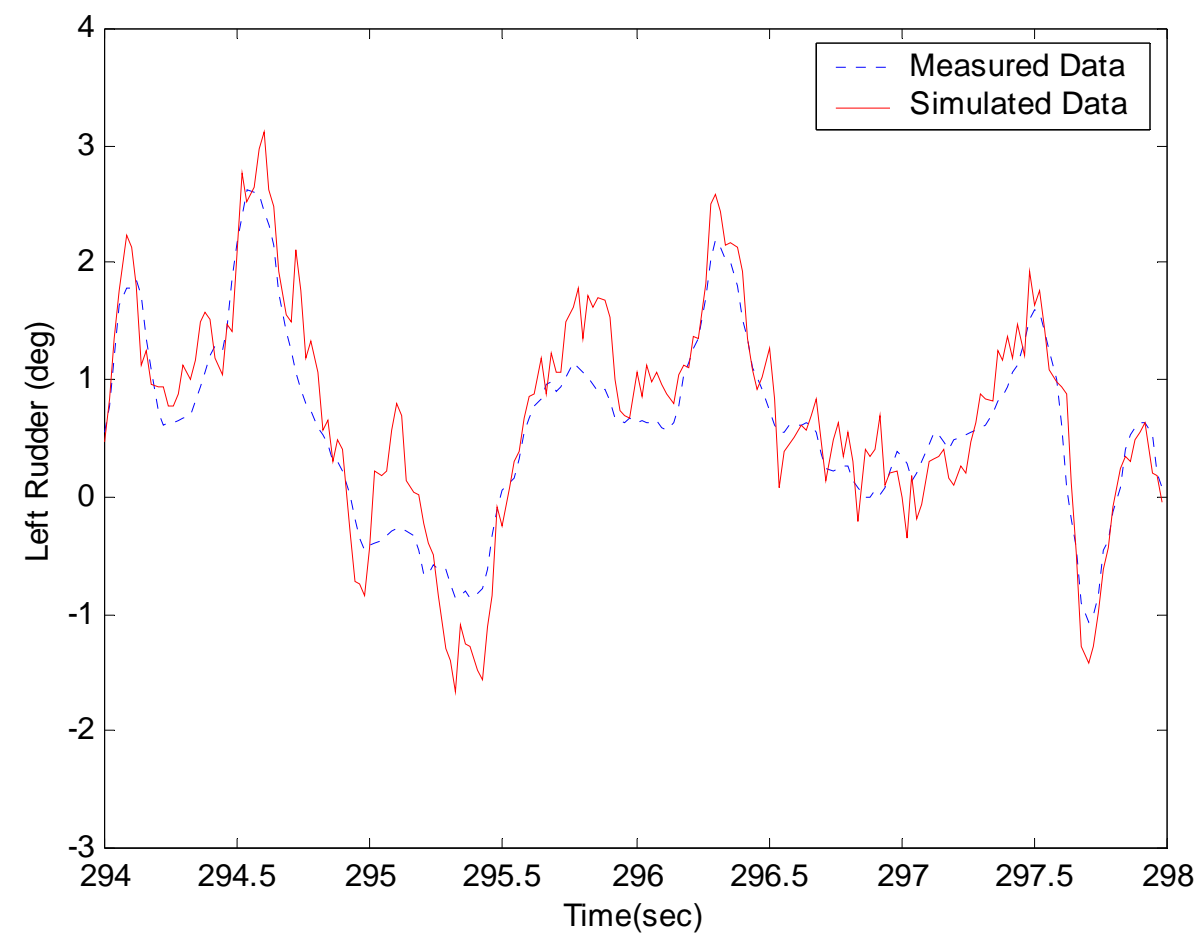

Figure 3-22 Model Validation - Measured and Simulated Actuator In-flight Response

It can be concluded that the identified actuator model can approximate the actual servo response within a desirable level of accuracy.

\section{4 - Linear Controller Design}

A linear feedback controller was designed as a baseline system to provide the reference point for analysis. Designed at the nominal flight condition, the linear controller has the capability of stabilizing the aircraft during a nominal flight condition (without failure) and provides a "basis" for the NN controller during actuator failure. Angular rate readings obtained from the Inertial Measurement Unit (IMU) were used for feedback. A more detailed explanation of the on-board hardware/software flight control package will be provided in Chapters 4 and 5 .

The linear controller can be considered as a Stability Augmentation System (SAS). The SAS typically uses sensors to measure the body-axes angular rates of the vehicle, feeding back a processed version of the signals to the servomechanisms that drive the aerodynamics control surfaces. In this way, an aerodynamic moment 
proportional to the angular velocity and its derivatives can be generated to produce a damping effect on the motion. Detailed description and design methods can be found in [44]. Stability augmentation systems are conventionally designed separately for the longitudinal dynamics and lateral-directional dynamics. This is made possible by decoupling of aircraft dynamics in most flight conditions (without actuator failure). The design process of these two sub-controllers will be presented below.

\subsubsection{Longitudinal Control Parameter Design}

The purpose of a longitudinal SAS is to provide a desirable natural frequency and damping for the short-period mode. The pitch rate feedback gain is preferred to be as large as possible to compensate for wind gust disturbances while still maintaining a reasonable stability margin and short-period damping ratio.
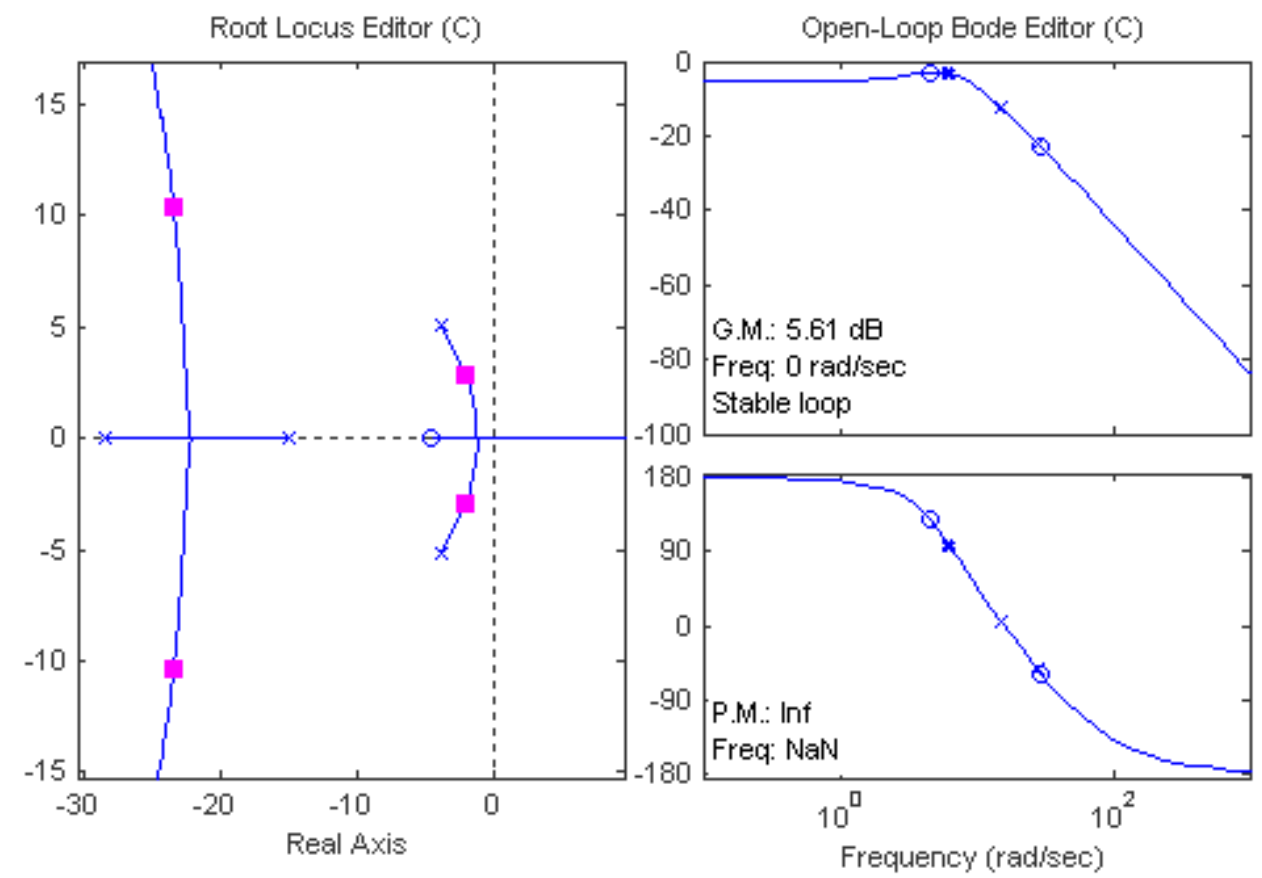

Figure 3-23 Root-locus (Longitudinal Dynamics)

Using a root-locus based design as shown above in Figure 3-23, the pitch rate feedback gain was selected to be:

$$
K_{q}=0.12
$$

This gives a satisfactory damping ratio of 0.592 and a natural frequency of $3.56 \mathrm{rad} / \mathrm{sec}$ 


\subsubsection{Lateral-Directional Control Parameter Design}

The lateral-directional control design was basically a Multi-Input, Multi-Output (MIMO) controller design. This requires a detailed understanding of the control system in order to proceed with a design phase. Once a control gain for a specific feedback is designed, the corresponding feedback loop is closed and thus forming a new closed loop system on which the next feedback gain design is based upon.

\section{Yaw rate feedback gain/washout filter constant design}

The purpose of the stability augmentation yaw-rate feedback was to use the rudder control surface to generate a yawing moment which opposes any yaw rate associated with the dutch roll. The design objective was to achieve the dutch roll damping ratio a reasonable value by designing yaw rate feedback gain, $K_{r}$, while choosing a reasonable washout filter constant. Note that the uncompensated dutch roll damping ratio was 0.192 . The assumption is that the washout filter has the following format:

$$
G_{w}(s)=\frac{s}{s+\omega_{0}}
$$

Through an iterative process a $K_{r}$ value was selected, which maximizes the dutch-roll damping ratio with different $\omega_{0}$, the best roll feedback gain value obtained was

$$
K_{r}=0.16
$$

where $\omega_{0}=1.8$. With this design, the dutch roll damping ratio was 0.7 and the natural frequency was $7.47 \mathrm{rad} / \mathrm{sec}$.

\section{Roll angle/rate feedback gains design}

To achieve a desirable gust disturbance attenuation and fast roll rate stabilization, the objective of the roll rate feedback gains design was to find a gain as large as possible, while maintaining a reasonable stability margin and damping ratio. In this design, the yaw rate feedback loop was then closed. The designed roll rate feedback gain was:

$$
K_{p}=0.04
$$

leading to a damping ratio of 0.86 , 
Therefore, the final set of feedback gains for the linear controller design were found to be:

$$
K \_q=0.12 ; K \_p=0.04 ; K \_r=0.16
$$

with a washout filter on the yaw rate control channel defined as:

$$
G_{w o}=\frac{s}{s+1.8}
$$

The linear controller was designed to stabilize the aircraft at nominal flight condition and provided a baseline controller performance for the NN based AFA controller design.

\subsubsection{Linear Controller Validation}

To validate the performance of the designed linear controller, a set of flight tests were performed. During the flight testing, the pilot may use the controller switch to activate the on-board controller. In this way, the pilot can perform a maneuver (i.e. doublets) with individual control channel (elevator, aileron or rudder) to excite the aircraft dynamics and turn on the linear controller right afterwards. The linear controller would then send control commands to stabilize the aircraft. Figure 3-24 shows the aircraft's longitudinal response after an elevator maneuver. Figures 3-25 and 3-26 represent the aircraft's lateral-directional response after the aileron and rudder maneuvers were performed. 

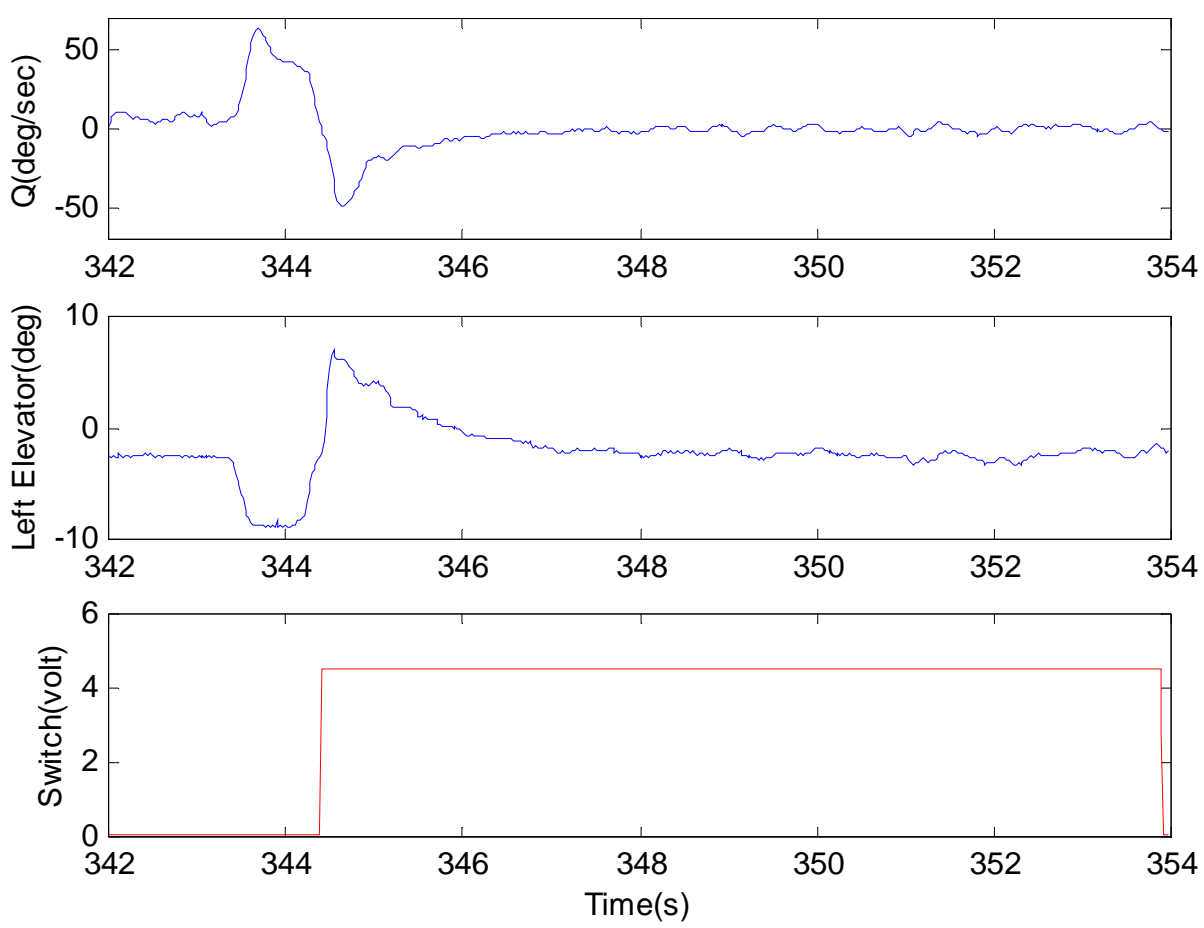

Figure 3-24 Linear Controller Performance after Elevator Maneuver
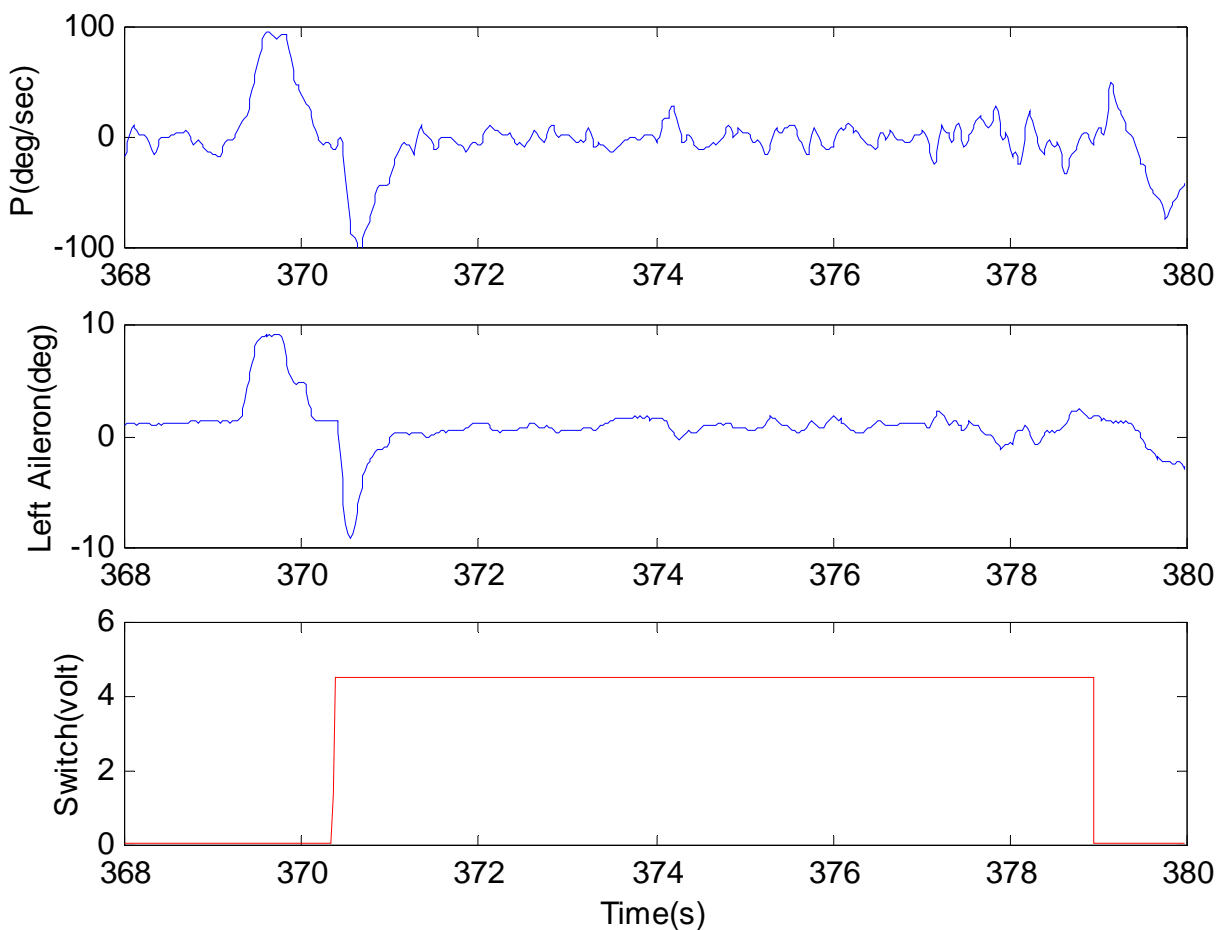

Figure 3-25 Linear Controller Performance after Aileron Maneuver 

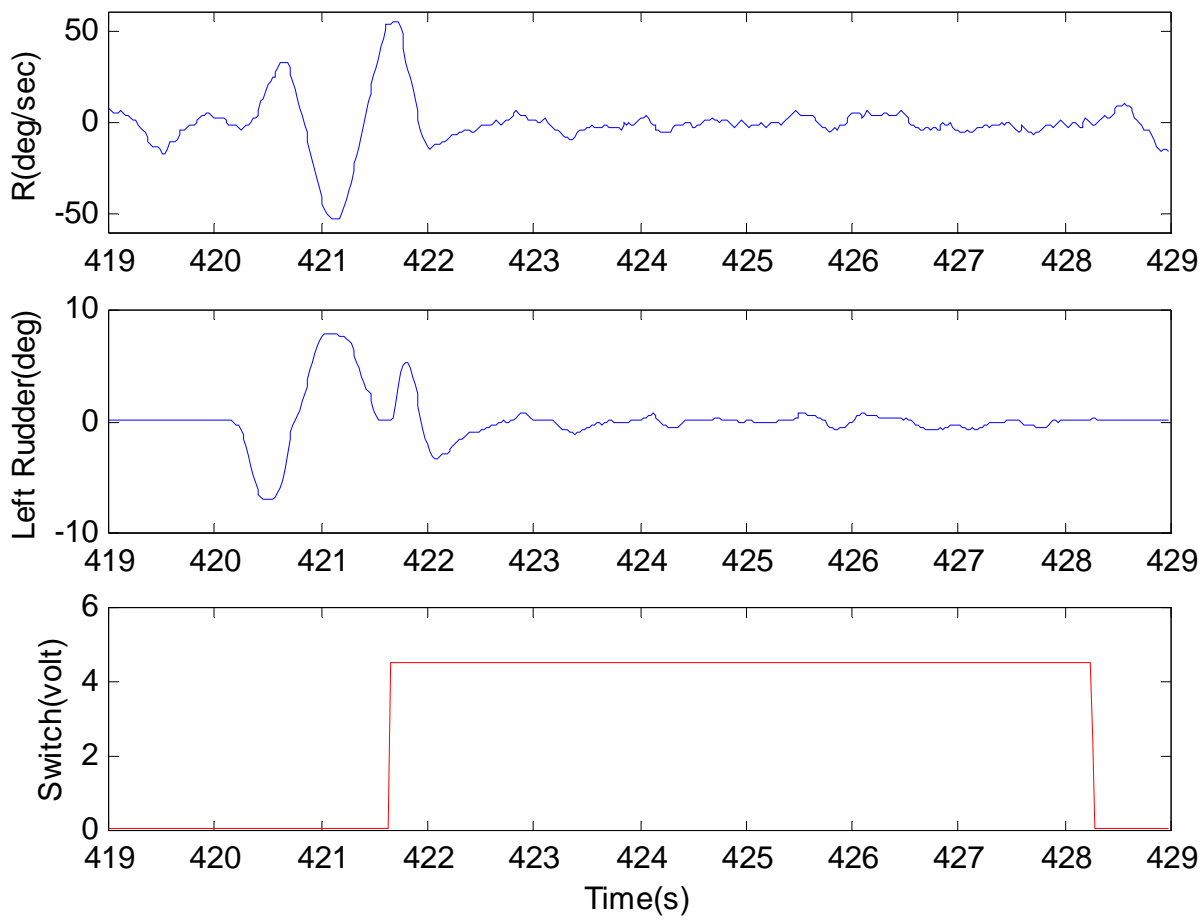

Figure 3-26 Linear Controller Performance after Rudder Maneuver

From the above flight testing results, it can be concluded that the designed linear controller can effectively stabilize the aircraft at the nominal flight condition (without failure).

As a small UAV with only $6.5 \mathrm{ft}$ wingspan, wind gusts can be a major source of disturbance, especially on the roll rate channel (Figure 3-25). This effect can only be decreased by increasing the feedback control gains. Flight test with a set of higher gains were performed; however, the aircraft closed-loop stability was compromised by these high values of the control gains. As expected, wind gusts also negatively affect the NN learning, which will be discussed in Chapter 6 .

\section{5 - Aileron Failure AFA Controller Design}

In the scenario of aileron failure, the right aileron is locked at the trim position while the left aileron remained functional. Flight tests were completed with this 
configuration without the on-board controller involved. During the flight testing, the right aileron was locked at trim instantly once the controller switch triggered. The pilot can use the remaining left aileron to perform aileron doublets maneuvers and the flight data was recorded and compared with the nominal flight conditions. Selected flight testing data are shown in Figure 3-27 and 3-28. It shows that during the aileron failure (right aileron locked at trim), two adverse effects typically developed:

1. Roll control authority deteriorates. This is quite straightforward since the effective aileron area has been decreased into half. Flight data shown in Figure 3-27 is a comparison of two different flight configurations. The solid line shows the roll rate response after a \pm 7 degree aileron doublet with right aileron failure and the dot line shows the response of the same maneuver without aileron failure. The roll rate response under failure is only about half of the nominal flight condition.

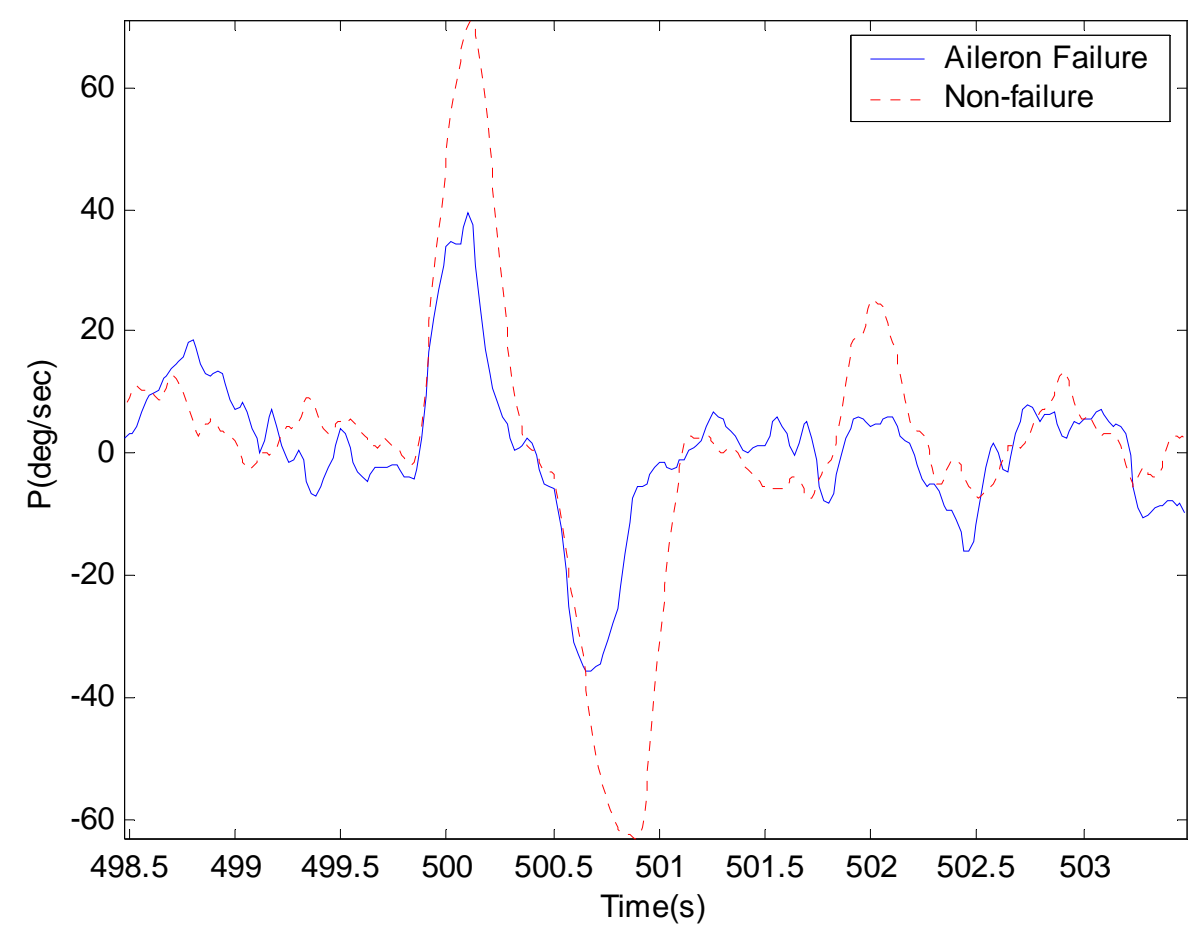

Figure 3-27 Aileron Failure Response - P channel

2. Unsymmetrical aileron deflection caused a mild pitching moment due to the small moment arm between the aircraft C.G. and the point of application of the force - associated with the deflection of the 'healthy' 
aileron - generation the roll. This effect can be observed in Figure 3-28. With the right aileron locked at trim position, the left aileron doublet caused a small motion in longitudinal channels.
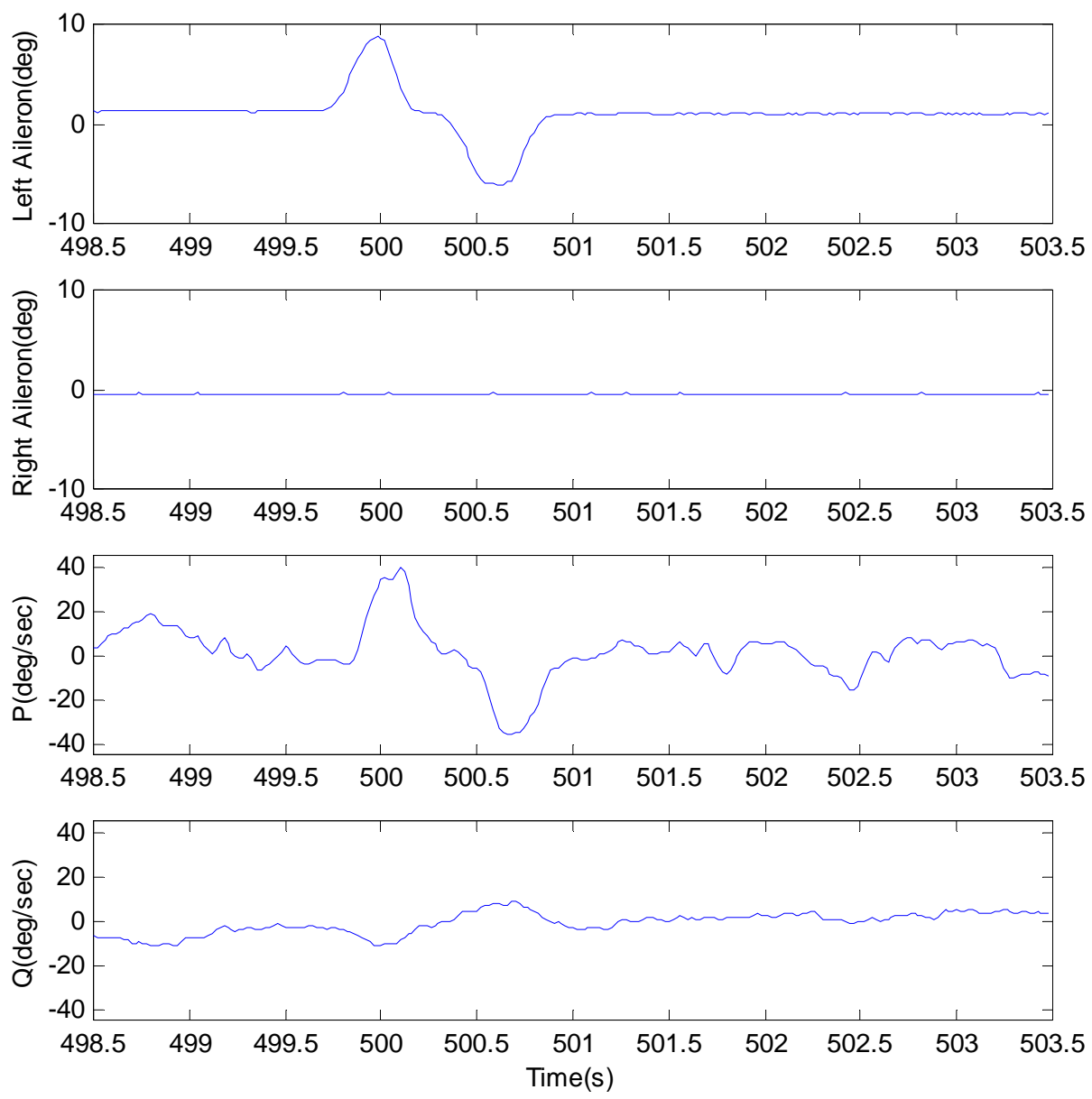

Figure 3-28 Aileron Failure Response- Coupling

Considering the size and position of ailerons on the YF-22 research UAV, the coupling of the longitudinal and lateral dynamics caused by aileron failure is - as expected - very minor and can be fully compensated for by the linear longitudinal controller. This is not the case, however, for the elevator failure scenario, which will be described next in Section 3.6.

The major effect from the aileron failure would be the aircraft's performance degradation caused by a decreased area of effective aileron surface. To evaluate the performance of the linear controller under a failed condition, different flight tests were 
performed with flight test data shown in Figures 3-29 and 3-30. Since the aileron failure was designed to be a locked control surface at a trim position, the effect of failure could not be shown in a straight and level flight condition since there is no difference between failure and non-failure conditions. The flight tests were then designed to let the controller follow a sine-wave pattern on the roll rate. The tracking accuracy was not important since the controller had been designed to stabilize the aircraft instead of being a tracking controller. With this configuration, the aircraft could continue the maneuvers once the controller switch is engaged and the effect of failure can be fully excited. This configuration could also help the on-line $\mathrm{NN}$ to learn the aircraft dynamics.

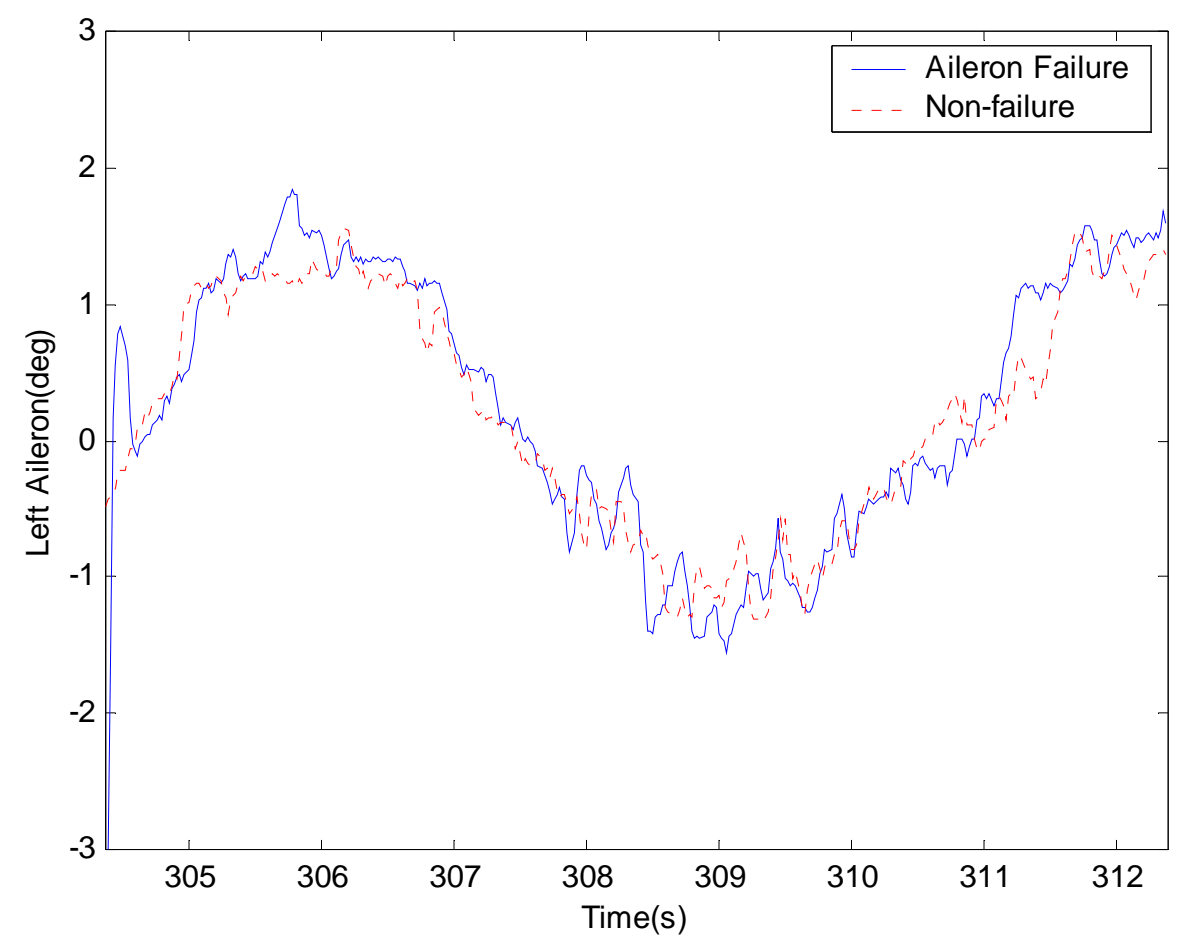

Figure 3-29 Linear Controller with/without Failure - Left Aileron 


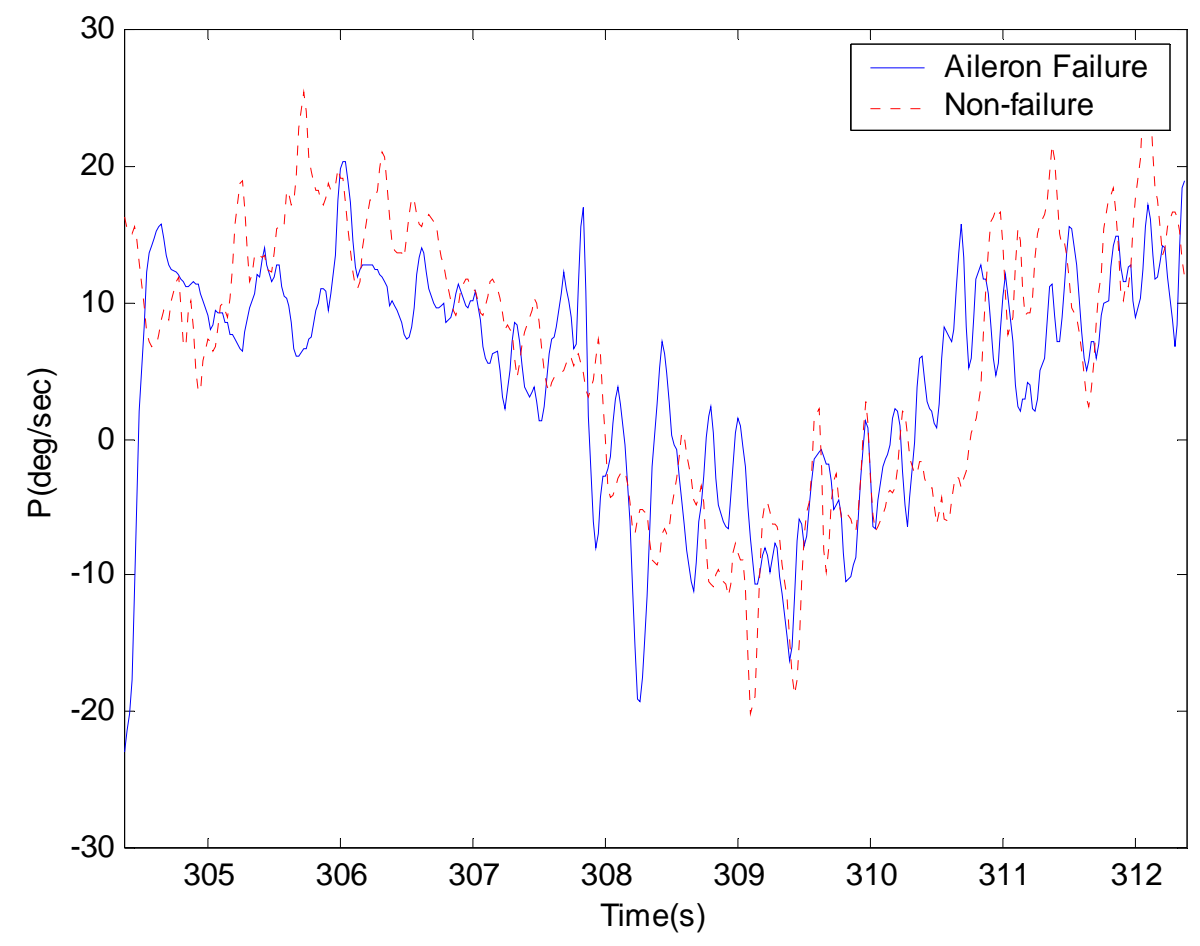

Figure 3-30 Linear Controller with/without Failure - P channel

In the case of linear controller with aileron failure, the feedback gain is fixed and will not accommodate for the failure. However, since the loss of half aileron area increased the tracking error, the command input on the ailerons would actually be increased by only a small amount. This effect can be noticed back in Figure 3-29, where the solid line shows the left aileron deflection with the existence of the right aileron failure and the dot line shows the same signal without failure. Even with this slight compensation effect, the response of the roll rate is still noticeably less effective than the condition without failure (shown in Figure 3-30, the signal is noisy because of wind gusts), which indicates the degradation of handling quality. The AFA controller was designed to learn from the failure and increase the feedback controller gain of the roll rate to compensate for the loss of the right aileron. The design of the aileron failure AFA controller is shown in Figure 3-31. 


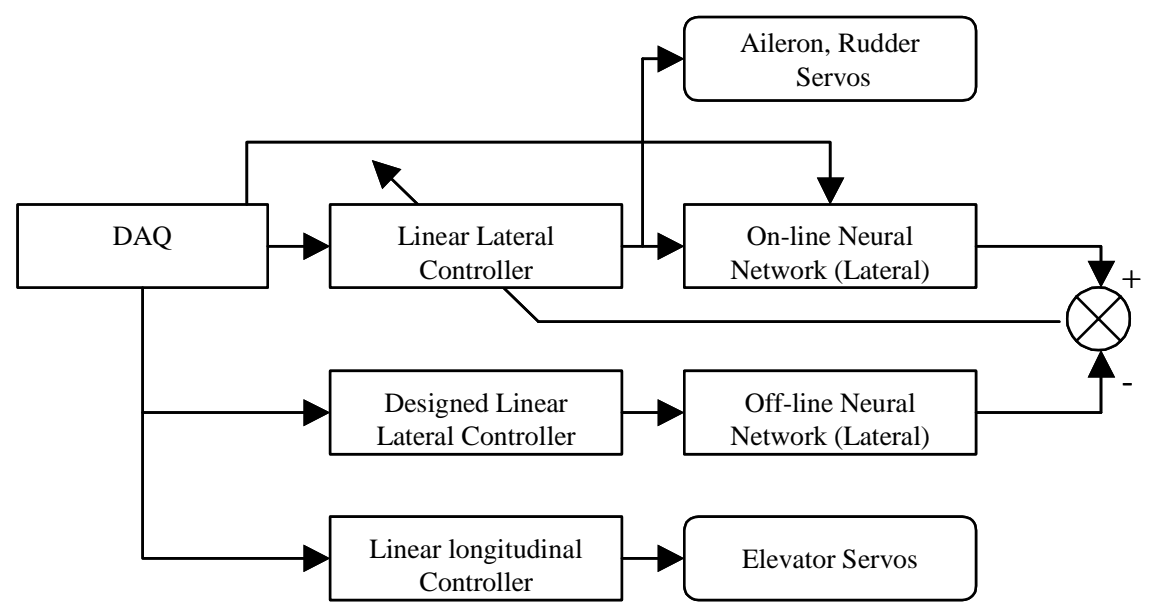

Figure 3-31 Aileron Failure AFA Controller

The on-line learning NN (described in Section 3-2) learns and approximates the lateraldirectional dynamics of the aircraft. The estimation of the $\mathrm{NN}$ is compared with the offline NN's outputs to calculate the modeling error. The linear controller feedback gains are then updated according to this modeling error to compensate for the failure condition. To decrease the negative effect of the wind gust turbulence and measurement noise, the gain updating procedure has been smoothed by using the difference of the standard deviation of the $\mathrm{NN}$ estimations of the last 50 time steps (one second total) instead of using them directly. The gain tuning procedure can be described as:

$$
\begin{aligned}
& K_{p}(n+1)=K_{p}(n)+l_{r} \times\left(\operatorname{Std}\left(P_{N N_{\text {off-line }}}(n-50) \ldots P_{N N_{\text {off-line }}}(n)\right)\right. \\
& \left.-\operatorname{Std}\left(P_{N N_{\text {On-line }}}(n-50) \ldots P_{N N_{\text {On-line }}}(n)\right)\right)
\end{aligned}
$$

where $l_{r}$ is the learning rate used to tune the roll rate feedback gain.

The Simulink ${ }^{\circledR}$ simulation scheme is shown in Figure 3-32. The aircraft was simulated with the modified lateral linear model and the roll rate response was decreased by half to simulate a failure condition. 


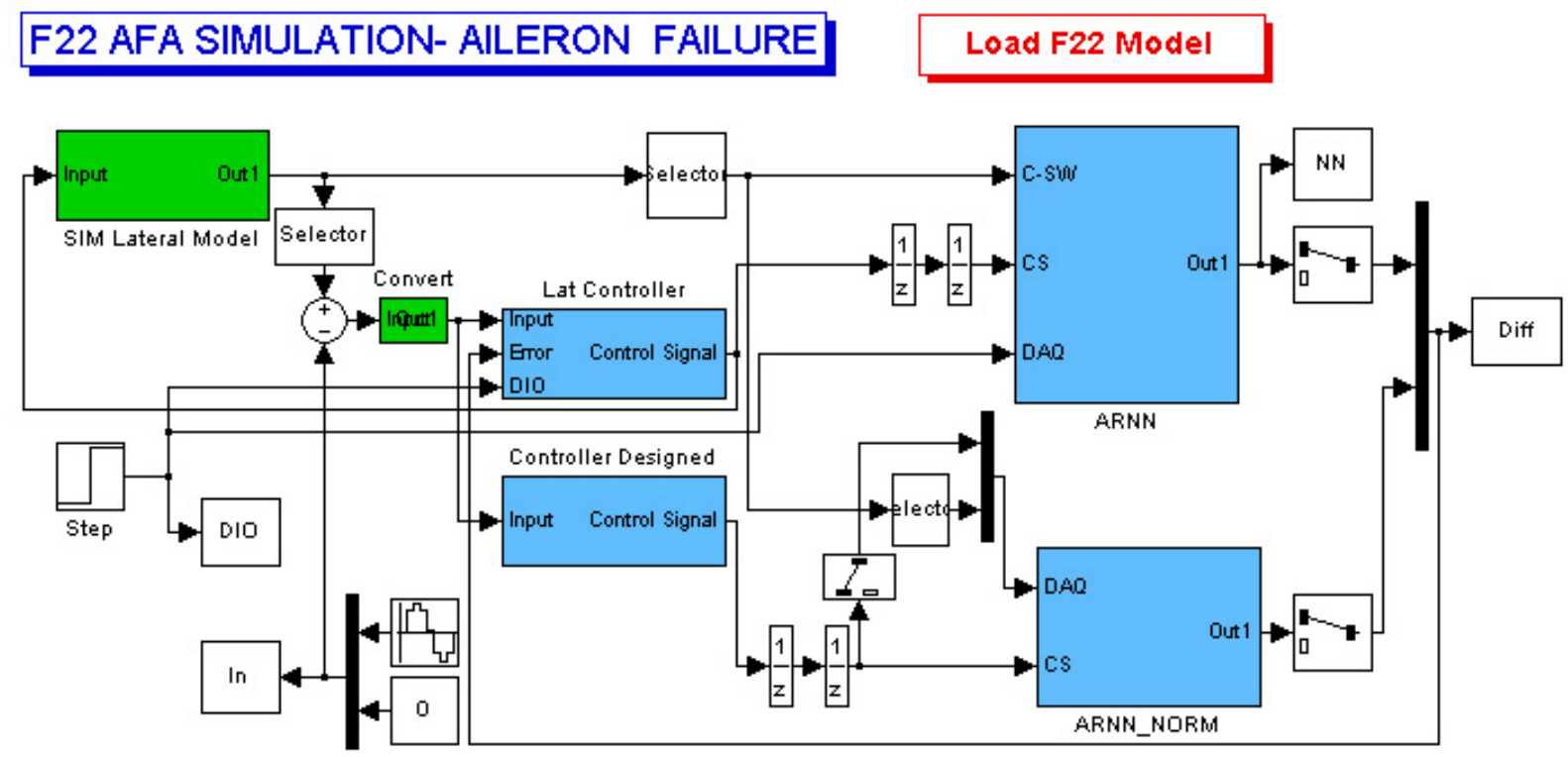

Figure 3-32 Aileron Failure AFA Simulation

The simulation results are shown in Figure 3-33. The simulation lasted for 500 seconds with the failure triggered at 100 second. The sampling rate for the simulation was set at $50 \mathrm{~Hz}$.
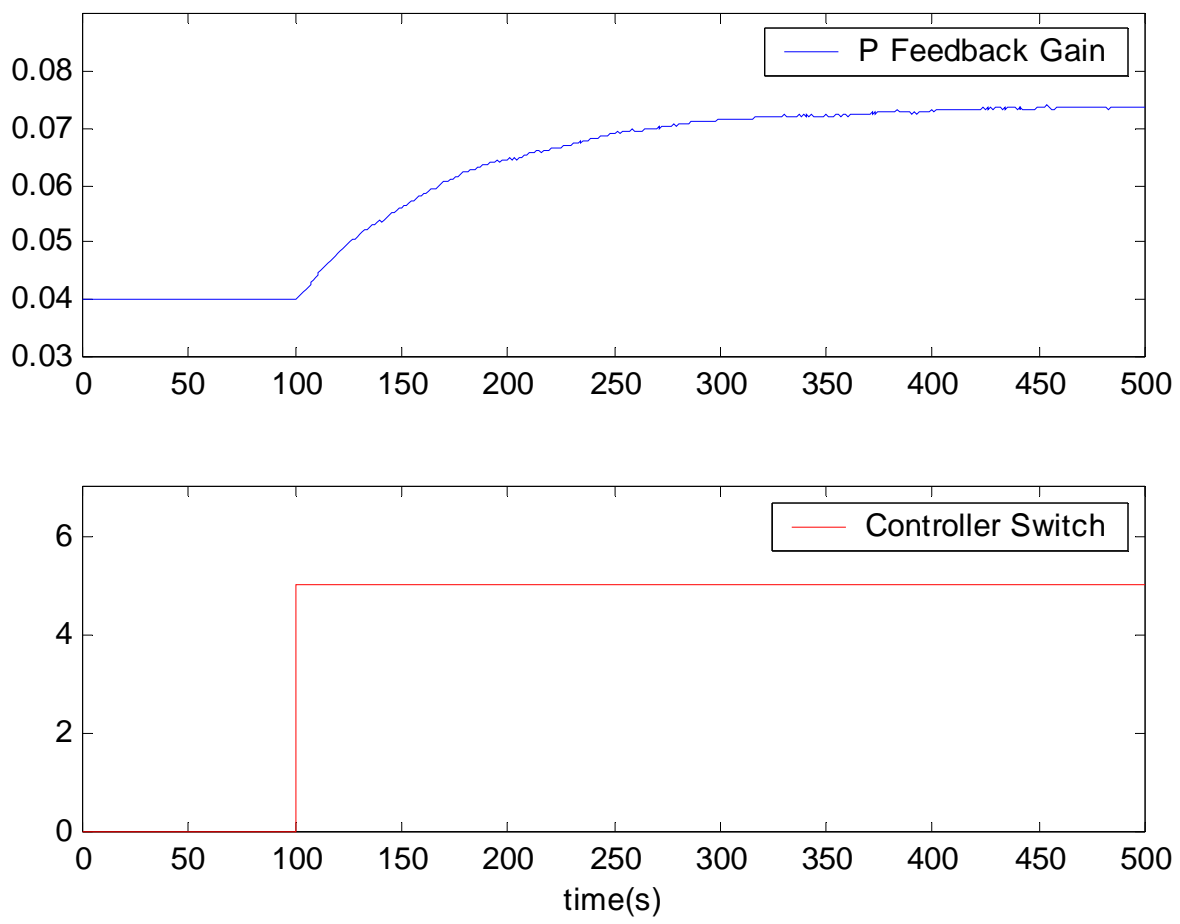

Figure 3-33 Aileron Failure AFA Controller Simulation - Gain Updating 
Once the failure was triggered, the on-line neural network began learning. The error between the on-line and off-line NNs was used to calculate the modeling error and tune the linear controller. The roll rate feedback gain (started at 0.04) was adjusted during the iteration process and eventually stabilized around 0.736. At the same time, the AFA controller gradually gained the capability to compensate for the loss of the right aileron. The performance comparison of linear controller without failure, linear controller with aileron failure, and the NN AFA controller with failure were simulated and presented in Figure 3-34. It can be seen that without the learning ability, the linear controller's performance degrades significantly. While the NN based AFA controller compensated for the failure and provided a similar performance as the linear controller without failure.

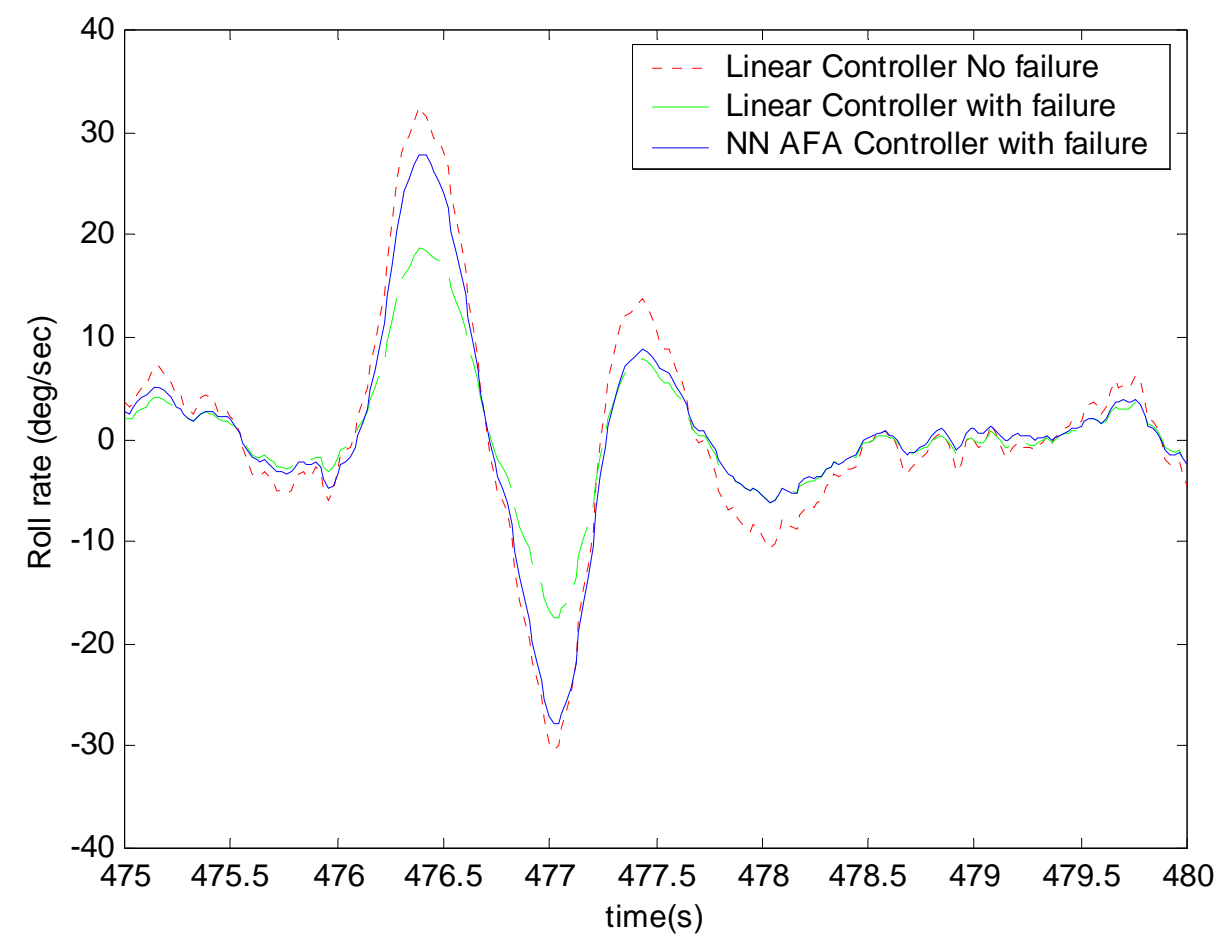

Figure 3-34 Performance Comparison of Three Conditions - P Channel 


\section{6 - Elevator Failure AFA Controller Design}

In the case of elevator failure, the right elevator was locked in a trim position while the left elevator remained functional. Flight tests were completed with this configuration under ground pilot control. Once the controller switch was activated, the right elevator would lock at the trim position and the pilot could then trigger elevator maneuvers with the fully functional left elevator. It can be observed from the collected flight data that during the elevator failure, two major adverse effects typically developed:

1. Elevator's control authority degrades; this is similar to the case with aileron failure since the effective elevator area had been decreased into half. Flight data shown in Figure 3-35 shows the difference of pitch rate response with both the failure and non-failure conditions. The solid line shows the pitch rate response after a \pm 7 degree elevator doublet with right elevator failure and the dot line shows the response of the same maneuver without failure. It is clearly shown that with the right elevator locked, the pitch rate response caused by the same pilot command was decreased to almost half.

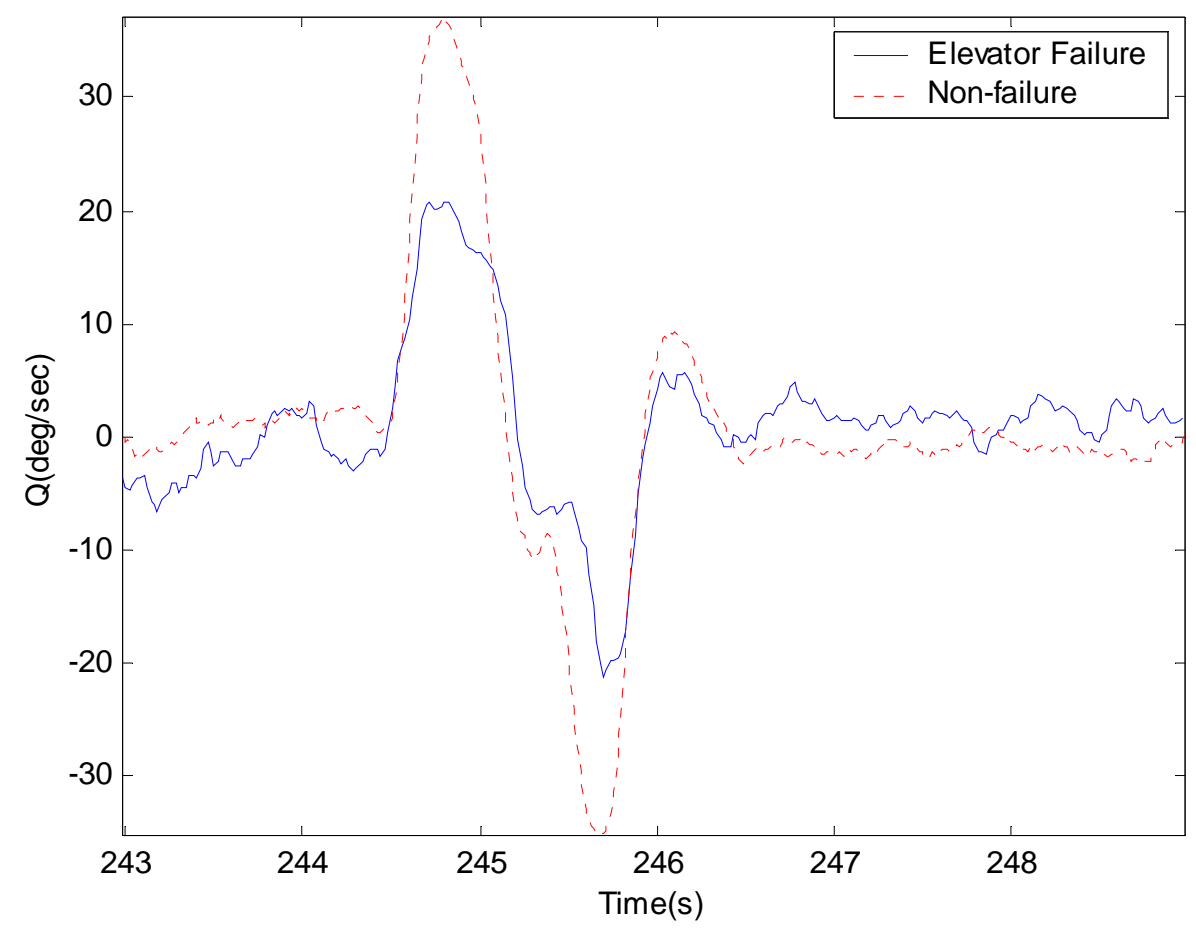

Figure 3-35 Elevator Failure Response - Q Channel 
2. Unsymmetrical elevator deflection caused a substantial rolling moment as expected. This is due to the moment arm from the $\mathrm{x}$-axis and the center of application of the force generated by the 'healthy' stabilator. This effect is shown in Figure 3-36. With the right elevator locked at trim position, the left elevator doublet caused a significant response in lateral channels. This effect is visually noticeable during the flight test. It can be seen that a \pm 7 degree elevator doublet could cause $60 \mathrm{deg} / \mathrm{sec}$ rolling moment.
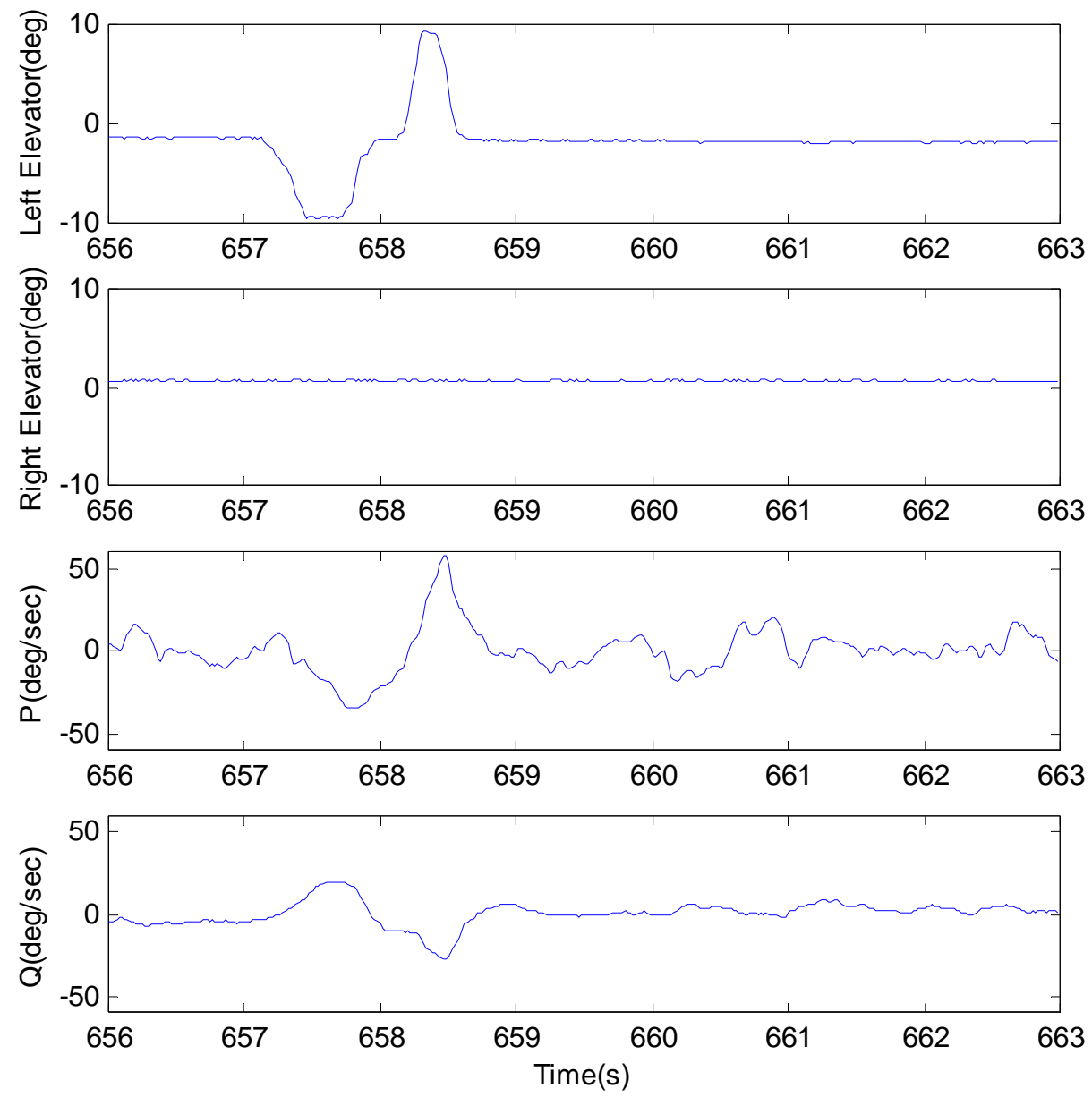

Figure 3-36 Elevator Failure Response - Coupling

This strong coupling effect can be explained by the relatively large size and position of elevators on the YF-22 model; the rolling moment caused by one side elevator was significant and must be compensated for so the controller can guarantee a satisfactory 
handling quality. It is clear that strong coupling between the longitudinal and lateraldirectional dynamics increased the complexity of the control problem.

To evaluate the performance of the linear controller under elevator failure, flight tests were performed with a similar method as with the aileron failure. A sine-wave pitch rate command was sent to the controller and aircraft responses are shown in Figures 3-37 though 3-39. Similar to the case of aileron failure, once the elevator failure had been triggered, the linear controller slightly increased the control command on the left elevator (Figure 3-37). This is because of a higher tracking error; however, a significant degradation on performance is still noticeable (Figure 3-38). The action of the linear controller also caused a rolling moment which is shown in Figure 3-39, where the solid line represents elevator failure and the dot line shows the response without failure (which actually shows a weak coupling with reversed sign caused by the imperfection of the aircraft). This is because of the strong coupling between the longitudinal and lateraldirectional dynamics after the elevator failure; as explained, the linear controller had no capability to compensate for this effect.

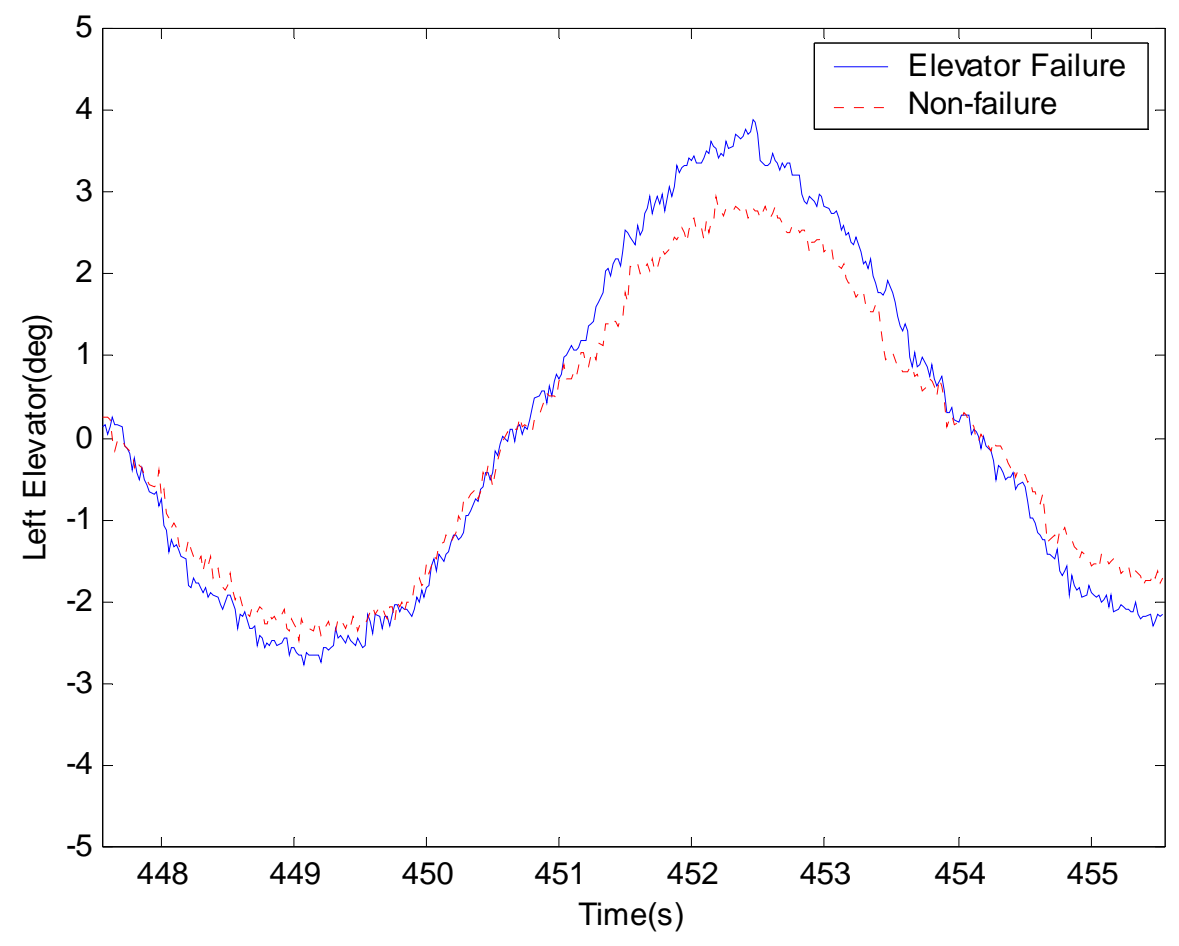

Figure 3-37 Linear Controller with/without Failure - Left Elevator 


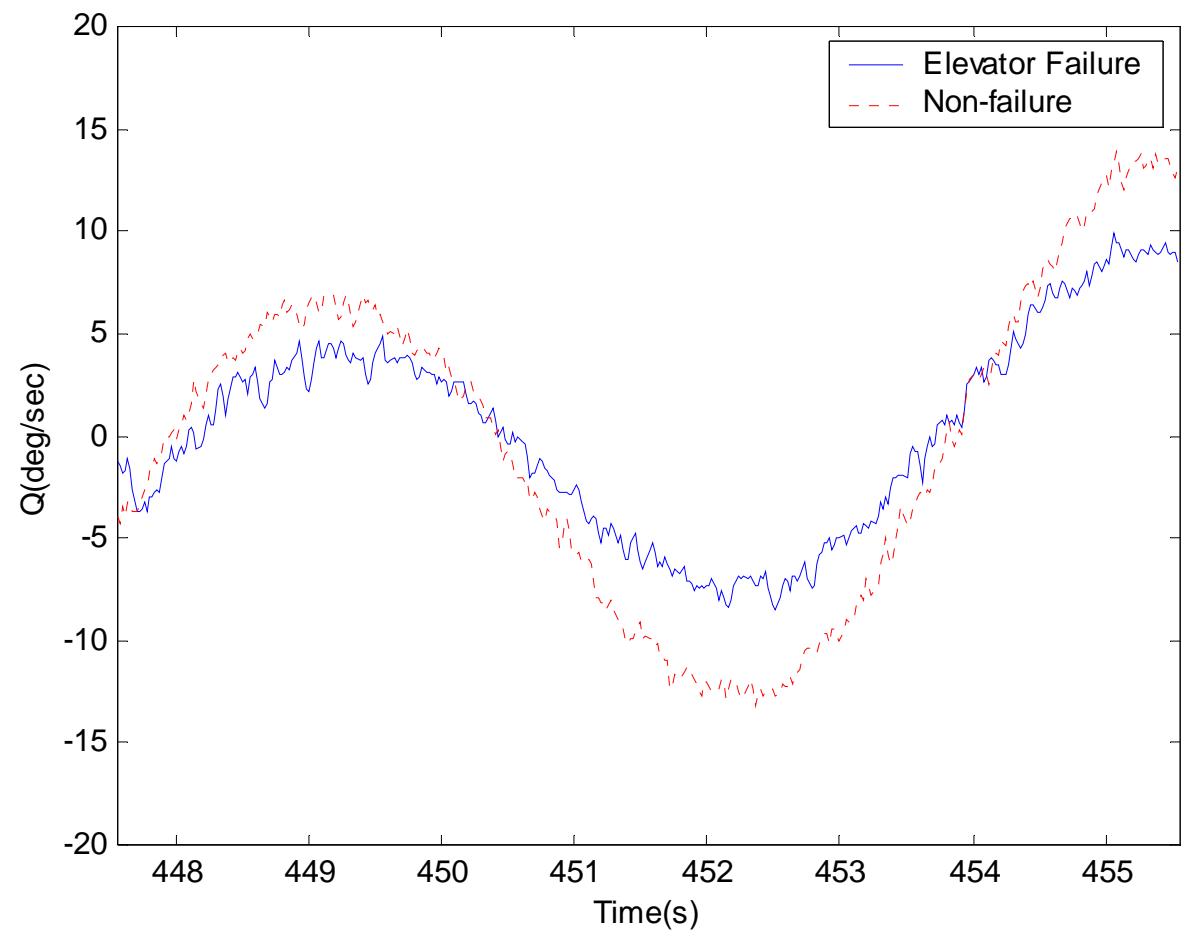

Figure 3-38 Linear Controller with/without Failure - Q Channel

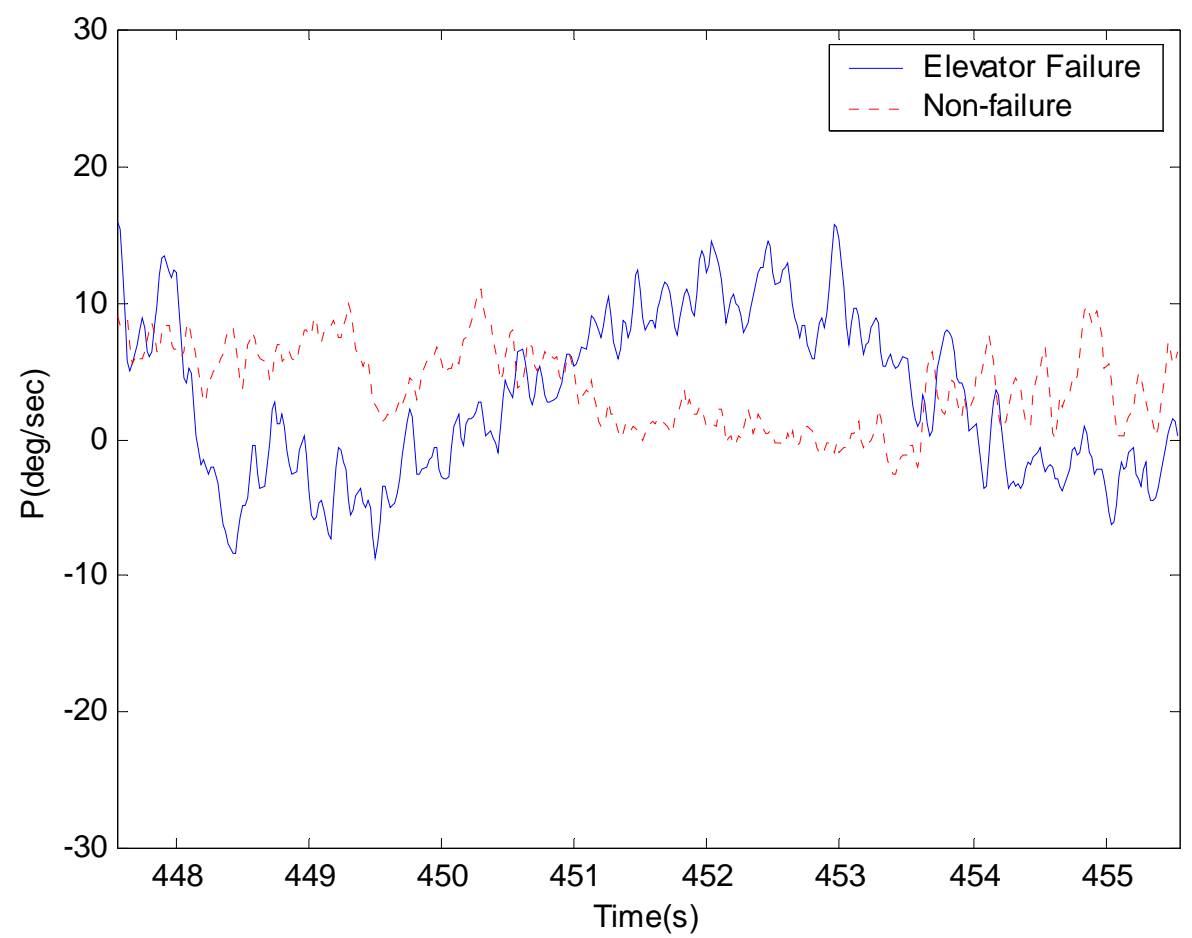

Figure 3-39 Linear Controller with/without Failure - P Channel 
To solve the linear controller's inability to handle the failure condition, an AFA controller had been designed to handle the elevator failure. The diagram of the controller design is shown in Figure 3-40.

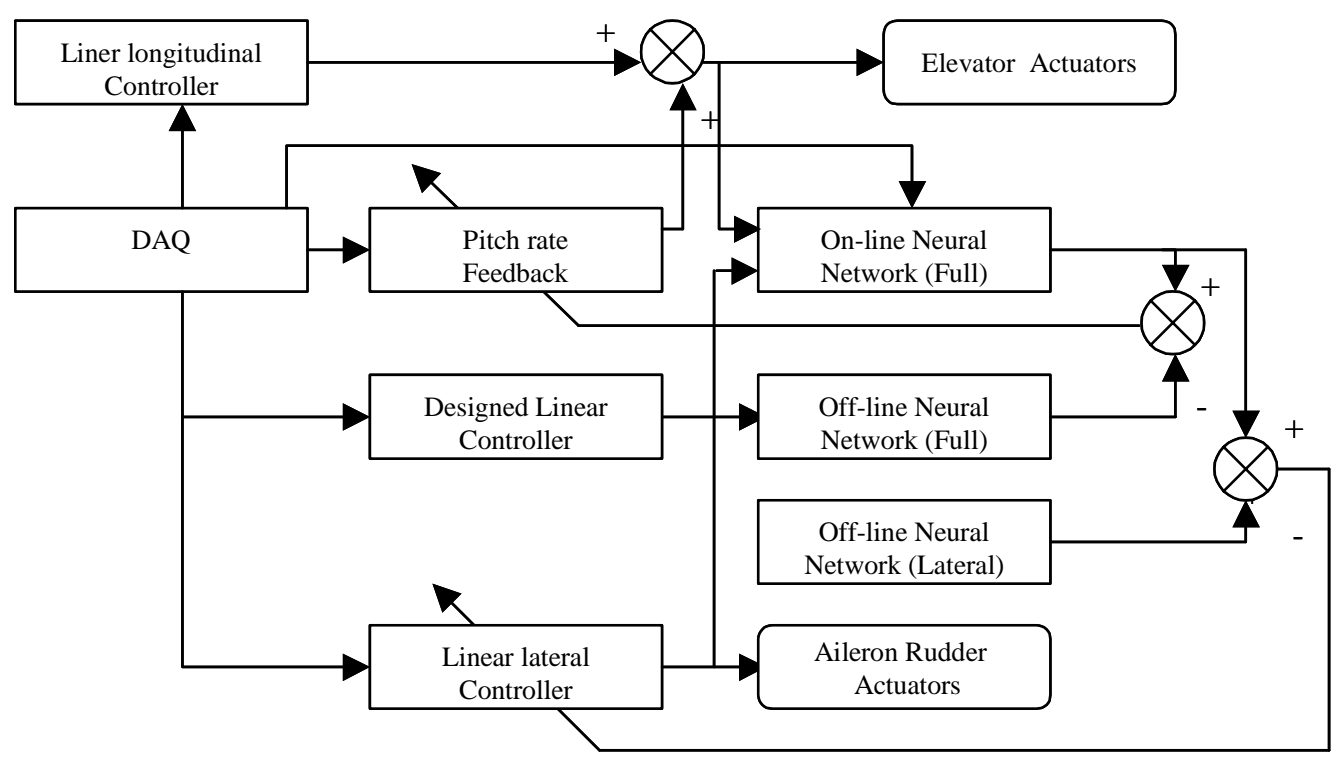

Figure 3-40 Elevator Failure AFA Controller Design

Two NNs were designed to approximate the aircraft full dynamics (longitudinal and lateral-directional): off-line learning $\mathrm{NN}$ for providing a reference response of the nominal flight condition, and on-line learning $\mathrm{NN}$ for approximating the aircraft dynamics after failure. The standard deviation of the pitch rate estimation of both NNs had been used to calculate the modeling change on the longitudinal channel and tune the linear pitch rate feedback gain. The gain tuning procedure can be modeled by:

$$
\begin{aligned}
& K_{q}(n+1)=K_{q}(n)+l_{r} \times\left(\operatorname{Std}\left(Q_{\text {FullNN }_{\text {Off-line }}}(n-50) \ldots Q_{\text {FullNN }_{\text {Off-line }}}(n)\right)\right. \\
& \left.-\operatorname{Std}\left(Q_{\text {FullNN }_{\text {On-line }}}(n-50) \ldots Q_{\text {FullNN }_{\text {On-line }}}(n)\right)\right)
\end{aligned}
$$

To compensate for the coupling between the elevator input and the lateral dynamics of the aircraft, an off-line learning NN to approximate the lateral-directional dynamics at nominal flight condition was used to provide a reference response. This NN is the same as the one used for the aileron failure AFA controller; so it is fully decoupled with the longitudinal dynamics of the aircraft. The standard deviation of the estimation of roll channel from both lateral off-line NN and full on-line learning NN was used to measure the coupling and tune the linear feedback gain from the elevator command to 
aileron command to compensate for the rolling-moment caused by the elevator failure. The decoupling gain tuning procedure can be modeled by:

$$
\begin{aligned}
& K_{d c}(n+1)=K_{d c}(n)+l_{r} \times\left(\operatorname{Std}\left(P_{\text {LateralNN }_{\text {Off-line }}}(n-50) \ldots P_{\text {LateralNN }_{\text {Off-line }}}(n)\right)\right. \\
& \left.-\operatorname{Std}\left(P_{\text {FullNN }_{\text {On-line }}}(n-50) \ldots P_{\text {FullNN }_{\text {On-line }}}(n)\right)\right)
\end{aligned}
$$

A NN to approximate both longitudinal and lateral-directional dynamics was designed for the elevator failure AFA controller. The aircraft states used by the NN training include:

- aileron deflection;

- rudder deflection;

- elevator deflection;

- angle of attack;

- angle of Sideslip;

- roll rate;

- $\quad$ pitch rate;

- yaw rate.

The selection of the neural network inputs at step $\mathrm{k}$ are shown in Table 3-3:

\begin{tabular}{|l|l|l|l|l|l|l|l|}
\hline$\delta_{\mathrm{a}}(\mathrm{k})$ & $\delta_{\mathrm{r}}(\mathrm{k})$ & $\delta_{\mathrm{e}}(\mathrm{k})$ & & & & & \\
\hline$\delta_{\mathrm{a}}(\mathrm{k}-1)$ & $\delta_{\mathrm{r}}(\mathrm{k}-1)$ & $\delta_{\mathrm{e}}(\mathrm{k}-1)$ & $\alpha(\mathrm{k}-1)$ & $\beta(\mathrm{k}-1)$ & $\mathrm{P}(\mathrm{k}-1)$ & $\mathrm{Q}(\mathrm{k}-1)$ & $\mathrm{R}(\mathrm{k}-1)$ \\
\hline$\delta_{\mathrm{a}}(\mathrm{k}-2)$ & $\delta_{\mathrm{r}}(\mathrm{k}-2)$ & $\delta_{\mathrm{e}}(\mathrm{k}-2)$ & $\alpha(\mathrm{k}-2)$ & $\beta(\mathrm{k}-2)$ & $\mathrm{P}(\mathrm{k}-2)$ & $\mathrm{Q}(\mathrm{k}-2)$ & $\mathrm{R}(\mathrm{k}-2)$ \\
\hline
\end{tabular}

Table 3-3 Inputs of Full Neural Networks

Where $\alpha(\mathrm{k}), \beta(\mathrm{k}), \mathrm{P}(\mathrm{k}), \mathrm{Q}(\mathrm{k})$ and $\mathrm{R}(\mathrm{k})$ were used to evaluate and train the $\mathrm{NN}$.

The selected NN was a MLP with BP training algorithm. To guarantee on-line training speeds and minimize the overfitting problem, the size of the hidden layer was selected to be small (7 hidden neurons). Thus, the structure of the NN include:

- 19 input neurons;

- 7 hidden neurons;

- 5 output neurons.

The NN was trained off-line with the flight data collected from nominal flight conditions; the weight and threshold information were stored in a data file. The on-line and off-line $\mathrm{NN}$ used in the controller to approximate the full dynamics of the aircraft had the same 
structure and used the same weight/threshold value at the starting stage of the simulation. Once the failure was triggered, the on-line learning $\mathrm{NN}$ would start training to approximate the post failure dynamics.

Simulation tests were performed with the designed elevator failure AFA controller. The Simulink® simulation scheme is shown in Figure 3-41. The aircraft was simulated with the modified longitudinal linear model, with the pitch rate response being decreased to half to simulate a failure condition. A coupling constant of 0.4 between the elevator command and aileron inputs was found to match the flight data with right elevator failure, and was used to simulate the aircraft's longitudinal/lateral coupling after failure.

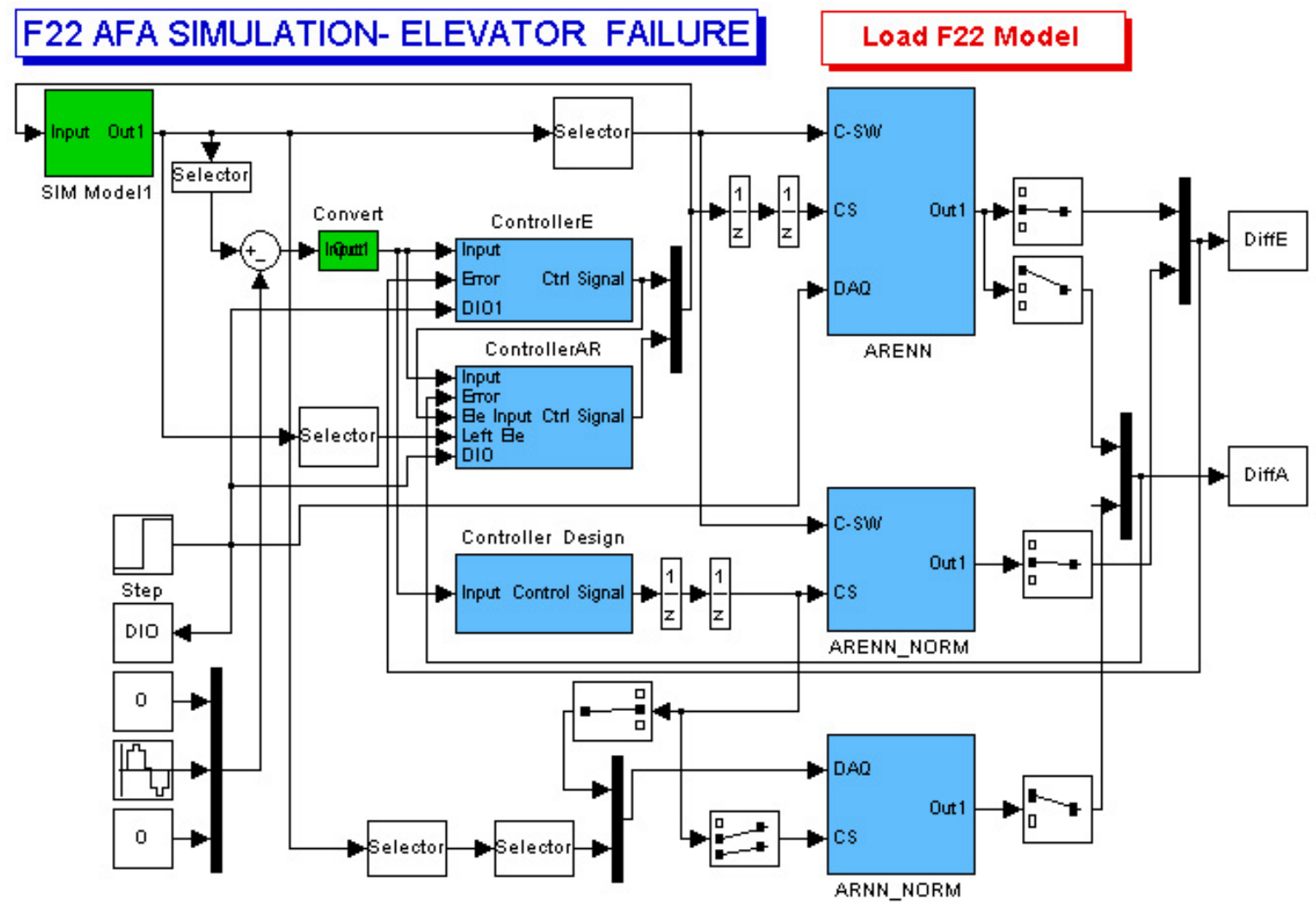

Figure 3-41 Elevator Failure AFA Simulation

The simulation results are shown in Figure 3-42. The simulation lasted for 500 seconds and the failure was triggered at 100 second. The sampling rate for the simulation was set at $50 \mathrm{~Hz}$. 

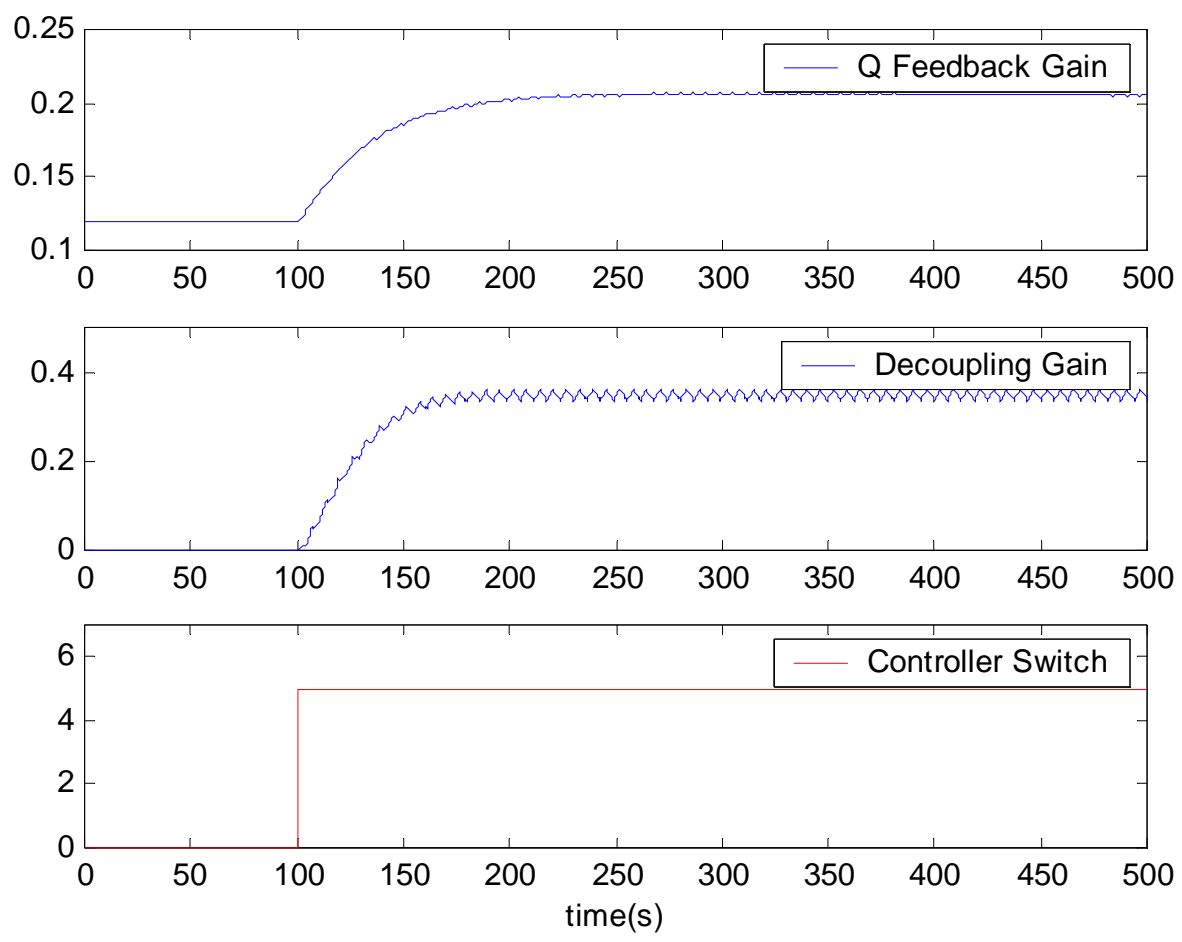

Figure 3-42 Elevator Failure AFA Simulation - Gain Updating

Once the failure was triggered, the on-line neural network began the learning phase. The error between the on-line and off-line NNs was used to calculate the modeling change and tuning of the feedback gains. The pitch rate gain - starting at 0.12 was adjusted during the iteration process and eventually stabilized around a value of 0.205. At the same time, the AFA controller gradually gained the capability to compensate for the loss of the right elevator. The left elevator/lateral coupling gain, starting at 0 had been increased during the learning process where it eventually stabilized around a value of 0.35 . The performance comparison of linear controller without failure, linear controller with elevator failure, and the NN AFA controller with failure was presented in Figures 3-43 and 3-44. Figure 3-43 shows the simulated pitch rate response. While the NN was learning, the designed AFA controller shows significant improvement of roll rate response over the linear controller. The simulated roll rate response in Figure 3-44 shows the NN AFA controller effectively compensates for the coupling from the left elevator while the unwanted lateral response was decreased to a very small value. The handling quality of the aircraft after failure was improved with the designed AFA controller. 


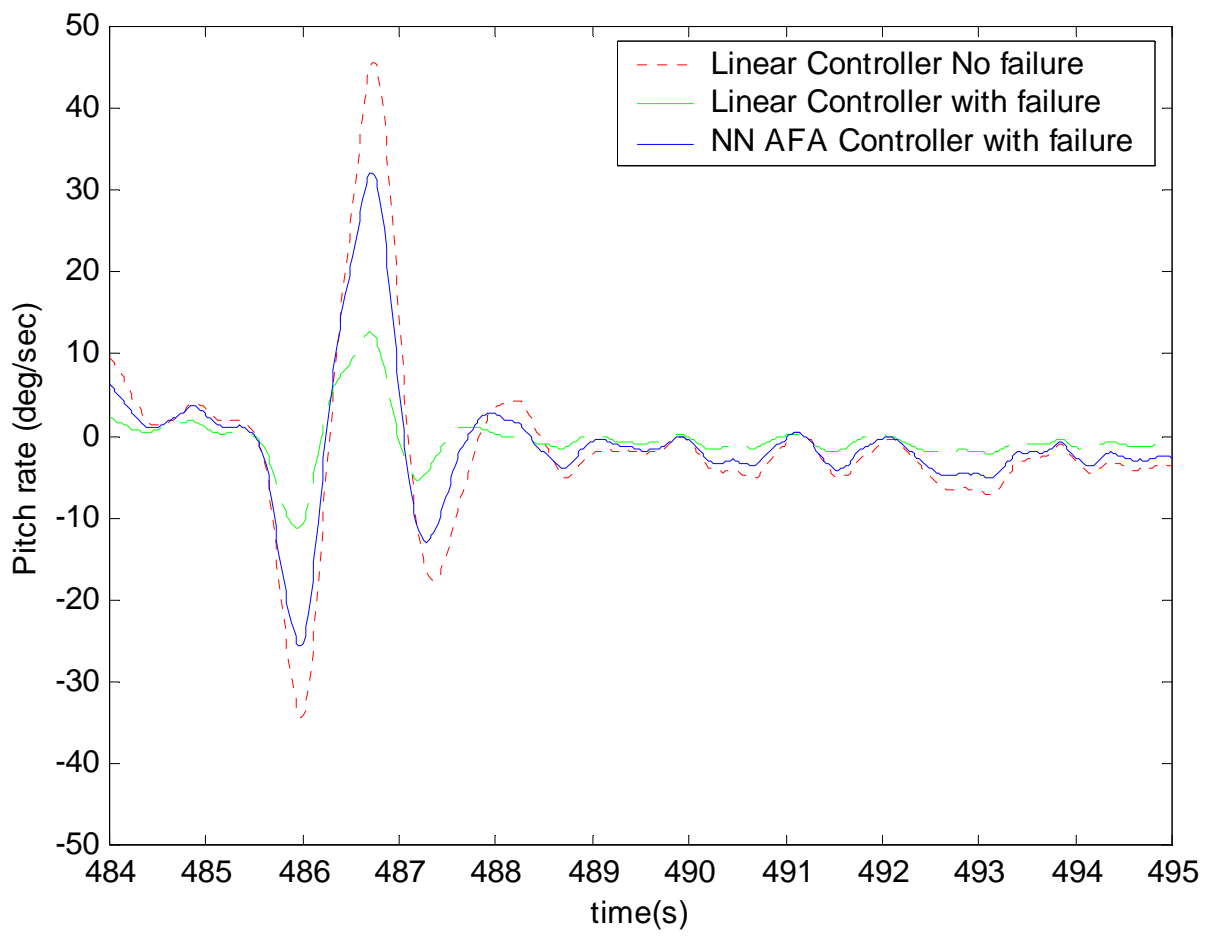

Figure 3-43 Elevator Failure AFA Simulation - Q Channel

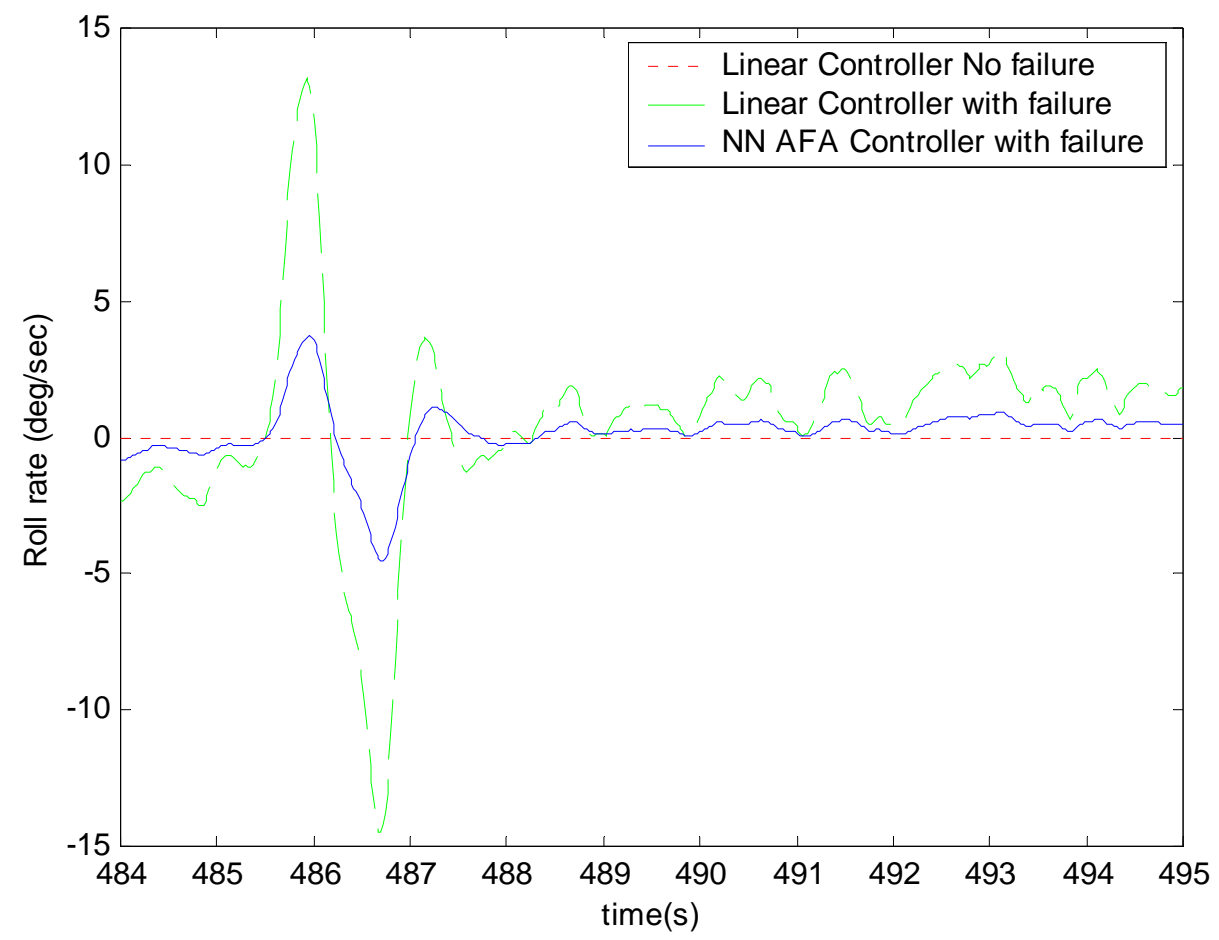

Figure 3-44 Elevator Failure AFA Simulation - P Channel 
From the simulation results above, the AFA controller designed in this chapter shows promising performance in accommodating for the actuator failure. How to implement these controller designs into the flight testing experiments and validate the simulated controller performance had been a major issue in this research effort. Details about the development of the test-bed aircraft, on-board hardware/software, and flight testing activities will be discussed in the following chapters. 


\section{Chapter 4 Test-bed Aircrafts \& On-board Payload}

To fully evaluate the actuator failure conditions and validate the performance of the designed AFA controller, aircraft test-beds were initially developed. On-board electronic payload systems were designed around the aircraft systems to perform data acquisition, failure condition triggering and control of the aircraft. This section provides details about the aircraft test-bed and the on-board hardware used for the flight testing experiments.

\subsection{Test-bed Aircrafts}

The WVU YF-22 jet powered research UAVs were developed for flight testing of the controller designed. This project was initially started with "Version 1" of the WVU YF-22 shown in Figure 4-1.

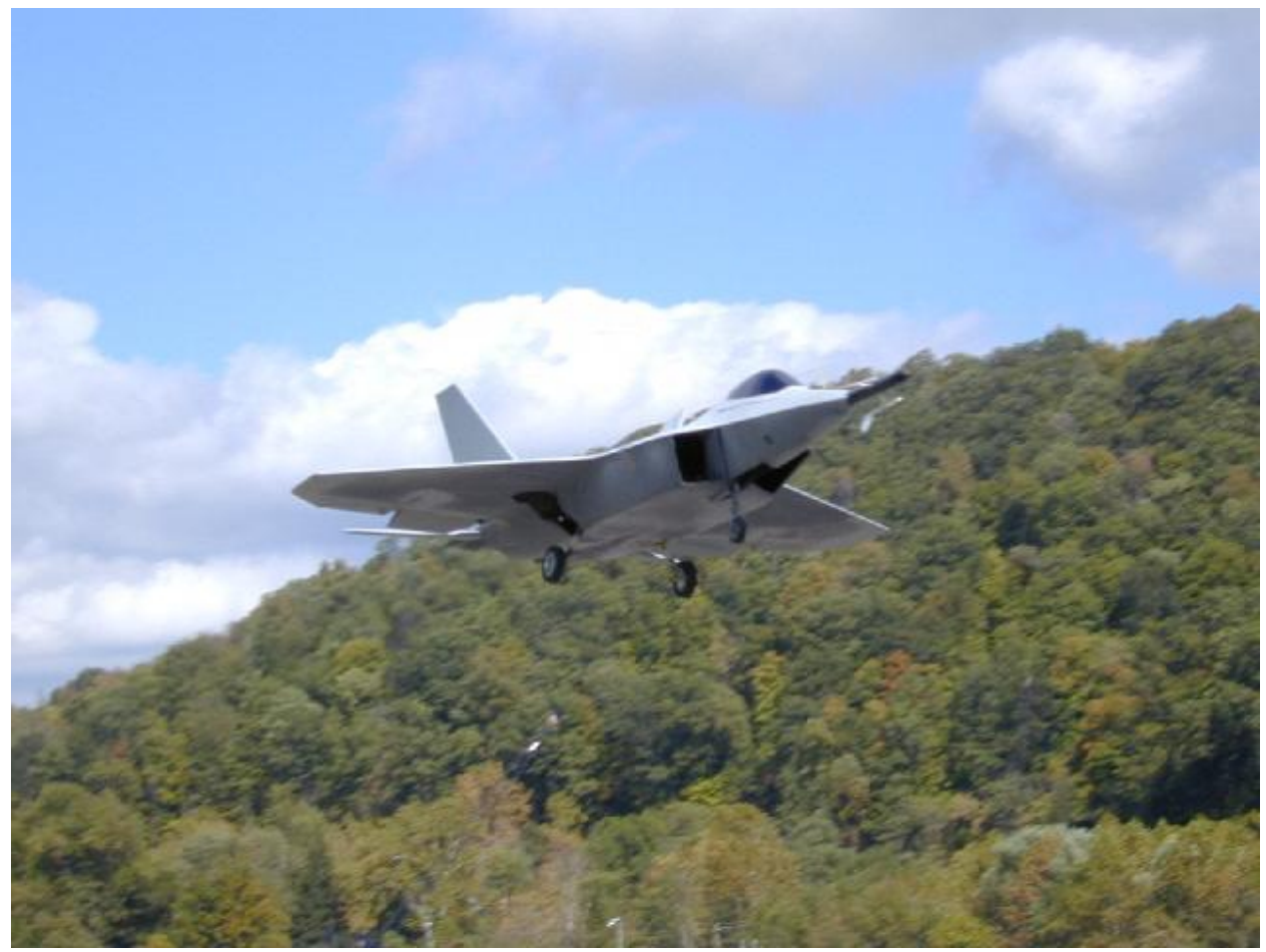

Figure 4-1 WVU YF-22 Research Model Aircraft (Version 1)

On-board instrumentation and control hardware adequate for actuator failure triggering and accommodation were developed for these UAVs. About 36 flight tests were 
performed on this particular aircraft including aircraft evaluation, data acquisition, failure simulation and linear controller validation.

With the starting of the formation flight project a new fleet of improved YF-22 UAVs was developed. A picture of ship green at take-off is shown in Figure 4-2.

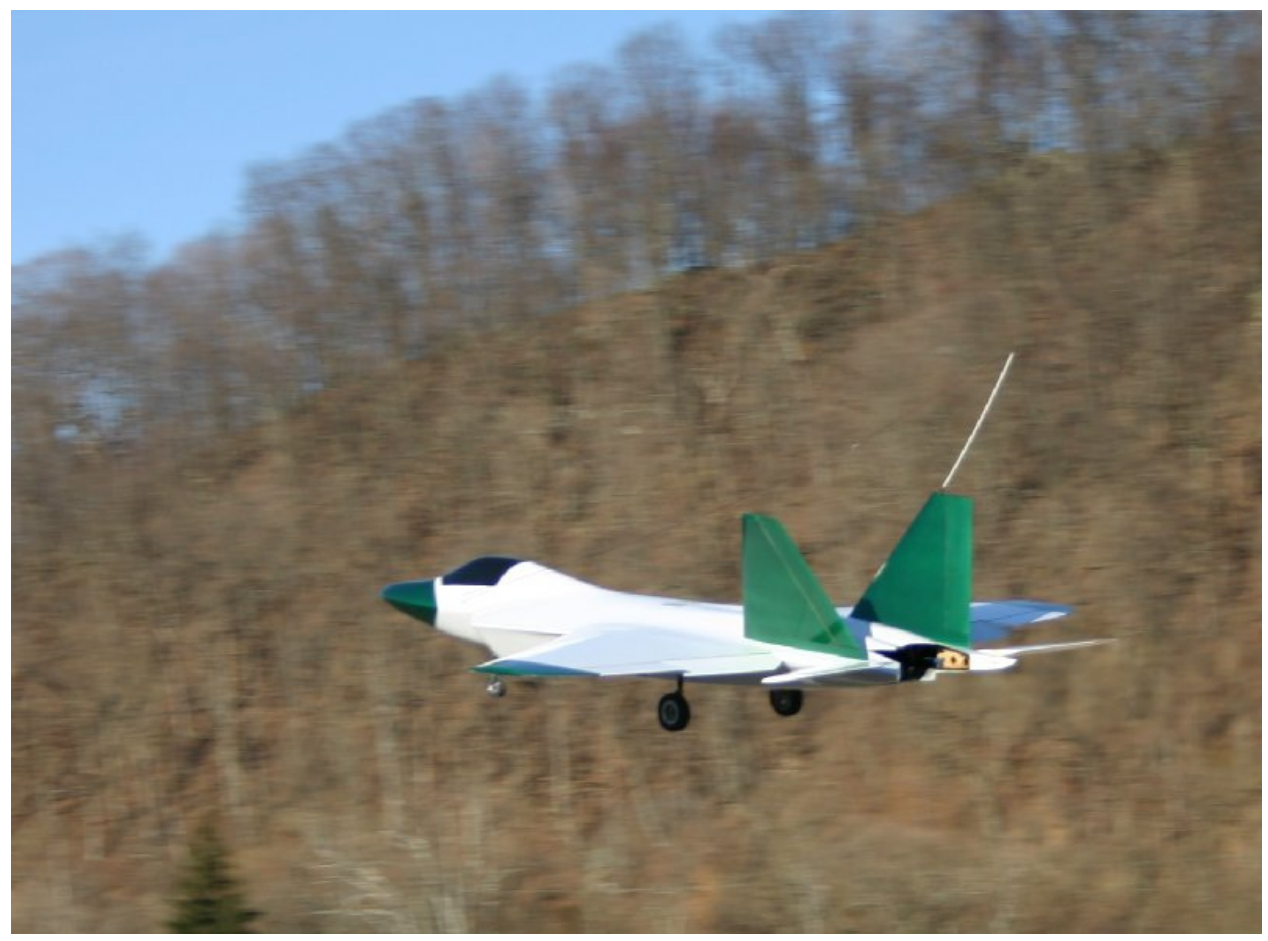

Figure 4-2 WVU YF-22 Research Model Aircraft (Version 2)

The new version of YF-22 UAVs have a larger avionics bay capable of carrying an even heavier payload with increased flight mission duration. A vertical gyro and GPS unit was added to the new YF-22 on-board payload for formation flight testing to get a better measurement of aircraft's parameters. This additional information benefited engineers for both aircraft capabilities and data analysis. The AFA software was then transferred to one aircraft in the new fleet. Flight testing activities included data acquisition, failure analysis and actuator failure accommodation had been completed.

As a flying test-bed, the aircraft's performance had a direct relationship to the design of the on-board payload and controller. The aircraft frame was made of composites fiberglass, carbon fiber, Kevlar, foam, and wood. Unlike the equivalent models used in recreational purposes, a perfectly scaled research model was not required and did not exhibit acceptable handling qualities due to the negative effect of the payload on the dynamic characteristics. Therefore, specific changes were made to the geometric 
and aerodynamics characteristics of the aircraft model to achieve desirable handling qualities in the model. In particular, the design was aimed at achieving acceptable values for two specific parameters, the thrust/weight (T/W) ratio and the weight/wing surface $(\mathrm{W} / \mathrm{S})$ ratio.

\subsection{1 - Aircraft Specifications}

The WVU YF-22 research aircraft model (Version 2) has an approximate 6'6" wing span and is powered by a miniature jet engine with $28 \mathrm{lbs}$ thrust. The payload capability is about $12 \mathrm{lbs}$, which can easily carry the on-board computer system and all necessary instrumentation (Figure 4-3).

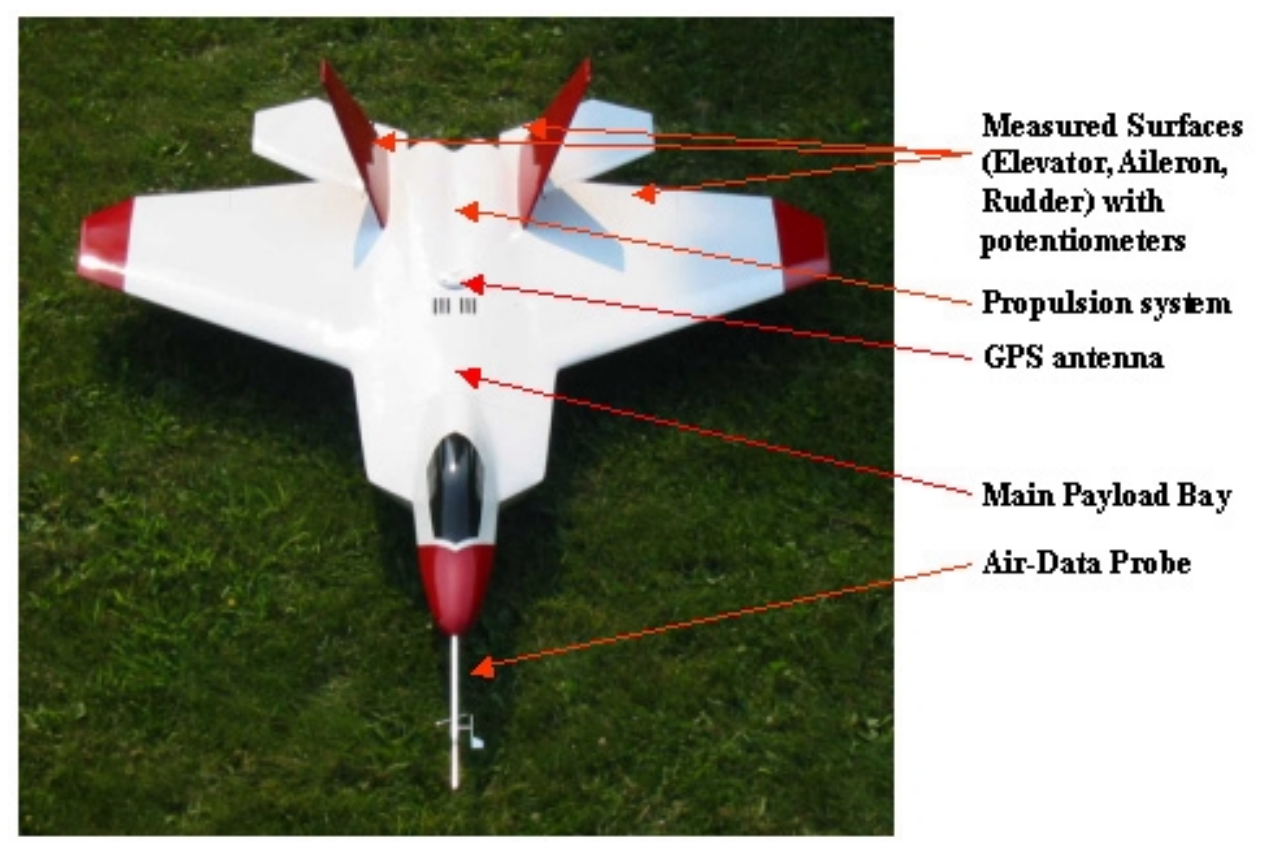

Figure 4-3 Aircraft Overview

The aircraft is controlled by a 10 -channel $\mathrm{R} / \mathrm{C}$ radio by the ground pilot with the on-board controller disengaged. Manual takeoff and landing are performed by the pilot. The pilot has the full control of elevators, ailerons, rudders, flaps, engine throttle, brakes, retractable landing gear, and a controller switch to activate the on-board controller. The fuel capability is about $7 \mathrm{lbs}$ with a typical mission duration of about 12 minutes. This ensures adequate flight time for the actuator failure test without putting a high level of stress on the UAV pilot. Specifications for the WVU YF-22 research UAVs are shown in Table 4-1: 


\begin{tabular}{|l|l|}
\hline Aircraft Parameters & Values \\
\hline Wingspan & $78^{\prime \prime}$ \\
\hline Length & $10 \mathrm{ft}^{\prime}$ with probe \\
\hline Height & $24{ }^{\prime}$ \\
\hline Wing Area & $14.7 \mathrm{ft}^{2}$ \\
\hline Weight & $50 \mathrm{lbs}$ \\
\hline Payload Capability & $10-12 \mathrm{lbs}$ \\
\hline Fuel Capability & $71 \mathrm{bs}$ \\
\hline Duration & $12 \mathrm{minutes}$ \\
\hline Cruse Airspeed & $90 \mathrm{mph}$ \\
\hline Takeoff Speed & $60 \mathrm{mph}$ \\
\hline Radio & $\mathrm{JR} 10 \mathrm{X} 10$ channel SPCM \\
\hline Engine & RAM1000 \\
\hline Thrust & $281 \mathrm{bs}$ \\
\hline T/W ratio & 0.56 \\
\hline W/S ratio & $54 \mathrm{oz} \mathrm{ft}^{2}$ \\
\hline
\end{tabular}

Table 4-1 YF-22 Research UAV Specifications

\subsection{On-board Payload}

The on-board electronic payload system was designed to monitor and control the UAV. The on-board sensors provide measurements of all the major parameters of the aircraft. The on-board electronic package performs data acquisition, execution of the control laws and distributes control signals to the individual aircraft control actuators. Six-controller channels were provided by the on-board controller including: left elevator, right elevator, left aileron, right aileron, rudders and the engine throttle. To perform the actuator failure accommodation the following specific requirements were used to define the system architecture:

1. Different commands (control commands, failure, start/stop of learning) can be sent from the ground pilot; 
2. The On-Board Computer (OBC) can have full control of the major aircraft control surfaces;

3. At any time the pilot can regain direct control of the aircraft;

4. Actuator failure can be simulated during the flight;

5. Actuator failure can be accommodated with the use of on-board controller during the flight;

6. The package should be small, robust, resistant to vibration, operate in cold/hot/humid environments and easy to be installed with a combined weight of less than 10 lbs.

7. The package should have a minimum Electromagnetic Interference (EMI)

With these requirements, the selected hardware system was designed to be able to operate in the following three distinct modes of operation:

1. "Manual" Mode;

2. "Manual - Partial Automatic" Mode;

3. "Automatic" Mode.

In the "Manual" mode the pilot has complete control of the aircraft. The pilot can switch to this mode at any time when operating in the other two modes. This mode was designed to provide a safe operating condition before engaging the controller, and can be used for emergency recovery in case of controller instability. During the take off and landing phase of the mission, the aircraft was required to be in the manual operating mode. Within "Manual - Partial Automatic" Mode, the pilot has control over a subset of the aircraft main control surfaces while the on-board controller controls the remaining surfaces. The preprogrammed on-board software decides which control channels to be allocated to the on-board computer while the pilot retains control of the remaining channels. This mode was for intermediate testing of the actuator failure because it would minimize risk during flight tests and could be used to trigger certain maneuvers with pilot inputs. Within "Automatic" Mode, the control system has complete control of all major control surfaces. This mode was only used for the evaluation of linear controller design and the final phase of the actuator failure accommodation tests. 


\subsection{1 - On-board Payload Subsystems}

According to the requirements and the selected architecture, the on-board payload system was designed to receive pilot commands, collect flight data with the on-board instrumentation, generate on-board control commands and distribute control signals to control surfaces. In this way, the on-board payload (Figure 4-4) can be divided into three subsystems:

1. R/C System;

2. Data Acquisition System;

3. Control Signal Distribution System.

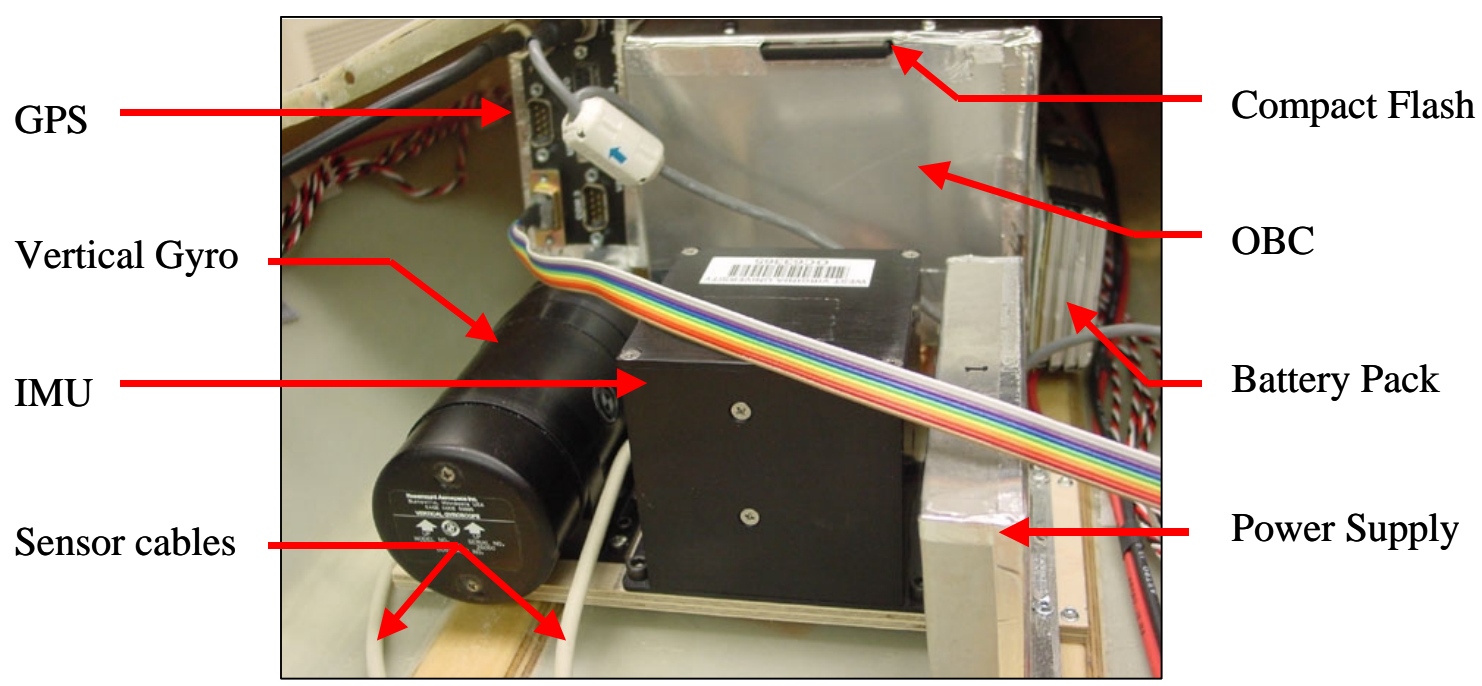

Figure 4-4 On-board Payload

\subsection{2 - R/C System}

The WVU YF-22 research aircraft is remotely controlled with JR 10X radio shown in Figure 4-5. 


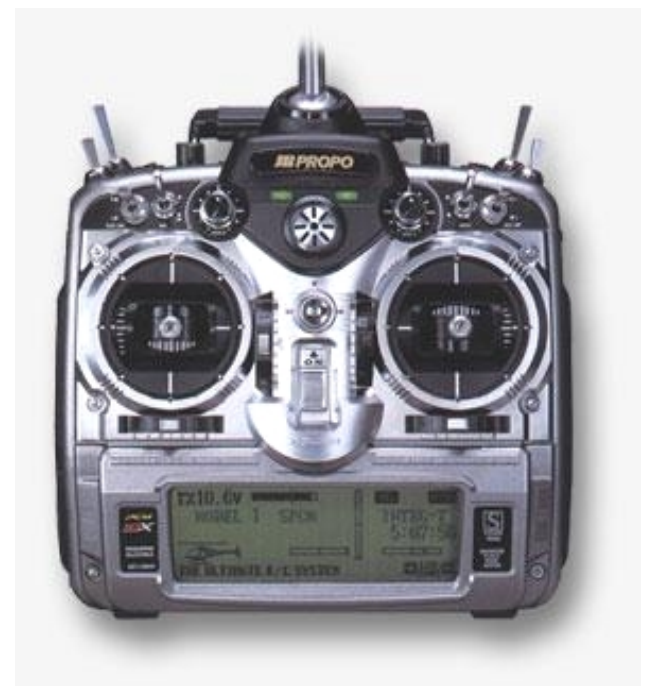

Figure 4-5 R/C Radio

The R/C receiver - shown in Figure 4-6 - was installed at the certain distance from the computer box to avoid potential EMI issues.

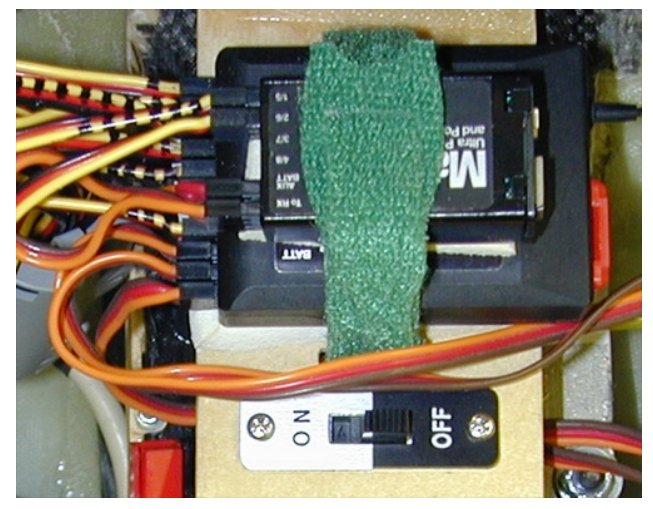

Figure 4-6 R/C Receiver

Nine cables were used to transmit the receiver control signals to the on-board computer. The Control Signal Distribution System (CSDS) inside the computer box distributes the control signals according to the on-board software and sends them to the individual servos of each control surface. The receiver set-up features 10 channels, including eight for controlling the aircraft, one for turning on/off the on-board controller, and one for acquiring the throttle command.

\subsection{3 - Data Acquisition System}

The data acquisition system monitors the condition of the aircraft and collects data for both controller and post-flight analysis purposes. The data acquisition system 
includes the on-board computer and instrumentation. The on-board instrumentation for the UAV model includes:

- Nose probe;

- Pressure sensors;

- Inertial Measurement Unit (IMU);

- Vertical gyro;

- Potentiometers on 6 major control surfaces $\left(\delta_{E_{L / R}}, \delta_{A_{L / R}}, \delta_{R_{L / R}}\right)$;

- Global Positioning System (GPS);

- Temperature sensor;

These above sensors provide measurements of the aircraft's angle of attack, angle of sideslip, static \& dynamic pressure, temperature, Euler angles, 3-axis angular rates, 3-axis accelerations, position, velocity, and the deflection of all control surfaces. The general architecture of the data-acquisition system is shown in Figure 4-7.

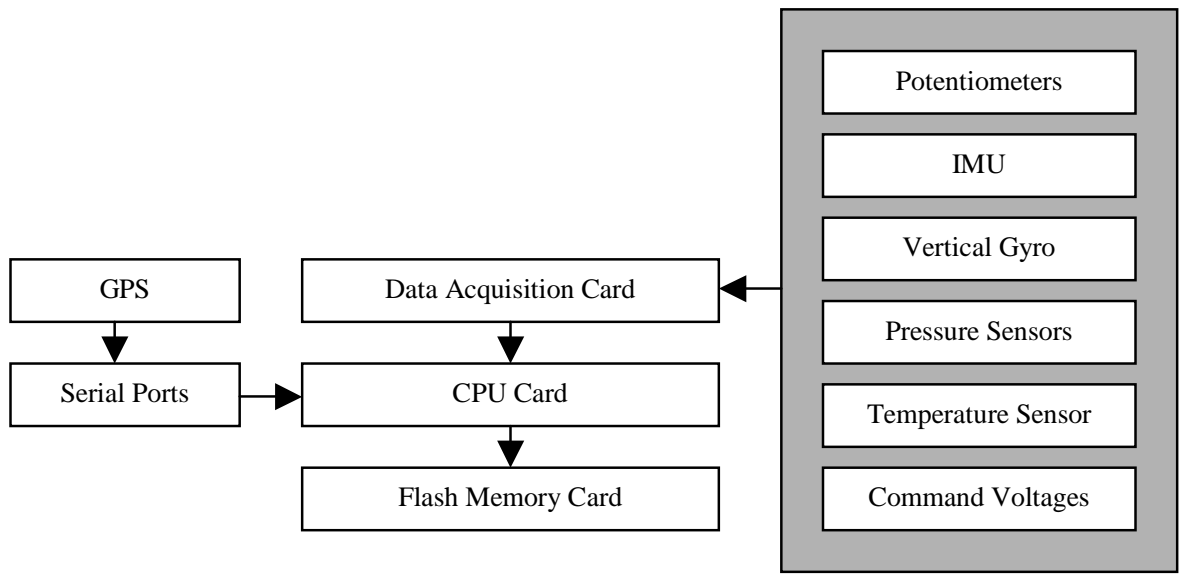

Figure 4-7 Data Acquisition System

The on-board computer is a PC104 format computer stack. This system includes a CPU card, data acquisition card, power supply card, custom sensor connection card, and a top panel for connection purposes to video/keyboard, etc. The layout of the OBC is shown in Figure 4-8. 


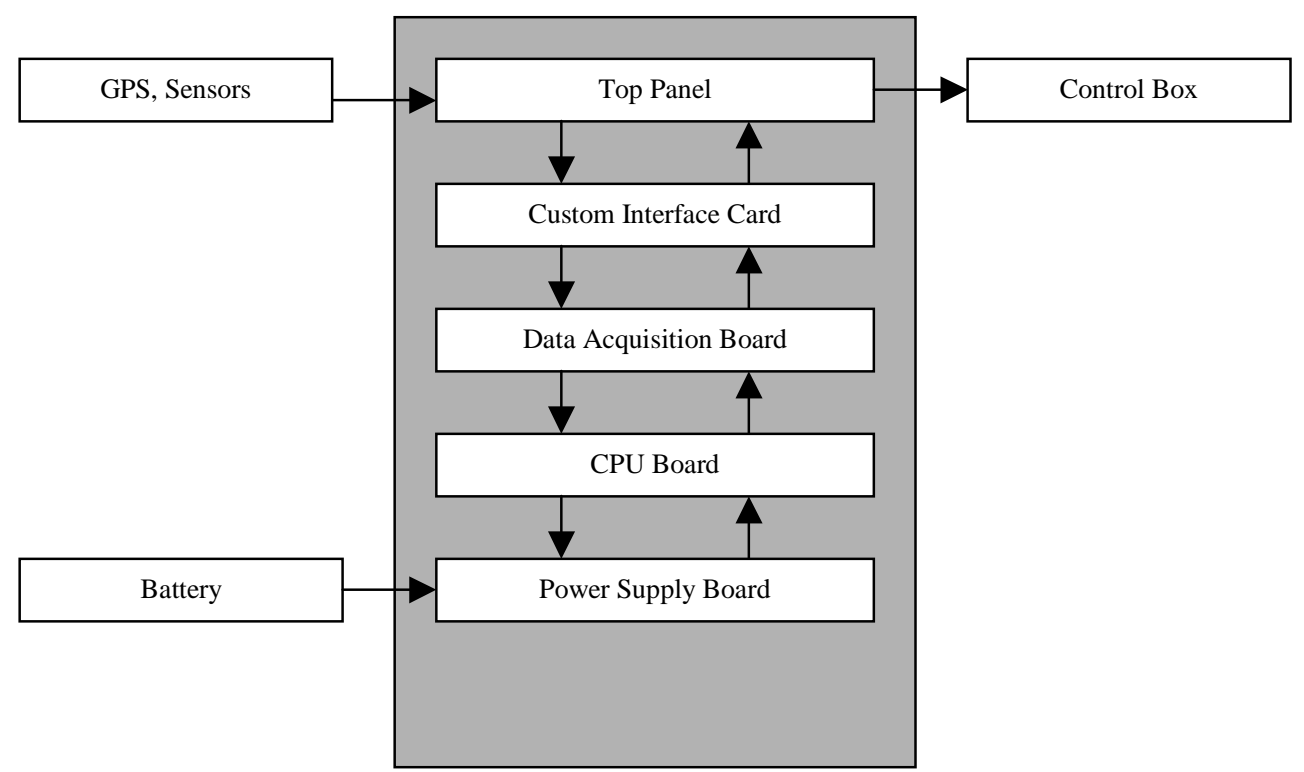

Figure 4-8 Layout of the $O B C$

The YF-22 data acquisition card has the capacity of 32 analog/digital channels of which 22 channels were used and with the remaining channels reserved for future expansions.

\subsection{4 - Control Signal Distribution System}

The main component of the control signal distribution system is the controller board, which acts as a hub for the whole flight control hardware. The functions of the CSDS include:

1. Receive control signals from the pilot;

2. Receive control signals from the $\mathrm{OBC}$;

3. Transfer the command from the OBC into PWM signals;

4. Select the current operation mode of the aircraft (Manual or Automatic);

5. Select channels to be controlled by the OBC;

6. Distribute control signals to individual servos.

In addition to all these functions, the controller board must be extremely reliable to guarantee aircraft safety. The pilot can use channel 7 of the transmitter to enter the "Automatic" Mode; however, he can reverse to manual mode at any time. The block diagram for the CSDS is shown in Figure 4-9. 


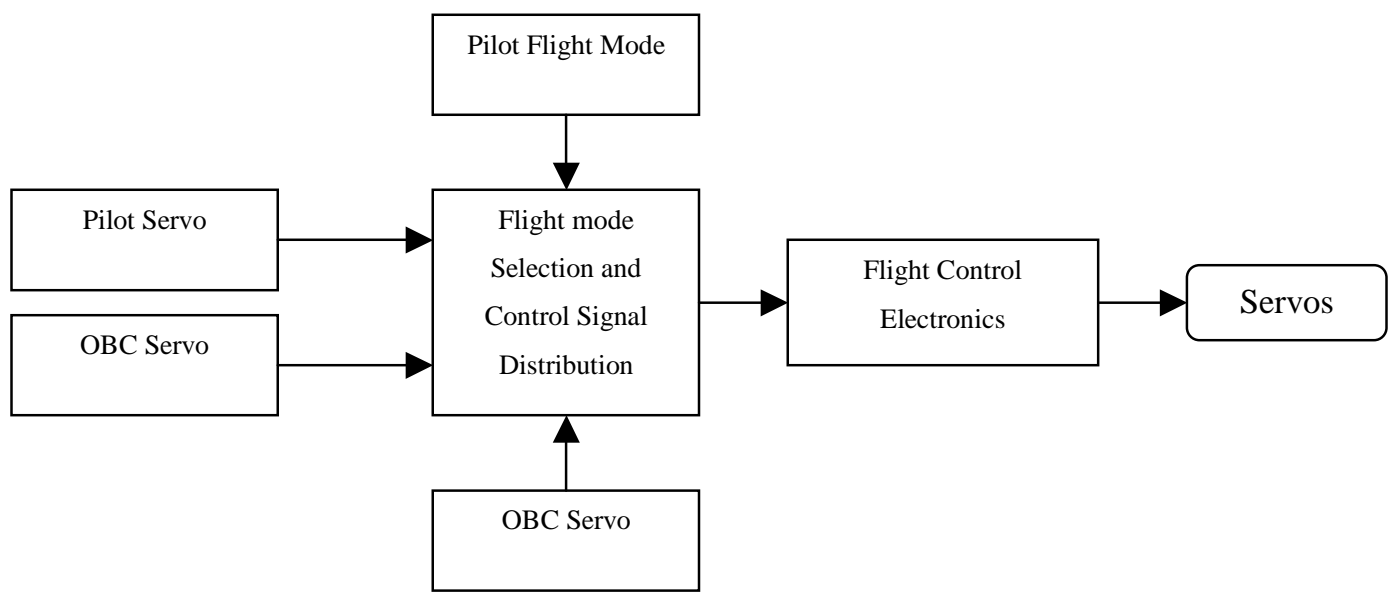

Figure 4-9 Control Signal Distribution System

A more detailed description of the hardware components is provided in Section 4.3.

\subsection{Major Components}

According to the architecture outlined in the previous section, the aircraft payload components were designed to have:

1. On-board computer modules to assemble the PC-104 on-board computer;

2. On-board instrumentation for acquiring the flight status of the aircraft;

3. Custom designed components for providing power, connecting sensors and servos with the on-board computer and distributing aircraft control signal.

This section will give a detailed description of major components used in the YF-22 research UAV on-board payload.

\subsection{1 - On-Board Computer Modules}

The OBC shown in Figure 4-10 is a PC-104 stack, which contains a CPU module, data acquisition module, power supply module and supporting components. The PC-104 format devices were selected because of their miniature size, lightweight, and low power consumption. 


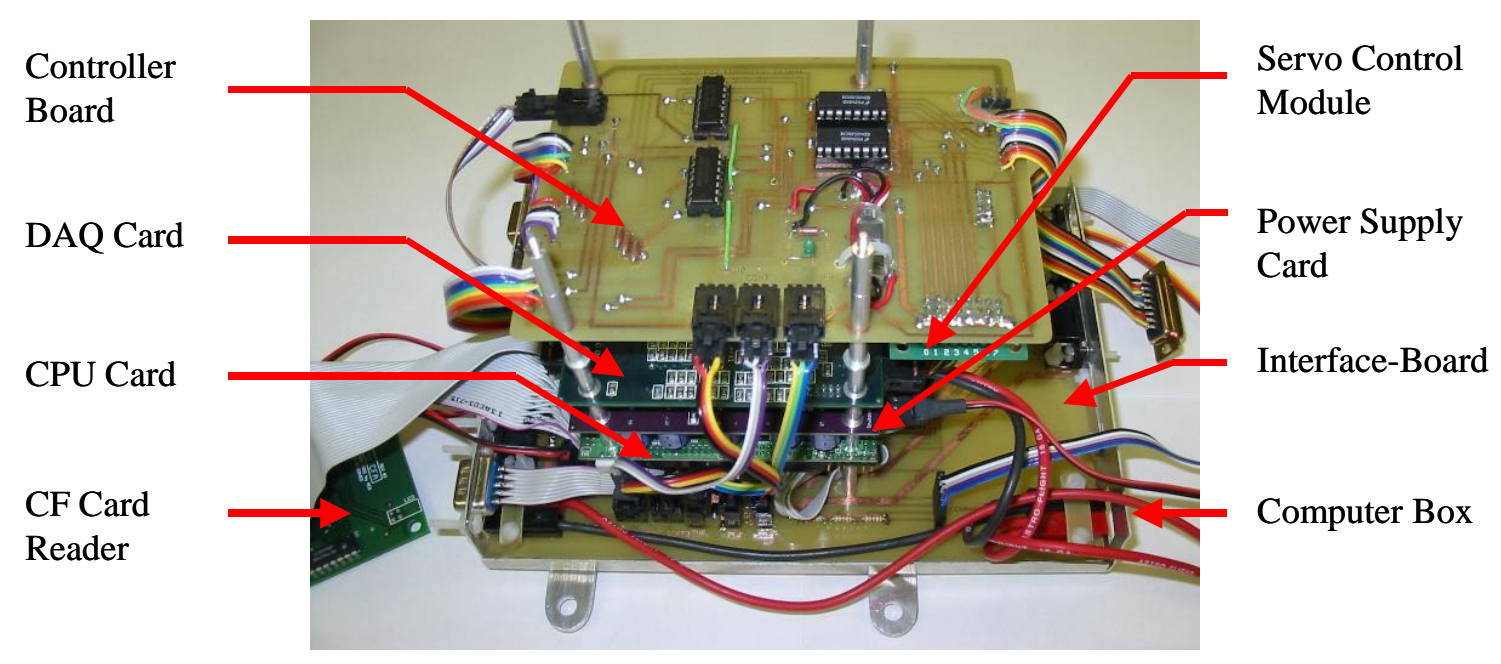

Figure 4-10 YF-22 On-board Computer

A description of each individual component that makes up the stack will now be discussed.

\section{CPU Module - MSI-CM588}

The CPU card is the "brain" of the aircraft payload. It collects data from the data acquisition card and executes the control laws. It sends out control commands to the controller board. The speed of the CPU was also a requirement since the designed AFA controller feature on-line learning neural networks. The CPU card that selected was the MSI-CM588 (Figure 4-11) manufactured by Microcomputer Systems.

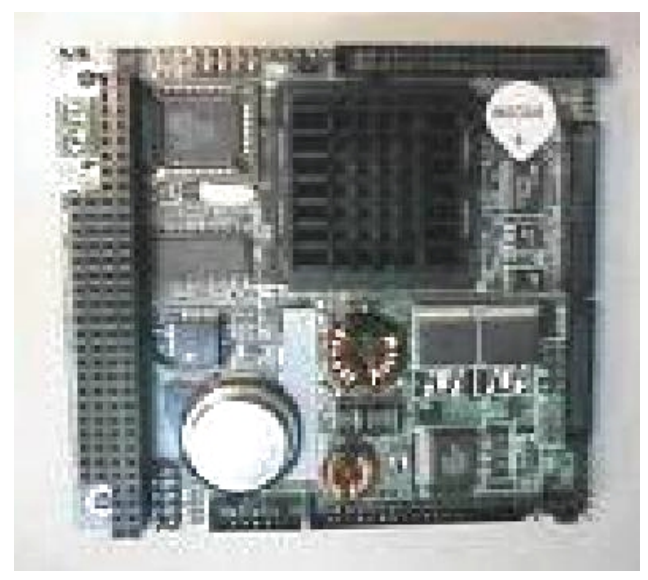

Figure 4-11 CPU Module

The MSI-CM588 is a versatile low-power PC/104 CPU card featuring a NS GXLV/GX1 processor and a GX5530 chipset with a built-in $6 \times 86300 \mathrm{MHz}$ CPU operating from 0 to 
$85^{\circ} \mathrm{C}$ without a fan from a single $+5 \mathrm{~V}$ power supply. The MSI-CM588 supports on-chip VGA display and two serial ports. $128 \mathrm{MB}$ memory had been installed on the CPU card for each on-board computer system.

\section{Data Acquisition Module - Diamond-MM-32-AT}

The data acquisition card is one of the most important modules in the system. Its main function is to collect signals from the individual sensors; furthermore, it sends channel selection commands to servo driving circuit through the digital output capability. The accuracy of the flight control command was directly dependant on the speed and accuracy of the data acquisition card. The data acquisition card selected was a DiamondMM-32-AT (Figure 4-12) made by Diamond Systems.

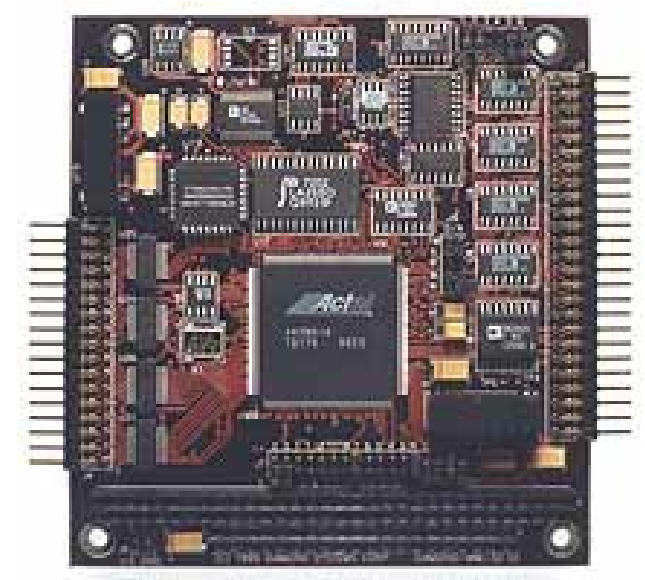

Figure 4-12 Data Acquisition Module

It has 32 analog input channels with 16 bits resolution. The maximum sampling rate is $200 \mathrm{KHz}$ (although the on-board computer system only uses up to $100 \mathrm{~Hz}$ ). The card is also capable of providing 24 high-current digital I/O; eight of them were used to send the channel selection signal to the controller board.

\section{Power Supply and Communication Module - Jupiter-MM-SIO}

The power supply card selected is the Jupiter-MM-SIO (Figure 4-13) manufactured by Diamond Systems. 


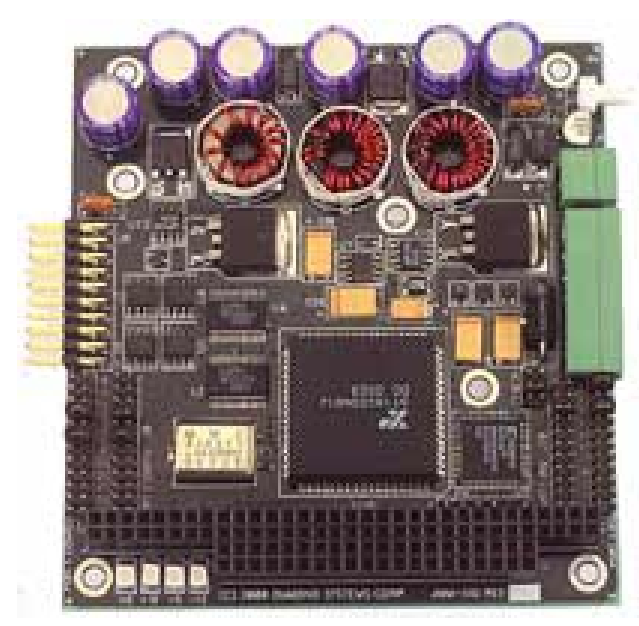

Figure 4-13 Power Supply Module

This module provides different voltage levels to power up the OBC and aircraft sensors. It also provides two additional serial ports, which can be used for communication and/or control purposes

\section{Servo Control Module}

The servo control module transfers the OBC's control commands (or serial signals) into the PWM signal to drive the aircraft servos. The servo control module used for the electronic payload is the Mini SSC II (Figure 4-14).

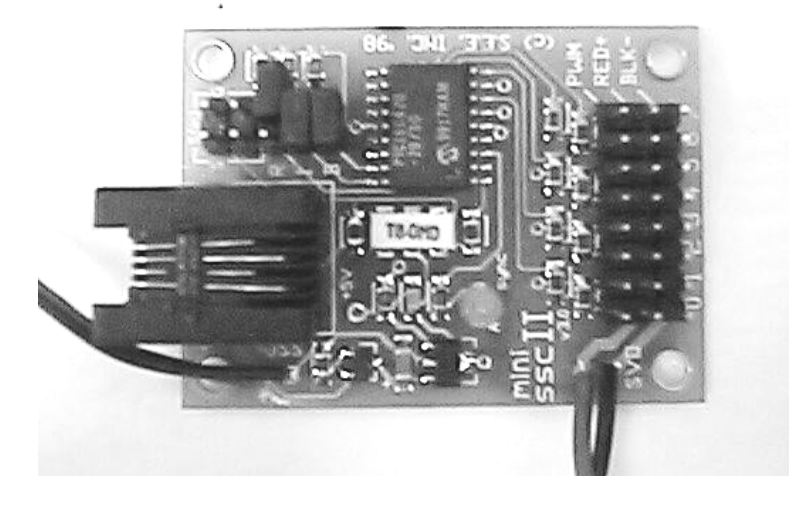

Figure 4-14 Servo Control Module

This module accepts serial inputs at 2400 or 9600 bps and provides 8 channels of servocontrol signals (PWM). It takes a three-byte control package including one header byte, one channel selection byte and one byte for control signal. In the YF-22 payload design, 
six controlling channels were used to control the aircraft with an update frequency of 50 $\mathrm{Hz}$.

\section{Compact Flash Reader}

Both the operating system and flight control software were installed in an $8 \mathrm{MB}$ removable compact flash card. An IDE card reader (Figure 4-15) had been installed on the flight computer.

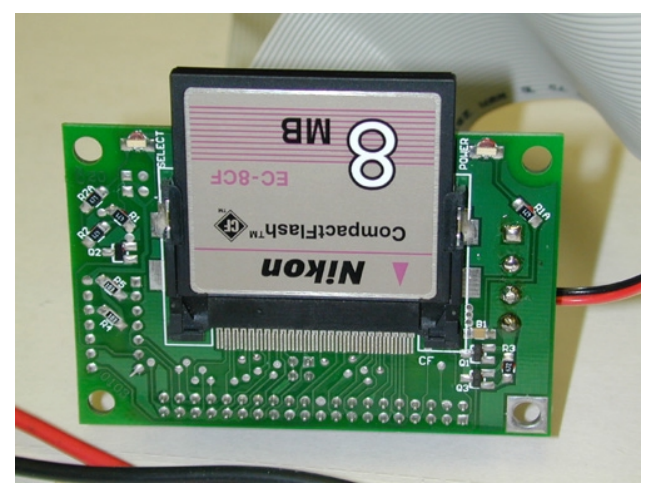

Figure 4-15 Compact Flash Card and Reader

This device is bootable and works like a hard drive. However, it can work within a much higher vibration environment. During flight testing sessions, different tasks can be stored in different compact flash cards for quick task reconfiguration at the field. The use of a removable compact flash card greatly also simplifies the procedure for data downloading after each flight.

\subsection{2 - On-board Sensors}

The on-board sensors provide measurements of all the main aircraft flight parameters that were used for both parameter identification and control purposes. A network of sensors for the aircraft model include: air data probe, pressure sensors, temperature sensor, IMU, vertical gyro, GPS and potentiometers for measure the deflection of each control surfaces. A detailed description of each component will now be provided.

\section{Nose Probe - SpaceAge ${ }^{\circledR}$ Mini Air Data Boom}

The nose probe (Figure 4-16) was manufactured by SpaceAge, Inc. 


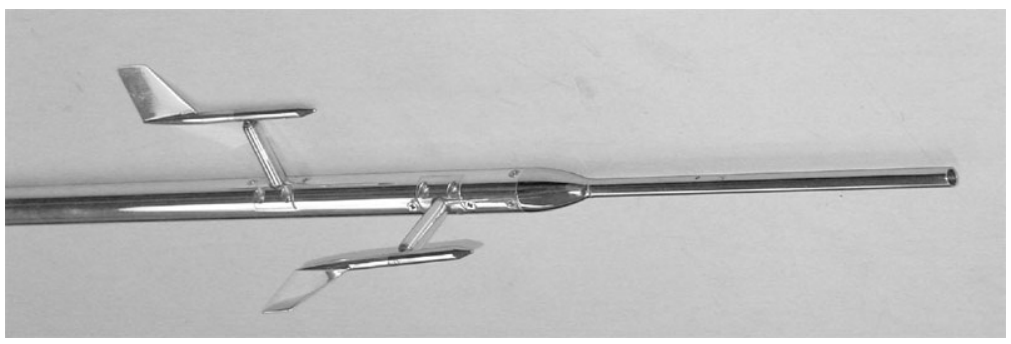

Figure 4-16 Air Data Probe

It is a lightweight component (approx. $6 \mathrm{oz}$ ) specially designed for light aircraft and UAV uses. The total length of the probe is 30 inches and the nominal maximum calibrated speed is 340 knots. It features angle-of-attack and sideslip vanes as well as static and dynamic pressure pickups.

\section{Pressure Sensors}

For measuring the dynamic and static pressure from the nose probe, two types of pressure sensors (Figure 4-17) were used:

$\begin{array}{ll}\text { Differential Pressure Sensor: } & \text { SenSym ASCX01DN } \\ \text { Absolute Pressure Sensor: } & \text { SenSym ASCX15AN }\end{array}$

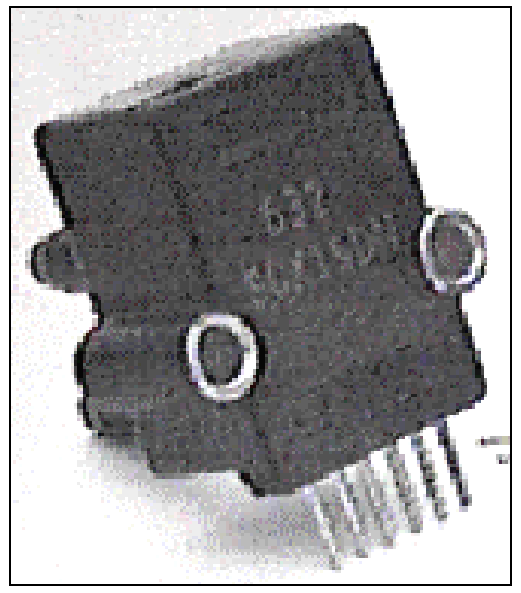

Figure 4-17 Pressure Sensor

The absolute pressure sensor measures the static pressure, which can be used to calculate the altitude of the UAV. The range of the SenSym ASCX15AN is 0-15 psi. The differential pressure sensor measures the difference between the static and dynamic pressure, which can be used to calculate the airspeed of the aircraft. The range of the SenSym ASCX01DN is 0-1 psi. 


\section{Inertial Measurement Unit (IMU) - IMU400}

The IMU selected for the YF-22 payload is the Crossbow IMU400 (Figure 4-18).

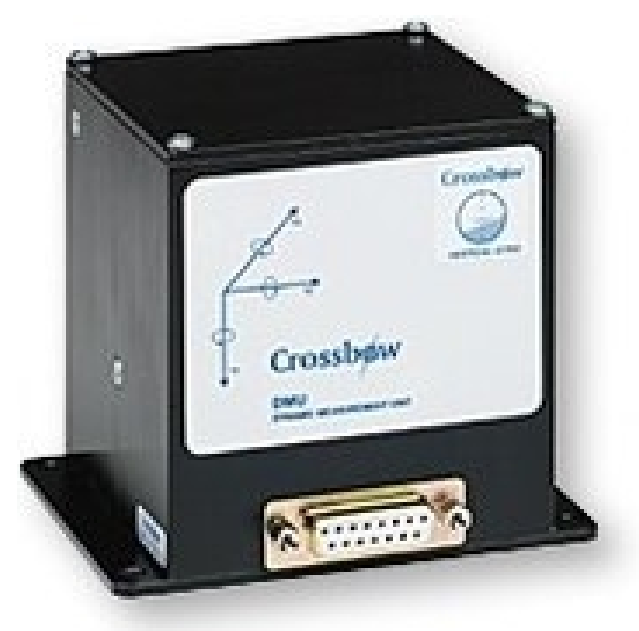

Figure 4-18 IMU400

This component is a solid-state six-degree-of-freedom (6 DOF) inertial package intended for navigation and control, dynamic testing, and instrumentation applications. This high reliability inertial system provides accurate measurements of angular rates and linear accelerations.

The IMU400 achieves excellent performance by employing proprietary algorithms to characterize and correct for the effects of temperature, linearity, and misalignment. Fully compensated angular rate and acceleration outputs are provided in both analog and digital (RS-232) formats. The range of measurement for the IMU400 unit is $\pm 90 \% \mathrm{sec}$ for angular rates and $\pm 4 \mathrm{~g}$ for accelerations.

\section{Vertical Gyro - VG34}

The VG34 vertical gyro (Figure 4-19) - manufactured by Goodrich Sensor Systems - was selected for the measurement of the Euler angles. This unit was specifically selected because of its small physical size and high performance. 


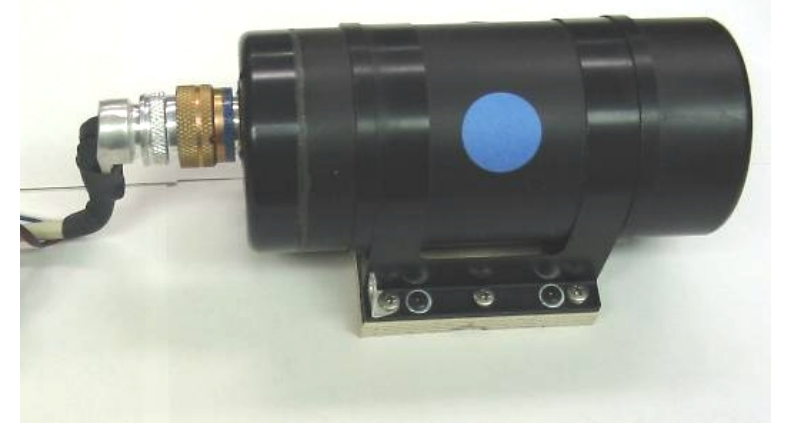

Figure 4-19 Vertical Gyro

The measurement range for the vertical gyro is $\pm 90^{\circ}$ in Roll, with an accuracy of $\pm 1^{\circ}$, and $\pm 60^{\circ}$ in pitch, also with an accuracy of $\pm 1^{\circ}$.

\section{Potentiometers}

To measure the displacement of aircraft's control surfaces, potentiometers (Figure 4-20) were installed on each axis.

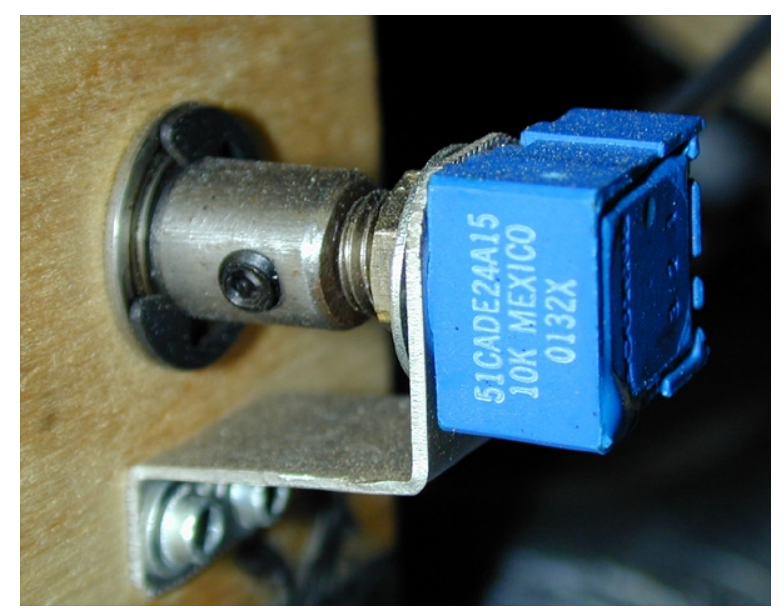

Figure 4-20 Potentiometer

The value of each potentiometer was selected to be $10 \mathrm{k} \Omega$. This value provided a desirable trade-off between Signal Noise ratio $(\mathrm{S} / \mathrm{N})$ and power consumption. 12 volts are supplied to each potentiometer and the reading output is collected and calibrated to provide the measurement of surface deflection in degrees. 


\section{GPS Unit}

The position and velocity information are not strictly required. However, they are helpful to verify other sensor data and assisted in obtain an accurate aircraft mathematic model. The GPS choice for the payload is the OEM4 (Figure 4-21) GPS unit made by Novatel, Inc.

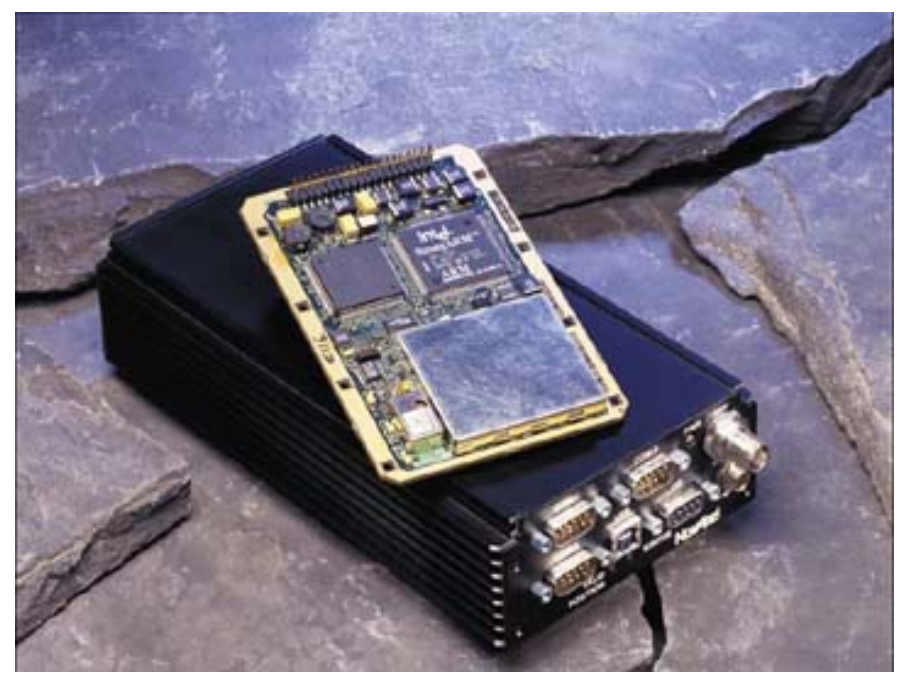

Figure 4-21 GPS Unit

Key benefits of the OEM4 unit include the following: Up to $20 \mathrm{~Hz}$ data update rate; Pulse Aperture Correlation (PAC) technology offers significant multipath elimination capabilities; On-board power conversion eliminates the need for external power conditioning; on-board voltage and temperature monitoring provide greater system reliability. This GPS unit provides the 3-axis position and velocity information of the aircraft via the serial port. The GPS antenna used on the YF-22 research UAV is the GPS-511 made by Novatel. A picture of the antenna unit mounted on top of the aircraft is shown in Figure 4-22. 


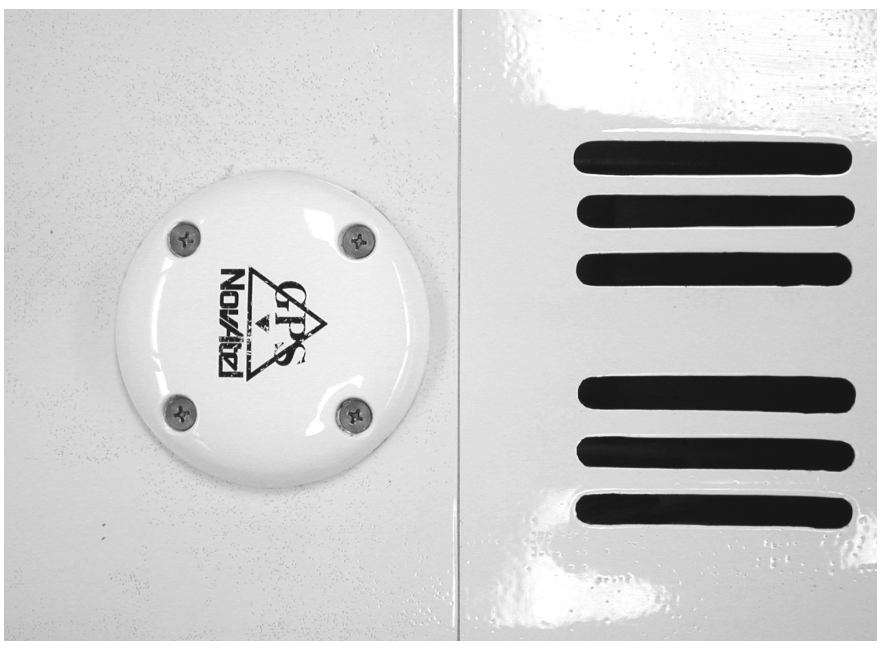

Figure 4-22 GPS Antenna

The GPS-511 offers optimal L1 performance for airborne and high dynamic applications. Along with a low profile, the GPS-511 is just 89 millimeters in diameter, weighing 145 grams, and is environmentally sealed to protect against harsh weather. The antenna features a four-hole mounting system to ensure secure installation.

\subsection{3 - Custom Designed Components:}

In addition to off-shelf components, several pieces of hardware had to be customdesigned from scratch to make the system fully functional. Printed Circuit Boards (PCB) had been designed and developed to meet the specified requirements including a baseboard, controller board, nose sensor board, power supply board, sensor \& servo hub boards.

\section{Baseboard}

The baseboard shown in Figure 4-23 is a custom-made signal connection board designed to connect individual sensor outputs to each specified data acquisition channel. It provides power to each sensor (except vertical gyro and GPS which are powered separately) and provides a reference voltage for the controller usage. 


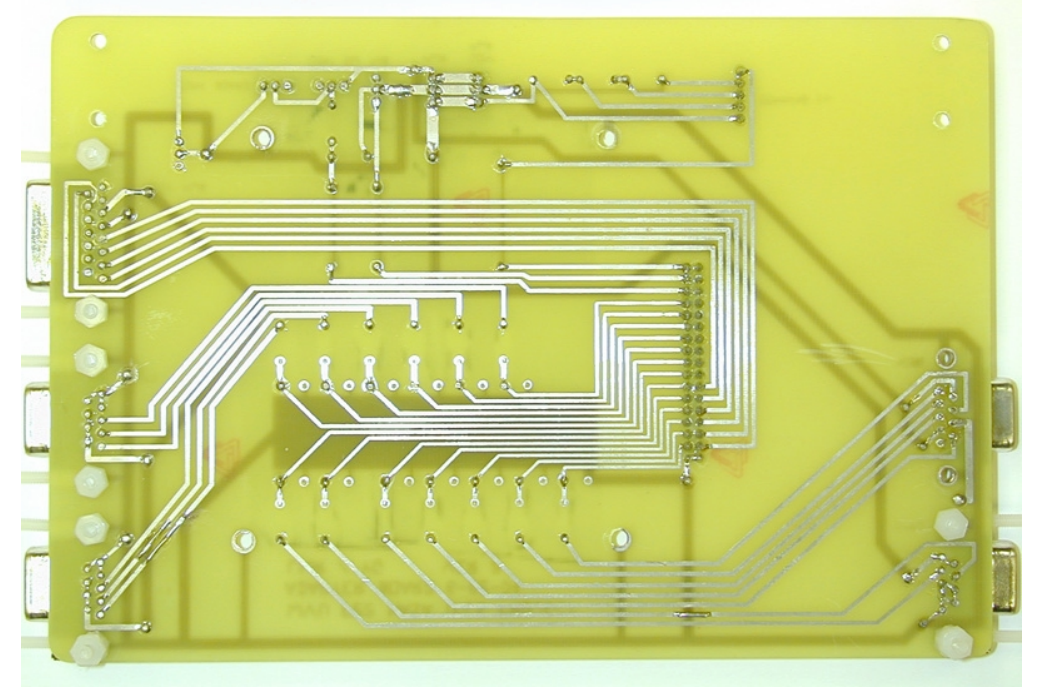

Figure 4-23 Baseboard

\section{Controller Board}

The controller board shown in Figure 4-24 below is one of the most critical components of the flight control hardware.

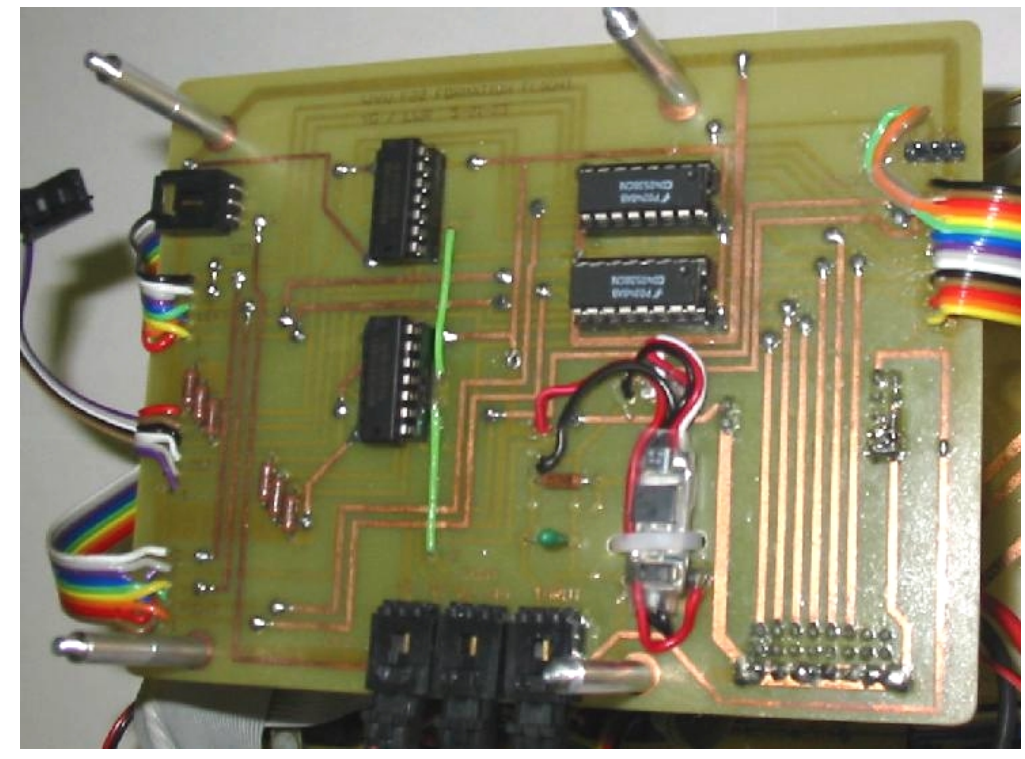

Figure 4-24 Controller Board

In fact, the safety of the aircraft is directly related with the reliability of the controller board. This board receives control signals from both the pilot $(\mathrm{R} / \mathrm{C}$ receiver) and the OBC (which then converts to PWM signal). Two switching mechanisms had been designed to guarantee the safety that is: Hardware Switching and Software Switching. 
Hardware Switching: Hardware switching gives the pilot the ability to switch off the entire controller instantly at any circumstance even if the on-board computer power is lost. A $\mathrm{R} / \mathrm{C}$ channel was allocated for this exact purpose. The PWM controller switch signal from the receiver has been converted to High/Low switching signal according to the signal pulse width. This switching signal is then used to drive a set of AND gates to enable/disable the entire on-board controller.

Software Switching: Software Switching gives the on-board computer the capability to control all or any subset of the aircraft's control surfaces with preprogrammed selections. With this ability, the flight test can be configured to contain different subtests and greatly enhances the flexibility and the safety of the experiment. The software switching is a cooperation of both hardware and software. The on-board software reads pre-determined channel selection information from a log file at the initialization stage. Once the controller switch is turned on, it sends out the channel selection signal through the Digital Input/Output (DIO) port of the data acquisition card. This signal is passed to the multi-channel 74HC4053 analog multiplexer/demultiplexer on the controller board to select the pilot/on-board control. The design of the controller board is shown in Figure 4-25.

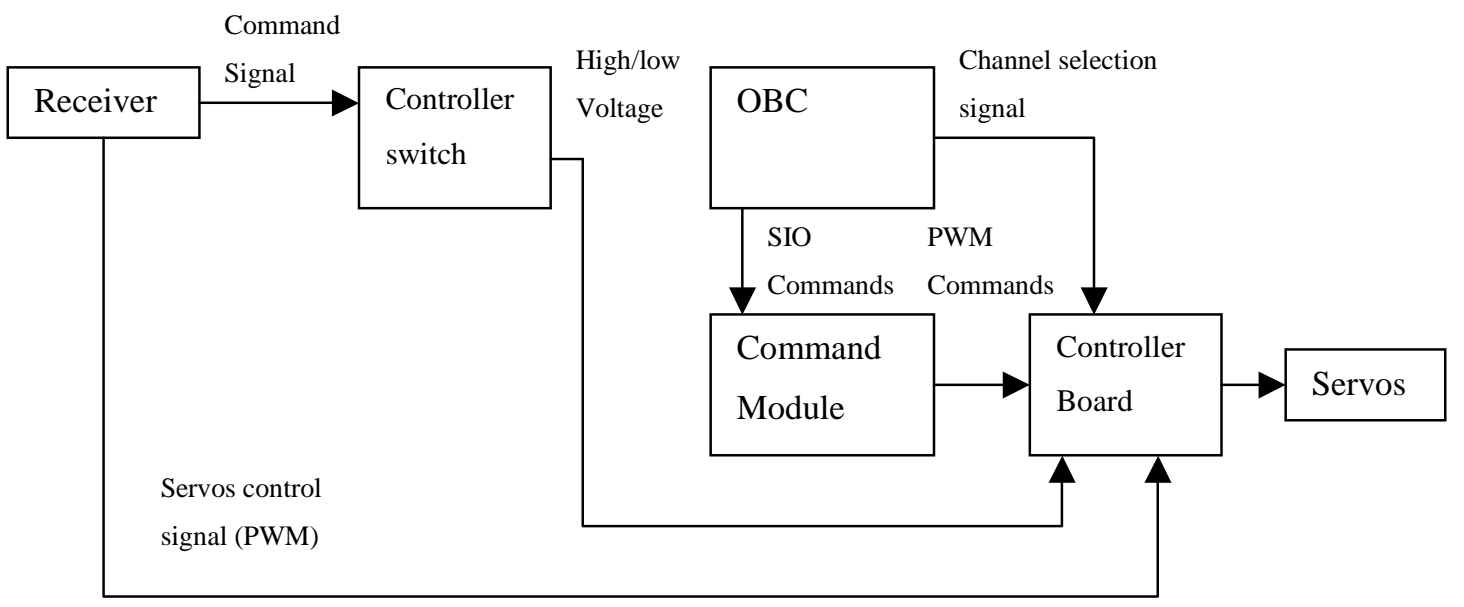

Figure 4-25 Controller Board Design 


\section{Nose Sensor Board}

The nose sensor board (Figure 4-26) was designed to interface with the dynamic pressure sensor, static pressure sensor and temperature sensor.

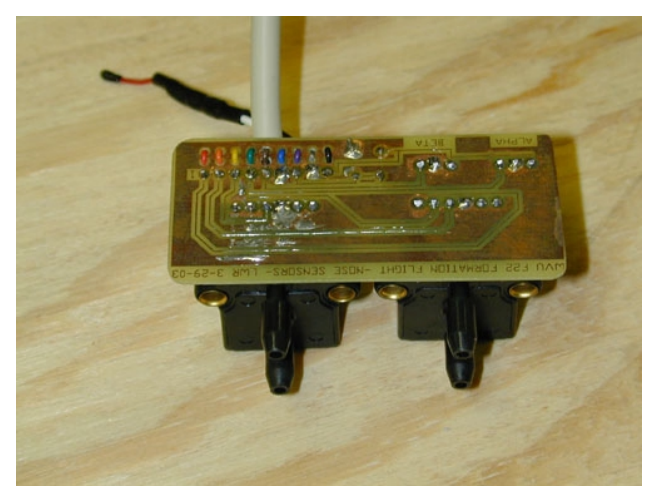

Figure 4-26 Nose Sensor Board

Additional connectors on the nose-board allow for the air-data probe potentiometers to be added to the data acquisition system.

\section{Power Supply}

The voltage requirement of the vertical gyro is a $24-32 \mathrm{~V}$ supply-range. The aircraft on-board battery pack only supplies $14.8 \mathrm{~V}$; therefore, a DC-DC converter was necessary. The $24 \mathrm{v}$ DC converter was mounted on a custom-made power supply PC board (Figure 4-27).

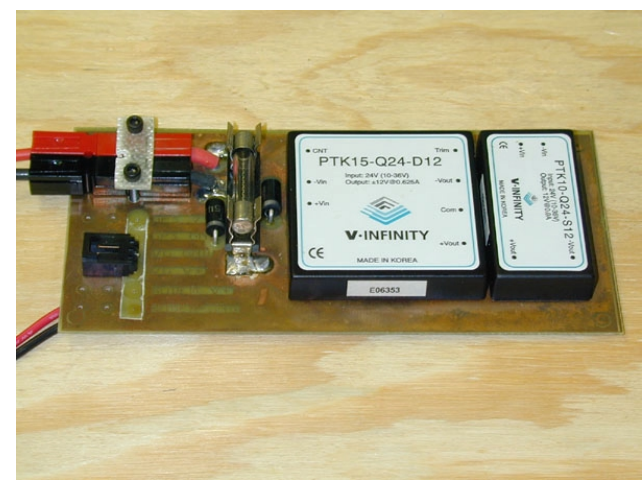

Figure 4-27 Power Supply

To minimize potential EMI problems several RF chokes were used in-line and the package is enclosed inside an aluminum case. 


\section{Sensor Hubs}

The sensor hub (Figure 4-28) was designed to connect the on-board computer to the potentiometers mounted on each control surface.

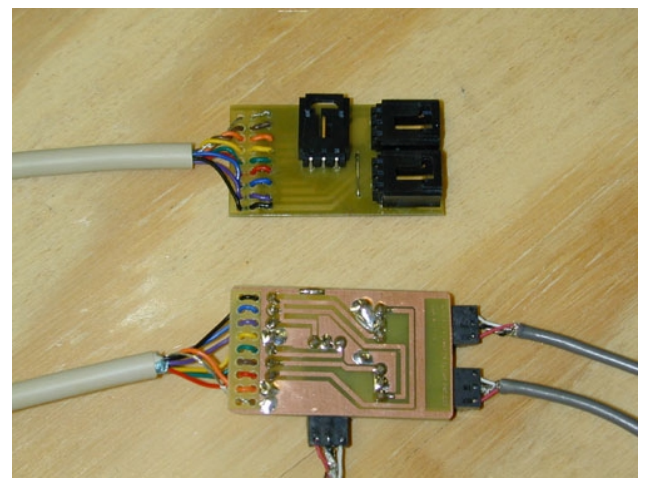

Figure 4-28 Sensor Hub

12 volts had been supplied to each of the potentiometer and the signal reading is passed back to the on-board data acquisition card. Two sensor hubs, located near aircraft surfaces, were used to provide connections on the left and right sides of the plane.

\section{Servo Hubs}

The servo hub was designed to connect the controller hardware to individual servos (Figure 4-29).

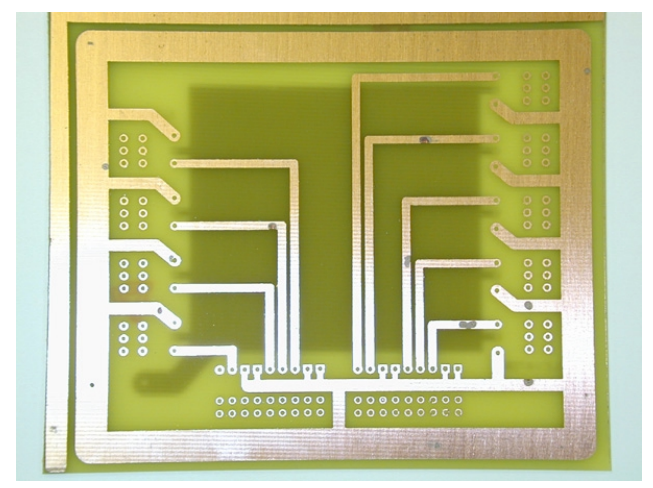

Figure 4-29 Servo Hub

The servo control commands were sent out to the servo hub and then re-distributed to each individual servo including left/right elevators, left/right rudders, left/right ailerons, left/right flaps, and the engine throttle signal. 


\section{Interface Panel}

The interface panel is integrated on the computer enclosure. It is made of aluminum and features power and mode switches and provides connection to sensors, monitor, and keyboard. The interface panel has three sections: front, rear and top (Figure 4-30).

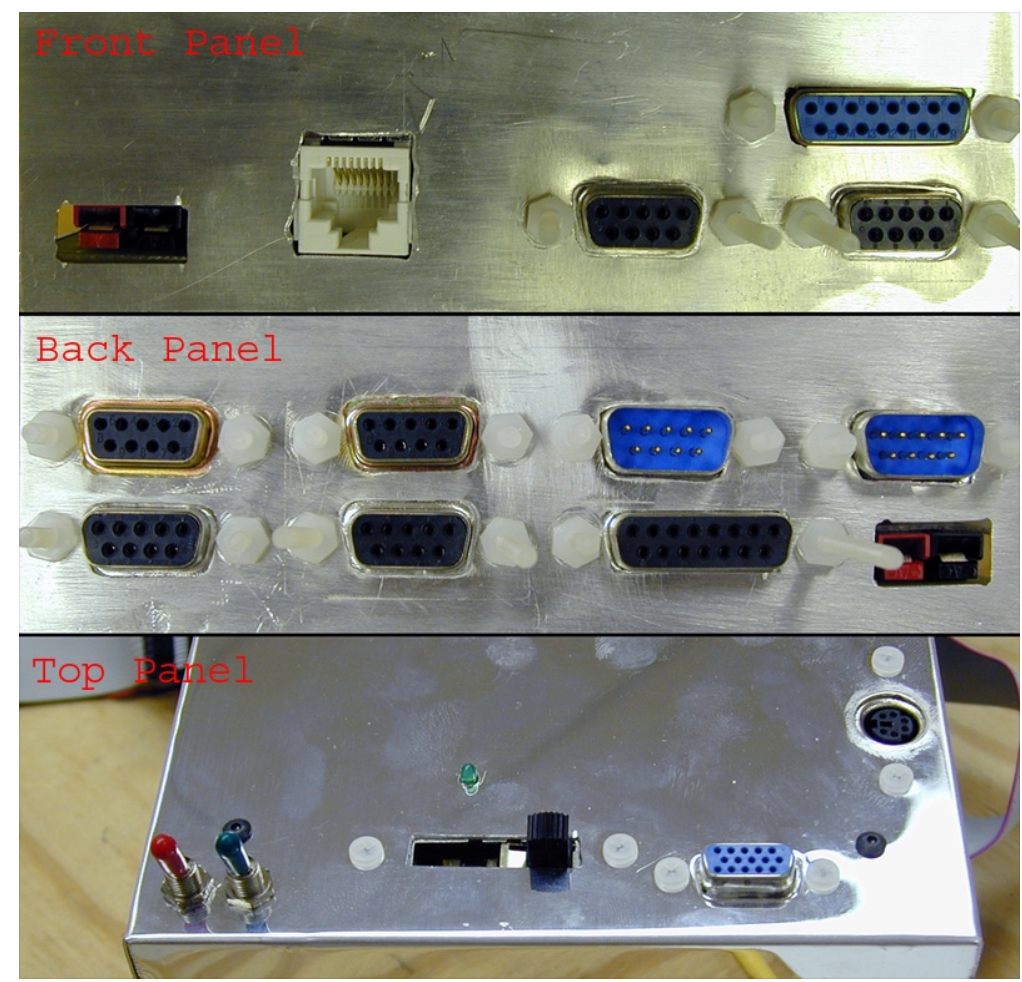

Figure 4-30 Interface Panels

The front panel connects to the battery power, $\mathrm{R} / \mathrm{C}$ receiver; vertical gyro and nose probe sensors. The rear panel connects to the IMU, sensor hubs, servo hub, and GPS. The top panel is for human interface and contains a computer power switch, vertical gyro power switch, running mode switch, power LED, controller switch LED, and a slot for the compact flash card.

\subsection{4 - Power Sources}

A total of six battery packs have been used in each WVU research UAV. Four $4.8 \mathrm{v} 1600 \mathrm{mAh}$ NiMN battery packs were used for R/C system including two for the receiver and two for the aircraft servos. This provided a dual-redundancy for the power 
of the R/C system, which is the most critical part of the aircraft safety. A $7.2 \mathrm{v} 1250 \mathrm{mAh}$ $\mathrm{NiCd}$ battery pack had been used to power the electronics for the jet engine. To power the on-board computer and instruments, a battery pack made of four Li-Poly battery cells (Figure 4-31) was used.

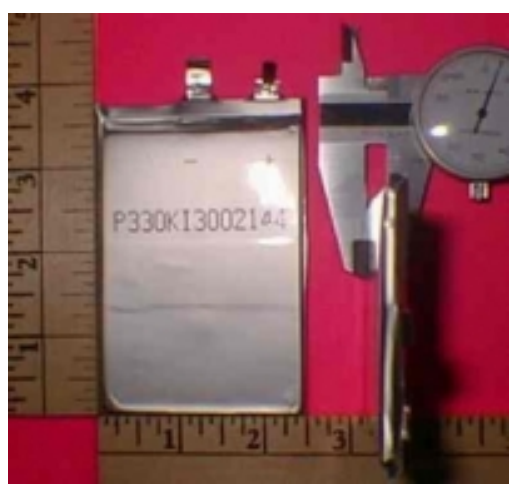

Figure 4-31 Battery Cell

This battery pack provided 14.8v (nominal) with 3300mAh capacity. The YF-22 onboard payload power consumption in Table 4-2 shows that the selected Li-Poly battery pack can last for more than one hour after been fully charged, which is more than adequate for AFA flight testing purposes.

\begin{tabular}{|c|c|c|c|}
\hline Device & Current (start) & Current (norminal) & Notes \\
\hline Vertical Gyro & 1.0 & 0.5 & $\begin{array}{c}\text { Requires a 3-minute } \\
\text { stabilizaion phase }\end{array}$ \\
\hline OBC & 0.7 & 0.65 & Without sensor power \\
\hline GPS & 0.2 & 0.2 & Estimate \\
\hline Others & 0.5 & 0.5 & \\
\hline Total Estimation & 2.4 & 1.85 & \\
\hline
\end{tabular}

Table 4-2 On-board Payload Power consumption

\subsection{Hardware Mounting}

The installation of the hardware components needs carefully consider the following important factors:

- Characteristic of each instrument,

- Cable length, 
- Level of vibration,

- Power supply,

- Electromagnetic interference,

- Balance of the aircraft

Most of the payload components including the on-board computer, vertical gyro, IMU, GPS, Power supply were mounted on the two rails of the payload bay shown in Figure 432.

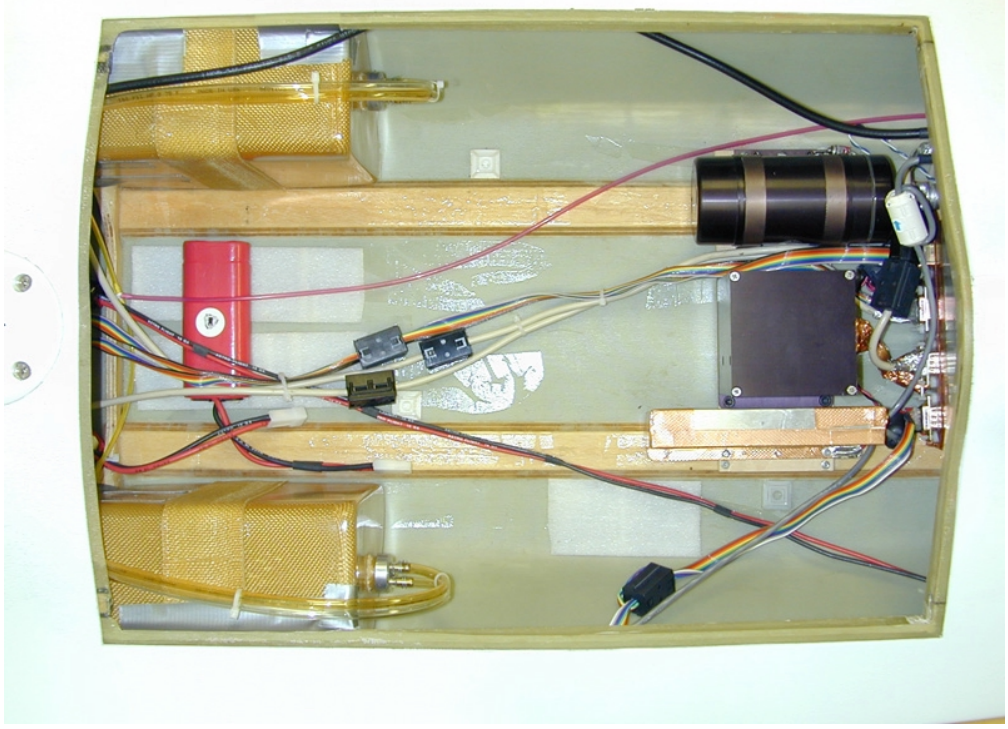

Figure 4-32 Payload Bay

With the jet engine mounted in the rear of the aircraft, most of the payload components were installed toward the forward section of the aircraft due to balance issues.

The ideal position of the IMU was the C.G. of the aircraft to provide the correct 3axis acceleration readings directly. However, because of balancing issues, the IMU had to be relocated to a position forward of the C.G. With this configuration, the acceleration read-outs on the $\mathrm{Y}$ and $\mathrm{Z}$-axis had to be corrected with the angular-rate readings. Since these two signals were not required in this project, the correction algorithms will not be discussed in this dissertation.

Both the vertical gyro and IMU needed to be perfectly leveled with the aircraft. The mounting of the nose probe was required to be parallel to the $\mathrm{X}$-axis of the aircraft. To be away from most EMI sources and use the maximum length of the aircraft for 
antenna, the $\mathrm{R} / \mathrm{C}$ receiver was mounted in the nose bay of the aircraft (Figure 4-33) with the antenna attached to the tip of right vertical stabilizer.

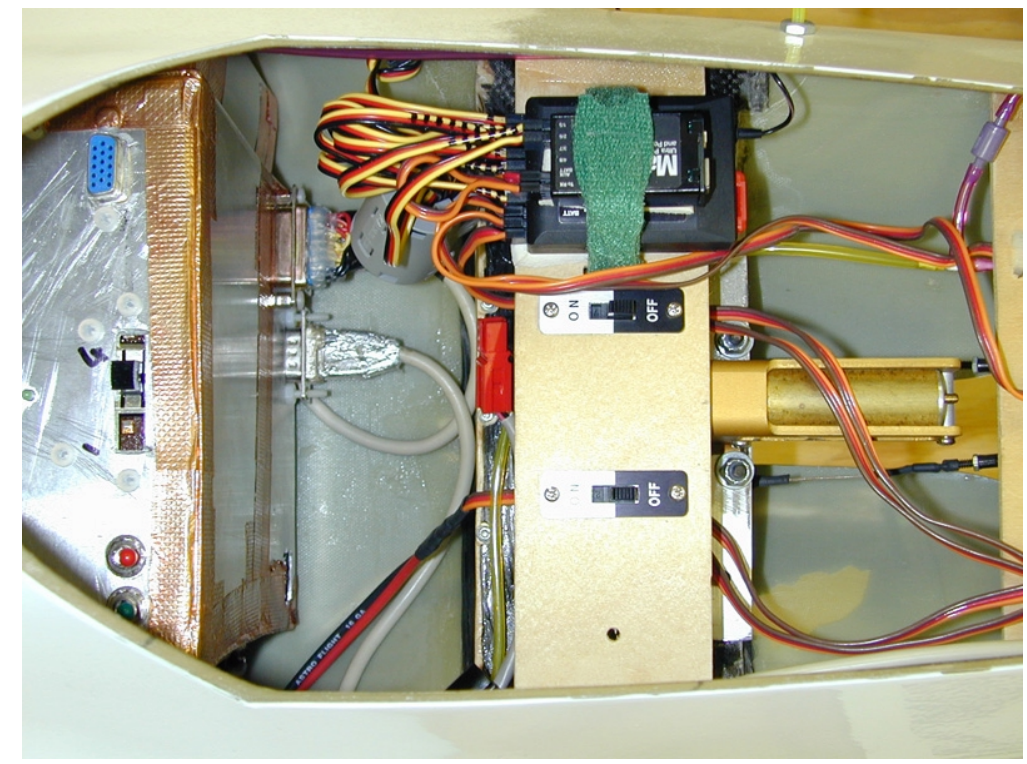

Figure 4-33 Nose Bay

\subsection{EMI}

ElectroMagnetic Interference (EMI) poses a significant threat for UAV safety. For a small UAV like the WVU YF-22 research aircraft model, there are several internal sources of interference including the on-board computer, vertical gyro, and power supply system. With the physical restriction of the aircraft, these components are located within a few feet of the $\mathrm{R} / \mathrm{C}$ receiver. Therefore, special care was taken in the design, manufacture and installation of each component. Aluminum enclosures were designed to shield most of the hardware components and ferrite RF chokes were used on every power and signal cables. The EMI of the completed on-board payload was evaluated with a spectrum analyzer before the first set of flight testing. To guarantee the safety of the aircraft, a ground range check for the $\mathrm{R} / \mathrm{C}$ radio system is performed before each flight. During the range check, the on-board computer, sensors and jet engine were powered and an approximate $300 \mathrm{ft}$ ground radio range was required with the transmitter antenna fully retracted before flight operations proceeded. 


\section{Chapter 5 On-board Software}

With the AFA controller been designed and all the on-board hardware been developed, software was required to implement the control laws and provide an interface between the controller and the on-board hardware. As for any flight control software, the on-board software must be executed in real time. Matlab ${ }^{\circledR}$ real-time workshop was selected to generate the real-time target. The software was designed to be modulized and each individual software component was programmed with C-language as a Matlab ${ }^{\circledR} \mathrm{S}$ functions. The Simulink ${ }^{\circledR}$ environment, a package within Matlab ${ }^{\circledR}$ was used as the simulation environment. Once simulated test was completed, a final program was compiled with Real-Time Workshop (RTW) as a real-time DOS target for flight testing. The operating system on-board the YF-22 flight computer system is DOS. The selection of DOS was due to its simplicity and limited storage requirement. Both the operating system and on-board software were stored on an $8 \mathrm{MB}$ compact flash card, which is a self-supporting bootable device. All of the software components are individual modules and are easy to be configured for various flight testing tasks.

The UAV on-board software was designed to perform data acquisition, execute control laws and implement the control commands. The following ten requirements had been used to define the software architecture:

1. Reliability;

2. Performance in Real Time;

3. System sampling rate no slower than $25 \mathrm{~Hz}$;

4. Data acquisition from all sensors and conversion into engineering units;

5. NN on-line training;

6. Execute control laws on-board;

7. Provide control command and control channel selection signals;

8. Store data for post flight analysis;

9. Ability to be reconfigured for different flight task at the flight testing facility (without recompiling);

10. Automatically update calibration data at field (without recompiling). 


\section{1 - Selected Architecture}

With the system requirements defined above, the YF-22 on-board software can be divided to be two major subsystems:

1. Data acquisition system;

2. AFA flight control system.

The data acquisition software acquires the sensor signals from analog I/O module, which converts the analog signal into a 16 bit digital signal. The raw voltage data is then calibrated on-board to generate meaningful engineering values to be used by the flight controller. The flight data is also saved in the $8 \mathrm{MB}$ on-board flash card for post flight analysis.

The AFA flight control software receives as input the flight data acquired through the DAQ software. On-line training NN based AFA controllers designed in Chapter 3 are executed in real-time. Commands generated by the control laws are then calibrated and sent to the servo control hardware for AFA purpose. A diagram of the on-board software is shown in Figure 5-1.

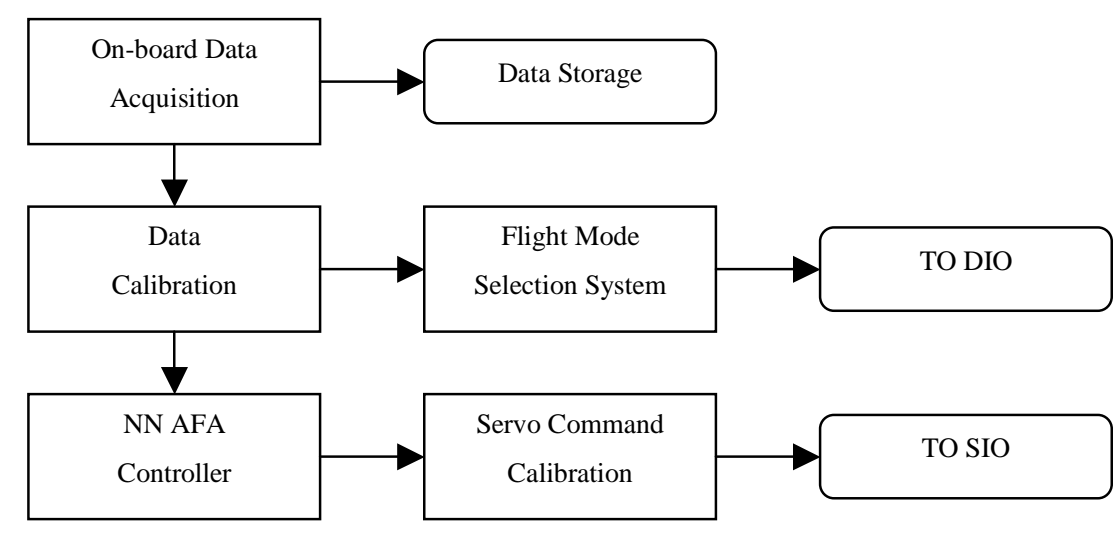

Figure 5-1 On-Board Software

\section{2 - Data Acquisition Software}

The main purpose of the data acquisition software is to collect, convert, send, and store sensor readings from the electronic payload. The development of this software was based upon a Diamond-MM-32 PC/104 format 16-bit analog I/O module. 


\section{Analog Input Channels:}

The Diamond-MM-32 features 32 analog I/O channels. Only 22 analog I/O channels were actually used. A list of the data acquisition channels is provided in Table $5-1$.

\begin{tabular}{|c|c|c|}
\hline Number & Channel Name & Sensor/Notes \\
\hline 1 & Static Pressure & Nose probe \\
\hline 2 & Dynamic Pressure & Nose probe \\
\hline 3 & Alpha & Nose probe \\
\hline 4 & Beta & Nose probe \\
\hline 5 & Temperature & Temperature sensor \\
\hline 6 & Roll Angle & Vertical Gyro \\
\hline 7 & Pitch Angle & Vertical Gyro \\
\hline 8 & Left Aileron & Potentiometer \\
\hline 9 & Left Rudder & Potentiometer \\
\hline 10 & Left Elevator & Potentiometer \\
\hline 11 & Right Aileron & Potentiometer \\
\hline 12 & Right Rudder & Potentiometer \\
\hline 13 & Right Elevator & Potentiometer \\
\hline 14 & Control Switch & Manual/Automatic control \\
\hline 15 & Throttle Reading & Receiver \\
\hline 16 & Command Switch & Voltage reference \\
\hline 17 & Acceleration_X & IMU \\
\hline 18 & Acceleration_Y & IMU \\
\hline 19 & Acceleration_Z & IMU \\
\hline 20 & $\mathrm{P}$ & IMU \\
\hline 21 & Q & IMU \\
\hline 22 & $\mathrm{R}$ & IMU \\
\hline
\end{tabular}

Table 5-1 Data Acquisition Channels 


\section{Input Ranges and Resolution}

All sensors in the electronic payload have an output range between -10 and $10 \mathrm{~V}$. Therefore all the analog I/O channels were configured to accept $\pm 10 \mathrm{~V}$ bipolar inputs. With the 16 bits $\mathrm{A} / \mathrm{D}$ conversion, the resolution of the data acquisition is $305 \mu \mathrm{V}$, which is accurate enough for data analysis and control purposes.

\section{A/D Conversion Formulas}

The 16-bit value returned by the A/D converter is always a complement number ranging from -32768 to 32767 , regardless of the input range. The input signal is actually magnified and shifted to match this range before it reaches the A/D. The A/D conversion formula for bipolar input range is:

$\mathrm{FS}=$ full-scale voltage (e.g. 10 for $\pm 10 \mathrm{~V}$ range $)$

Input voltage $=(\mathrm{A} / \mathrm{D}$ code $/ 32768) \times \mathrm{FS}$

\section{A/D Conversion}

There are 7 steps involved in performing this A/D conversion [73]:

\section{Step \#1: Selection of the input channel or input channel range}

The Diamond-MM-32 contains a channel counter circuit that controls which channel is to be sampled on each A/D conversion command. This circuit uses two channel numbers called the low channel and high channel, which are stored in registers. The circuit starts at the low channel and automatically increments after each $\mathrm{A} / \mathrm{D}$ conversion until the high channel is reached. When an $\mathrm{A} / \mathrm{D}$ conversion is performed on the high channel, the circuit resets to the low channel and starts over again.

For the data acquisition software, the low channel was 0 and the high channel was 21 . The data acquisition card scans the whole range of 22 channels once for each sampling time. This range can also easily be expended as more sensors are added. 
Step \#2: Selection of the analog input range (range, polarity, and gain codes)

The desired input range can be selected by writing to the analog $\mathrm{I} / \mathrm{O}$ control register. The analog input range used by the on-board payload was $\pm 10 \mathrm{~V}$.

\section{Step \#3: Wait for analog input circuit to settle}

After changing either the input channel or input range, the circuit needs to settle on a new value before performing the A/D conversion. The settling time is substantial when compared with the software execution times. Therefore, a timer was provided on board to indicate when it is safe to precede with the A/D sampling. The WAIT bit indicates when the circuit is settling and when it is safe to sample the input.

\section{Step \#4: Start an A/D conversion on the current channel}

To generate an A/D conversion, write to base address to start the conversion.

\section{Step \#5: Wait for the conversion to finish}

The A/D converter takes about four microseconds to complete a conversion. The A/D converter provides a status signal to indicate whether it is busy or idle.

\section{Step \#6: Read the A/D data}

Once the conversion is completed, the data can be read back from the A/D converter. The data is 16 bits wide and is read back in two 8-bit bytes: Least Significant Byte (LSB) and Most Significant Byte (MSB). The following pseudocode illustrates how to construct the 16-bit A/D value from these two bytes:

$$
\begin{array}{ll}
\text { LSB }=\text { read (base) } ; & \text { Get low } 8 \text { bits } \\
\text { MSB }=\text { read (base }+1) ; & \text { Get high } 8 \text { bits } \\
\text { Data }=\text { MSB * 256 + LSB } ; & \text { Combine the } 2 \text { bytes into a 16-bit value }
\end{array}
$$


The final data ranges from 0 to $65535\left(0\right.$ to $\left.2^{16}-1\right)$ as an unsigned integer. This value must be interpreted as a signed integer ranging from -32768 to +32767 .

\section{Step \#7: Convert the numerical data to meaningful engineering unit}

Once the data acquisition is completed, it only provides a voltage reading of the individual sensor. For use by the controller board, the voltage value needs to be converted to an engineering unit. The calibration information for each channel was calculated and stored on the compact flash card. The on-board software loads the calibration file at the initialization phase of execution and converts the data at each time step before feeding the results into the controller. A block diagram for the on-board Data Acquisition Software is shown in Figure 5-2. 


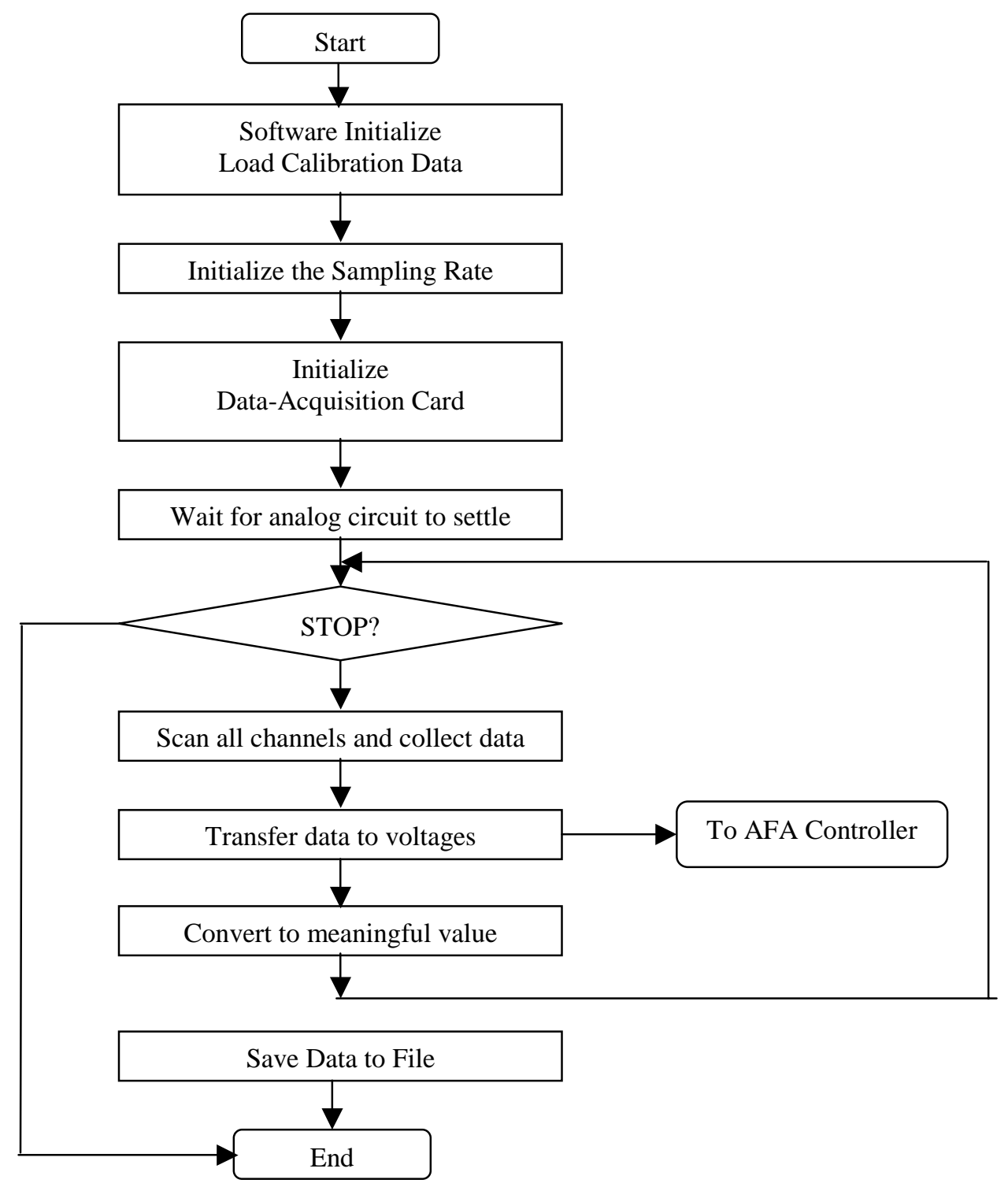

Figure 5-2 Data Acquisition Software

\section{Digital Input Channels}

The 3-axis position and velocity information of the aircraft GPS unit was acquired on-board through the serial port. A total of six channels were acquired, which are shown in Table 5-2 


\begin{tabular}{|l|l|}
\hline Number & Channel Name \\
\hline 1 & Position $-\mathrm{x}$ \\
\hline 2 & Position $-\mathrm{y}$ \\
\hline 3 & Position $-\mathrm{z}$ \\
\hline 4 & Velocity - $\mathrm{x}$ \\
\hline 5 & Velocity - y \\
\hline 6 & Velocity - $\mathrm{z}$ \\
\hline \multicolumn{2}{|c|}{ Table 5-2 GPS Channels }
\end{tabular}

The controller command sent by the on-board computer was also recorded for post-flight analysis. A description of controller command channels is shown in Table 5-3

\begin{tabular}{|l|l|}
\hline Number & Controller Command \\
\hline 1 & Left Elevator \\
\hline 2 & Right Elevator \\
\hline 3 & Left Aileron \\
\hline 4 & Right Aileron \\
\hline 5 & Left/Right Rudders \\
\hline 6 & Engine Throttle \\
\hline
\end{tabular}

Table 5-3 Control Command Channels

\section{Sampling Rate}

The sampling rate of the data acquisition software is adjustable. Due to the fast dynamics of the small UAV, the sampling rate of the on-board analog DAQ should be no slower than $25 \mathrm{~Hz}$. For flight tests for data-acquisition purposes - such as the PID phase of the program - the sampling rate was set to be $100 \mathrm{~Hz}$. For flight testing with flight control system and AFA software, the sampling rate was reduced to $50 \mathrm{~Hz}$ to save on computation power. The overall sampling rate for the GPS unit was $20 \mathrm{~Hz}$, which is the maximum allowed by the selected hardware. 


\section{Data Outputs}

The flight data acquired with the DAQ software can be used for three different purposes:

1. Pass to the on-board AFA flight control software simultaneously for controller use.

2. Send out selected sensor readings (reduced sampling rate) through a serial port simultaneously. During flight tests, this signal can then be received by the ground station through optional RF-Modems.

3. Save into a data file for post flight download and analysis by engineers.

\section{3 - AFA Flight Control Software}

The AFA Flight Control software gives the on-board computer the capability to control the aircraft and accommodate for the actuator failure. In addition to the data acquisition software, several additional components are necessary to meet this goal:

- Digital channel selections;

- AFA controller;

- On-board servo calibration;

- Servo control.

A Simulink diagram for actuator failure accommodation flight control software is shown in Figure 5-3. This scheme was used for the final AFA flight testing. 


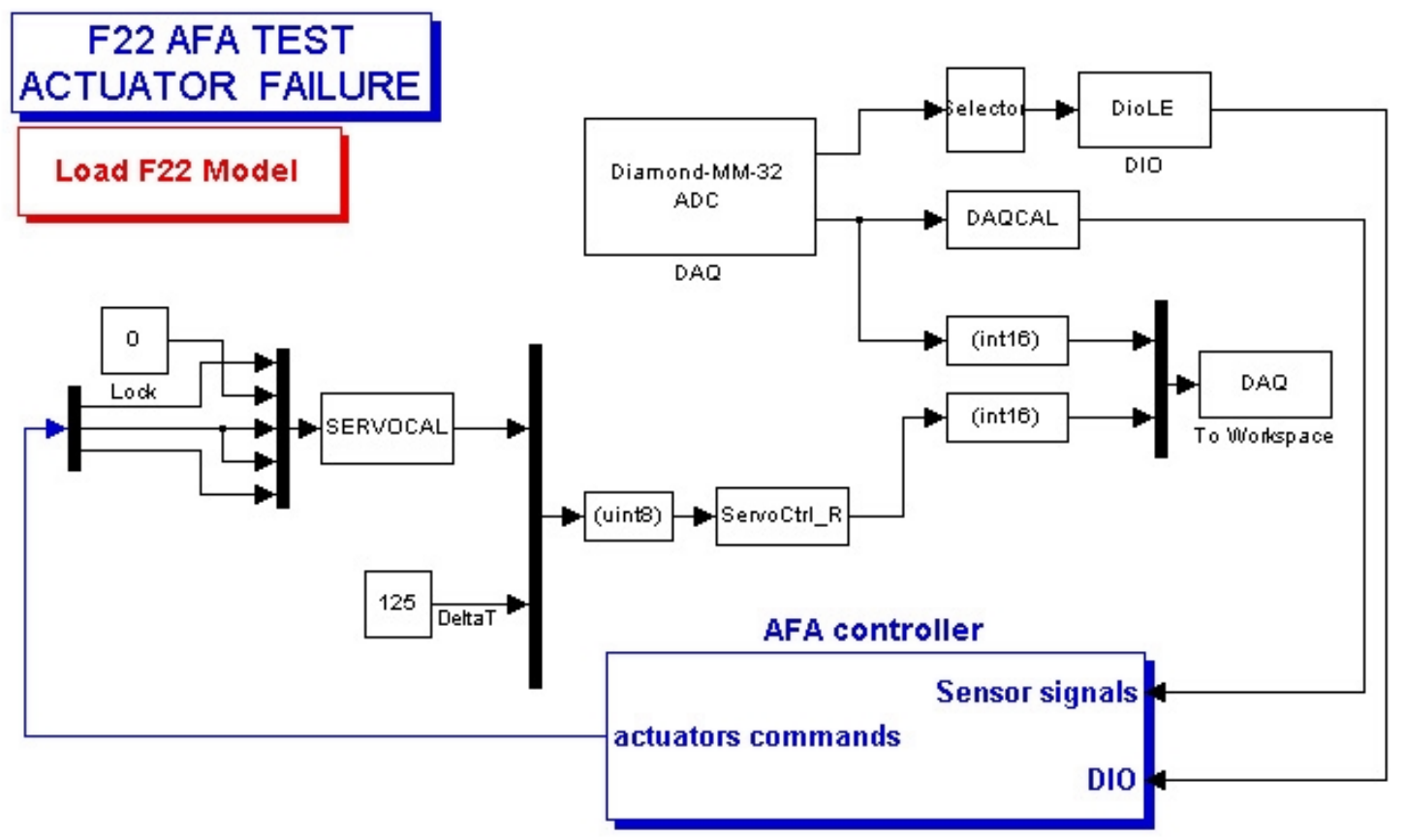

Figure 5-3 Simulink Diagram for On-Board AFA Software

\section{Digital Channel Selection}

The YF-22 on-board payload has the ability to be configured for different flight testing tasks on the field. To simplify the problem and for safety purposes, during some flight-tests only a subset of control surfaces were required to be controlled by the on-board computer while the other channels remained in full control of the pilot. A digital channel selection module was necessary in the flight control software to perform this selection of the channels.

A small data file called "Judgenum.dat" was stored on the computer's compact flash card. This file provided a six-digit binary number deciding on which channel was selected. A relationship between the number and control activity is shown in Table 5-4. The digital channel selection software reads this number at the initialization stage of the execution and saves into memory. In this way, different flight tests using a different subset of control surfaces may be configured during a flight session in the field without recompiling the on-board software. 


\begin{tabular}{|c|c|l|}
\hline Binary Number & Decimal Number & Control Activity \\
\hline 000000 & 00 & No Control \\
\hline 000001 & 01 & Left Elevator \\
\hline 000010 & 02 & Right Elevator \\
\hline 000011 & 03 & All Longitudinal Surfaces \\
\hline 000100 & 04 & Left Aileron \\
\hline 001000 & 08 & Right Aileron \\
\hline 001100 & 12 & Both Ailerons \\
\hline 010000 & 16 & Rudders \\
\hline 011100 & 28 & All Lateral-Directional Surfaces \\
\hline 011111 & 31 & All Control Surfaces \\
\hline 100000 & 32 & Engine Throttle \\
\hline 111111 & 63 & All Control Channels \\
\hline
\end{tabular}

Table 5-4 Control Channel Selections

During the flight test, the digital channel selection software reads the control switch signal (channel 14) from the DAQ software. Once channel 14 is high $(5 \mathrm{~V})$, implying that the pilot turned the controller switch on, the digital channel selection will then start sending channel selection signal through the Digital Input/Output (DIO) port of the data acquisition card to the controller board. In this case, the channel pre-assigned will be activated and controlled by the on-board computer while the pilot retains control of all other channels.

\section{AFA Controller}

The structure of the AFA controller has been described with details in Chapter 3. For the case of aileron failure, the control scheme in Figure 5-4 was used and the control scheme in Figure 5-5 was designed to compensate the elevator failure. 


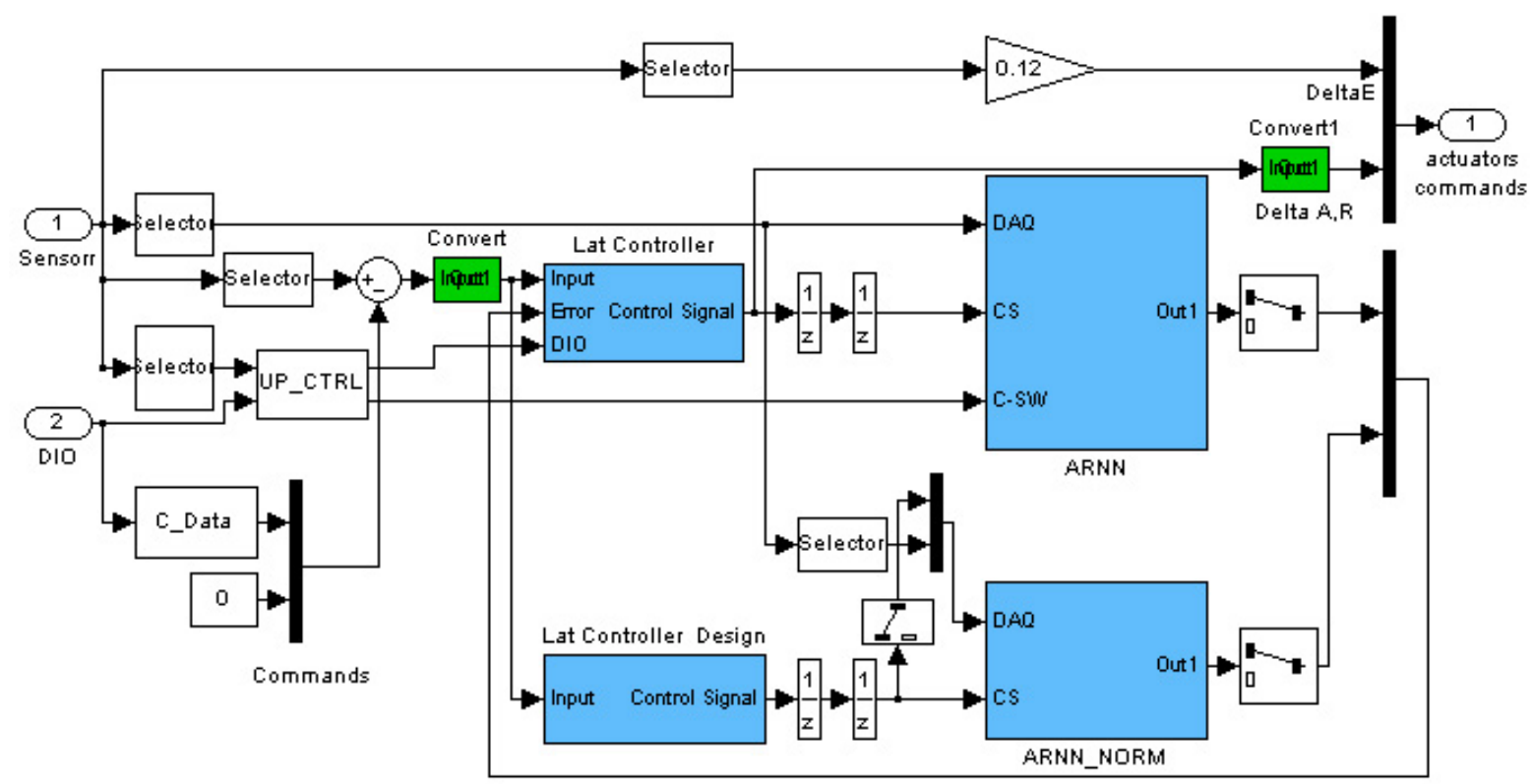

Figure 5-4 Simulink Diagram for Aileron AFA Controller

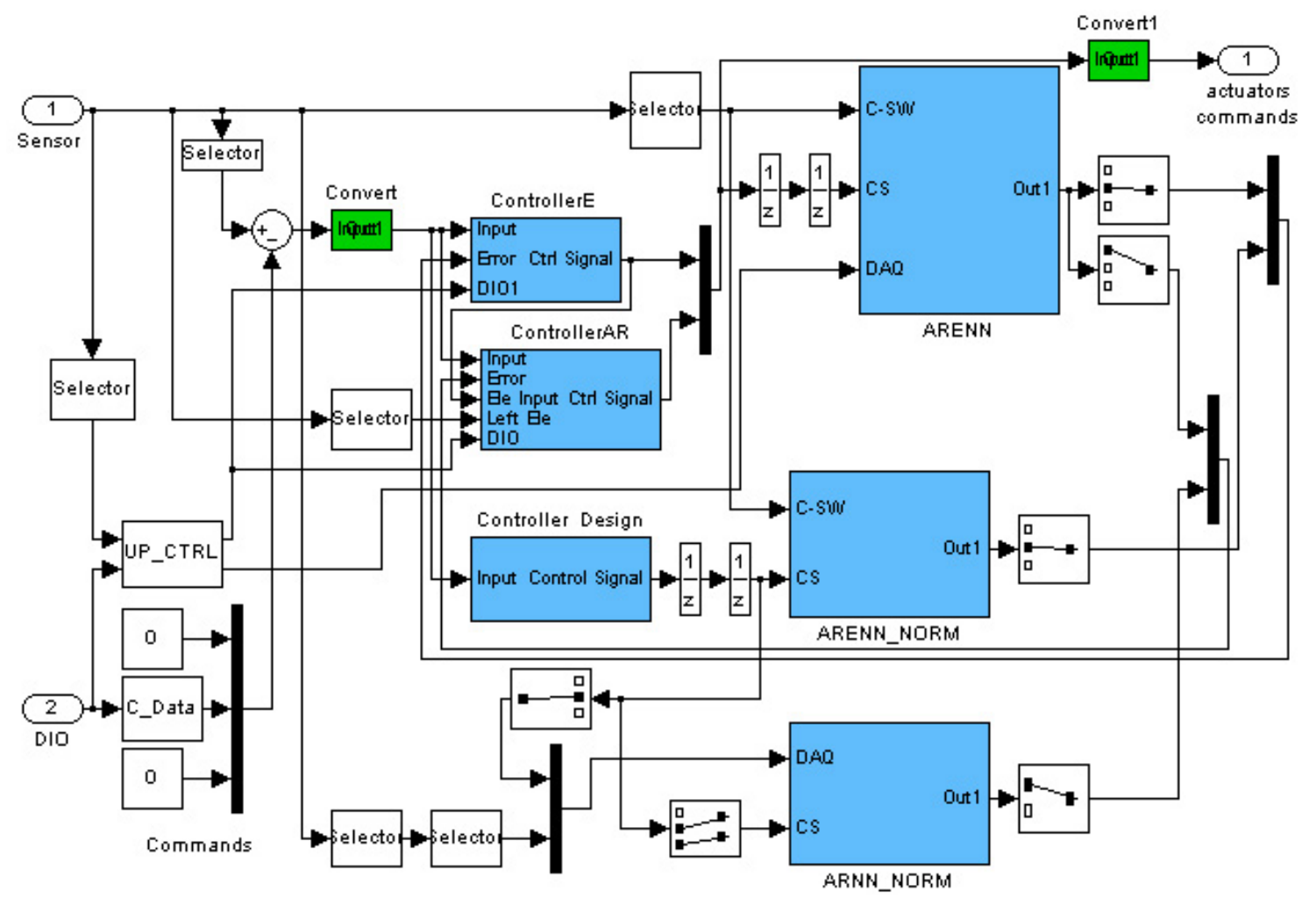

Figure 5-5 Simulink Diagram for Elevator AFA Controller

The weights and thresholds of the pre-trained NNs were stored in a data file. The AFA controller loads this file at the initialization stage of the execution and stores the information into memory. The on-line learning of the NNs is then turned on 
only after the occurrence of the failure. According to the planning of the procedure, once the pilot turned on the controller switch, the failure (aileron/elevator) occurs instantly. The pilot still has control on other channels for an additional four seconds. The NNs will start training instantly. The foursecond-time delay was designed to avoid the initial high approximation error of the NN learning. After four seconds, the on-board controller takes over all of the controls and begins updating the feedback gains in the linear controller to accommodate for the failure.

\section{On-board Servo Calibration}

The control command generated by the on-board AFA controller was in degrees, which were the desired deflection angle of each control surface, while the servo control module requires 8-bit digital signals (between 0-255) as inputs. Thus, the control commands need to be calibrated into a digital signal before feed into the servo control module. The on-board servo calibration software loads the calibration information (acquired with a ground servo calibration) at the initialization stage of the execution and stores them in the memory. During the flight, it converts the control commands and sends the signals to the servo control software.

\section{Servo Control}

The servo control block is the final stage of the flight control software. It sends the calibrated control commands to the serial port. The servo control module on the controller board will then convert this signal into multi-channel Pulse Width Modulation (PWM) signal. Once the control switch is activated, these signals will be used for controlling the aircraft. The following six channels are controlled independently on the aircraft:

- Left Elevator;

- Right Elevator;

- Left Aileron;

- Right Aileron; 
- Left/Right Rudders (one signal);

- Throttle.

The on-board controller can have full control of the aircraft and have the capability of injecting the elevator and/or aileron failure.

\section{4 - Calibration Software}

In addition to the real-time flight control software, a set of supporting software was also necessary for flight testing. One of the major categories is the aircraft ground calibration software, which was essential for the accuracy and safety of the flight control system. These include surface calibration software, servo calibration software and trim position detection software. Each of these specific software schemes will now be outlined.

\subsubsection{Surface Calibration}

The surface calibration software measures the relationship between the aircraft's major control surfaces and the potentiometers linked to them. In addition, the two potentiometers in the nose probe were also calibrated with this software. This information was then used by the DAQ software to measure the actual deflection of each control surface and flow angles via potentiometers.

In a surface calibration procedure, the operator moves each surface to the maxim positive deflection, zero deflection and the maximum negative deflection sequentially. The calibration software then measured the voltage output of each potentiometer with the DAQ card. These voltages were saved and used to calculate the gain/offset information with a linear fitting method.

\subsubsection{Servo Calibration}

Servo calibration software provides the information for the on-board controller to convert control commands into actual surface deflections. The servo calibration procedure was designed to be performed after the surface calibration and it is fully automated. The software sends out servo control signal to each servo and scans the whole range of the control surface travel. At the same time, the calibration software 
measures the deflection of each control surfaces and stores the servo command accordingly. The calibration results are then stored on the compact flash card to be used by the control software.

\subsubsection{Trim Position Detection}

With the different configuration of the flight test, the trim positions for the different aircraft control surfaces may change by small values. The trim position detection software was designed to find out the trim position of each control surface and this information was provided to the on-board controller. During a trim position detection procedure, the operator turns on the $\mathrm{R} / \mathrm{C}$ system and keeps all the control surface at the trim position of the previous flight. The calibration software will then read these positions and store them in memory. Next, the servo control command was sent to each servo to move the control surfaces through the entire deflection range. The position that matches the trim position was detected and the servo command at that moment was stored in the file. 


\section{Chapter 6 Flight Testing}

Flight testing was the most critical phase of this research work. The flight testing facility is located at the WVU Jackson's mill field about 60 miles south of Morgantown, WV. This facility was secluded from commercial and general aviation air traffic activity; thus, perfectly suited for research flight testing activities. This facility features a 3,200 feet long, 50 feet wide semi paved runway. In this chapter, details about flight testing activities and final test results will be discussed.

\section{1 - Flight Testing Phases}

To meet the requirement of the controller design and guarantee aircraft safety, the flight testing activities for this project were divided into seven phases with different onboard hardware/software configurations and task requirements. Each of the individual phases will now be described in detail.

\section{Phase \#1 Flight for Assessment of Handling Qualities}

The aircraft was flight tested in $\mathrm{R} / \mathrm{C}$ mode only without any electronic payload. The objective was to evaluate the dynamic characteristics of the YF-22 research $\mathrm{UAV}$, as well as determining the trim characteristics and the propulsion system performance.

After the initial few flights, "dummy" payload weights were progressively added to the aircraft model; building up to a final weight configuration of an aircraft representing a full electronic payload. This allowed for an evaluation of the aircraft payload capability and handling qualities/performance at the standard operational flight test configuration. This phase of flight testing was completed within six flights and the pilot reported desirable aircraft handling qualities.

\section{Phase \#2 Data Acquisition Flights}

After detailed evaluation of the aircraft dynamic characteristics, on-board instrumentation and computer equipment were then installed. The on-board computer collected flight data from all aircraft sensors and stored the information on a compact flash card for post-flight analysis. The purpose was to acquire flight 
data for parameter identification purposes and testing the following aircraft subsystems:

- Sensor systems;

- $\mathrm{OBC}$;

- Data acquisition hardware/software;

- On-board power system;

- Aircraft EMI

For parameter identification purposes, to obtain an aircraft mathematic model, a set of dedicated PID maneuvers were performed:

- Elevator doublets;

- Aileron doublets;

- Rudder doublets;

- Aileron-rudder doublets combination.

The linearized mathematic model of the aircraft used for the controller design, was then estimated from this set of flight data. In addition, the flight data was also used for training the AFA NNs.

Sample data of aileron-rudder double combination and elevator doublet are shown in Figures 6-1 and 6-2. Sample data is from a flight-test on July $17^{\text {th }}$ 2003, specifically flight number \#3. Figure 6-1 shows a typical aileron-rudder doublet combo used to estimate the aircraft mathematic model for lateraldirectional dynamics. Figure 6-2 shows typical elevator doublet used to estimate the longitudinal mathematic model of the YF-22 aircraft. This phase of flight testing was completed within three flights. 

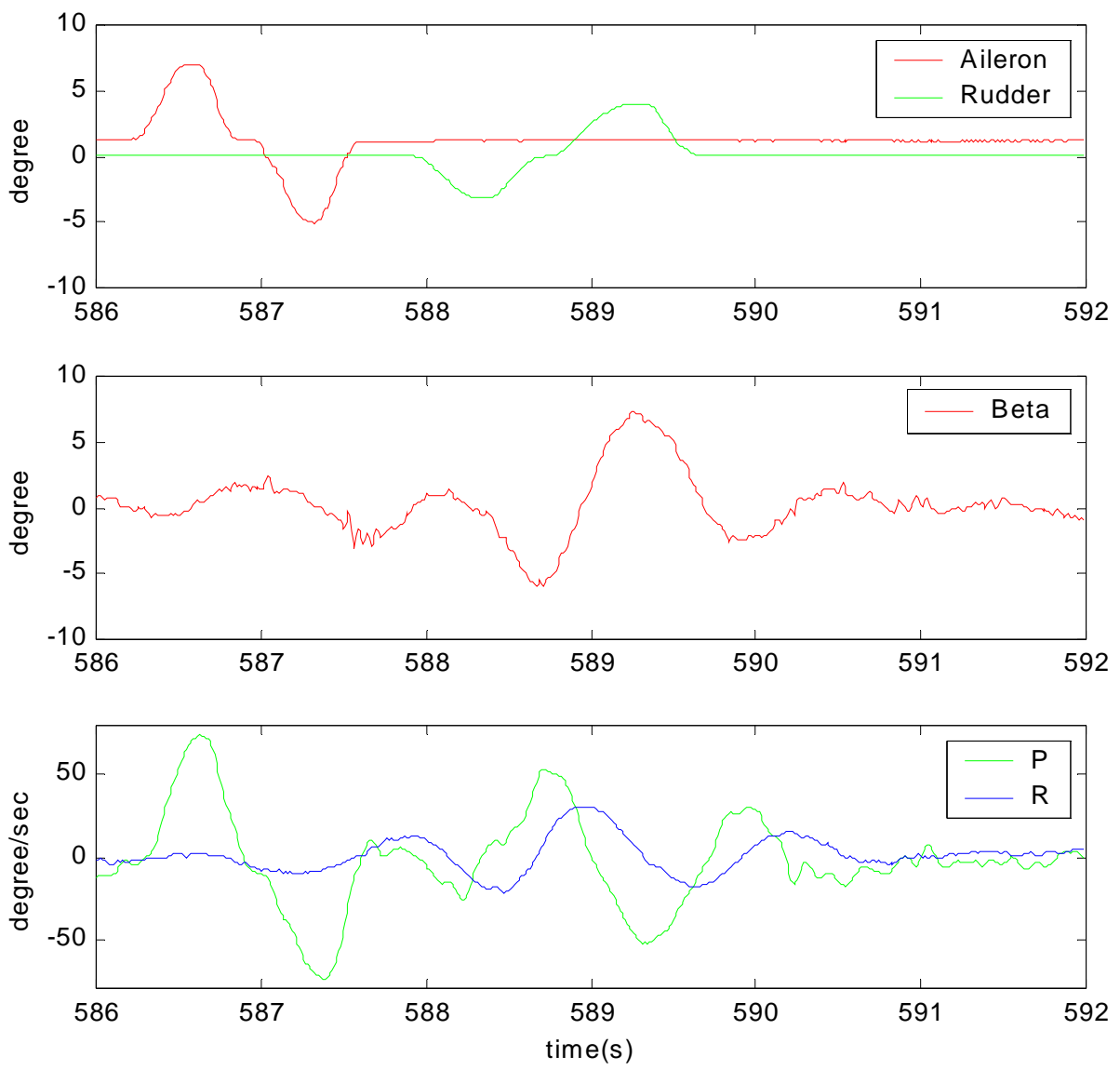

Figure 6-1 Flight Data Following Aileron-Rudder Doublet combination 

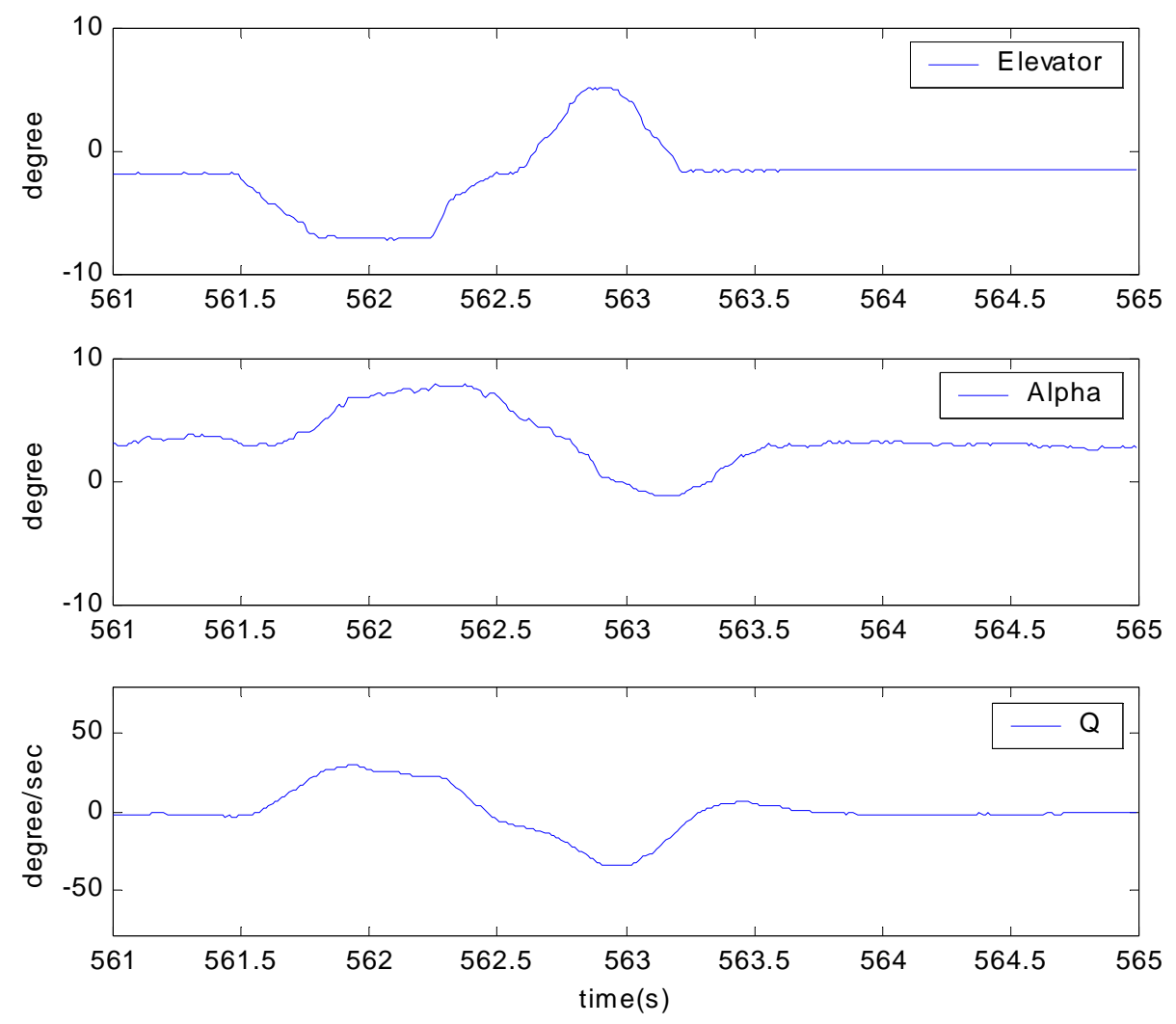

Figure 6-2 Flight Data Following Elevator Doublet

\section{Phase \#3 Data Acquisition Flights (with failure)}

In this phase, additional on-board hardware/software for failure triggering were installed. To guarantee the safety of the aircraft, the "failure triggering" mechanism in the YF-22 on-board payload system was designed to be extremely reliable. Two types of actuator failure were injected: aileron failure and elevator failure. In both modes, the right side of the actuators was locked at the trim position while the rest of the control surfaces were manually controlled by the UAV pilot. The aircraft was under manual control for takeoff and landing. The commands for injecting and removing the failure mode were sent via the pilot with the controller switch during a flight mission. No automatic controller was involved during this stage of flight testing. Giving the criticality of the task, the pilot had to pay extra attention and release the failure situation once the aircraft fell into any unsafe conditions. A set of dedicated PID maneuvers was performed 
with the remaining control surfaces. The flight data files were then used for the failure analysis, in both AFA NN training and AFA controller simulation. The failure trigger hardware/software was also fully tested during this stage and proved to be reliable.

Sample data of right aileron failure is shown in Figure 6-3. Once the controller switch was active, the right aileron was locked at the trim position, and the pilot had control of the left aileron. Sample data is from Oct $5^{\text {th }} 2003$ flighttest. This phase of flight testing was completed within two flights.
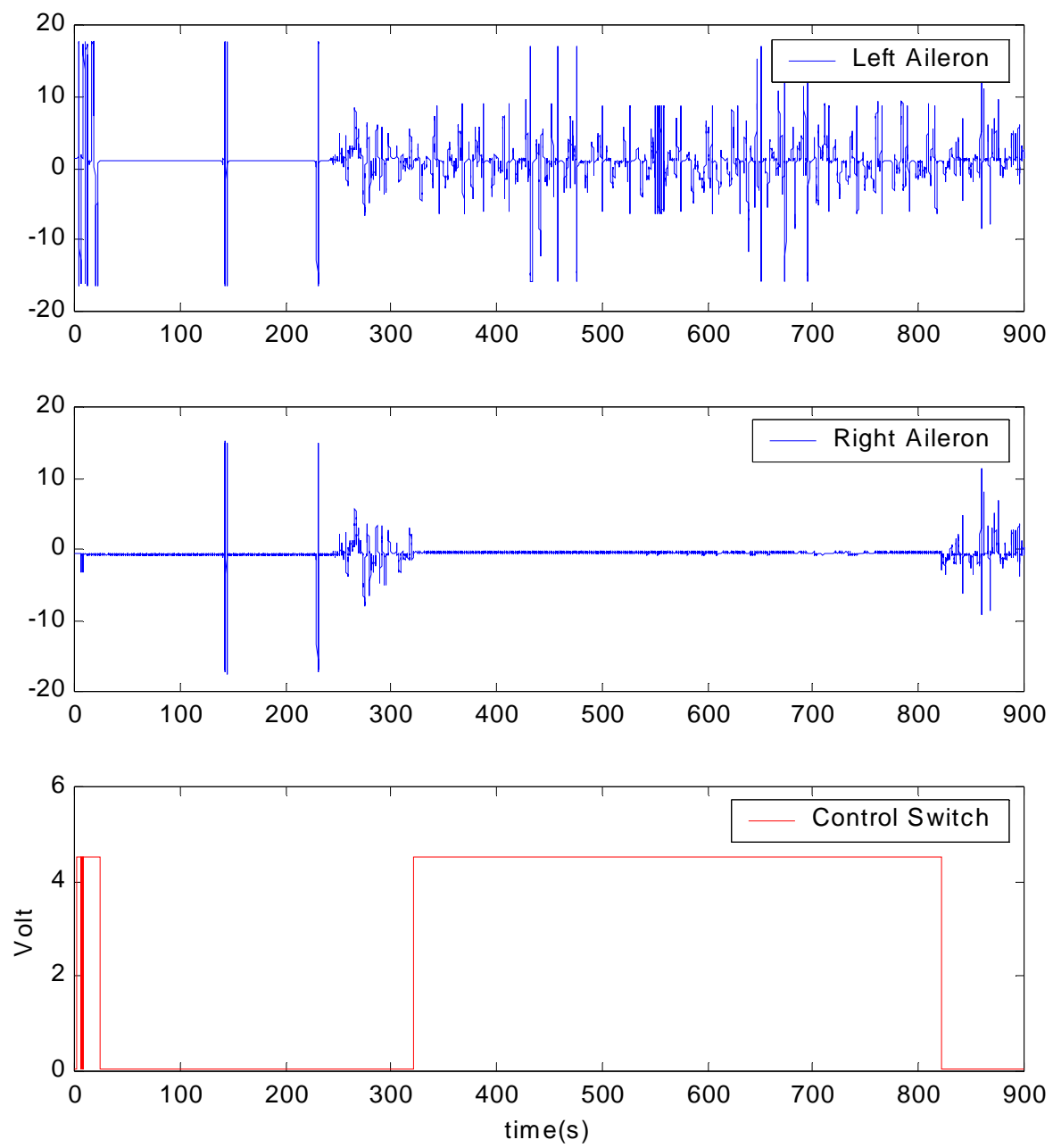

Figure 6-3 Aileron Deflection with Failure 


\section{Phase \#4 Linear Controller Flights (without failure)}

With the mathematic model acquired from Phase \#2, a linear controller was designed to stabilize the aircraft at nominal flight conditions. The designed linear controller was installed in the on-board computer. This was the first set of testing where the pilot had no major control of the aircraft except for the engine throttle. The pilot could regain the control of the plane at any moment during the flight by disengaging the controller switch. The goal for this phase was to evaluate the accuracy of the estimated mathematical model and validate the linear controller design. This provided a basis for the rest of the AFA controller design.

Sample data collected from linear controller validation flights are shown in Figure 3-24 though 3-26. The designed linear controller showed a satisfactory performance to stabilize the aircraft at nominal flight condition without actuator failure (Section 3.4.3). This phase of flight testing was completed within four flights and the linear controller designed exhibited desirable performance.

\section{Phase \#5 Linear Controller Flights (with failure)}

In this phase, the same linear controller was installed on the on-board computer along with the failure triggering software. Both types of failures were tested with the linear controller activated. The flight data was stored in the compact flash card and were used to compare with final flight test data with the AFA controller.

Sample data collected from this phase of flight testing were shown in Figure 3-29 though 3-30 for aileron failure and Figures 3-37 though 3-39 for elevator failure. From these plots, it can be concluded that the linear controller does not have the ability to adapt to the failure condition and cannot maintain the handling quality of the aircraft after the actuator failure (Section 3.5). Flight testing activity for this phase was completed within four flights.

\section{Phase \#6 Aileron Failure with AFA Controller}

This phase started after all the AFA software was simulated and fully evaluated. The aircraft took off manually with the pilot control and the on-board 
controller off. Once the plane reached a safe altitude the pilot could activate the controller switch. The right aileron would then enter into the failure mode (locking at the trim position) instantly while the pilot retained control of all other control surfaces for four seconds. The on-line learning NNs would begin training at the same time. During this period, the pilot was asked to perform aileron maneuvers to help the NN learn about the lateral dynamics after failure. After four seconds, the controller would take over all the rest control channels (except for engine throttle) and begin updating the controller gain to accommodate for the failure. This process was repeated several times during a flight test with the pilot deciding to conclude the test and regain control instantly by using the controller switch.

This phase of flight testing was completed within seven flights and the flight data collected will be presented in section 6.3.1.

\section{Phase \#7 Elevator Failure with AFA Controller}

This phase shares basically the same procedures as Phase \#6 but for the case of elevator failure. Once the pilot activated the controller switch, the right elevator would enter the failure mode (locked at a trim position) instantly and the on-board NNs would start training at the same time while the pilot retained control of the rest surfaces for four seconds. During this period the pilot was asked to perform an elevator doublet to help the $\mathrm{NN}$ with longitudinal dynamics learning. After four seconds, the controller would take over the remaining control surfaces and updating the feedback controller gain. The updated gain would increase the controller's ability to control the aircraft after failure and compensate for the rolling moment caused by a single elevator failure.

This phase of flight testing was completed within four flights and the flight data collected will be presented in Section 6.3.2. Details about the flight testing procedure will be discussed in the next section. 


\section{2 - Flight Testing Procedures}

The flight testing procedures typically included pre-flight preparations, in-flight procedures, and post-flight data analysis. After several years of flight testing activities at WVU, the flight testing procedure have been refined and optimized to a very high degree of reliability and efficiency.

\subsubsection{Pre-flight Preparation}

The "Pre-Flight Preparation" includes the following set of procedures:

- Aircraft mechanics are reviewed, including control surfaces, battery systems, landing gear, brakes, etc;

- Control surface calibration for each aircraft control surface to provide an accurate reading;

- Servo calibration for each servo to have an accurate control;

- DAQ system validation;

- Controller validation. The failure trigger mechanism and controller switch are tested prior to the flight operations;

- System start-up. Once the aircraft is on the runway, the vertical gyro is leveled before powering up the payload. After approximately three minutes (warm up time for the gyro), payload system is cleared for flight;

- $\mathrm{R} / \mathrm{C}$ ground range check. To guarantee aircraft safety, ground testing of the $\mathrm{R} / \mathrm{C}$ radio system with all the on-board payload system active (along with the propulsion systems) is found acceptable ( $>300 \mathrm{ft}$ ground range with transmitter antenna fully retracted) for flight operations.

\subsubsection{In-flight Procedures}

In-flight procedures varied from task to task during the flight sessions depending on the phase. The procedure for the final actuator failure accommodation tests will be discussed in this section.

In-flight procedures were designed to provide a safe and efficient way to demonstrate actuator failure accommodation control with the UAV test bed. Several major difficulties were encountered during the flight tests: 
- Restricted airfield: flight tests were limited within the visual range since the aircraft was under manual control for takeoff and landing purposes. The aircraft's on-board controller does not have the ability to automatically circle the airspace. Overall, the pilot had to engage and disengage each time the airplane reached a leg of the airspace. This limited the time available for each AFA controller engagement to be short, typically between 12-16 seconds, which are not long enough to finish a full-blown AFA test.

- Neural network training: the NN could not learn the aircraft's dynamics efficiently under straight and level flight conditions. This issue is well known in the area of parameter identification. Aircraft control surface inputs were necessary to "excite" the vehicle dynamics.

- Wind gusts: since the UAV has only a $6.5 \mathrm{ft}$ wing span, wind gust posed a major problem for on-line NN training. On average 5-10 mph winds are always present during a flight, leading to $20-40 \mathrm{deg} / \mathrm{sec}$ disturbances for roll rate measurements. This strong disturbance caused a very low signal/noise ratio and created a negative effect on updating the feedback gains.

To solve or minimize the effect of these problems, special care was taken during the design of the flight procedure. Several flight procedures were tested and the final version used for AFA test is shown in Figure 6-4. 


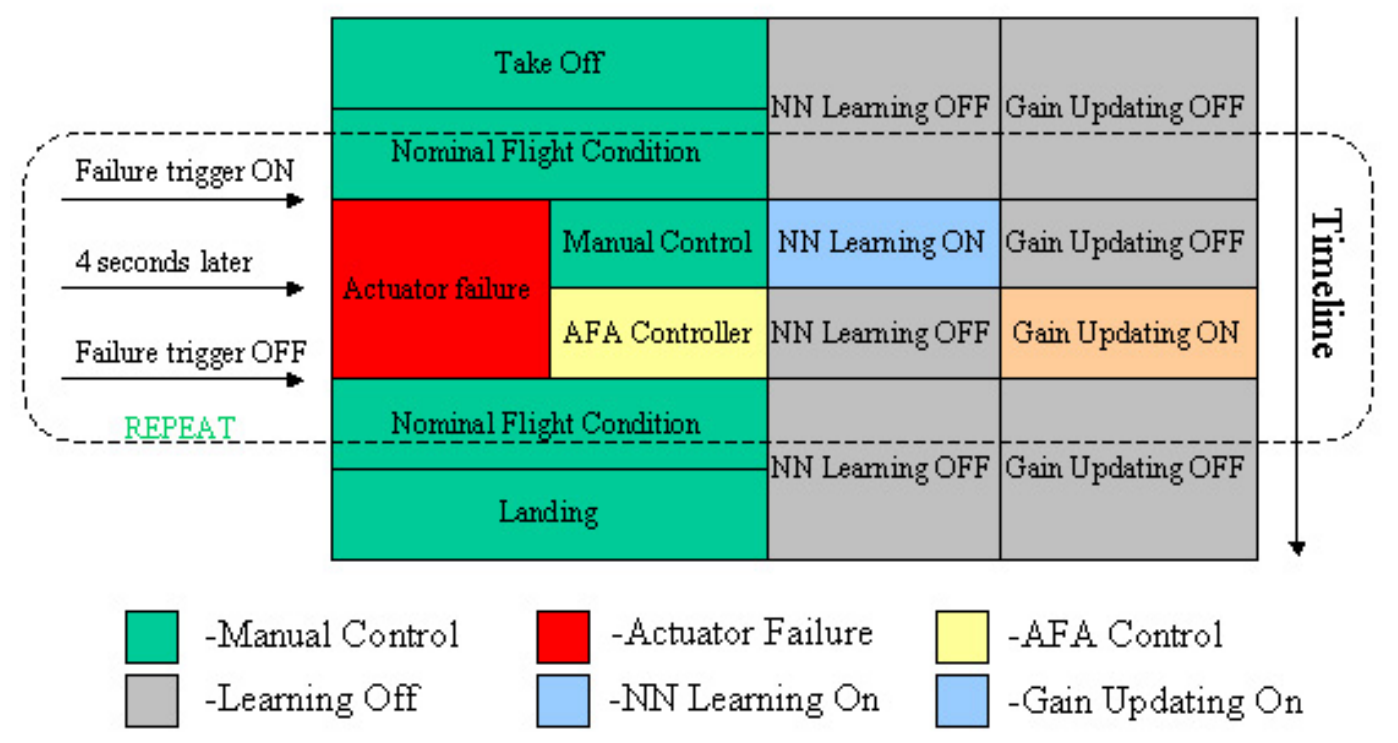

Figure 6-4 In-flight Procedures

Within this procedure, the aircraft takes off manually with the ground control. After reaching the desired flight condition (about $150 \mathrm{~m}$ high and $40 \mathrm{~m} / \mathrm{s}$ ), the pilot begins to activate the controller switch. The actuator failure triggers instantly with the on-line learning $\mathrm{NN}$ training from real-time flight data. The pilot remains in control of all other control surfaces for four seconds. During this period, the pilot can then perform certain maneuvers (i.e. aileron doublets, elevator doublets, etc) to excite the aircraft dynamics. The controller feedback gain will not be updated during the maneuver.

After four seconds, the AFA controller will take over aircraft control. The NN training will then be deactivated. The feedback controller gain will be updated with the estimation from both the on-line and off-line learning NNs. The reason the NN learning is not active at the same time as feedback controller gain updating is due to the disturbance of wind gusts. With the existence of high disturbance, the estimation of the NN would then be affected during the training process. This effect is much less noticeable while the $\mathrm{NN}$ has not been training at the same time; this is because the $\mathrm{NN}$ acts as an integrator. A sine-wave pattern command was feed into the controller at this stage of the flight testing to simulate the human pilot input. This would help exciting the dynamics of the aircraft for feedback gain updating and provide a way to validate the controller performance during the post-flight data analysis. 
Once the aircraft reaches the end of the flight field, the pilot can turn the controller switch off, turn the aircraft manually and reset for the next engagement. This process is then repeated several times throughout the flight test mission. The on-line learning NN and linear feedback controller gain are then updated from the previous values. In this way, the actuator failure accommodation test can be divided into several relatively small time-slots but still can have enough total time to complete the task at hand. A drawing of a typical flight pattern is shown in Figure 6-5.

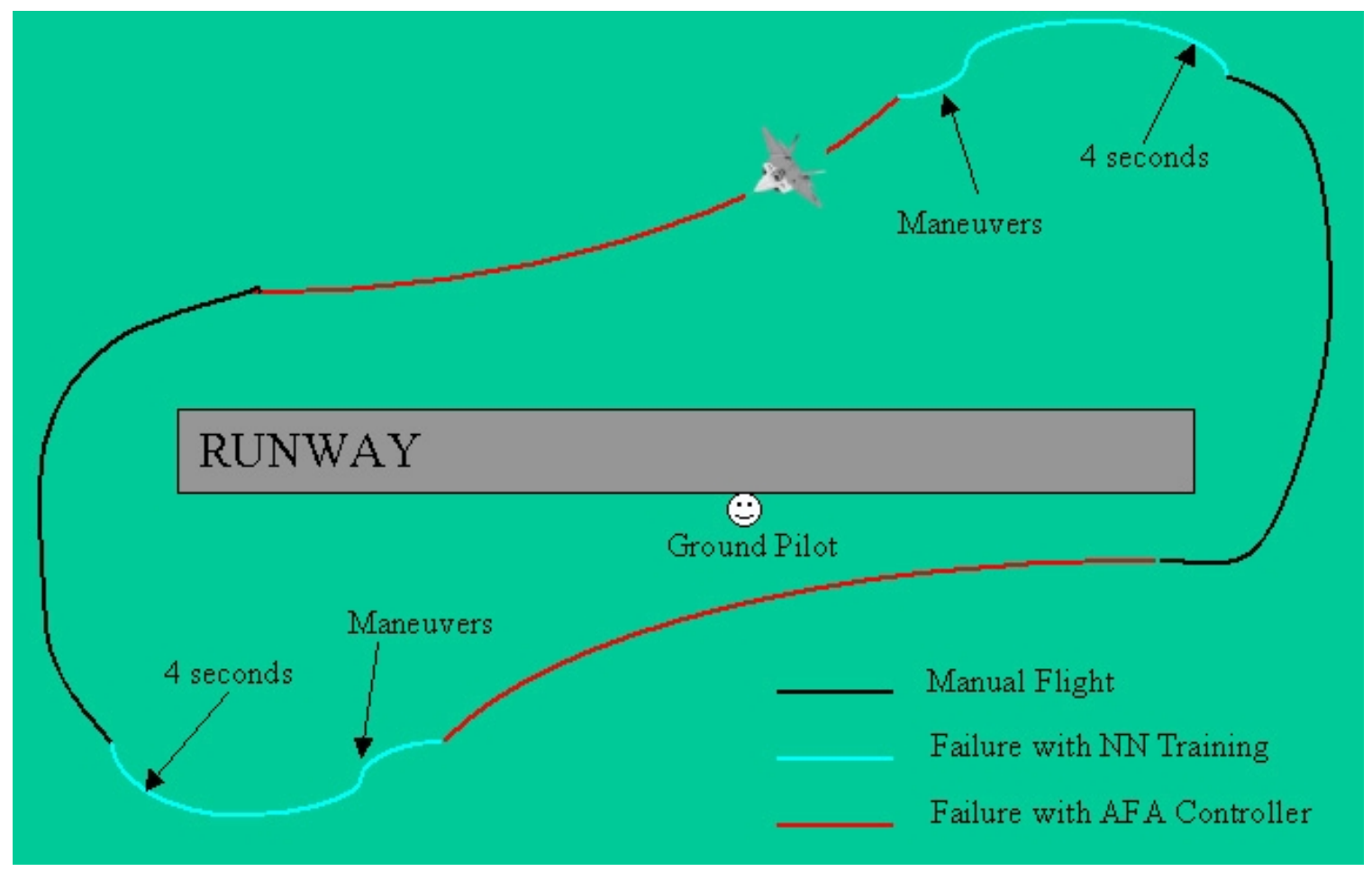

Figure 6-5 Flight Path

During the flight test, if anything abnormal happens to the aircraft, the pilot switches back to manual control using the controller switch on the transmitter. Once the mission tasks have been completed or a pre-defined flight time was reached, the pilot begins the landing procedures. During this time, the controller switch will be turned off and the aircraft landed under manual control. This procedure was used for the final phase of AFA flight-test with successful results. 


\subsubsection{Post-flight Analysis}

Post-flight analysis typically consisted of flight data downloaded via the compact flash card, converting flight data to meaningful engineering values, and comparing with simulation results. A detailed data analysis for the final AFA flight testing will be presented in Section 6.3.

\section{3 - Final Test Results}

With all early phases of the flight testing been completed and all the AFA controller been fully simulated, flight testing progressed towards final actuator failure accommodation tests in 2004 flight season. A selection of flight data collected from both aileron failure and elevator failure AFA tests will be presented in section 6.3.1 and 6.3.2.

\subsubsection{Aileron Failure AFA Test}

Figures 6-6 though 6-15 show the flight data collected from the aileron failure AFA test on Oct. $06^{\text {th }}, 2004$ flight testing session. The ground temperature was approximately $70{ }^{\circ} \mathrm{F}$ with less than $5 \mathrm{mph}$ wind. The aircraft was launched fully fueled at around 4:30pm. The total flight duration was 600 seconds; the controller switch was activated 19 times during the flight. The total duration of controller switch activation (w/aileron failure) was 228.28 seconds. The total time of on-line $\mathrm{NN}$ training was 76 seconds and total time for controller gain updating was 133.28 seconds. After each activation of the AFA controller, the aircraft software was designed to track a sine-wave roll rate pattern. A full view of the aileron commands and controller switch activation is shown in Figure 6-6. Once the controller switch was activated, the right aileron was locked at the trim position. 

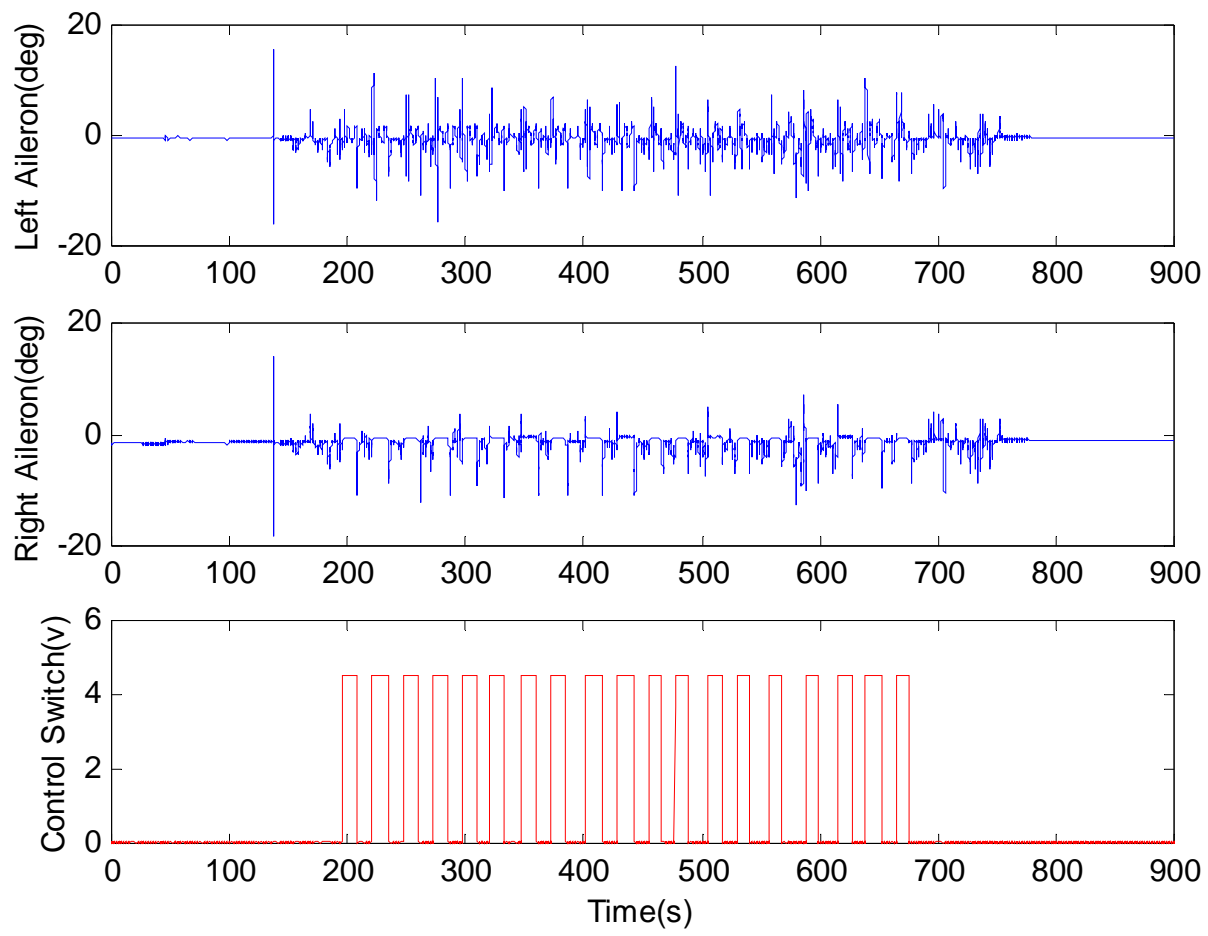

Figure 6-6 Aileron Inputs

Both the on-line and off-line $\mathrm{NN}$ were pre-trained using previous flight data without a failure. The goal of the training was to approximate the lateral-directional dynamics of the aircraft under typical flight conditions. During the flight testing, the online $\mathrm{NN}$ was trained to approximate the lateral-directional aircraft dynamics after failure. The difference between the outputs of both NNs was used by the AFA controller to tune the roll rate feedback controller gain. The learning rate for the on-line learning $\mathrm{NN}$ was set at 0.2 and the learning rate for the roll rate feedback gain updating was set at 5.0e-5. The roll rate feedback gain, starting from 0.04 , and gradually increased until the value stabilized at 0.0732 shown in Figure 6-7. 

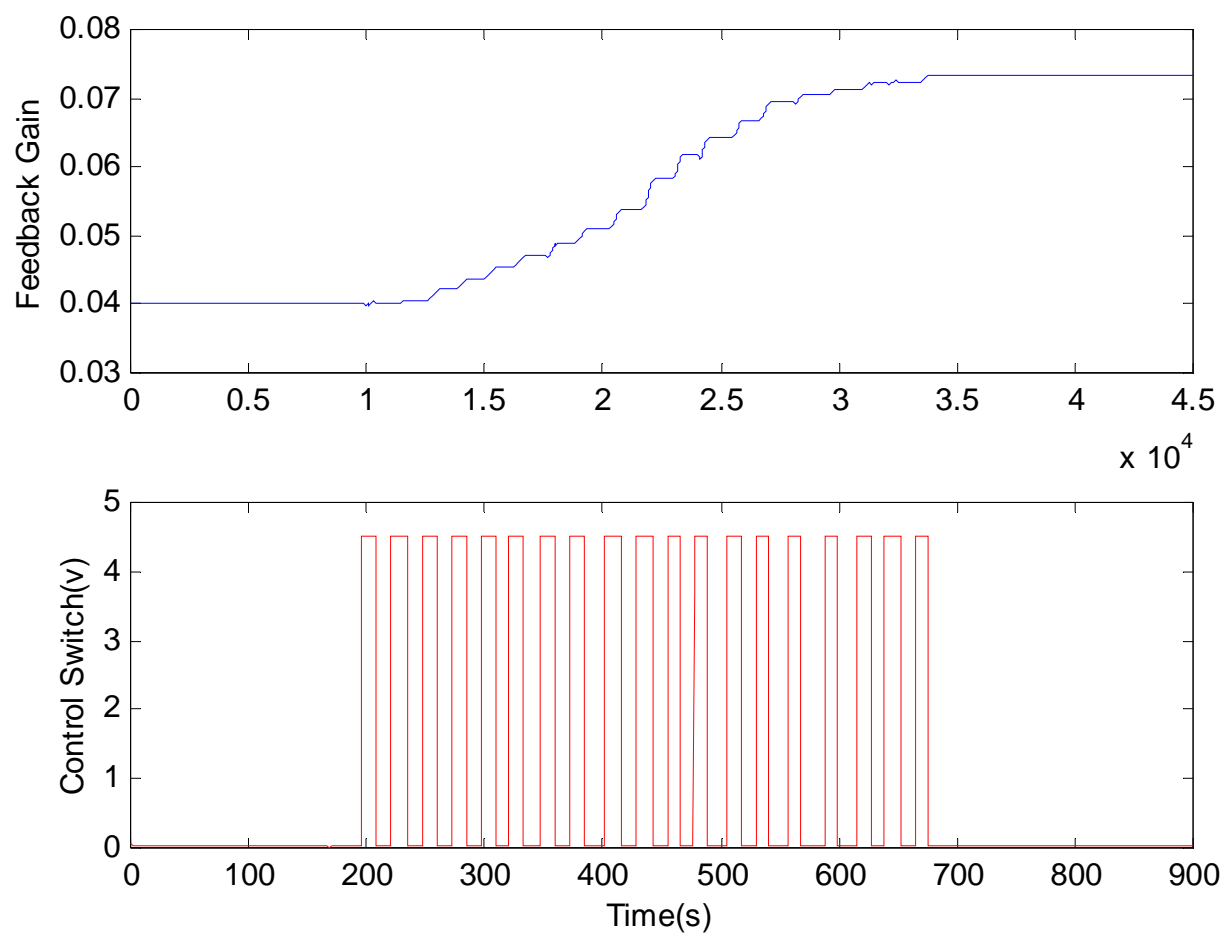

Figure 6-7 Roll Rate Feedback Gain

For a more detailed understanding of the NN learning and gain updating procedure, the flight data was fed into the simulator to playback the actual response of the NN outputs during the flight. Figure 6-8 illustrates the NN estimations and the gain updating process from the $10^{\text {th }}$ activation (429.10-442.42s). Estimates of the on-line learning $\mathrm{NN}$ are shown with the solid line. The dot line refers to the reference estimation from the off-line learning NN. During the first four seconds, after the controller was activated, the on-line learning $\mathrm{NN}$ was trained with the real-time flight data. The estimation of the on-line learning NN was affected by the wind gust at this moment and could not be used to update the controller gain. After four seconds, the on-line learning was turned off and the estimation difference between the on-line and off-line NN was used to update the roll rate feedback gain. The difference between the two estimations was limited but large enough to update the value of the gain. The roll rate feedback gain starting from 0.0539 at the beginning of this activation had been increased to 0.0584 at the end. 

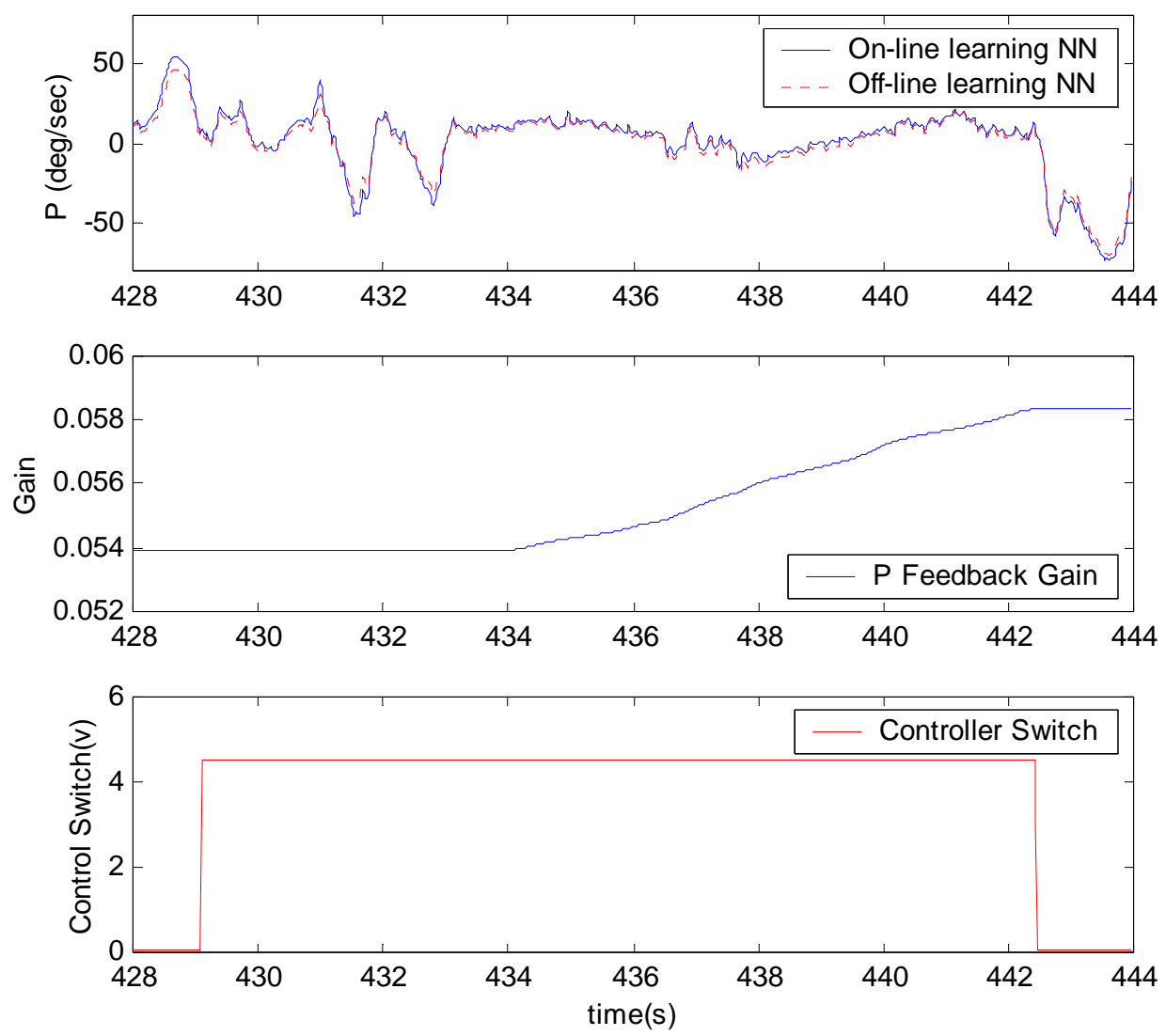

Figure 6-8 NN Approximations

Figure 6-9 shows the left aileron input and the roll rate response for the first activation (194.72- 207.42s) while the AFA controller just started to compensate for the failure. The red circles indicate the four sec mark and where the AFA controller was activated after the mark. Figure 6-10 shows the same signals during the last activation (664.60- 675.14s) - which was $19^{\text {th }}$ for this particular flight - where the AFA controller almost finished the feedback gain updating process and provided a best possible accommodation for the right aileron failure. 

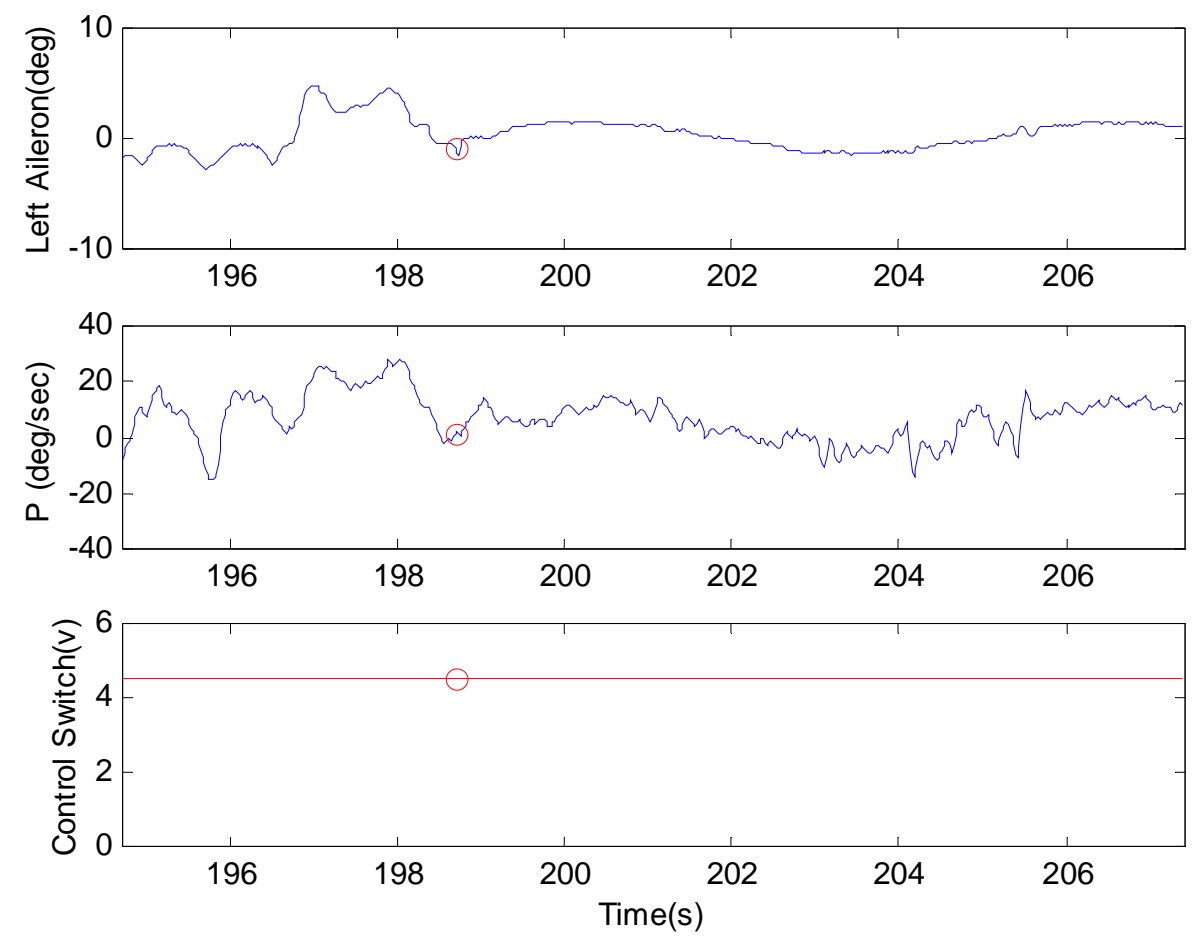

Figure 6-9 Roll Rate Response (First Failure Activation)
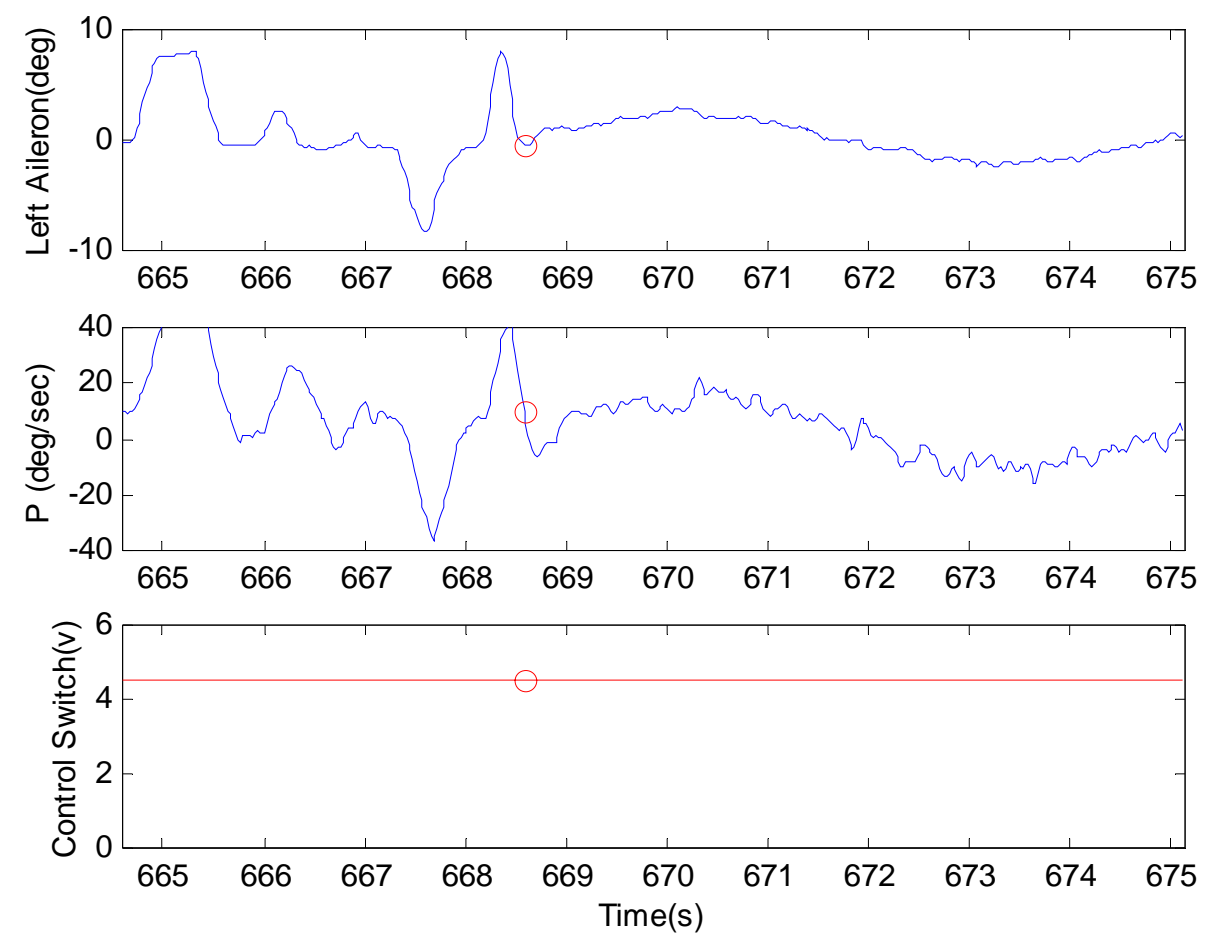

Figure 6-10 Roll Rate Response (Last Failure Activation) 
To facilitate a comparison, the aileron input and the roll rate response of the first and $19^{\text {th }}$ activation had been put side by side in Figures 6-11 and 6-12 (data shown are after the AFA controller was activated)

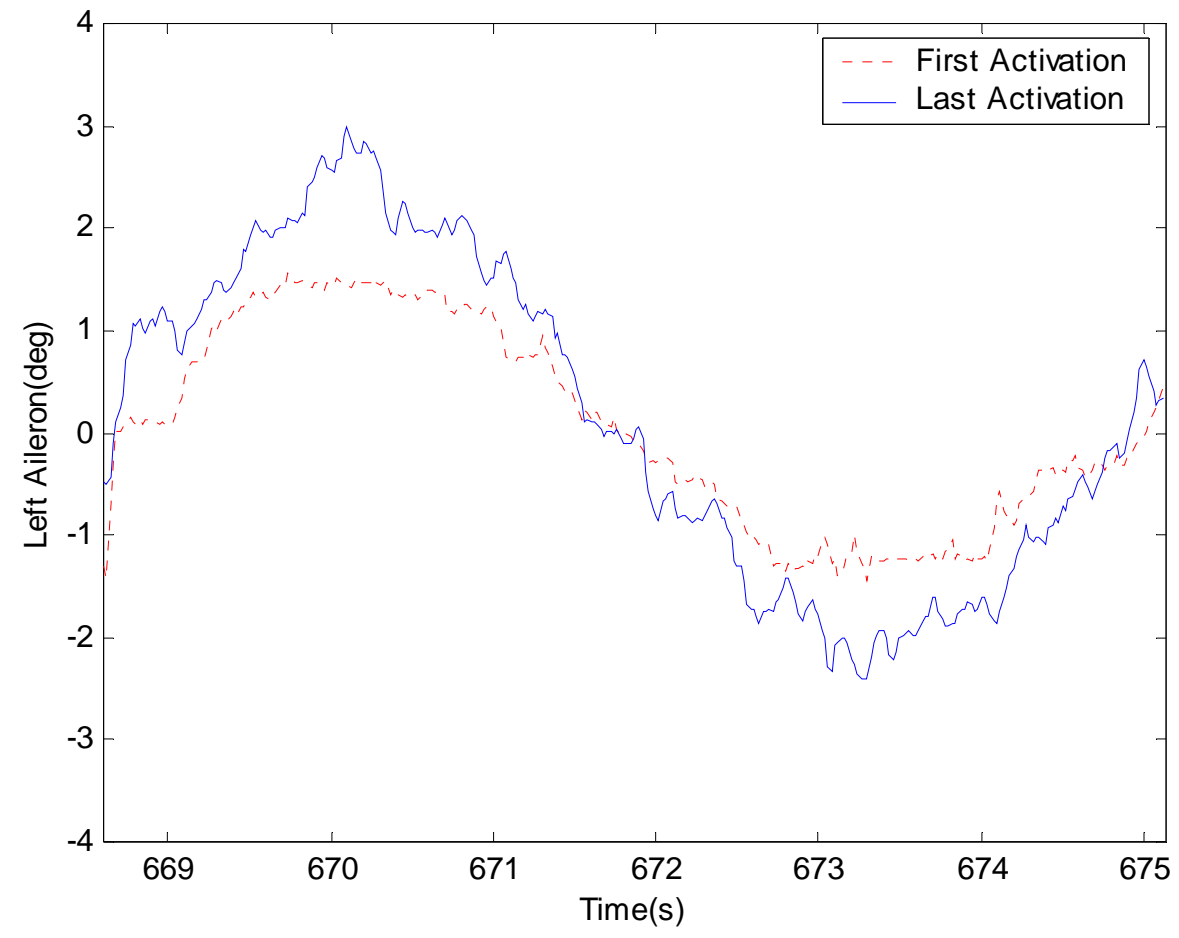

Figure 6-11 Aileron Control Inputs 


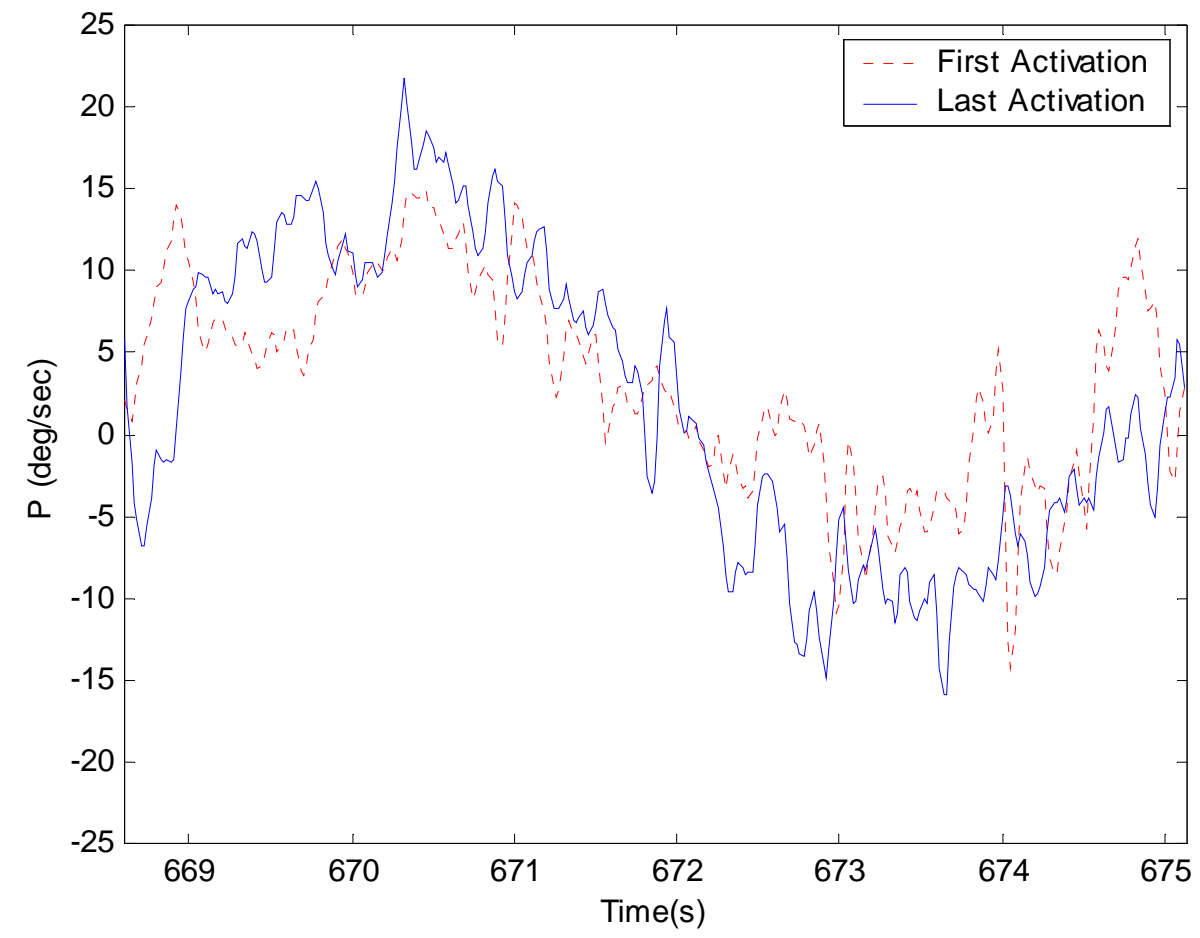

Figure 6-12 Roll Rate Response

It is clearly shown that with the on-line $\mathrm{NN}$ learning, and the updated AFA feedback gain, the control command on the left aileron was increased and the roll rate response shown improvements. To quantify the process of actuator failure accommodation, statistic methods were used to analyze the flight data. The STandard Deviation (STD) of the left aileron deflection and the roll rate response of each controller switch activation are shown in Table 6-1: 


\begin{tabular}{|c|c|c|}
\hline Controller Activation & STD for Left Aileron (deg) & STD for Roll Rate (deg/sec) \\
\hline 1 & 1.0688 & 6.3918 \\
\hline 2 & 1.0719 & 6.2051 \\
\hline 3 & 1.1073 & 6.6104 \\
\hline 4 & 1.1259 & 7.8164 \\
\hline 5 & 1.1736 & 7.6179 \\
\hline 6 & 1.1903 & 8.8733 \\
\hline 7 & 1.1874 & 8.5245 \\
\hline 8 & 1.2241 & 8.9257 \\
\hline 9 & 1.2709 & 9.3998 \\
\hline 10 & 1.4382 & 8.4073 \\
\hline 11 & 1.4628 & 9.3566 \\
\hline 12 & 1.5131 & 9.2610 \\
\hline 13 & 1.6449 & 9.5412 \\
\hline 14 & 1.6120 & 10.7704 \\
\hline 15 & 1.6604 & 9.8754 \\
\hline 16 & 1.8011 & 9.6539 \\
\hline 17 & 1.7129 & 10.4386 \\
\hline 18 & 1.7279 & 11.0050 \\
\hline 19 & 1.7291 & 10.0397 \\
\hline
\end{tabular}

Table 6-1 Statistical Analysis - Aileron Failure

Each STD value was calculated with 4 seconds of flight data after the actuator failure. The starting time of the data selected can be described as:

$$
T_{\text {Start }}=T_{\text {Controller Switch Activation }}+5 \mathrm{sec}=T_{A F A \text { Controller Activation }}+1 \mathrm{sec}
$$

to avoid the initial high correction maneuver after the on-board AFA controller activation. The ending time can be described as:

$$
T_{\text {End }}=T_{\text {Controller Swich Activation }}+9 \mathrm{sec}=T_{\text {AFA Controller Activation }}+5 \mathrm{sec}
$$

to cover most of the flight data after the AFA controller been activated. To facilitate the data analysis procedure, the STD values in Table 6-1 were plotted in Matlab, which is shown in Figure 6-13. 

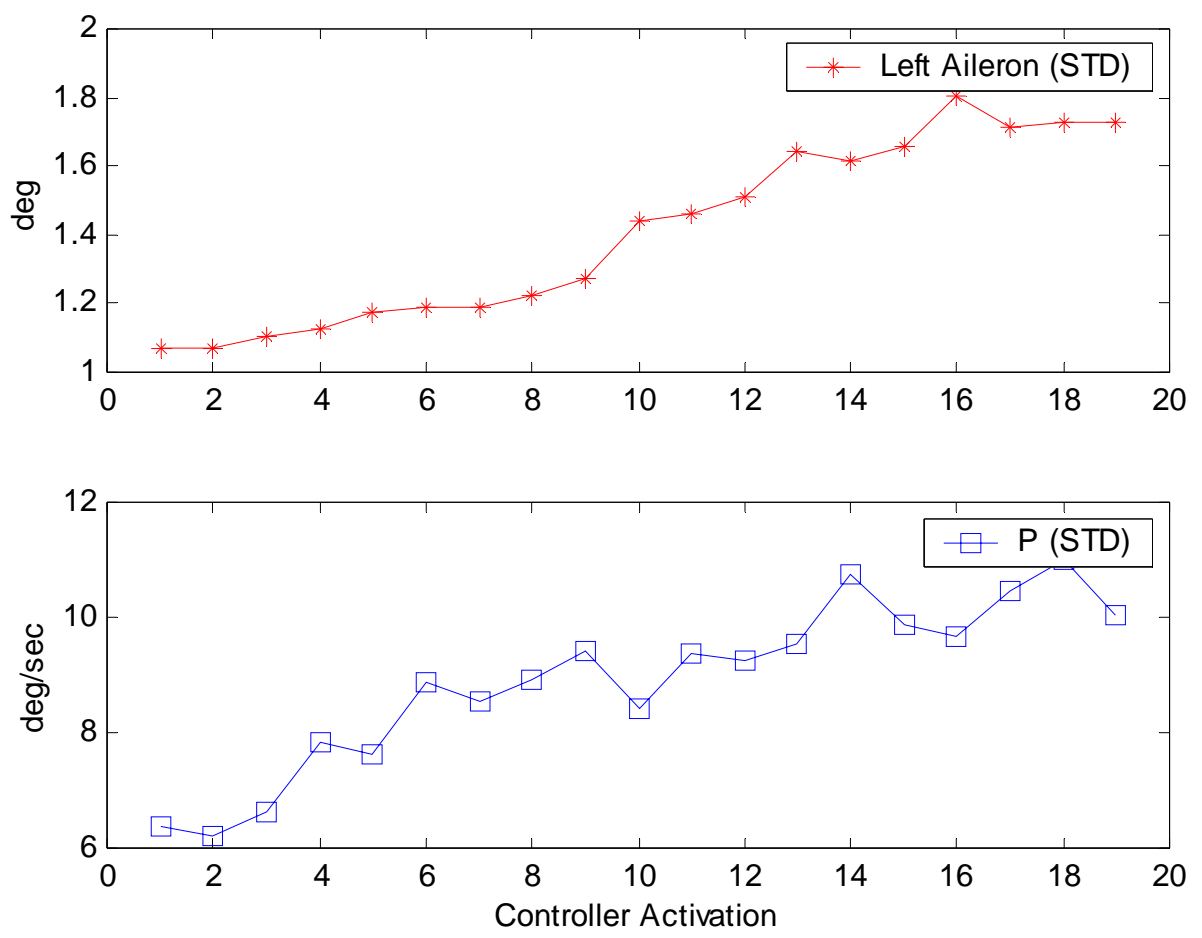

Figure 6-13 Statistical Analysis - Aileron Failure

With the NN on-line learning, the designed AFA controller gradually increased the left aileron control command to compensate for the loss of right aileron. In this way, the designed AFA controller effectively reduced the negative effect caused by the right aileron failure and improved the aircraft's dynamic response.

A performance comparison was performed with three flight conditions:

- Linear controller at nominal flight condition (no aileron failure)

- Linear controller with right aileron failure

- AFA controller with right aileron failure

Flight data collected from flight testing Phase 4 and Phase 5 was used in this comparison. The left aileron deflections for three scenarios are shown in Figure 6-14 and the corresponding roll rate responses are shown in Figure 6-15. 


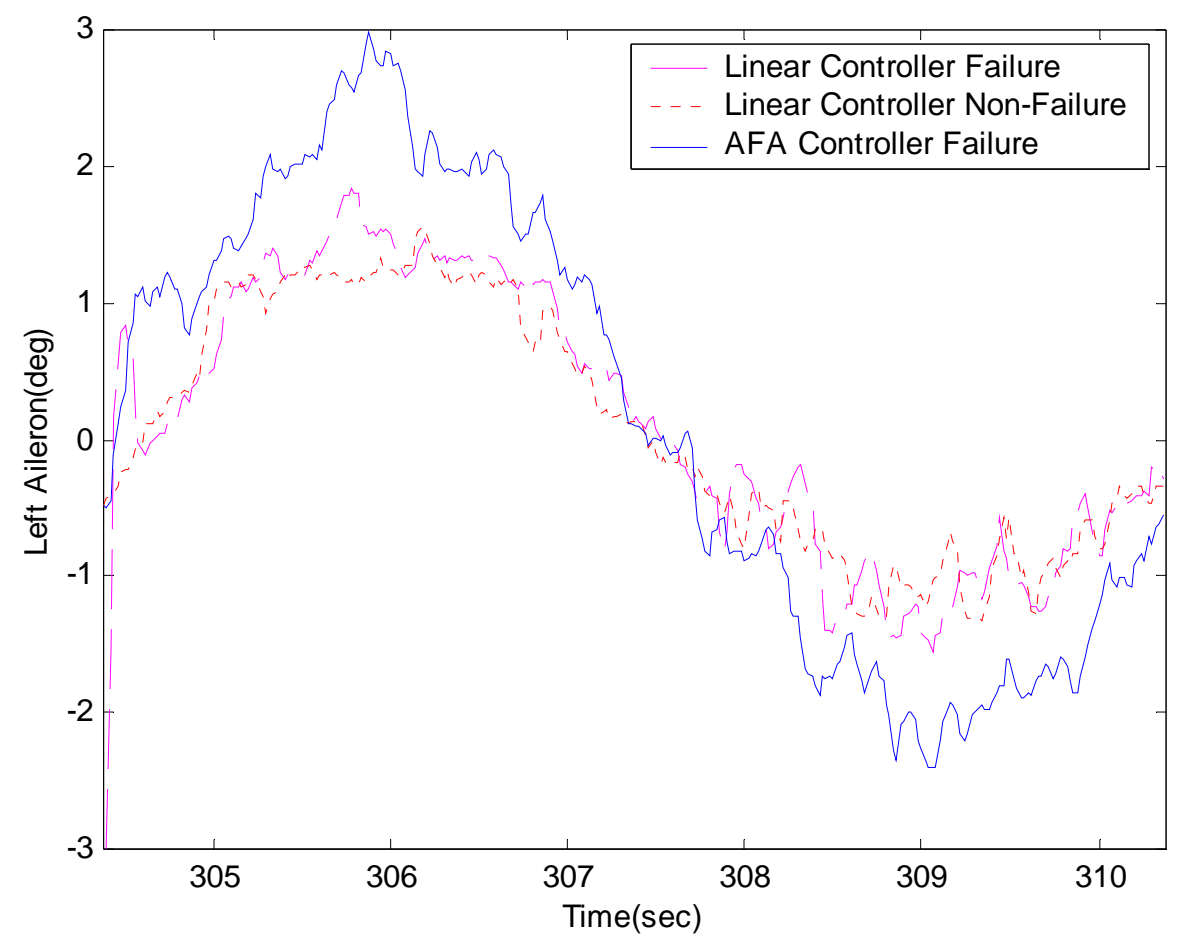

Figure 6-14 Performance Comparison - Left Aileron

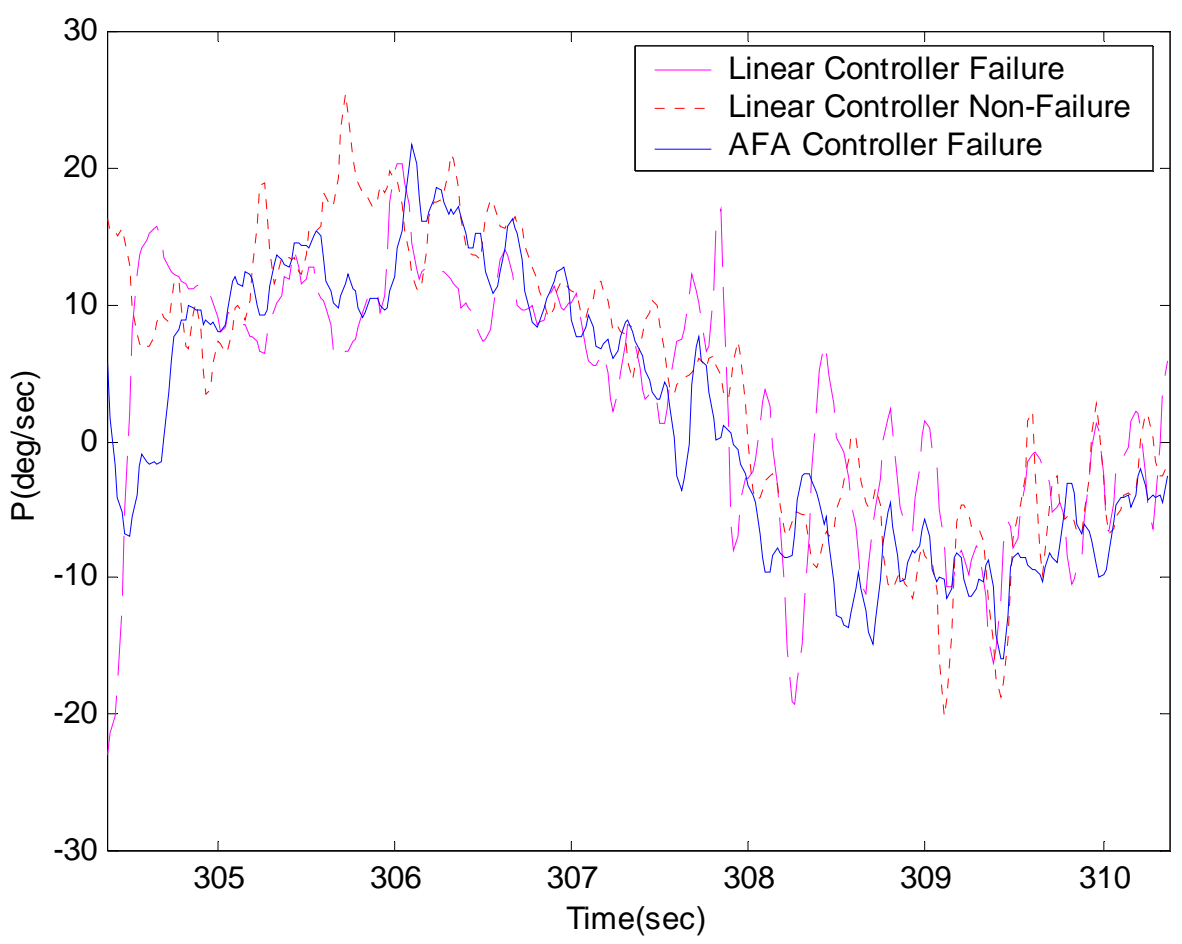

Figure 6-15 Performance Comparison - Roll Rate 
With the AFA controller activated, the left aileron control command had been increased greatly compared with the linear controller command. Due to the existence of wind gust disturbance, the performance difference on the roll rate response was hard to compare from the plot directly. The STD of the roll rate response was calculated and listed in Table 6-2:

\begin{tabular}{|l|c|c|c|}
\hline & $\begin{array}{c}\text { Linear Controller } \\
\text { No Aileron Failure }\end{array}$ & $\begin{array}{c}\text { Linear Controller } \\
\text { Aileron Failure }\end{array}$ & $\begin{array}{c}\text { AFA Controller } \\
\text { Aileron Failure }\end{array}$ \\
\hline STD for Left Aileron (deg) & 0.8910 & 1.0438 & 1.6281 \\
\hline STD for P (deg/sec) & 10.0026 & 8.9359 & 9.6597 \\
\hline
\end{tabular}

Table 6-2 Performance Comparison - Three configurations

With these values, it is clearly shown that the designed AFA controller provided an improved performance over the linear controller under right aileron failure condition and is close to the linear controller performance under the normal flight condition.

\subsubsection{Elevator Failure AFA Test}

Figures 6-16 though 6-31 shows the flight data collected from the elevator failure AFA test on a Sep. $1^{\text {st }} 2004$ flight testing session. The ground temperature was about 80 ${ }^{\circ} \mathrm{F}$ with less than $5 \mathrm{mph}$ wind speed. The aircraft was launched at around 6:30pm with full tanks of fuel. The total flight duration was 523secs; for which the controller switch was activated 20 times during the flight. The total duration of controller switch activation (w/elevator failure) was 226.9 seconds. The total time of on-line NN training was 80 seconds and the total time for updating the controller gain was 126.9 seconds. For each activation of the AFA controller, the aircraft was designed to track a sine-wave pitch rate pattern. A full view of the elevator commands and the controller switch activation is shown in Figure 6-16. Once the controller switch was activated, the right elevator was locked at the trim position. 

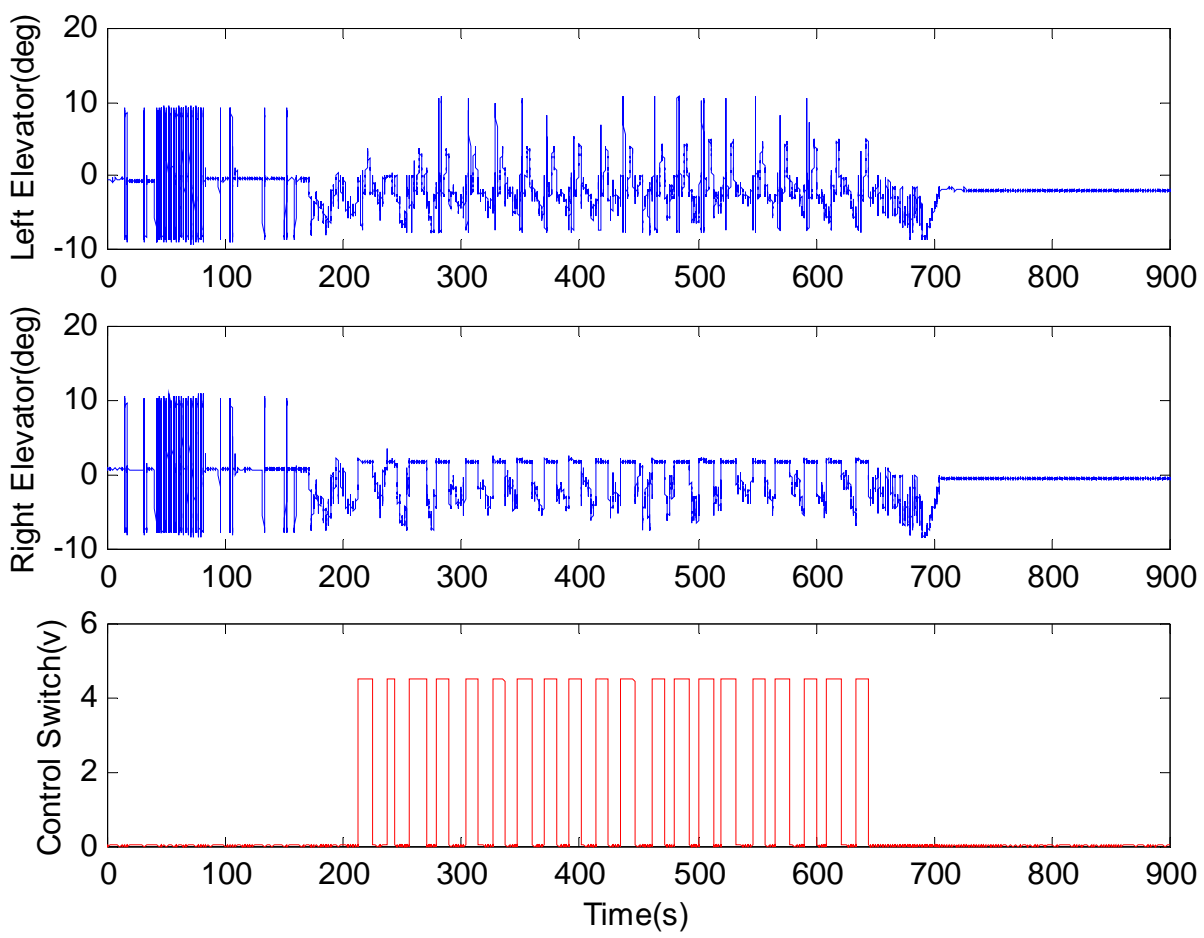

Figure 6-16 Elevator Inputs

Both the on-line and off-line full NN were pre-trained with a previous flight data without failure. The goal was to approximate the dynamics of the aircraft under nominal flight condition. A NN to approximate the lateral-directional dynamics was also used in the controller, which was the same one for the aileron failure AFA test to provide a fully decoupled lateral-directional estimation. During the flight testing, the on-line full NN was trained to approximate the aircraft dynamics after the failure. The estimation difference between the outputs of both full NNs was used by the AFA controller to adjust the pitch rate feedback controller gain. The learning rate for the on-line learning full NN was 0.2 and the learning rate for the pitch rate feedback gain updating was 2.0e-4. The pitch rate feedback gain, starting from 0.12, was gradually increased until stopped at 0.1757. The roll rate estimation difference between the on-line learning full NN and offline learning lateral-directional $\mathrm{NN}$ was used by the controller to compensate for the coupling between the elevator input and lateral dynamics. The learning rate for the decoupling gain updating was 2.0e-4. The decoupling gain, starting from 0, was gradually increased until stabilizing at a value of 0.3711 (Figure 6-17). 

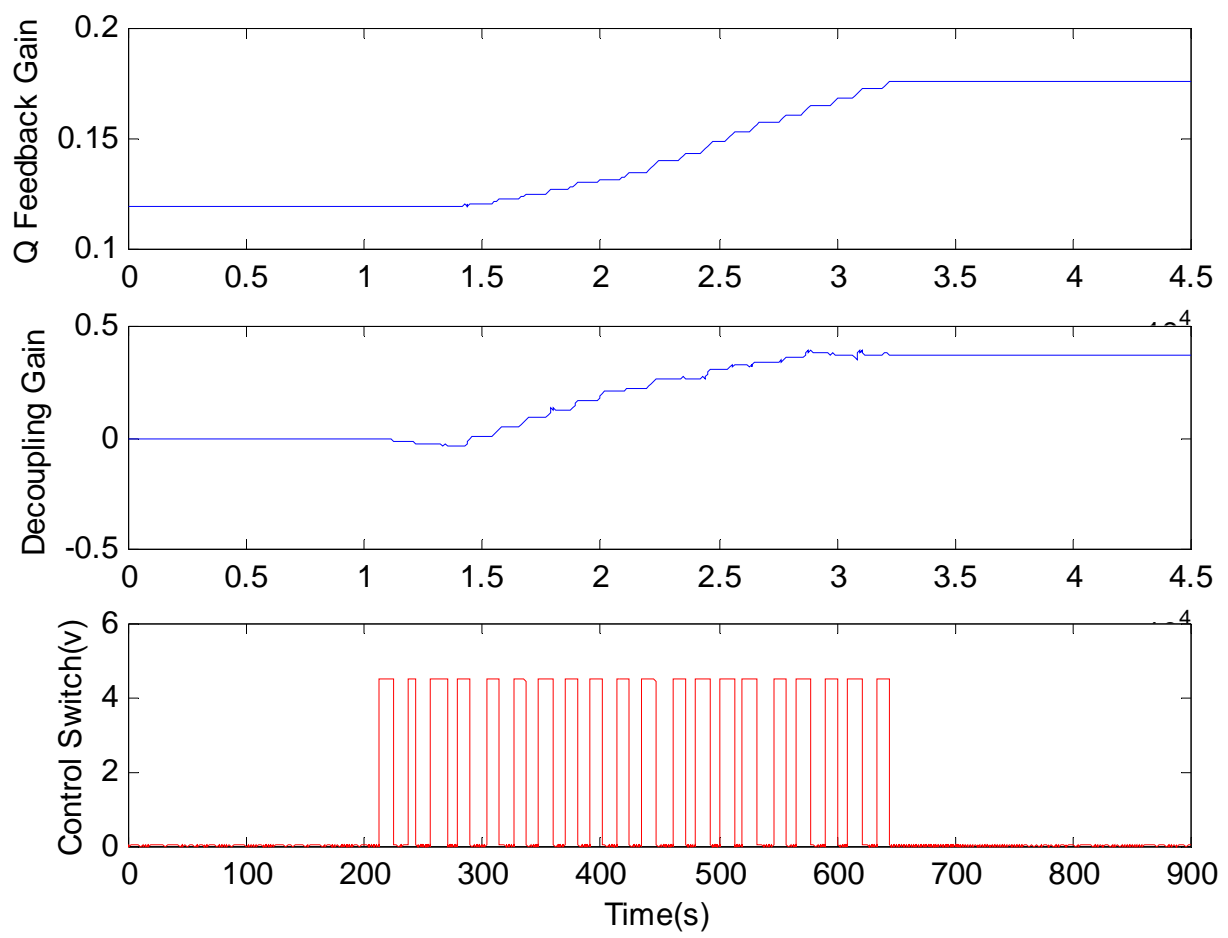

Figure 6-17 Feedback Gains

The learning process again, is demonstrated with the playback of the actual NN responses during the flight. Figures 6-18 and 6-19 illustrate the NN estimations and the gain updating process for the $11^{\text {th }}$ activation (434.66-447.02). The estimation from the on-line learning $\mathrm{NN}$ is shown with a solid line and a dotted line refers to reference estimation from the off-line learning NN. During the first four seconds, the on-line learning $\mathrm{NN}$ was trained with the real-time flight data. The estimation of the on-line learning $\mathrm{NN}$ is affected by the wind gust at this moment and was not used to update the controller gain. After four seconds, the on-line learning was deactivated and the estimation difference between the on-line and off-line NN was used to update the feedback controller gains. The pitch rate feedback gain starting from 0.1352 at the beginning of this activation and increased to a value of 0.1399 at the end (Figure 6-18). The roll-rate estimation difference from the on-line full $\mathrm{NN}$ observer and the off-line lateral-directional $\mathrm{NN}$ observer was used to update the decoupling feedback control gain. This decoupling gain was increased from 0.2222 to 0.2613 during this period (Figure 619). 

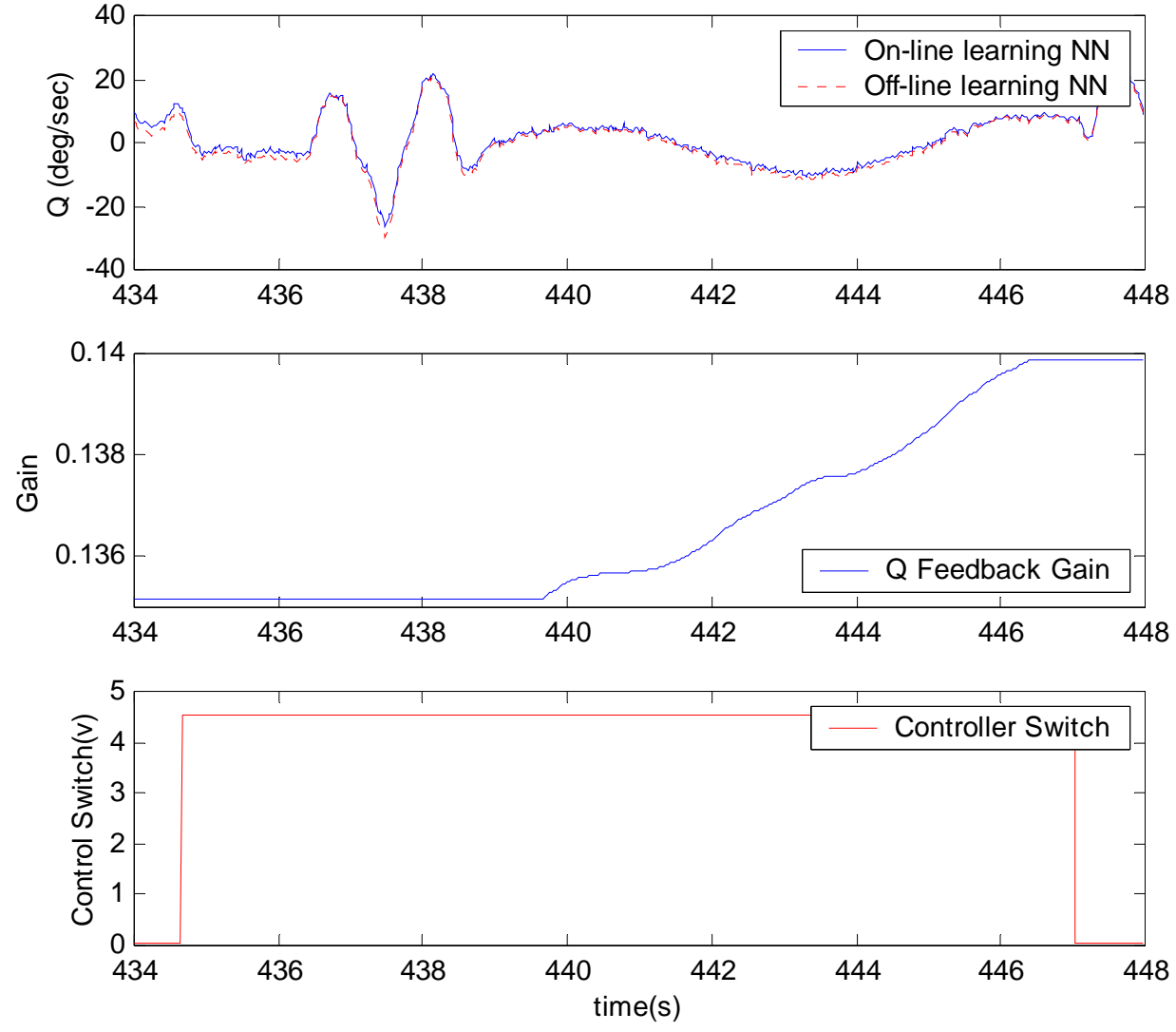

Figure 6-18 Pitch Rate Estimations 

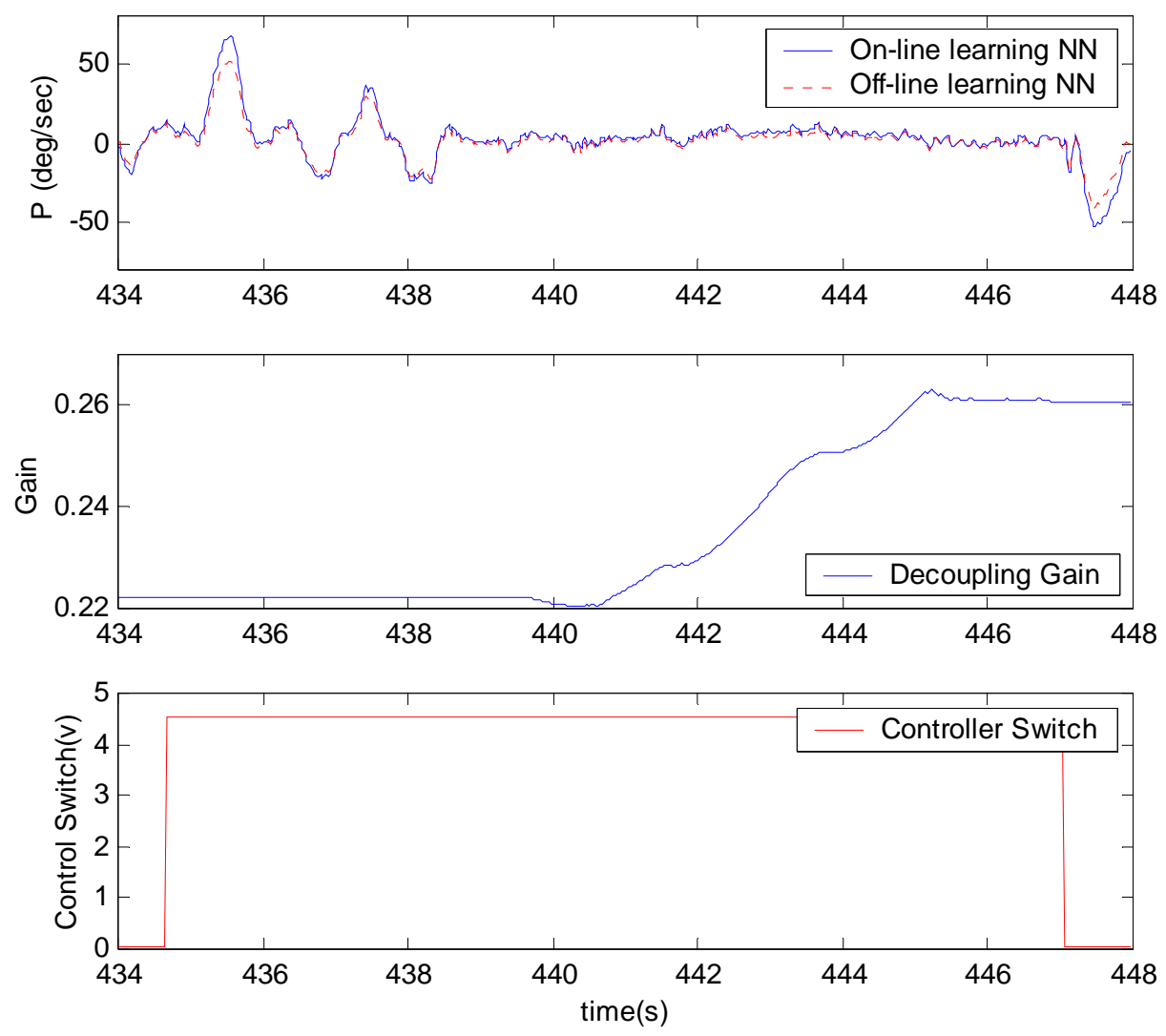

Figure 6-19 Roll Rate Estimations

Figure 6-20 shows the left elevator input, the roll rate response, the pitch rate response, and the angle of attack during the first activation (211.72- 224.78s) while the AFA controller just started to compensate for the failure. The circle indicates the four sec mark and the AFA controller was engaged after the mark. Figure 6-21 shows the same signals during the last $\left(20^{\text {th }}\right)$ activation (634.18- 644.98s), where the AFA controller almost finished the feedback gain updating process and provided a best possible accommodation for the right elevator failure. 


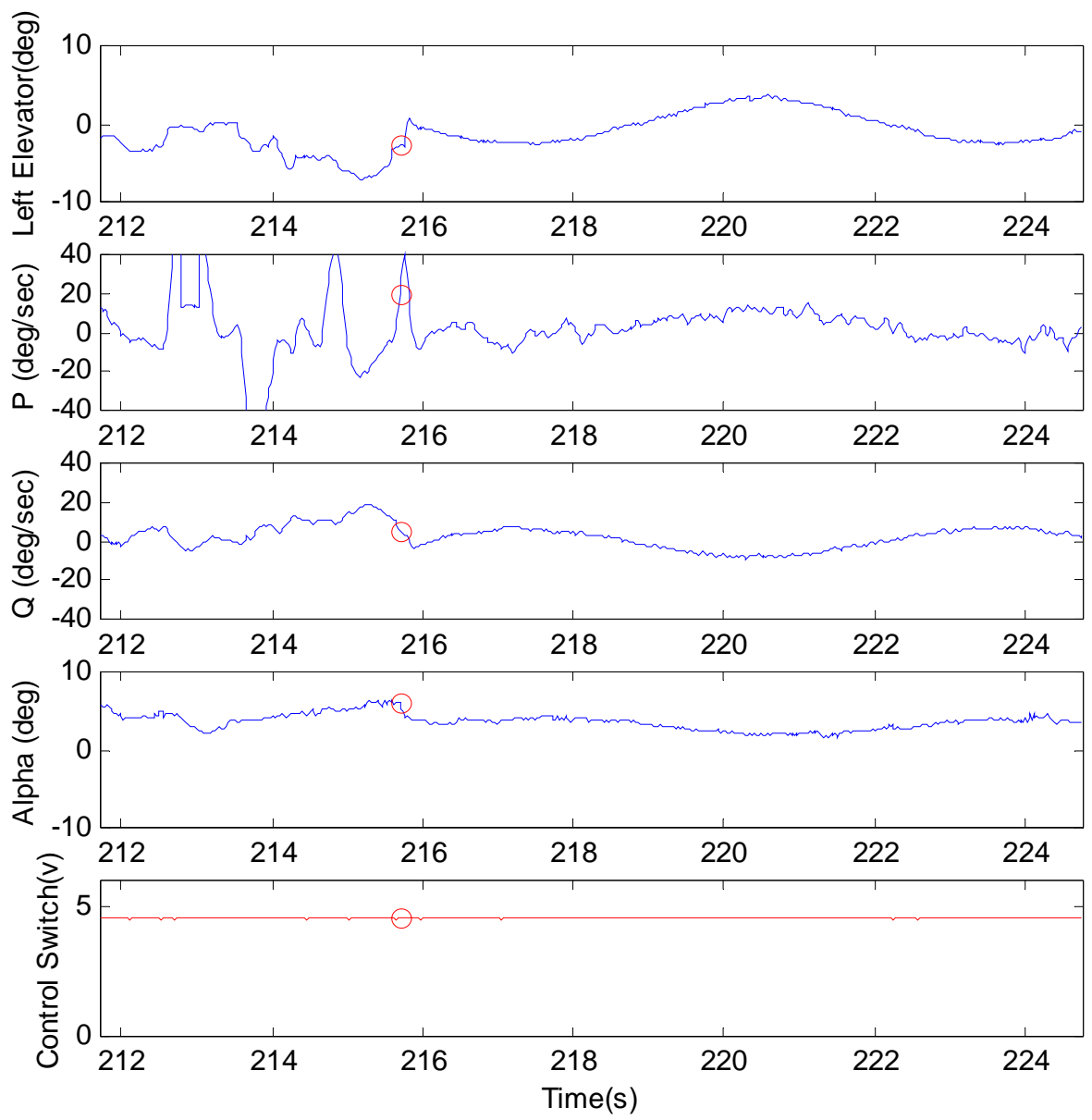

Figure 6-20 Aircraft Response (First Failure Activation) 

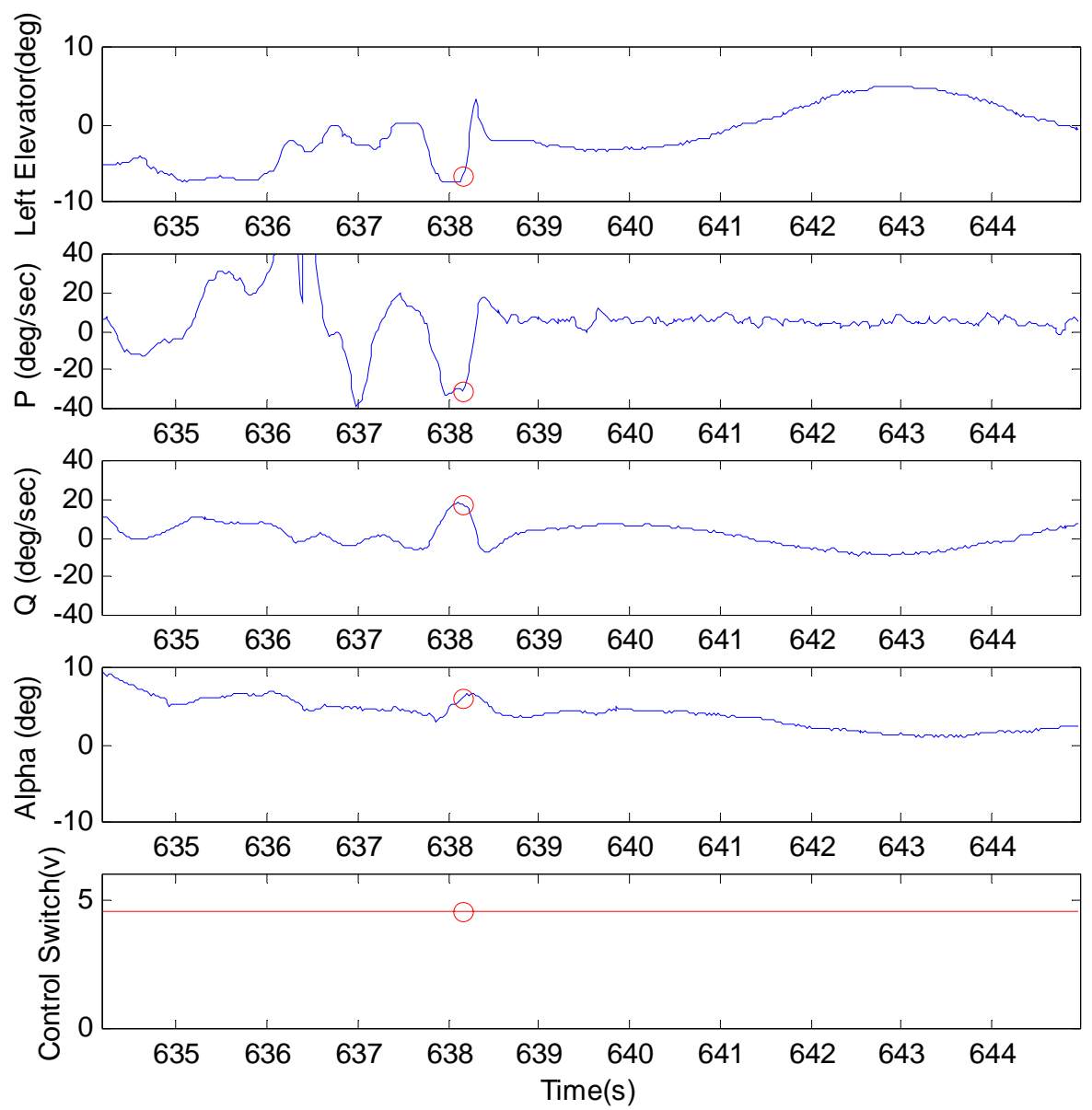

Figure 6-21 Aircraft Response (Last Failure Activation)

Again, to make it easier to compare, the elevator input and pitch rate response of the first and $20^{\text {th }}$ activation were plotted side by side in Figures 6-22 and 6-23 (data after the $4 \mathrm{sec}$ mark). The aileron inputs and roll rate response of the first and $20^{\text {th }}$ activation were also plotted in Figures 6-24 and 6-25. 


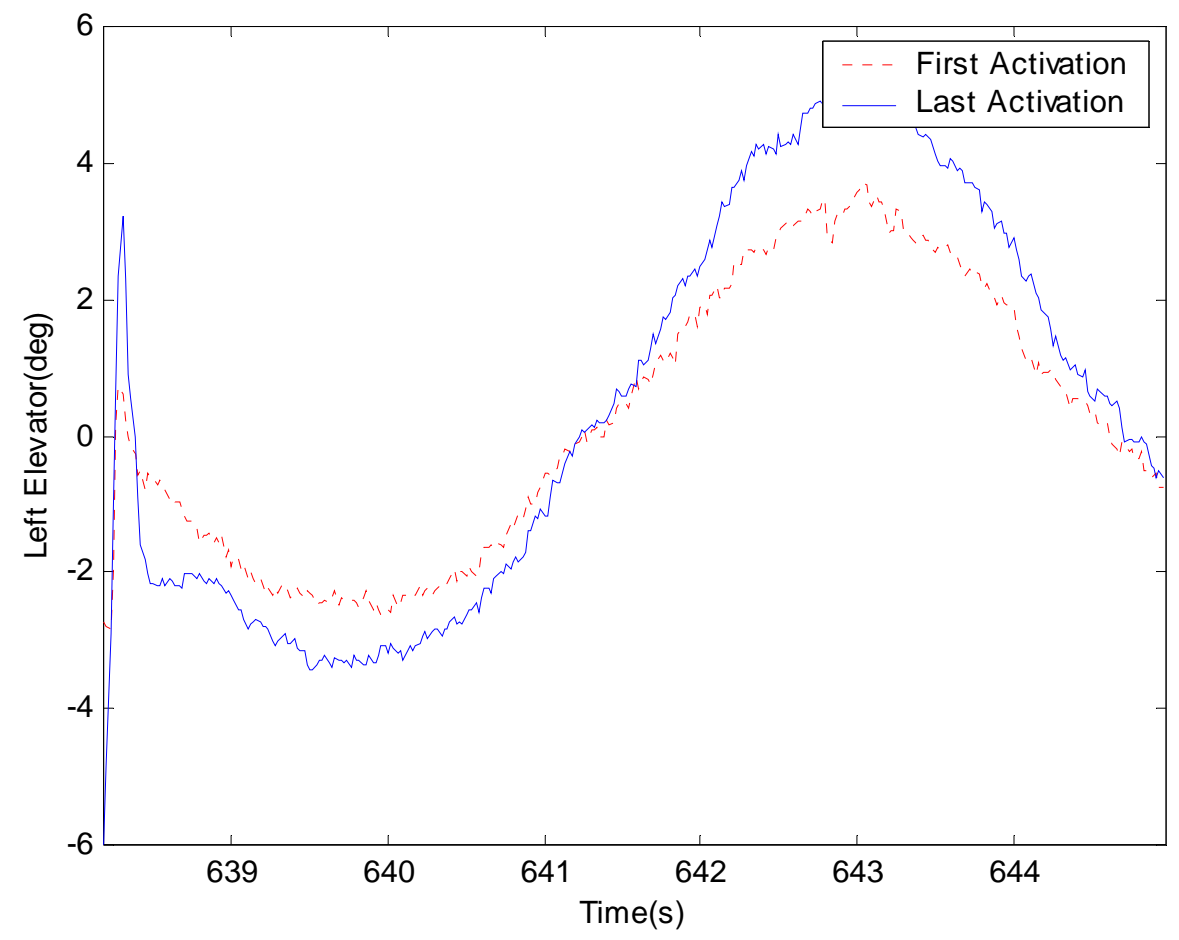

Figure 6-22 Left Elevator Deflections

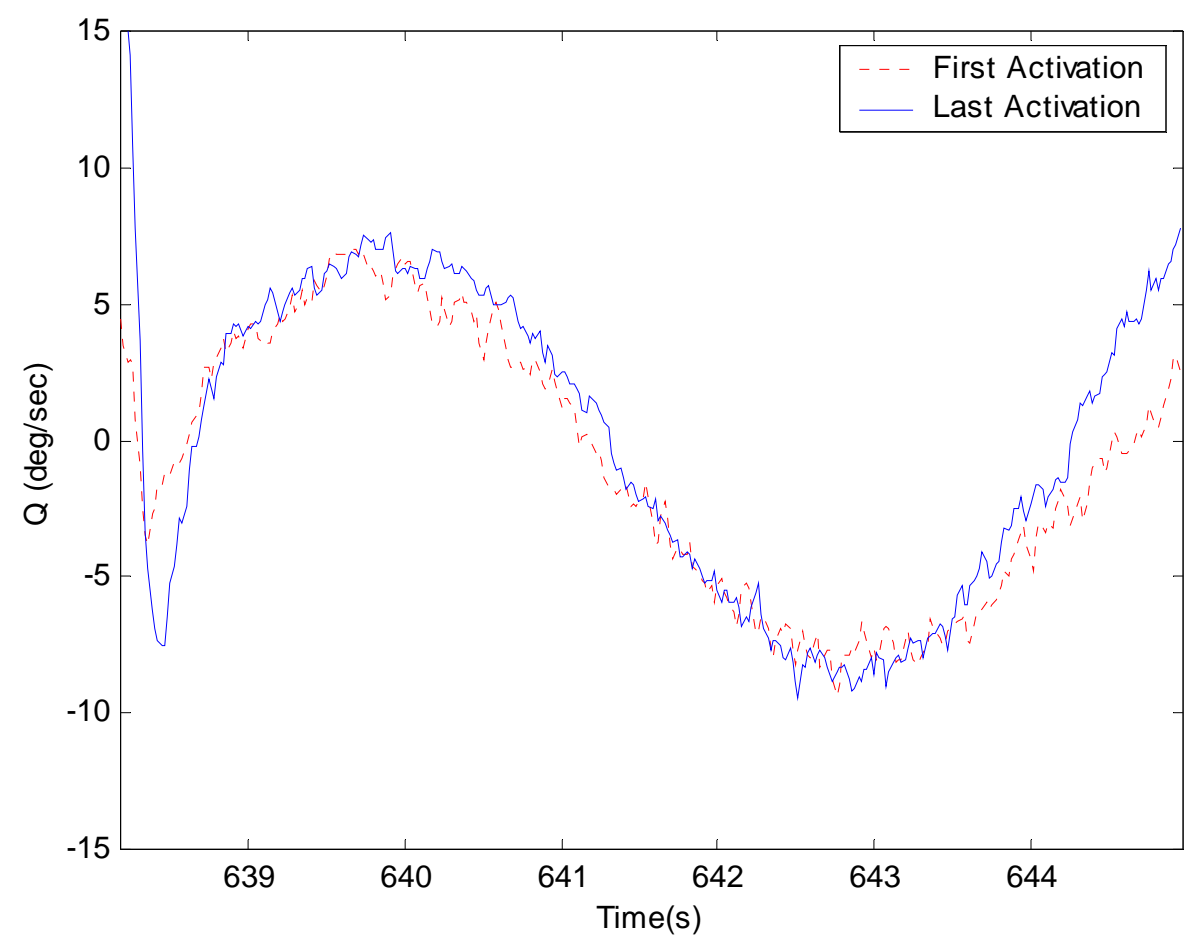

Figure 6-23 Pitch Rate 


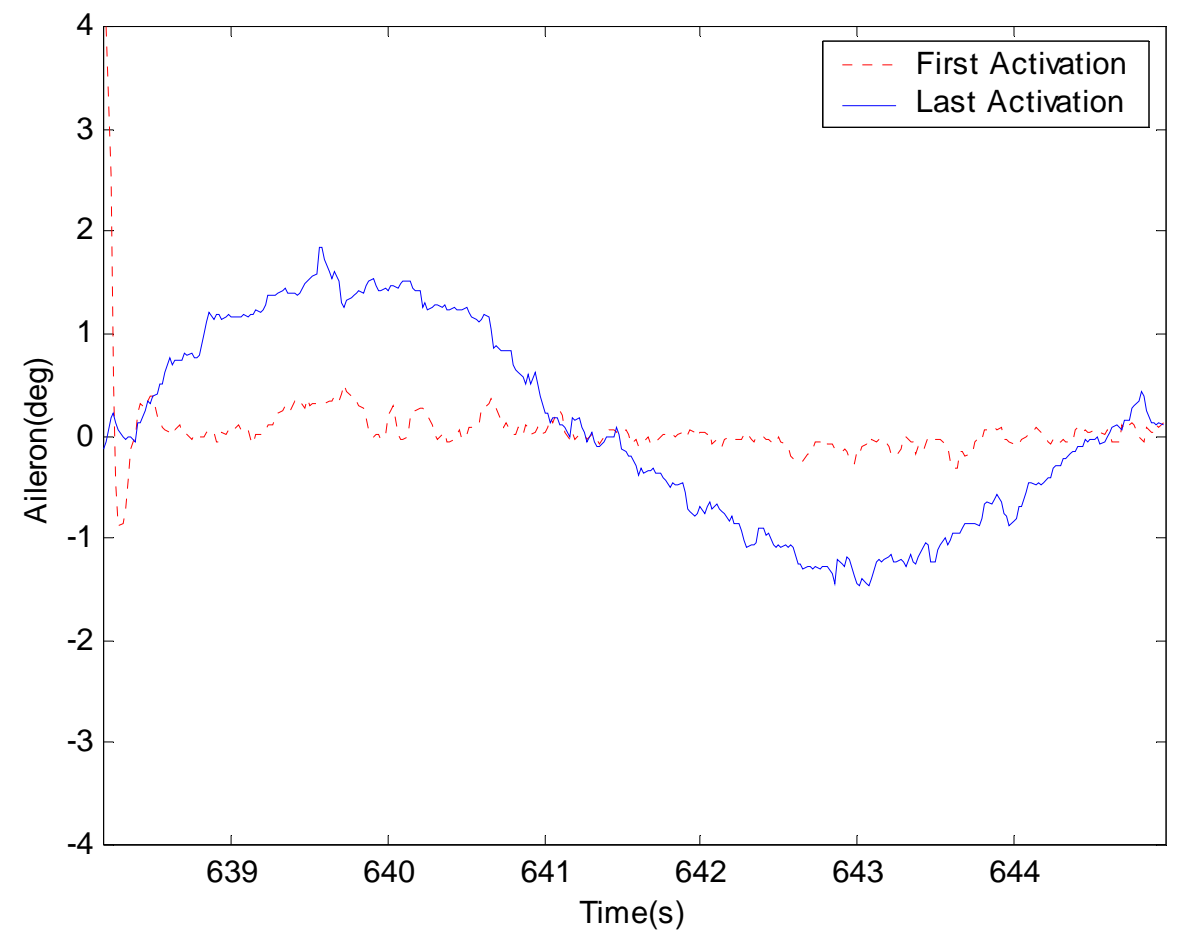

Figure 6-24 Aileron Deflections

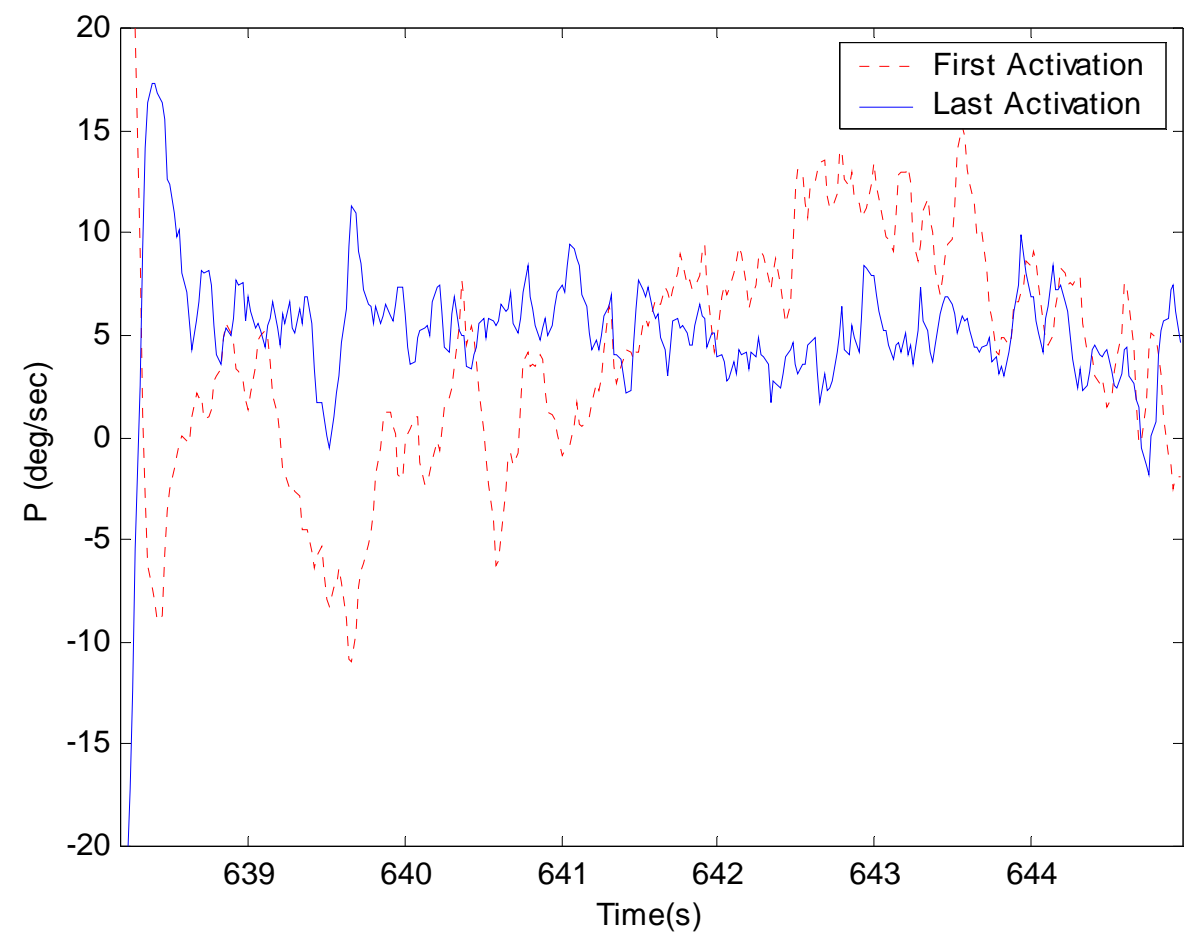

Figure 6-25 Roll Rate 
From these flight data, it is clearly shown that with the on-line NN learning and the updated AFA controller feedback gain, the control command on the left elevator had been increased to achieve an improved pitch rate response. The aileron inputs, starting at zero, were increased to compensate for the rolling moment caused by the single left elevator input. Figure 6-25 shows that with the AFA controller's compensation, the elevator inputs caused almost no response (fully compensated) on the aircraft lateral dynamics at the $20^{\text {th }}$ controller engagement. To further analysis the AFA controller accommodation process, the standard deviation of the left elevator deflection, aileron deflection, pitch rate and roll rate response of each controller switch activation are shown in Table 6-3:

\begin{tabular}{|c|c|c|c|c|}
\hline $\begin{array}{l}\text { Controller } \\
\text { Activation }\end{array}$ & $\begin{array}{l}\text { STD for Left } \\
\text { Elevator (deg) }\end{array}$ & $\begin{array}{c}\text { STD for } \\
Q(\text { deg/sec })\end{array}$ & $\begin{array}{l}\text { STD for Ailerons } \\
\text { (deg) }\end{array}$ & $\begin{array}{c}\text { STD for } \\
\text { R (deg/sec) }\end{array}$ \\
\hline 1 & 2.2073 & 5.4275 & 0.1567 & 5.9829 \\
\hline 2 & not enough data & not enough data & not enough data & not enough data \\
\hline 3 & 2.2486 & 5.4407 & 0.1372 & 6.1345 \\
\hline 4 & 2.3294 & 4.4155 & 0.1464 & 5.9952 \\
\hline 5 & 2.3495 & 4.9268 & 0.1904 & 6.0834 \\
\hline 6 & 2.3947 & 4.7619 & 0.2690 & 6.4022 \\
\hline 7 & 2.4266 & 4.5848 & 0.3433 & 5.7904 \\
\hline 8 & 2.5115 & 4.1943 & 0.3702 & 4.4762 \\
\hline 9 & 2.5803 & 4.4027 & 0.4811 & 4.6797 \\
\hline 10 & 2.4981 & 5.2231 & 0.5517 & 3.9276 \\
\hline 11 & 2.5257 & 5.3034 & 0.5711 & 3.4538 \\
\hline 12 & 2.5929 & 5.6817 & 0.6403 & 2.6342 \\
\hline 13 & 2.6482 & 5.9925 & 0.7260 & 2.9030 \\
\hline 14 & 2.8715 & 5.4687 & 0.8676 & 3.2038 \\
\hline 15 & 2.8006 & 5.9413 & 0.8593 & 2.7576 \\
\hline 16 & 2.8473 & 6.1183 & 0.9204 & 1.8089 \\
\hline 17 & 2.9323 & 5.5568 & 0.9850 & 2.3345 \\
\hline 18 & 2.9658 & 6.0300 & 1.0307 & 1.7396 \\
\hline 19 & 3.0576 & 5.6968 & 1.0376 & 1.4927 \\
\hline 20 & 3.0927 & 5.9357 & 1.0620 & 1.8474 \\
\hline
\end{tabular}

Table 6-3 Statistical Analysis - Elevator Failure 
Each of the STD value was calculated with 4 seconds of flight data after the actuator failure, which is the same time window as the one used for aileron failure analysis. Note that the second controller switch activation lasted only for 7.06 seconds, which does not contain enough information for analysis. This data point was abandoned in the analyzing procedure.

STD values from Table 6-3 were plotted in Matlab to facilitate the data analysis. Figure 6-26 shows the STD for the left elevator deflection and the pitch rate response, and Figure 6-27 shows the STD for the left aileron deflection and the roll rate response.
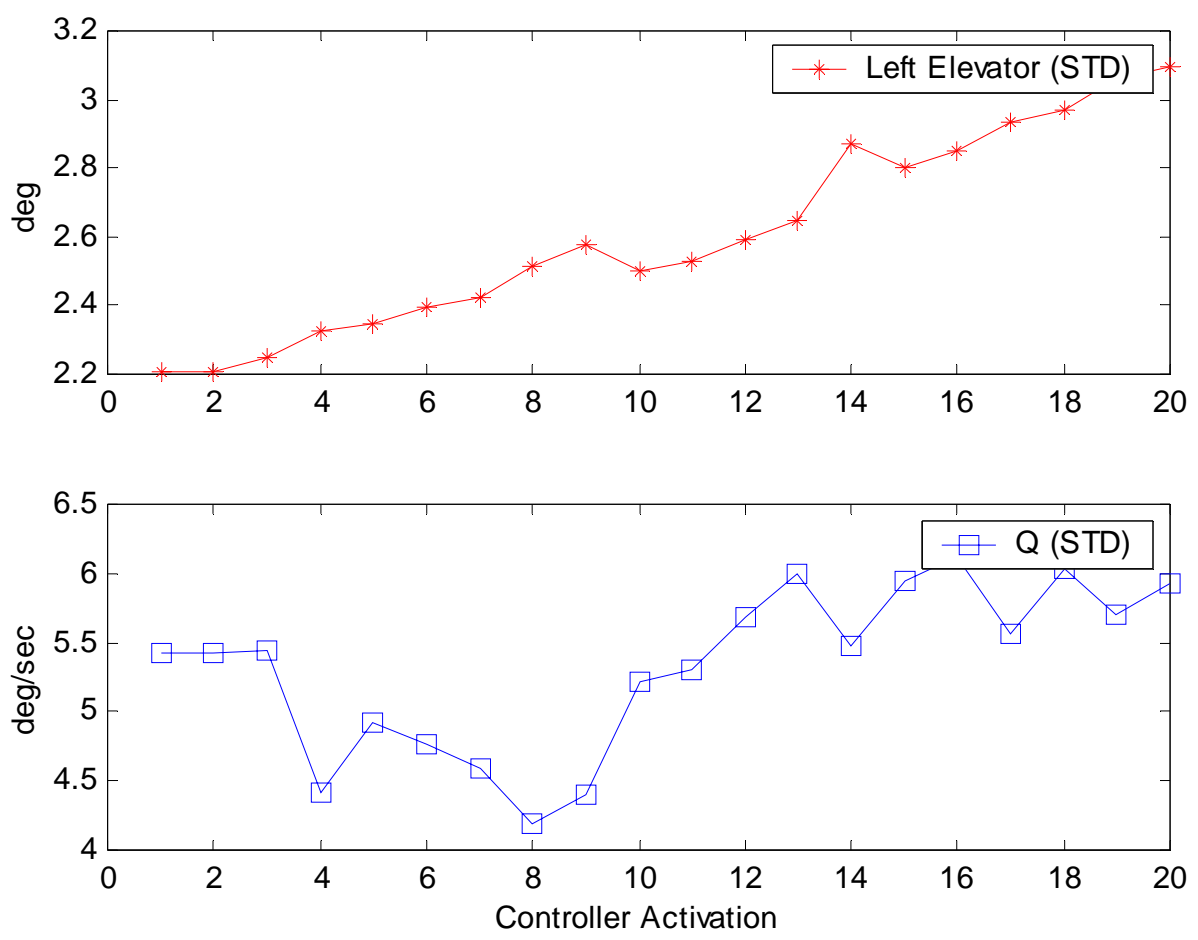

Figure 6-26 Elevator Failure Statistical Analysis -Longitudinal 

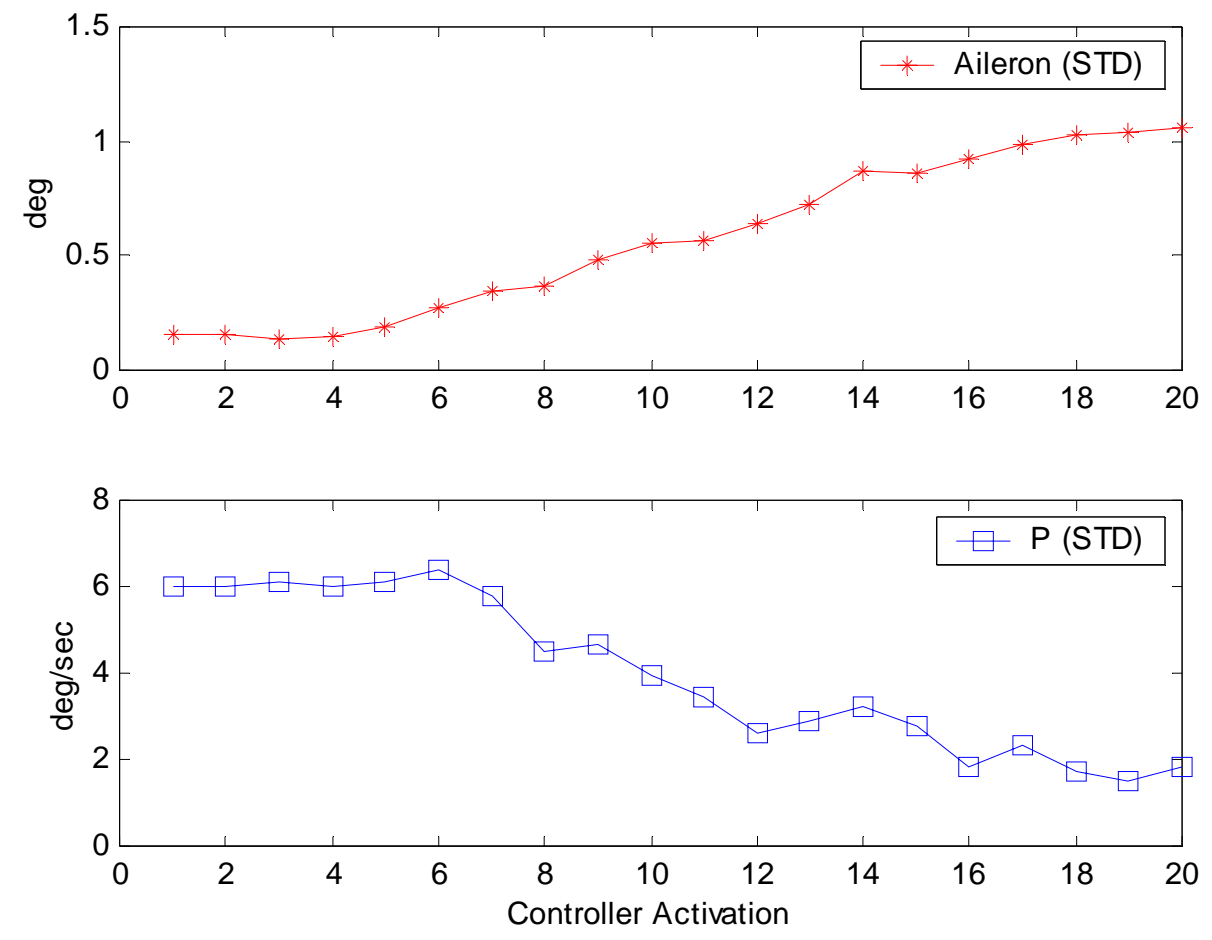

Figure 6-27 Elevator Failure Statistical Analysis -Lateral

From these plots, it can be observed that: with the $\mathrm{NN}$ on-line learning, the designed AFA controller gradually increased the left elevator control command to compensate for the loss of right elevator. At the same time, the aileron command was increased to compensate for the rolling moment caused by the left elevator deflection. In this way, the designed AFA controller accommodated for the negative effect caused by the right elevator failure and improved aircraft handling qualities.

A performance comparison was also performed with three flight conditions:

- Linear controller at nominal flight condition (no elevator failure)

- Linear controller with right elevator failure

- AFA controller with right elevator failure

Flight data collected from flight testing Phase 4 and Phase 5 was used in this comparison. The left elevator deflections for three scenarios are shown in Figure 6-28 and the corresponding pitch rate responses are shown in Figure 6-29. The aileron deflections are shown in Figure 6-30; with aircraft roll rate responses shown in Figure 631. 


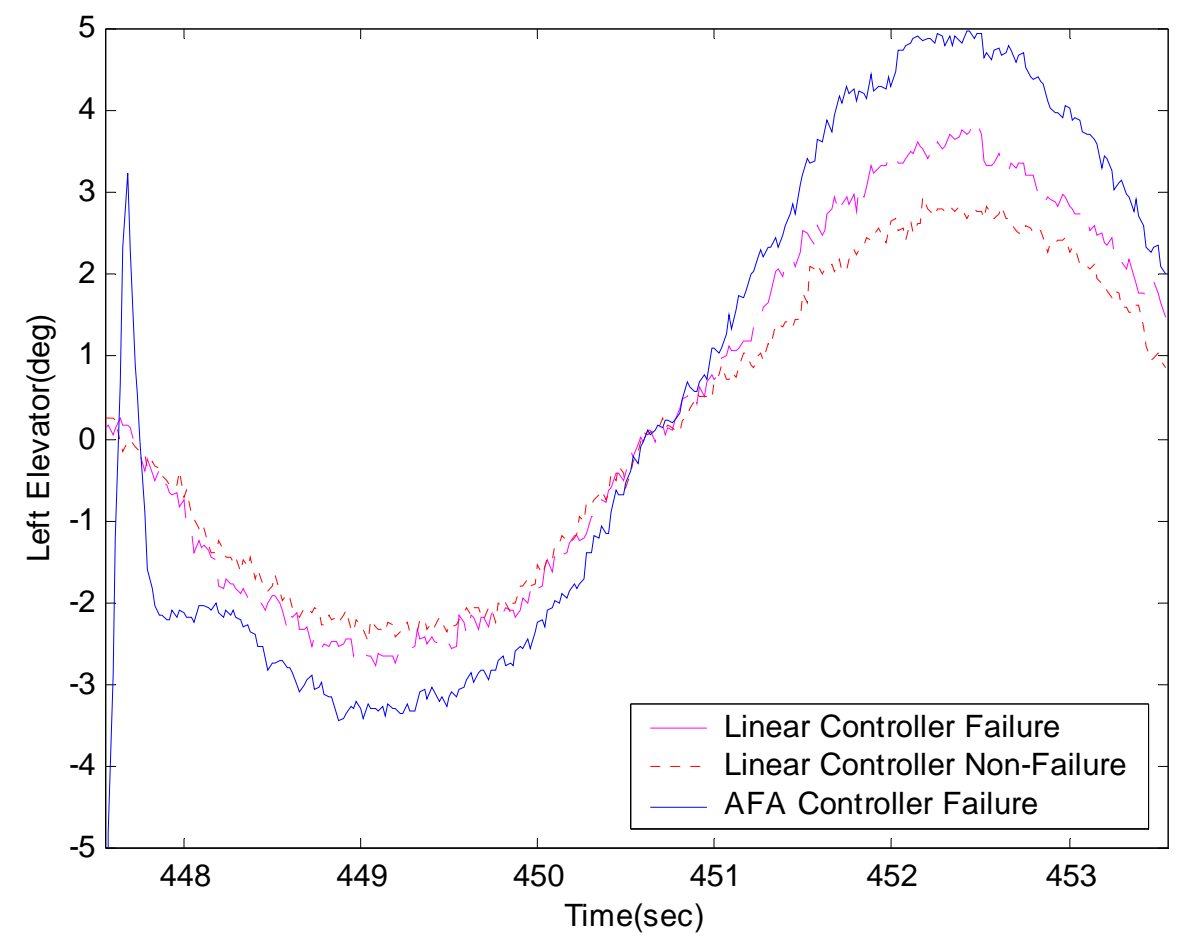

Figure 6-28 Performance Comparison - Left Elevator

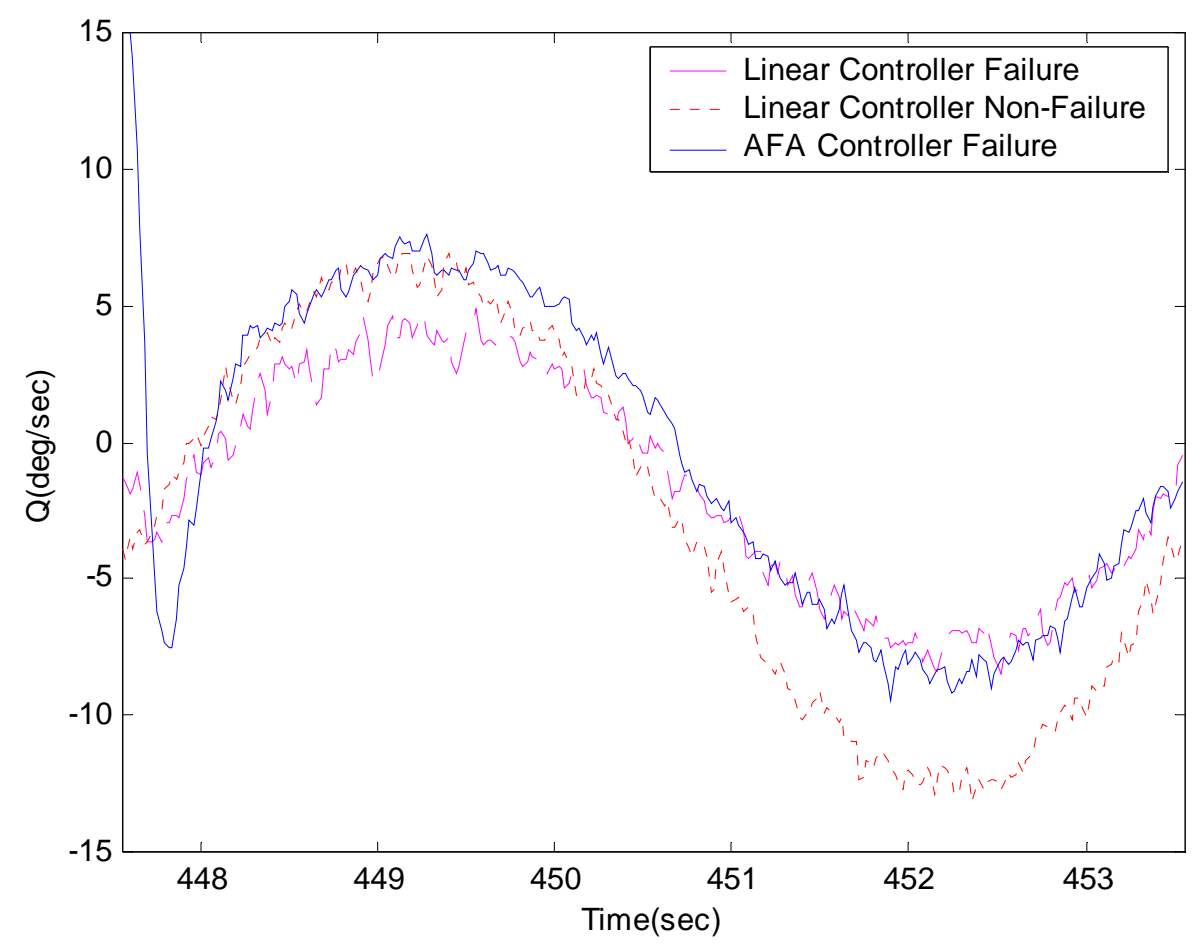

Figure 6-29 Performance Comparison - Pitch Rate 


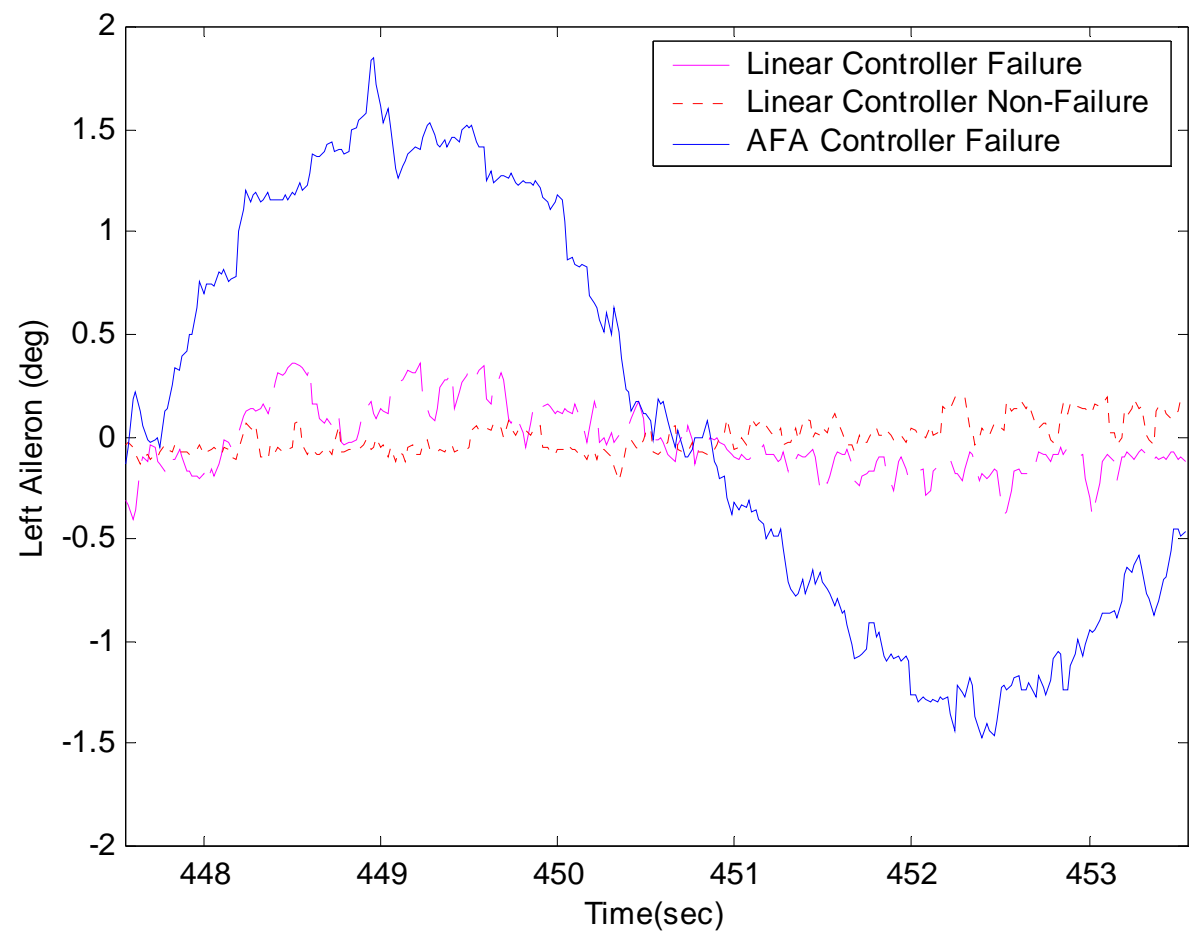

Figure 6-30 Performance Comparison - Left Aileron

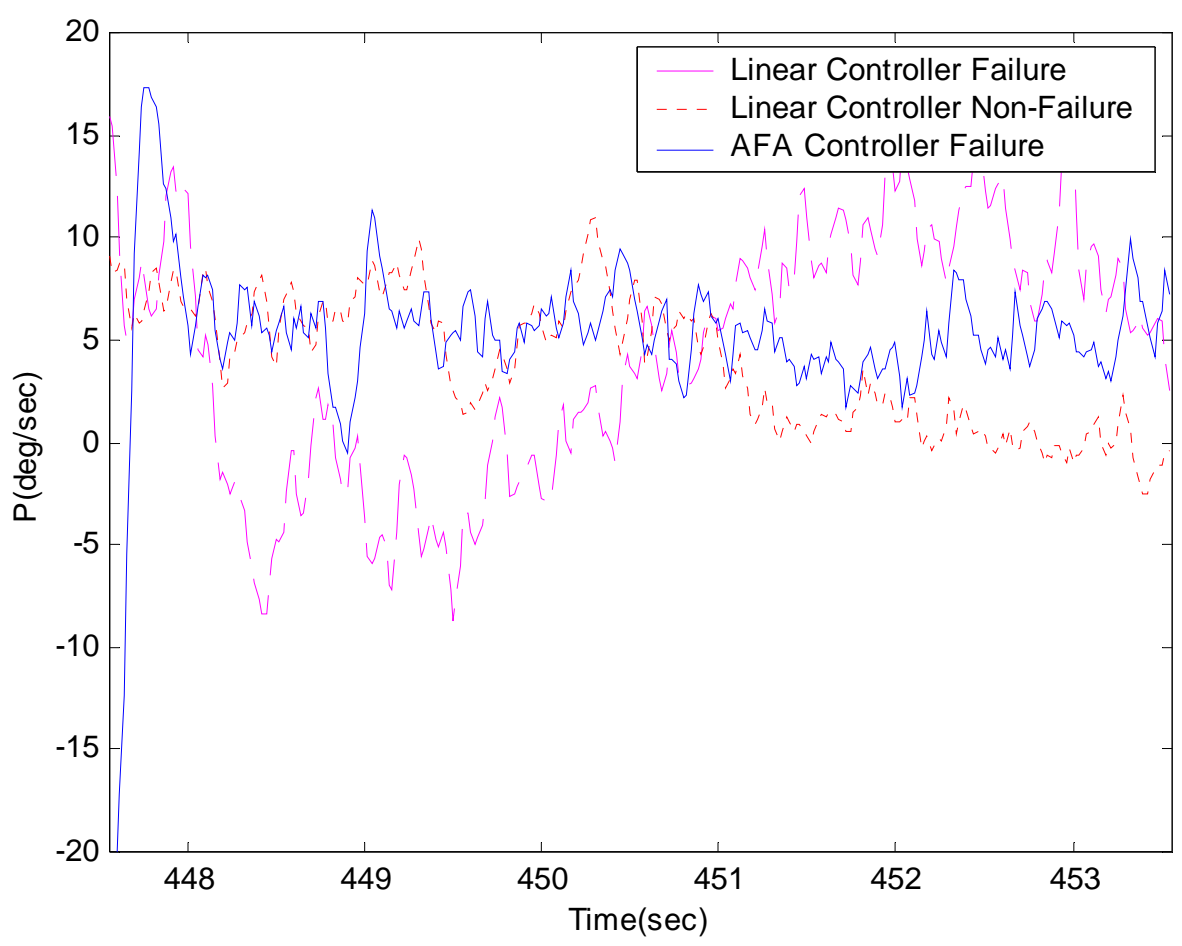

Figure 6-31 Performance Comparison - Roll Rate 
With the AFA controller been activated, the left elevator control command had been increased greatly compared to the linear controller commands. Control commands were also sent to ailerons to cancel out the coupling between the single left elevator deflection and aircraft lateral dynamics. The STD of the left aileron deflection, pitch rate response, left aileron deflection, and roll rate response are listed in Table 6-4:

\begin{tabular}{|l|c|c|c|}
\hline & $\begin{array}{c}\text { Linear Controller } \\
\text { No Failure }\end{array}$ & $\begin{array}{c}\text { Linear Controller } \\
\text { Elevator Failure }\end{array}$ & $\begin{array}{c}\text { AFA Controller } \\
\text { Elevator Failure }\end{array}$ \\
\hline STD for Left Elevator (deg) & 1.8405 & 2.2360 & 3.1129 \\
\hline STD for Q (deg/sec) & 6.7681 & 4.0535 & 5.9458 \\
\hline STD for Left Aileron (deg) & 0.0804 & 0.1687 & 1.0134 \\
\hline STD for P (deg/sec) & 3.2148 & 6.1106 & 4.8164 \\
\hline
\end{tabular}

Table 6-4 Performance Comparison - Three configurations

From these plots and calculated STD values, it is clearly shown that the AFA controller improved the aircraft's pitch rate response and canceled out the rolling moment caused by one side elevator. In this way, the designed AFA controller compensated for the elevator failure and provided a performance very close to the linear controller under the normal flight condition. 


\section{Chapter 7 Conclusions and Recommendations}

This project successfully designed, implemented, and flight-tested an AFA control scheme which can compensate for an aircraft with actuator failure. Two-failure scenarios were studied, simulated and flight-tested:

Failure 1: Right aileron locked at the trim position

Failure 2: $\quad$ Right elevator locked at the trim position.

Neural networks were selected in the controller design for their learning ability and nonlinearity. An actuator failure accommodation controller scheme was designed and developed. On-board hardware and software were tailored to implement the AFA controller scheme into the WVU YF-22 research UAVs. A set of flight tests from aircraft assessment, data acquisition, failure analysis, to a final demonstration of the AFA control scheme had been successfully completed. The flight testing data shows a satisfactory performance of the AFA controller matching the results of simulation study.

From this research effort, further work with the actuator failure accommodation could be pursued in several ways. With caution, different configurations of actuator failures could be tested including the locking of one control surface at positions other than the trim. Different types of NN learning algorithms could be tested to minimize the learning procedure. However, this type of study will have a much higher requirement on the on-board computer's CPU speed. Additional research may also be pursued towards creating a more robust system to handle wind gust disturbances, which was a major difficulty for flight testing a NN-based controller on a small UAV. Furthermore, the stability analysis and validation of the control system posts a large challenge, which is a common and widely recognized problem in the NN control community, 


\section{References}

[1] Perhinschi, M.G, Lando, M., Massotti, L., Campa, G., Napolitano, M.R., Fravolini, M.L., "On-Line Parameter Estimation Issues for the NASA IFCS F-15 Fault Tolerant Systems"

[2] Jong-Yeob Shin, N. Eva Wu "Linear Parameter Varying Control for Actuator Failure”. NASA ICASE report, No.2002-34

[3] Kline-Schoder, R.,Rauch, H., Youssef, "Fault Detection, Isolation, and Reconfiguration for Aircraft Using Neural Networks," Proceedings of the AIAA Guidance, Navigation and Control Conference, AIAA Paper 93-3876, Monterey, Ca, 1993.

[4] Huang, C., Tylock, J., Engel, S., Whitson, J., Eilbert, J., "Failure-Accommodating Neural Network Flight Control," Proceedings of the AIAA Guidance, Navigation and Control Conference, AIAA Paper 92-4394, HiltonHead, SC, 1992.

[5] Napolitano, M.R., Chen, C.I.,et. al., "Aircraft Failure Detection and Identification Using Neural Networks," Journal of Guidance, Control, and Dynamics, Vol. 16, No. 6, pp.999 1009, 1993.

[6] M.T.Hagan, H.B.Demuth, "Neural Networks for Control" Proceeding of the American Control Conference. June 1999

[7] Tianping Chen Hong Chen, "Universal approximation to nonlinear operators by neural networks with arbitrary activation functions and its application to dynamical systems", IEEE Transactions on Neural Networks Jul 1995

[8] John Kaneshige, John Bull. "Generic Neural Flight Control and Autopilot System". AIAA-2000-4281

[9] W.T.Miller, "Sensor-Based Control of Robotic Manipulators Using a General Learning Algorithm”. IEEE Journal of Robotics and Automation. Vol.3, no.2. 1987

[10] I. Rivals and L. Personnaz. "Internal Model Control Using Neural Networks." Proceedings of the IEEE International Symposium on Industrial Electronics, june 1996

[11] W. B. Dunbar , M. B. Milam. "Model Predictive Control of a Thrust-Vectored Flight Control Experiment”. IFAC02 
[12] G.G. Lendaris, R. Santiago, "Controller Design via Adaptive Critic and Model Reference Methods". IJCNN 2003

[13] A. Gupta, S.N.Balakrishnan. "Development and Implementation of Adaptive Critic Based Optimal Neurocontroller on a Cantilever Plate". Proceeding of the American Control Conference. June 1999

[14] G. Wyeth, G. Buskey "Flight Control Using an Artificial Neural Network". Proceedings of the Australian Conference on Robotics and Automation (ACRA 2000)

[15] Eric N. Johnson, Anthony J. Calise. "Feedback Linearization with Neural Nwtwork Augmentation Applied to X-33 Attitude Control". AIAA-2000-4157

[16] E.N. Johnson, A.J.Calise "Adaptive Guidance and Control for Autonomous Launch Vehicles” In IEEE Aerospace Conference, April 2001.

[17] E.N. Johnson, A.J.Calise "Reusable Launch Vehicle Adaptive Guidance and Control Using Neural Networks" AIAA Guidance, Navigation and Control Conference, number 4381, 2001.

[18] J. Eric Corban, Anthony J. Calise. "Flight Test of an Adatptive Control System For Unmanned Helicopter Trajectory Following". AIAA-2000-4058

[19] E.N. Johnson, A.J.Calise, "Pseudo-Control Hedging: A New Method for Adaptive Control" Workshop on Advances in Guidance and Control Technology, 2000.

[20] Thompson,P.M, Owens, K.J. "Assessment of Feedforward Neuro-Controllers Using Describing Functions", AIAA-97-3762

[21] Asriel U. Levin, Kumpati S.Narendra. "Control of Nonlinear Dynamical Systems Using Neural Networks: Controllability and Stabilization" IEEE Transactions of neural networks, VOL.4 NO.2 march 1993

[22] Y.Zhang, P.Peng "Stable Neural Controller Design for Unknown Nonlinear Systems Using Backstepping"

[23] J.B.Dugan, J.W. Watterson. "Reliability Analysis of Artifical Neural Networks" Proceeding of the American control conference. June 1999

[24] F.Ahmed-Zaid, P.A.Ioannou, "Identification and Control of Aircraft Dynamics Using Radial Basis Function Neural Networks". AIAA-92-4393-CP 
[25] Napolitano, M.R., Molinaro, G., Innocenti, M., Seanor, B., Martinelli, D., "A Complete Hardware Package for a Fault Tolerant Flight Control System Using On-Line Learning Neural Networks", Proceedings of the American Control Conference (ACC) 1999, San Diego, CA, June 1999

[26] Brinker, J., and Wise K. "Flight testing of a reconfigurable flight control law on the X-36 tailless fighter aircraft" AIAA Guidance, Navigation, and Control Conference. 2000

[27] Air Force Research Laboratory, "First Flight Test Demonstration of Neural Network Software", http://www.afrlhorizons.com/Briefs/0001/VA9904.html

[28] Dryden Flight Research Center, "X-36 Tailless Fighter Agility Research Aircraft in flight”, http://www.dfrc.nasa.gov/Gallery/Photo/X-36/HTML/EC97-442946.html

[29] Dryden Flight Research Center, "X-36 transfers to Dryden", http://www.dfrc.nasa.gov/Newsroom/X-Press/1998/Dec15/x36.html

[30] NASA Dryden Flight Research Center, "Intelligent Flight Control Systems", http://www.dfrc.nasa.gov/Newsroom/FactSheets/FS-076-DFRC.html

[31] Perhinschi M. G., Napolitano M.R., Campa G., Seanor B., Gururajan S., "Design of Intelligent Flight Control Laws for the WVU F-22 Model Aircraft", Proceedings of the AIAA Intelligent Systems Technical Conference 2004 Chicago IL, AIAA2004-6282

[32] Perhinschi M. G., Napolitano M.R., Campa G., Burke H. E., Larson R. R., Burken J., Fravolini M. L., "Design and Testing of a Safety Monitor Scheme on the NASA Gen_2 IFCS F-15 Flight Simulator", Proceedings of the AIAA Intelligent Systems Technical Conference 2004 Chicago IL, AIAA2004-6284

[33] Perhinschi M. G., Burken J., Napolitano M.R., Campa G., Fravolini M. L.,"Performance Comparison of Different Neural Augmentation for the NASA Gen_2 IFCS F-15 Control Laws", Proceedings of the American Control Conference 2004 Boston MA, pp3180-3184

[34] Perhinschi M. G., Napolitano M.R., Campa G., Fravolini M. L., "Integration of Fault Tolerant System for Sensor and Actuator Failures within the WVU NASA 
F-15 Simulator", Proceedings of the AIAA Guidance, Navigation, and Control Conference, August 2003, Austin, Texas, AIAA-2003-5643

[35] Perhinschi M. G., Napolitano M.R., Stolarik B., Hammaker S., Campa G., Rogers S., "Design Of Safety Monitor Schemes for a Fault Tolerant Flight Control System", Proceedings of the AIAA Guidance, Navigation, and Control Conference, August 2003, Austin, Texas, AIAA-2003-5646

[36] Battipede M., Gili P., Lando M, Napolitano M. R., Perhinschi M. G., Campa G., "Comparative Analysis of Neural Control Systems Within the NASA IFCS F-15 WVU Simulator", Proceedings of the AIAA Guidance, Navigation, and Control Conference, August 2003, Austin, Texas, AIAA-2003-5643

[37] Perhinschi M. G., Napolitano M.R., Campa G., Fravolini M. L., Massotti L.,Lando M., "Augmentation of a Non Linear Dynamic Inversion Scheme Within the NASA IFCS F-15 WVU Simulator", Proceedings of the American Control Conference 2003, June 4-6, 2003 Denver CO, USA, pp1667-1672

[38] Perhinschi M. G., Campa G., Napolitano M.R., Fravolini M. L., Lando M., Massotti L., "Performance Comparison of Fault Tolerant Control Laws Within the NASA IFCS F-15 WVU Simulator", Proceedings of the American Control Conference 2003, June 4-6, 2003 Denver CO, USA, pp1661-1666

[39] Battipede M., Gili P., Napolitano M. R., Perhinschi M. G., Massotti L., Lando M., "Implementation of an Adaptive Predictor-Corrector Neural Controller within the NASA IFCS F-15 WVU Simulator", Proceedings of the American Control Conference 2003, June 4-6, 2003 Denver CO, USA, pp1302-1307

[40] Perhinschi M. G., Lando M., Massotti L., Fravolini M. L., Campa G., Napolitano M.R., "On-Line Parameter Estimation for Real Time Application for the NASA IFCS F-15 Fault Tolerant Systems", Proceedings of the American Control Conference, Anchorage, AK, 2002, pp191-196

[41] Maureen Caudill, "Neural Network Primer: Part I" AI Expert, Feb. 1989

[42] Widrow, B. and Hoff, M. E. "Adaptive switching circuits" IRE WESTCON Connection Record, volume 4, pages 96-104.

[43] Roskam, Jan, "Airplane Flight Dynamics and Automatic Flight Controls, Part I", Design, Analysis and Research Corporation, Lawrence, Kansas, 1995 
[44] Stevens, B.L., Lewis, F.L., “Aircraft Control and Simulation”, John Wiley and Sons, New York, 1992.

[45] Haykin, S., "Neural Networks: A comprehensive Foundation", McMillan College Publishing Company, Inc., 1994.

[46] Motyka, P., Bonnice, W., Hall, S., Wagner, E. "The Evaluation of Failure Detection and Isolation Algorithms for Restructurable Control", NASA Contractor Report 177983, 1985

[47] Kerr, T., "Decentralized Filtering and Redundancy Management/Failure Detection for Multisensor Integrated Navigation Systems," IEEE Transactions on Information Theory, pp.191 208, 1986.

[48] Willsky, A.S., "A Survey of Design Methods for Failure Detection in Dynamic Systems," Automatica, Vol. 12, pp.601 611, 1976.

[49] Willsky, A.S., "Failure Detection in Dynamic Systems," Agard LS-109, Neuilly sur Seine, France, pp.2.1 2.14, 1980.

[50] Petri A. Jokinen, "Comparison of Neural Network Models for Process Fault Detection and Diagnosis Problems," Proceedings of the International Joint Conference on Neural Networks, pp.I-239 I-244, 1991.

[51] R. Sreedhar, B. Fernandez, C.Y. Masada, “A Neural Network Based Adaptive Fault Detection Scheme," Proceedings of the American Control Conference, pp.3259 3263, 1995.

[52] Narendra, K.S., Partasarathy, K. "Identification and Control of Dynamical Systems Using Neural Networks", IEEE Transactions on Neural Networks, Vol. 1, No 1, pp.4 27, 1990.

[53] Levin, A.U., Narendra, K.S. "Control of Non-Linear Dynamical Systems Using Neural Networks: Controllability and Stabilization”, IEEE Transactions on Neural Networks, Vol.4, No.2, pp.192 206, 1993.

[54] M.M. Polycarpou, A.T. Vemuri, "Learning Methodology for Failure Detection and Accommodation," IEEE Control Systems, pp.16 24, 1995.

[55] M.M. Polycarpou, A.J. Helmicki, "Automated Fault Detection and Accommodation: A Learning Systems Approach," IEEE Transactions on Systems, Man, and Cybernetics, Vol.25, No.11, pp.1447 1458, 1995. 
[56] C.M. Ha, "Neural Networks Approaches to AIAA Aircraft Control Design Challenge," Journal of Guidance, Control, and Dynamics, Vol. 18, No. 4, pp. 731 739, 1995.

[57] Ha, C.M., Wei, Y.P., Bessolo, J.A., "Reconfigurable Aircraft Flight Control System Via Neural Networks," Proceedings of the 1992 Aerospace Design Conference, AIAA Paper 92-1075, Irvine, Ca, 1992.

[58] Chi-Yuan Chiang, H.M. Youssef, "Neural Network and Fuzzy Logic Approach to Aircraft Reconfigurable Control Design," Proceedings of the American Control Conference, pp.3505 3509, 1995.

[59] Napolitano, M.R., Casdorph, V.,Neppach, C., Naylor, S, “On-line Learning Neural Architectures and Cross-Correlation Analysis for Actuator Failure Detection and Identification," International Journal of Control, Vol.63, No.3, pp.433 455, 1996.

[60] Napolitano, M.R., Steve Naylor et. al., "On-Line Learning Nonlinear Direct Neurocontrollers for Restructurable Systems," Journal of Guidance, Control, and Dynamics, Vol. 18, No. 1, pp.170 176, 1995.

[61] Napolitano, M.R., Neppach, C. et. al., "Neural-Network-Based Scheme for Sensor Failure Detection, Identification, and Accommodation," Journal of Guidance, Control, and Dynamics, Vol.18, No.6, pp.1280 1286, 1995.

[62] Napolitano, M.R., Swaim, R.L., "New Technique for Aircraft Flight Control Reconfiguration," Journal of Guidance, Control, and Dynamics, Vol. 14, No.1, pp.184 190, 1991.

[63] Napolitano, M.R., An,Y., Seanor, B., "A fault tolerant flight control system for sensor and actuator failures using neural networks", Aircraft Design Journal, Vol.3, pp. 103-128.

[64] An, Y., "A Design of Fault Tolerant Flight Control Systems for Sensor and Actuator Failures Using On-Line Learning Neural Networks", Dissertation, WVU, Mechanical and Aerospace Dept., Morgantown, WV, 1998.

[65] Del Gobbo, Napolitano, M.R., "Experience in Developing System Requirements Specification for a Sensor Failure Detection and Identification Scheme", 
Proceedings of the 3rd IEEE High-Assurance Systems Engineering Symposium, Washington DC, November 1998.

[66] Del Gobbo, D., Cukic, B., Easterbrook, S., Napolitano, M.R., "Fault Detectability Analysis for Requirements Validation of Fault Tolerant Systems", Proceedings of the 4th IEEE High-Assurance Systems Engineering (HASE) Symposium, Washington DC, November 1999

[67] Napolitano, M.R., Younghwan, A., Seanor, B., Pispistos, S., Martinelli, D., "Application of a Neural Sensor Validation Scheme to Actual Boeing B777 Flight Data", Proceedings of the 1999 AIAA Guidance Navigation \& Control Conference, August 1999

[68] Alexander, C., Cortellessa, V., Del Gobbo, D., Mili, A., Napolitano, M.R., "Modeling the Fault Tolerant Capability of a Flight Control System: An Exercise in SCR Specification", Proceedings of the 5th NASA Langley Workshop on Formal Methods, June 2000

[69] Del Gobbo, D., Napolitano, M.R., "Issues in Fault Detectability for Dynamic Systems", Proceedings of the 2000 American Control Conference (ACC) Chicago, IL, June 2000

[70] Fravolini, M.L., Campa, G., Napolitano, M. R., "A Neural Network Based Tool for Aircraft SFDIA Modeling and Simulation", Proceedings of the IASTED Conference, Pittsburgh, PA, May 2001

[71] B.S. Kim, A.J. Calise, "Nonlinear Flight Control Using Neural Networks," Journal of Guidance, Control, and Dynamics, Vol.20, No.1, pp.26 33, 1997.

[72] Matlab User's Guide. Mathworks Inc. Natick, MA.

[73] Diamond D-MM32_AT Manual.

[74] Crossbow Technology Inc. IMU Manual.

[75] Novatel Inc. OEM4 Manual.

[76] C.Schumacher. "Adaptive Flight Control Using Dynamic Inversion and Neural Networks". AIAA-99-4086

[77] Joseph J.Totah. "Adaptive Flight Control and Online Learning"

[78] Rolf T. Rysdyk, A.J.Calise. "Fault Tolerant Flight Control via Adaptive Neural Network Augmentation” AIAA-98-4483 
[79] Y. Shin, M.D.Johnson. "Neural Network-Based Adaptive Control for Nonlinear Flight Regimes" AIAA Guidance, Navigation and Control Conference, August 2003.

[80] Seanor, B. "Flight Testing of a Remotely Piloted Vehicle for Aircraft Parameter Estimation Purposes" WVU Ph.D. Dissertation, 2002.

[81] Perhinschi M. G., Campa G., Napolitano M.R., Lando M., Massotti L., Fravolini M. L., "Modeling and Simulation of Failures for Primary Control Surfaces", AIAA2002-4786 AIAA Proceedings of the AIAA Modeling and Simulation Conference, Monterey, CA 2002

[82] Perhinschi M. G., Napolitano M.R., Campa G., Fravolini M. L., "Primary Control Surface Failure Detection and Identification Scheme", Proceedings of the AIAA Guidance, Navigation, and Control Conference, August 2003, Austin, Texas, AIAA-2003-5645

[83] A.J.Calise, S.Lee. "Direct Adaptive reconfigurable Cotnrol of a Tailless Fighter Aircraft" AIAA-98-4108

[84] U-Web Student Web Service. "Neural Network Toolbox - Backpropagation" http://www.uweb.ucsb.edu/ weibin/nnet/backpr5.html

[85] Wolfram Engineering. "Curve Fitting, Regression", http://www.efunda.com/math/leastsquares/leastsquares.cfm 